\title{
PINS Spectrum Identification Guide
}

\author{
A. J. Caffrey
}

March 2012

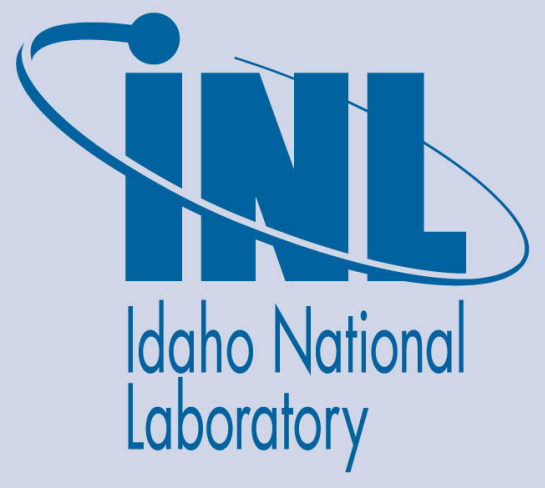

The INL is a U.S. Department of Energy National Laboratory operated by Battelle Energy Alliance 
INL/EXT-12-25401

\title{
PINS Spectrum Identification Guide
}

\author{
A. J. Caffrey
}

March 2012

\section{Idaho National Laboratory \\ Idaho Falls, Idaho 83415}

http://www.inl.gov

\author{
Prepared for the \\ U.S. Army \\ and for the
}

U.S. Department of Energy

Under DOE Idaho Operations Office

Contract DE-AC07-05ID14517 


\title{
PINS Spectrum Identification Guide
}

\author{
A.J. Caffrey \\ Idaho National Laboratory
}

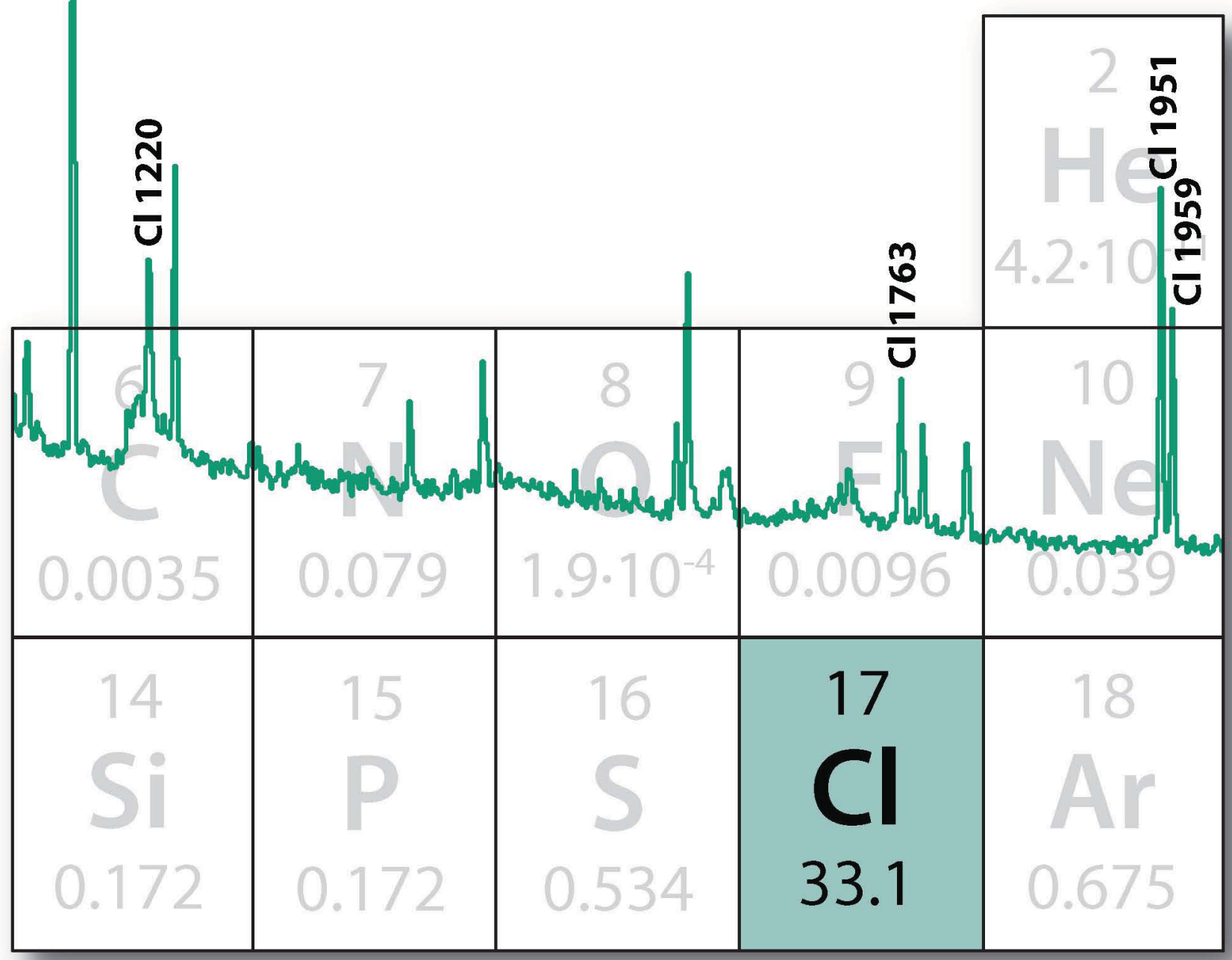




\section{DISCLAIMER}

This information was prepared as an account of work sponsored by an agency of the U.S. Government. Neither the U.S. Government nor any agency thereof, nor any of their employees, makes any warranty, expressed or implied, or assumes any legal liability or responsibility for the accuracy, completeness, or usefulness, of any information, apparatus, product, or process disclosed, or represents that its use would not infringe privately owned rights. References herein to any specific commercial product, process, or service by trade name, trade mark, manufacturer, or otherwise, does not necessarily constitute or imply its endorsement, recommendation, or favoring by the U.S. Government or any agency thereof. The views and opinions of authors expressed herein do not necessarily state or reflect those of the U.S. Government or any agency thereof. 


\title{
PINS Spectrum Identification Guide
}

\author{
A.J. Caffrey \\ Idaho National Laboratory \\ Idaho Falls, Idaho USA 83415 \\ March 2012 \\ revised, February 2013
}





\section{Preface}

The Portable Isotopic Neutron Spectroscopy — PINS, for short - system identifies the chemicals inside munitions and containers without opening them, a decided safety advantage if the fill chemical is a hazardous substance like a chemical warfare agent or an explosive. The U.S. military has used PINS for nearly two decades for treaty verification, counter-terrorism, and identification of range-recovered items. In the latter case, many rangerecovered munitions have lost their descriptive markings due to corrosion, especially those buried at the time of World War I.

PINS chemical identification is performed by a type of neutron activation analysis (NAA), known as prompt gamma-ray neutron activation analysis (PGNAA). As its name suggests, PGNAA is a neutron-in, gamma-ray-out analysis technique: neutrons from a small radioactive source or an electrical neutron generator irradiate the object under test, and interact with the nuclei of the chemical elements within the object, promoting some of those nuclei to an excited state. In turn, the nuclei de-excite promptly by emission of gamma rays. Many of the gamma rays escape the container or munition, to be measured by a gamma-ray spectrometer. Analysis of the resulting gamma-ray energyintensity pattern, or spectrum, identifies the chemical elements within the object under test.

The Guide is divided into two parts. The three chapters that constitute Part I cover the science and technology of PINS. Neutron activation analysis is the focus of Chapter 1. Chapter 2 explores PINS hardware, software, and related operational issues. Gamma-ray spectral analysis basics are introduced in Chapter 3.
The six chapters of Part II cover the identification of PINS spectra in detail. Like the PINS decision tree logic, these chapters are organized by chemical element: phosphorusbased chemicals, chlorine-based chemicals, etc. These descriptions of hazardous, toxic, and/or explosive chemicals are followed by a chapter on the identification of the inert chemicals, e.g. sand, used to fill practice munitions.

The Guide concludes with two appendices. The first, on hazardous industrial chemicals, could be another 100-page document in its own right, but it is limited to four examples of PINS identification. The second provides a table of gamma-ray energies relevant to PINS measurements."

The PINS Spectrum Identification Guide is intended as a reference for technical professionals responsible for the interpretation of PINS gamma-ray spectra. Even though PINS spectrum analysis algorithms have improved significantly over the years, there is still no substitute for expert review of PINS spectra, given the consequences of misidentifying munitions filled with chemical warfare agents or explosives.

The Guide presents the way PINS spectra are interpreted at Idaho National Laboratory. May it prove helpful to anyone who must stare at a computer display and ask, "What is this PINS spectrum trying to tell me?"

- Gus Caffrey

Idaho Falls, ID and Seabrook, NH

March 2012 


\section{Acknowledgments}

A t Idaho National Laboratory, the development of PINS has been a multidisciplinary effort since project inception in 1991. Mechanical engineer John Zabriskie designed the elegant but lightweight equipment stands, plus the shipping boxes for the gear. Nuclear engineers David Chichester and Woo Yoon have made important Monte Carlo design studies, and David helped incorporate an electrical neutron generator in the PINS3 system. Computer scientists Ann Egger, Steve Frickey, Ken Krebs, and Marie Putnam have tended the PINS data acquisition and data analysis software through numerous revisions and upgrades. Ken has also assisted with training and measurements in the field. Radiochemists John Baker, Jack Hartwell, and Cathy Riddle prepared simulant chemicals for research and training, and Jack helped measure PINS spectra, both in the lab and in the field. Chemist Catherine Crowder has recently taken over PINS simulant chemical production. Technical writer and graphic artist Amy Siedenstrang wrote and illustrated the PINS User's Manual, a perennial best-seller for a national laboratory report.

INL physicists Bob Gehrke, Brian Harlow, Gary McLaughlin, Ed Seabury, Kevin Watts, and Jayson Wharton have patiently carried out extensive development tests, and they helped make most of the critical design decisions. With Jerry Cole and Ken Krebs, they also helped record PINS data "behind the fence" with actual or suspect chemical weapons at numerous U.S. sites. INL statistician Larry Blackwood and INL theoretical physicist Clint Van Siclen have devised elegant and far-reaching upgrades to the PINS chemical-identification algorithm. Last and certainly not least, our retired colleague Reg Greenwood suggested neutron activation analysis for the nondestructive assay of chemical weapons, and he made major contributions to the design of the first PINS prototype.
Lt. Col. A.J. Kuehn of the On-Site Inspection Agency (now the Defense Threat Reduction Agency) and Leonard Rowe and Bill Brankowitz of the Office of the U.S. Army Project Manager for Non-Stockpile Chemical Materiel provided a fast education on the specialized lore of chemical warfare.

Since 1995, the ORTEC division of Ametek, Inc. has been INL's commercial partner for the PINS system. ORTEC's Benson Davis, Ron Keyser, Bob MacKenzie, T.J. Paulus, Tim Twomey, Dan Upp, and Doug Van Cleef have been especially helpful in the continued development of the instrument.

In preparing this Guide, Ed Seabury and Jayson Wharton helped round up gamma-ray spectra for the illustrations. Kris Burnham performed the elegant graphical layout. Ken Krebs wrote a special Igor macro to draw the spectral graphs. Ed and Ken also helped revise the gamma-ray energy table.

A special word of thanks is due my colleague Ann Egger. Besides maintaining the legacy Gauss nonlinear least-squares analysis code used in PINS spectral analyses, Ann drew and formatted the spectral graphs, compiled the index, and proofread mulitiple drafts of the text and illustrations with great care.

PINS research and development at Idaho National Laboratory is supported by the U.S. Army Project Manager for Non-Stockpile Chemical Materiel, under U.S. Department of Energy Field Office, Idaho contract number DE-AC07-05ID14517. 


\section{Table of Contents}

Preface

Acknowledgements

Glossary

Chapter 1: Neutron Activation Analysis . . . . . . . . . 1

Chapter 2: Field Neutron Activation Analysis with PINS . . . . . . . . . . . . . . . 11

Chapter 3: Gamma-Ray Spectrum Analysis Basics. . . . 17

Chapter 4: PINS Spectrum Identification Logic . . . . 25

Chapter 5: Phosphorus-Based Chemical Identification .29

P: White Phosphorus ................... 31

P, H: GB. . . . . . . . . . . . . . 33

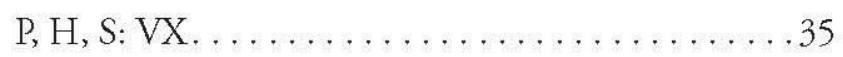

P, H, N, Cl: GA . . . . . . . . . . . . . . 37

Chapter 6: Arsenical Chemical Identification . . . . . . 39

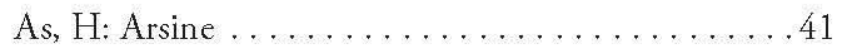

As, Cl: Arsenic Trichloride ............. 43

As, Cl, H: Blue Ring- $2 \ldots \ldots \ldots \ldots \ldots \ldots$

As, $\mathrm{Cl}, \mathrm{H}$ : Lewisite . . . . . . . . . . . . . . . . 47

As, Cl, H, S: Winterlöst . . . . . . . . . . . . 49

Chapter 7: Chlorine-Based Chemical Identification ...51

Cl: Phosgene. . . . . . . . . . . . . . . 53

$\mathrm{Cl}, \mathrm{H}: \mathrm{CNB} \ldots \ldots \ldots \ldots \ldots \ldots \ldots$

$\mathrm{Cl}, \mathrm{H}: \mathrm{CNS} \ldots \ldots \ldots \ldots \ldots \ldots \ldots \ldots \ldots \ldots$

Cl, Sn: KJ Smoke . . . . . . . . . . . . . . . . . . . 59

Cl, Ti: FM Smoke. . . . . . . . . . . . . . .61

Cl, Zn: HC Smoke. . . . . . . . . . . . . . 63

Cl, H, S: FS Smoke . . . . . . . . . . . . . 65

Cl, H, S: Sulfur Mustard. . . . . . . . . . . . . . 67

$\mathrm{Cl}, \mathrm{N}: \mathrm{CK} . \ldots \ldots \ldots \ldots \ldots \ldots \ldots \ldots$

Cl, N, H: Nitrogen Mustard-3 . . . . . . . . 71
Chapter 8: Bromine-Based Chemical Identification. . . .73

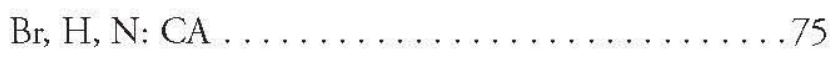

Chapter 9: Nitrogen-Based Explosives Identification . . .77 N, H: High explosives $\ldots \ldots \ldots \ldots \ldots \ldots . . .61$

Chapter 10: Practice-Fill Chemical Identification . . . . 83

B, H: Water/Anti-Freeze . . . . . . . . . . . . . . . 85

$\mathrm{Ca}, \mathrm{H}$, Si: Concrete . . . . . . . . . . . . 87

$\mathrm{Ca}, \mathrm{H}, \mathrm{S}$ : Plaster-of-Paris . . . . . . . . . . . . . 89

$\mathrm{Ca}, \mathrm{Cl}, \mathrm{H}$ : Calcium Decontamination Bleach. . . . .991

$\mathrm{Na}, \mathrm{Cl}, \mathrm{H}$ : Sodium Decontamination Bleach ......93

Si: Sand

Appendix A: Hazardous Industrial Chemical

Identification. . . . . . . . . . . . . . . . .97

$\mathrm{Ca}, \mathrm{Si}, \mathrm{H}$ : Acetylene . . . . . . . . . . . . . . . . 999

$\mathrm{Cl}$ : Chlorine . . . . . . . . . . . . . . . . 101

F, H: Hydrogen Fluoride. . . . . . . . . . . . 103

Hg: Mercury. . . . . . . . . . . . . . . . . 105

Appendix B: Gamma-Ray Energy Table . . . . . . . . 107

Index $\ldots \ldots \ldots \ldots \ldots \ldots \ldots \ldots \ldots \ldots \ldots \ldots \ldots \ldots$ 


\section{Glossary}

AKA also known as

barn $10^{-24}$ square centimeter

BR-2 blister agent; phenyldichloroarsine and diphenylchloroarsine mixture

$\mathrm{C}_{2} \mathrm{H}_{2}$ acetylene gas

CA bromobenzyl cyanide riot control agent

Cf-252 californium-252

CG phosgene choking agent

channel spectrum array element or histogram memory address

CK cyanogen chloride

$\mathrm{Cl}_{2}$ chlorine gas

$\mathrm{CN}$ riot control agent chloroacetophenone, also known as "Mace"

$\mathrm{CNB}$ riot control agent; chloroacetophenone, carbon tetrachloride, and benzene mixture

CNS riot control agent; chloroacetophenone, chloropicrin, and chloroform mixture

CWC Chemical Weapons Convention

DA diphenylchoroarsine vomiting agent

EOD explosive ordnance disposal

$\mathrm{eV}$ electron volt

$\mathrm{F}_{2}$ fluorine gas

FM titanium tetrachloride screening smoke

FS screening smoke; chlorosulfonic acid and sulfur trioxide mixture

FWHM full-width at half-maximum

GA nonpersistent nerve agent; tabun

Gaussian bell-shaped curve used to fit gamma-ray peaks by the PINS+ software

GB nonpersistent nerve agent; sarin

$\mathrm{H}$ sulfur mustard blister agent

$\mathrm{HC}$ hexachloroethane-based screening smoke

HD distilled sulfur mustard blister agent

HE high explosive

HF hydrogen fluoride gas
HN-3 nitrogen mustard-3 blister agent

HPGe high purity germanium

INL Idaho National Laboratory

Kelvin( $\mathrm{K})$ temperature above absolute zero; $0 \mathrm{~K}=-273^{\circ} \mathrm{C}=-460^{\circ} \mathrm{F}$

$\mathrm{keV}$ kilo-electron-volt; 1,000 electron volts

KJ stannic chloride screening smoke

$\mathrm{kV}$ kilovolt; 1,000 volts

L lewisite

MCA multichannel analyzer

$\mathrm{MeV}$ mega-electron-volt; 1,000 kilo-electron-volts mustard sulfur mustard blister agent

NATO North Atlantic Treatry Organization

NAA neutron activation analysis

$(\mathrm{n}, \alpha)$ neutron capture reaction producing an alpha particle

$(\mathrm{n}, \gamma)$ neutron capture reaction producing a gamma ray

$\left(\mathrm{n}, \mathrm{n}^{\prime} \gamma\right)$ inelastic neutron scattering reaction, producing a gamma ray

PD phenyldichloroarsine blister agent

PGNAA prompt gamma-ray neutron activation analysis

pile-up distortion of a spectrum at high counting rates

PINS Portable Isotopic Neutron Spectroscopy

PINS+ data acquisition and analysis computer program used with PINS systems

POP plaster-of-Paris

SA arsine blood agent; arsenic trihydride

$t^{*}$ test statistic for peak detection confidence level

VX persistent nerve agent; O-ethyl-S-(2dissopropylaminethyl) methyl phosphonate

W/AF water/antifreeze mixture

WL winter mustard; sulfur mustard and phenyldichloroarsine mixture

WP white phosphorus screening smoke wt- $\%$ elemental concentration in $\%$, by weight 


\section{Chapter 1 \\ Neutron Activation Analysis}

A bsent electrical charge, neutrons penetrate the "Coulomb barrier" surrounding an atomic nucleus with ease. In a collision with a nucleus, a neutron can excite it or transform it, and in either instance the target nucleus will usually emit radiation. If the radiation is measured, the target nucleus often can be identified, as neutron-induced radiations are often characteristic of the emitting isotope. For example, only the isotope nitrogen-15 emits a 10.8-MeV gamma ray.[1] Since isotopes correspond to specific chemical elements, one can perform elemental chemical analyses with neutrons, nondestructively.

This chapter describes neutron activation analysis, neutron interactions in materials, and the interactions of neutroninduced gamma rays.

\section{Delayed vs. Prompt Neutron Activation Analysis}

Neutron activation is commonly performed inside nuclear reactors. The object under test can be irradiated with fluxes exceeding $10^{10}$ neutrons $/ \mathrm{cm}^{2}$-sec, and impurities detected at parts-per-million levels. After the irradiation period, the object is moved to be counted by a detector in a shielded location, far away from the reactor. Because the measured radiation is delayed by its beta-decay half-life, this method is called delayed neutron activation analysis.

Of course, operators of nuclear reactors do not welcome the introduction of munitions to their facilities, especially suspect chemical warfare munitions. Another nondestructive analysis technique, prompt neutron activation analysis permits in situ examination of munitions. In the prompt method, the neutron-induced radiation is measured concurrently with neutron bombardment. PINS measures the gamma rays produced during neutron irradiation of an object, and this technique is called prompt gamma-ray neutron activation analysis, or PGNAA for short.

\section{Neutron Production}

For portability and safety, PINS systems operate with a much lower neutron flux than nuclear reactors, about $10^{3}$ neutrons $/ \mathrm{cm}^{2}$-sec. PINS neutrons are emitted by either radioisotopes or fusion reactions in electrical neutron generators. The advantages and shortcomings of each type of neutron source are discussed in the next two sections.

\section{Radioisotopic Neutron Sources}

The man-made elements beyond uranium in the periodic table grow increasingly unstable as a function of atomic weight. Most of these elements decay via alpha-particle emission, but a few also decay via spontaneous fission.

The isotope californium- 252 decays about $10 \%$ of the time by spontaneous fission, and each fission produces four neutrons, on average. Because of its 2.65-year half-life, californium-252 has a high specific activity: 1 microgram of this isotope emits 2 million neutrons per second. Securely packaged in a stainless-steel capsule, californium- 252 is a convenient neutron source for a variety of nondestructive evaluation instruments, including PINS.[1]

Other radioisotopic sources use an alpha-emitting substance mixed with beryllium powder. The most common of these sources uses americium-241 as the alpha-particle emitter, but plutonium-239 and radium-226 are also used. These sources produce neutrons from an " $(\alpha, n)$ " reaction:

$$
\alpha+{ }^{9} \mathrm{Be} \rightarrow{ }^{12} \mathrm{C}+\mathrm{n} \text {. }
$$


Californium-252 (Cf-252) produces a Maxwellian neutron energy spectrum of the form

$$
\frac{d N}{d E}=N_{0} \sqrt{E} \exp (\square E / T)
$$

where $\mathrm{E}$ is the neutron energy and $\mathrm{T}$, the most frequent neutron energy, is the temperature of the spectrum.[2] The californium-252 neutron energy spectrum is shown in Figure 1-1.

\section{Neutron Generators}

Some future PINS systems will employ electrical neutron generators. These devices accelerate a hydrogen isotope into a hydrogen isotope-implanted target, causing a fusion reaction. If the collision is between two deuterium $\left({ }^{2} \mathrm{H}\right)$ nuclei, the $D D$ fusion reaction that produces neutrons is

$$
{ }^{2} \mathrm{H}+{ }^{2} \mathrm{H} \rightarrow{ }^{3} \mathrm{He}+\mathrm{n},
$$

and the outgoing neutron energy is $2.5 \mathrm{MeV}$. If the collision

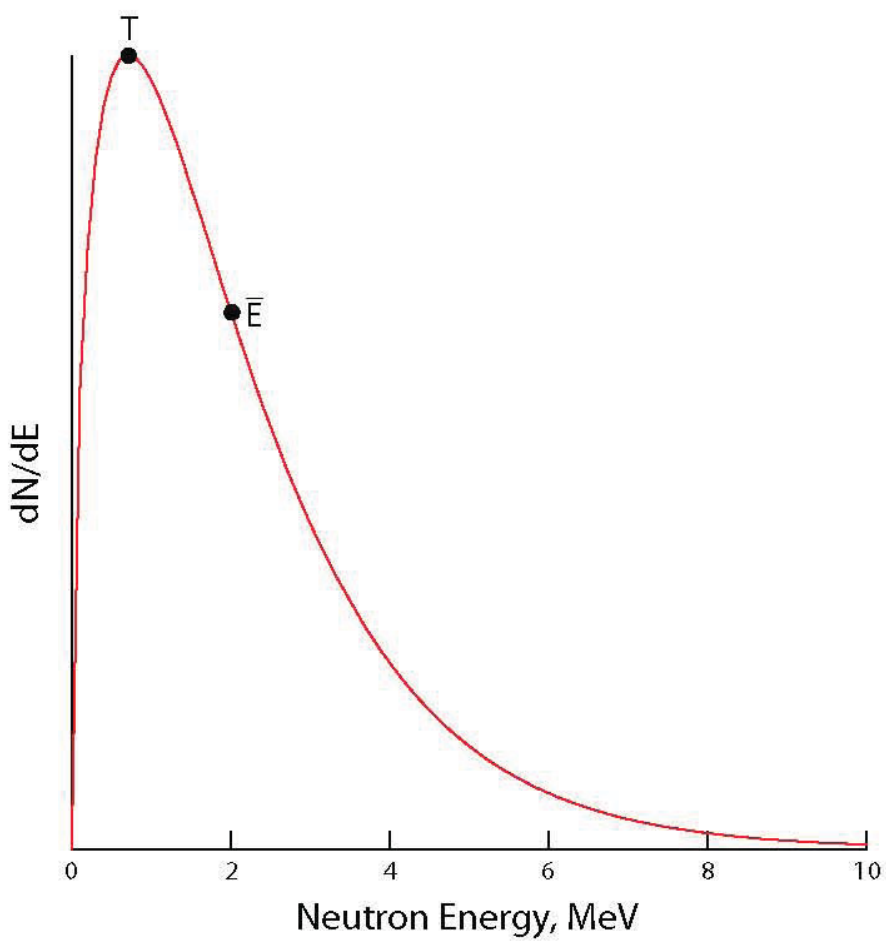

Figure 1-1: Cf-252 neutron energy spectrum. The spectrum temperature $T=1.42 \mathrm{MeV}$, and the mean energy $\bar{E}=2.13 \mathrm{MeV}$. involves a deuteron $\left({ }^{2} \mathrm{H}\right)$ and a triton $\left({ }^{3} \mathrm{H}\right)$, the $D T$ fusion reaction

$$
{ }^{2} \mathrm{H}+{ }^{3} \mathrm{H} \rightarrow{ }^{4} \mathrm{He}+\mathrm{n}
$$

produces a $14.1-\mathrm{MeV}$ neutron. The neutron energy spectra of a californium-252 radioisotopic source and two types of neutron generators are compared in Figure 1-2.

\section{Neutron Interactions}

Neutrons rarely interact with atomic electrons, and hence neutron-based chemical assay methods provide no information on molecular bonds. As noted above, neutrons do interact with atomic nuclei, and they do so via elastic scattering, inelastic scattering, and capture reactions.

\section{Elastic Scattering}

Elastic scattering is a process of bouncing a neutron off an atomic nucleus; the target nucleus is neither excited nor transformed. The net effects of elastic scattering are neutron redirection and neutron energy loss.

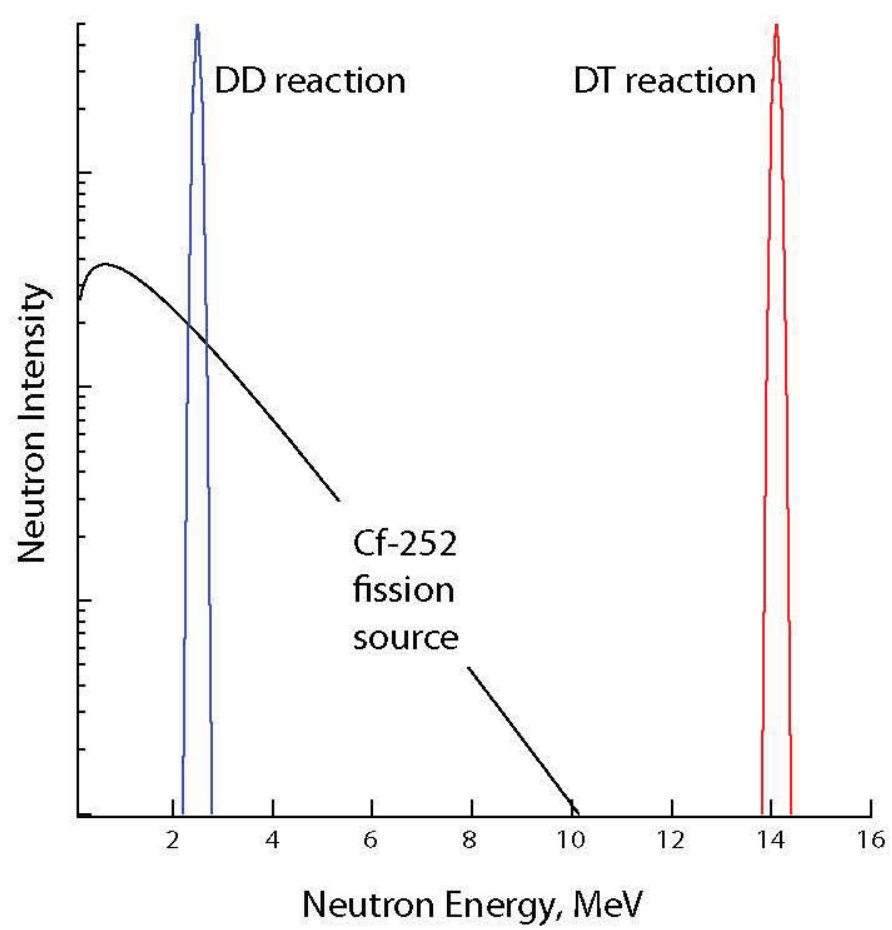

Figure 1-2: Comparison of neutron energy spectra from $C f-252, D D$, and DT neutron generators. 
Let the incident neutron energy be denoted $E_{n}$, the target nucleus atomic weight $A$, and the recoil energy of the target nucleus be $\mathrm{E}_{\mathrm{R}}$. As Knoll shows in his text [3], the maximum fractional energy transfer in neutron elastic scattering is

$$
\frac{E_{R}}{E_{n}}=\frac{4 A}{(1+A)^{2}} .
$$

The maximum energy transfer falls dramatically with increasing atomic weight, as shown in Figure 1-3.

Neutrons lose at most a few percent of their kinetic energy when scattering from heavy nuclei, say lead or tungsten. The neutron is simply redirected, like a rubber ball striking a concrete wall.

Neutron scattering kinematics are quite different for light nuclei. When the target nucleus is a proton, the atomic weight $\mathrm{A}=1$, and the energy loss can be total. On average, a neutron loses half of its kinetic energy in each collision with a proton, [4] the nucleus of ordinary hydrogen-1 $\left({ }^{1} \mathrm{H}\right)$. Ten elastic collisions with protons will reduce a neutron's kinetic energy by a factor of $2^{10}=1,024$, and twenty collisions will reduce the neutron's kinetic energy by a factor of $2^{20} \approx 1$ million. The slowing of neutrons by elastic scattering is called moderation, and it is an important parameter in nuclear reactor design. The abundance of hydrogen in water makes it a good neutron moderator. In PINS instruments, hydrogen-bearing polyethylene is used to moderate neutrons.

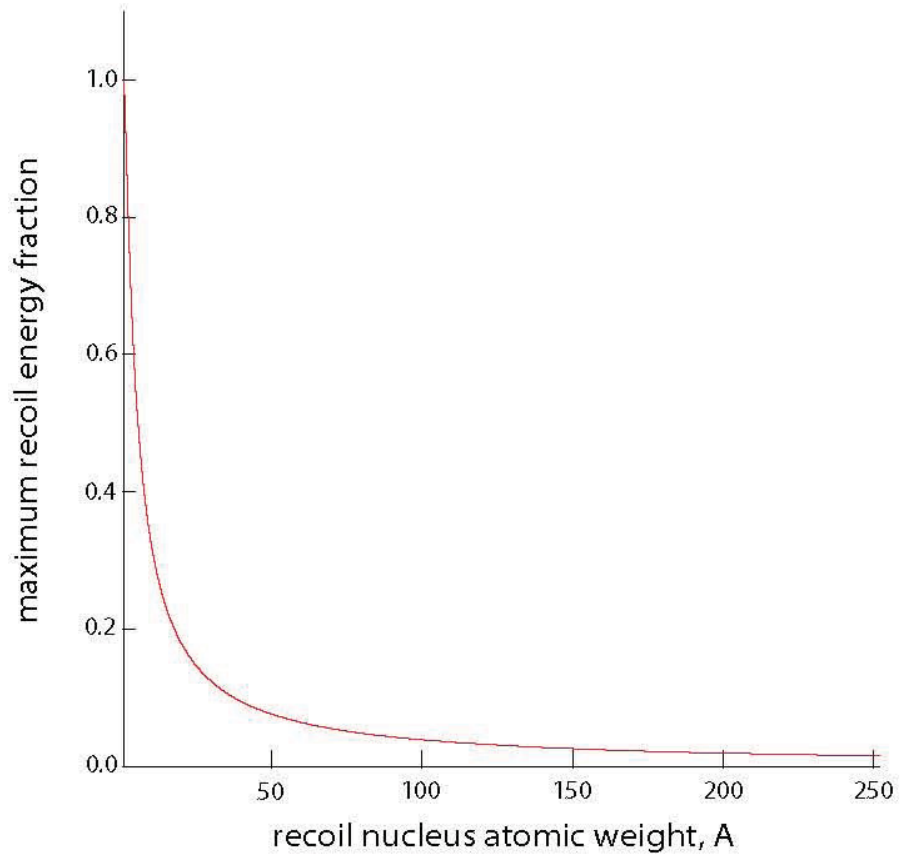

Figure 1-3: Maximum energy transfer in elastic scattering vs. recoil nucleus atomic weight.

\section{Inelastic Scattering}

Inelastic scattering is also a process of bouncing a neutron off a nucleus, but in this case the struck nucleus is promoted to an excited state. Usually the nucleus quickly de-excites to its ground state by gamma-ray emission. As an example, neutron inelastic scattering on silicon proceeds by this series of events:

$$
\mathrm{n}+{ }^{28} \mathrm{Si} \rightarrow \mathrm{n}^{\prime}+{ }^{28} \mathrm{Si}^{*} \rightarrow{ }^{28} \mathrm{Si} \text { (ground state) }+\gamma \cdot
$$

Here the ${ }^{*}$ character indicates an excited state of the silicon nucleus. At the end of the process, when the silicon nucleus has emitted a $1779-\mathrm{keV}$ gamma ray and returned to its ground state, it is essentially unchanged. The neutron loses energy and is redirected. Inelastic scattering from silicon is energetically forbidden unless the initial neutron energy is at least $1779 \mathrm{keV}$. Hence low-energy neutrons cannot scatter inelastically; they can only interact with nuclei by elastic scattering or neutron capture.

The first excited states of carbon-12 and oxygen-16 nuclei are at 4.4 and $6.1 \mathrm{MeV}$ respectively. The fraction of neutrons exceeding $4 \mathrm{MeV}$ emitted by $\mathrm{Cf}-252$ is too low to effectively excite either nucleus via inelastic scattering.

The inelastic scattering gamma-ray table of Demidov et al. covers most of the periodic table. [5]

\section{Neutron Capture}

Neutron capture is a process where a neutron binds to an atomic nucleus and transforms it, increasing its atomic weight by 1 . The transformed nucleus will be in a highly excited state due to the neutron binding energy, typically several $\mathrm{MeV}$ or more. The simplest neutron capture reaction is with hydrogen:

$$
\mathrm{n}+{ }^{1} \mathrm{H} \rightarrow{ }^{2} \mathrm{H}+\gamma
$$

In this reaction the neutron $(\mathrm{n})$ and the proton $\left({ }^{1} \mathrm{H}\right)$ bind to form a deuteron $\left({ }^{2} \mathrm{H}\right)$. The deuteron's binding energy is released in the form of a 2.2-MeV gamma ray. [6] Detailed tables of capture gamma rays for most of the elements in the periodic table are available. [7]

Nuclear fission is a special type of neutron capture. In fission, the added neutron binding energy is sufficient to cause an unstable isotope like uranium-235 to break apart or fission. 


\section{Neutron Cross Sections}

The likelihood of a subatomic particle interacting with a nucleus is measured by the reaction cross section. Imagine a thin circular plate of a substance of atomic weight $A$ and mass $\mathrm{m}$, in grams, at a distance $\mathrm{d} \mathrm{cm}$. from an isotropic neutron source emitting q neutrons per second. Using the inverse-square law, the neutron flux at the plate will be

$$
\phi=q /\left(4 \pi d^{2}\right) .
$$

Flux is usually expressed in neutrons per $\mathrm{cm}^{2}$-sec. The neutron reaction rate in the plate is

$$
\mathrm{R}=\phi(\mathrm{m} / \mathrm{A}) \mathrm{N}_{0} \sigma,
$$

and it has the dimension of reactions per second, where $\mathrm{N}_{0}$ is Avogadro's number and $\sigma$ is the reaction cross section.

Since the ratio m/A is dimensionless, and so is Avogadro's number, the dimension of $\sigma$ must be area, to cancel the area $4 \pi \mathrm{d}^{2}$ in the denominator of the neutron flux $\phi$. Because atomic nuclei have extremely small areas in metric units, cross sections are usually stated in barns, where

1 barn $\equiv 10^{-24} \mathrm{~cm}^{2}$.

Some exceptionally stable nuclei have "closed shells", akin to the closed electron shells of the noble gases, and these nuclei have small neutron capture cross sections. Notably, carbon- 12 and oxygen- 16 cannot be identified by capture gamma rays using a PINS Cf-252 neutron source.

\section{Cross Section Energy Dependence}

The neutron capture cross section for the element silver is shown in Figure 1-4.[8] The cross section is maximal at low energies, where a neutron is in thermal equilibrium with its surroundings; at room temperature thermal energy corresponds to $1 / 40 \mathrm{eV}$. The cross section falls with increasing neutron energy, until the "resonance region", and then above $5 \mathrm{keV}$ it falls monotonically.

This type of energy dependence is typical of most nuclei, even fissionable nuclei, and it explains why neutrons are moderated in nuclear reactors: to take advantage of the larger fission cross sections of neutrons at thermal energies.

\section{Cross Section Variation by Chemical Element}

The thermal energy neutron cross sections of the elements are shown in Figure 1-4.[9] Note that these cross sections vary by as much as six orders of magnitude. Importantly for PINS, the chlorine capture cross section is 33 barns, while for nitrogen it is only 0.08 barns.

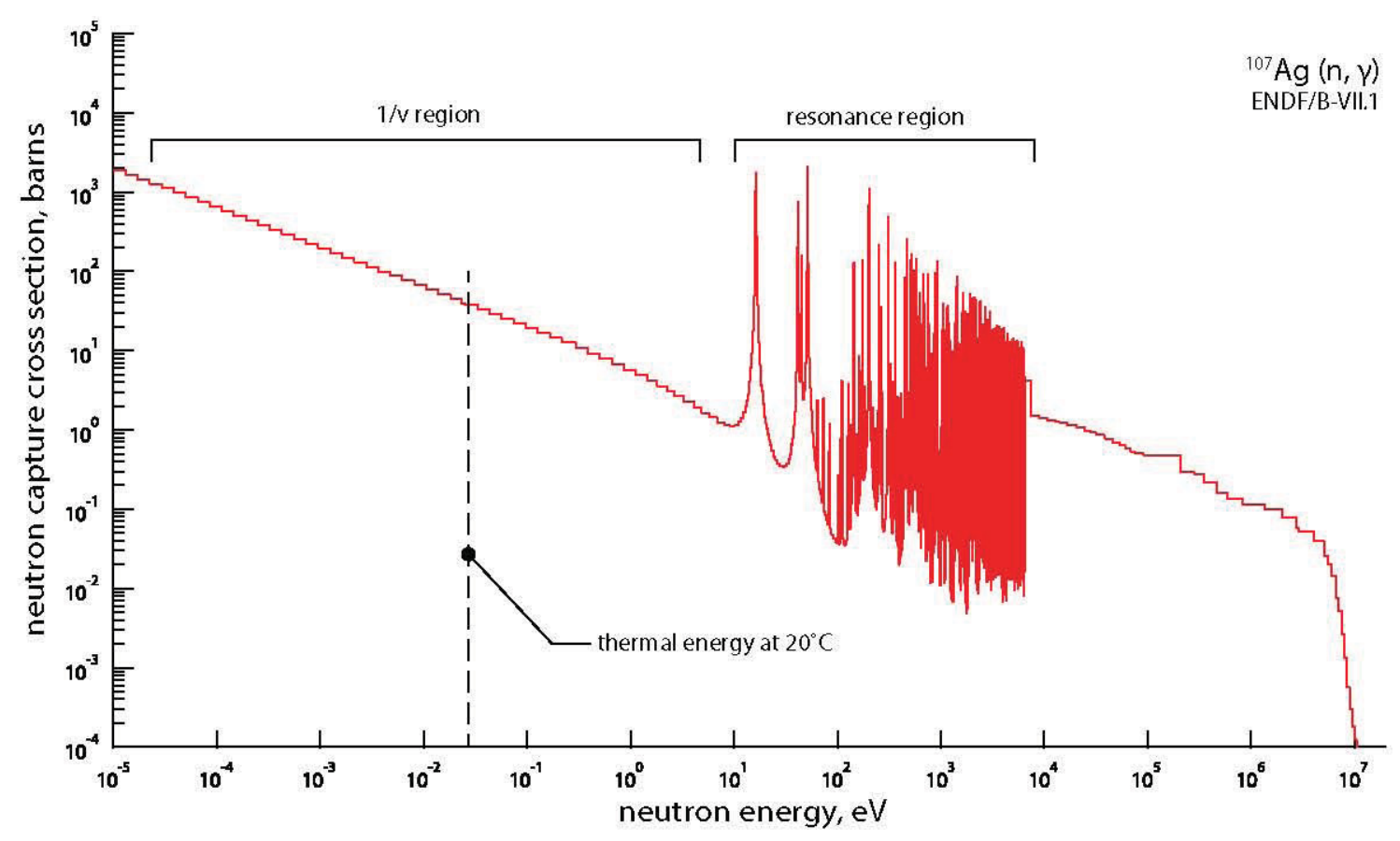

Figure 1-4: Neutron capture cross section vs. energy for ${ }^{107} \mathrm{Ag}$. 


\section{Neutron Transmission through Materials}

Atoms, while quite small - about $10^{-8} \mathrm{~cm}$ in diameter-are a factor of 10,000 larger than atomic nuclei. The nucleus is roughly $10^{-12} \mathrm{~cm}$ in size. An atom's size is really the size of its electron cloud. Since neutrons rarely interact with electrons, they pass through matter easily, and their path is interrupted only when the much smaller nucleus is encountered.

The best neutron shielding materials contain light nuclei, such as hydrogen and carbon. As we've seen, the heavy elements redirect neutrons without slowing them. Light materials do slow neutrons, and if a hydrogenous moderator like polyethylene, water, or wax is mixed with a substance having a large capture cross section for slow neutrons, a so-called poison, neutrons can be shielded quite effectively. Neutron poisons include boron, cadmium, and gadolinium.

cross section key: $\sigma_{\mathrm{a}}<10^{-2}$

$$
10^{-2}<\sigma_{a}<1
$$
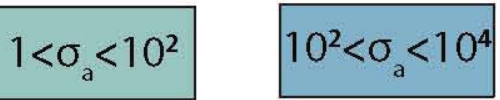

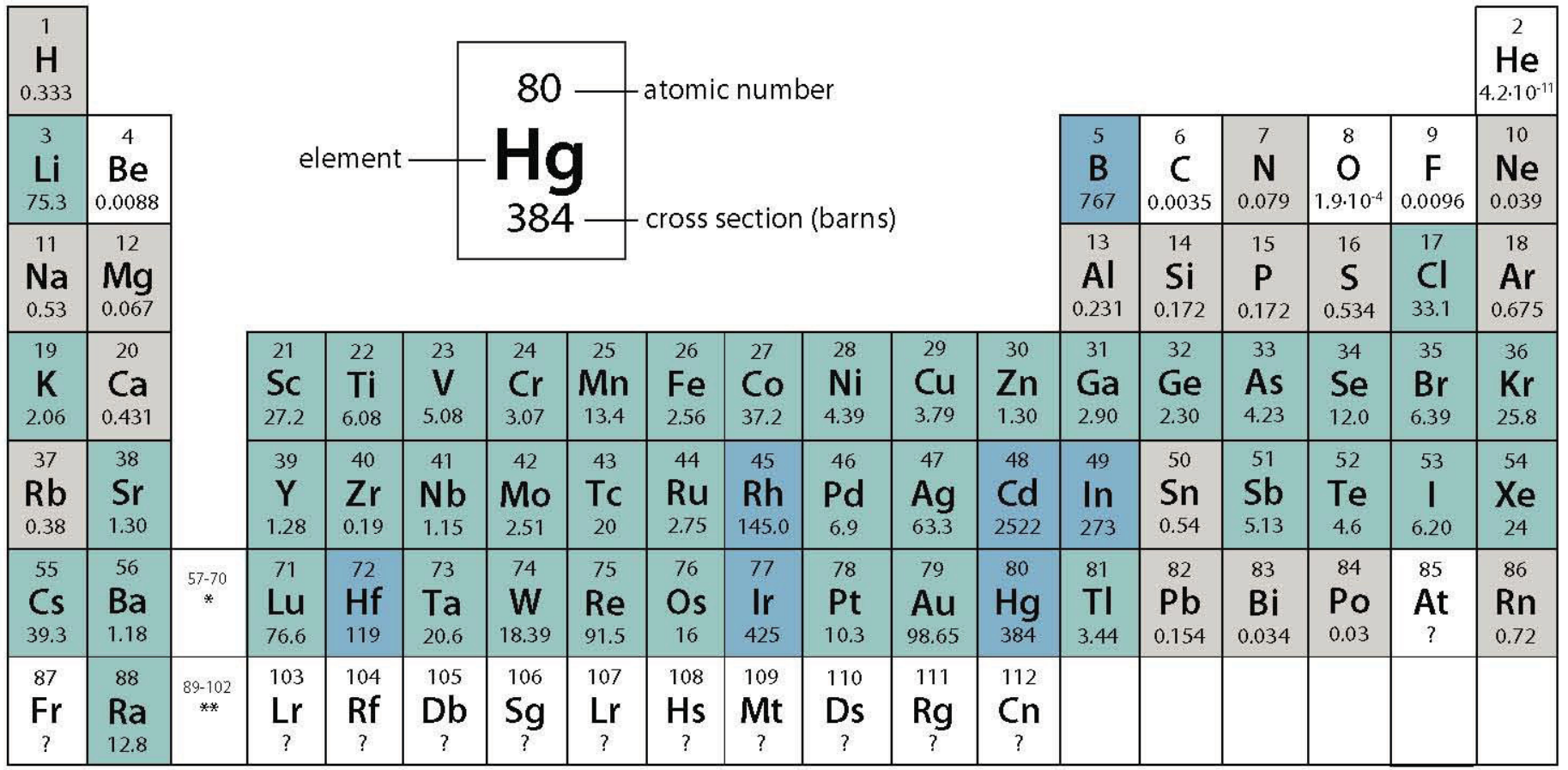

\begin{tabular}{|c|c|c|c|c|c|c|c|c|c|c|c|c|c|c|}
\hline & $\begin{array}{l}57 \\
\text { La } \\
9.08\end{array}$ & $\stackrel{58}{\mathrm{Ce}}$ & $\begin{array}{l}59 \\
\mathrm{Pr}\end{array}$ & $\begin{array}{l}60 \\
\mathrm{Nd}\end{array}$ & $\begin{array}{c}61 \\
\mathrm{Pm}\end{array}$ & $\mathrm{Sm}_{5621}^{62}$ & $\begin{array}{l}63 \\
\mathrm{Eu}\end{array}$ & $\stackrel{64}{\mathrm{Gd}}$ & 65 & 66 & 67 & $\begin{array}{l}68 \\
\mathrm{Er}\end{array}$ & & \\
\hline & $\begin{array}{c}89 \\
\mathrm{AC} \\
890\end{array}$ & $\begin{array}{l}\text { Th } \\
7.35\end{array}$ & $\begin{array}{c}91 \\
\mathrm{~Pa} \\
200.6\end{array}$ & $\underset{3.374}{\mathbf{U}}$ & 180 & $\begin{array}{c}94 \\
\mathbf{P u} \\
1017.3\end{array}$ & $\underset{587}{\mathrm{Am}}$ & $\mathrm{Cm}_{79}$ & 710 & $\begin{array}{c}\text { Cf } \\
2900\end{array}$ & 160 & & $?$ & \\
\hline
\end{tabular}

Figure 1-5: Thermal neutron capture cross sections of the elements. 


\section{Gamma-Ray Interactions in Matter}

Gamma rays, since they are electrically neutral, are also a very penetrating form of radiation. Gamma rays interact with materials by the three processes described below. The energy dependencies of these processes are shown in Figure 1-6.[10]

\section{Photoelectric effect}

A gamma-ray can be totally absorbed by an atom. To conserve energy, the gamma-ray energy is given to an atomic electron, and the electron is ejected from the atom. The photoelectric effect produces the photopeaks ("peaks", for short) in gamma-ray spectra, like the three natural background radioisotopic peaks shown in Figure 1-7.

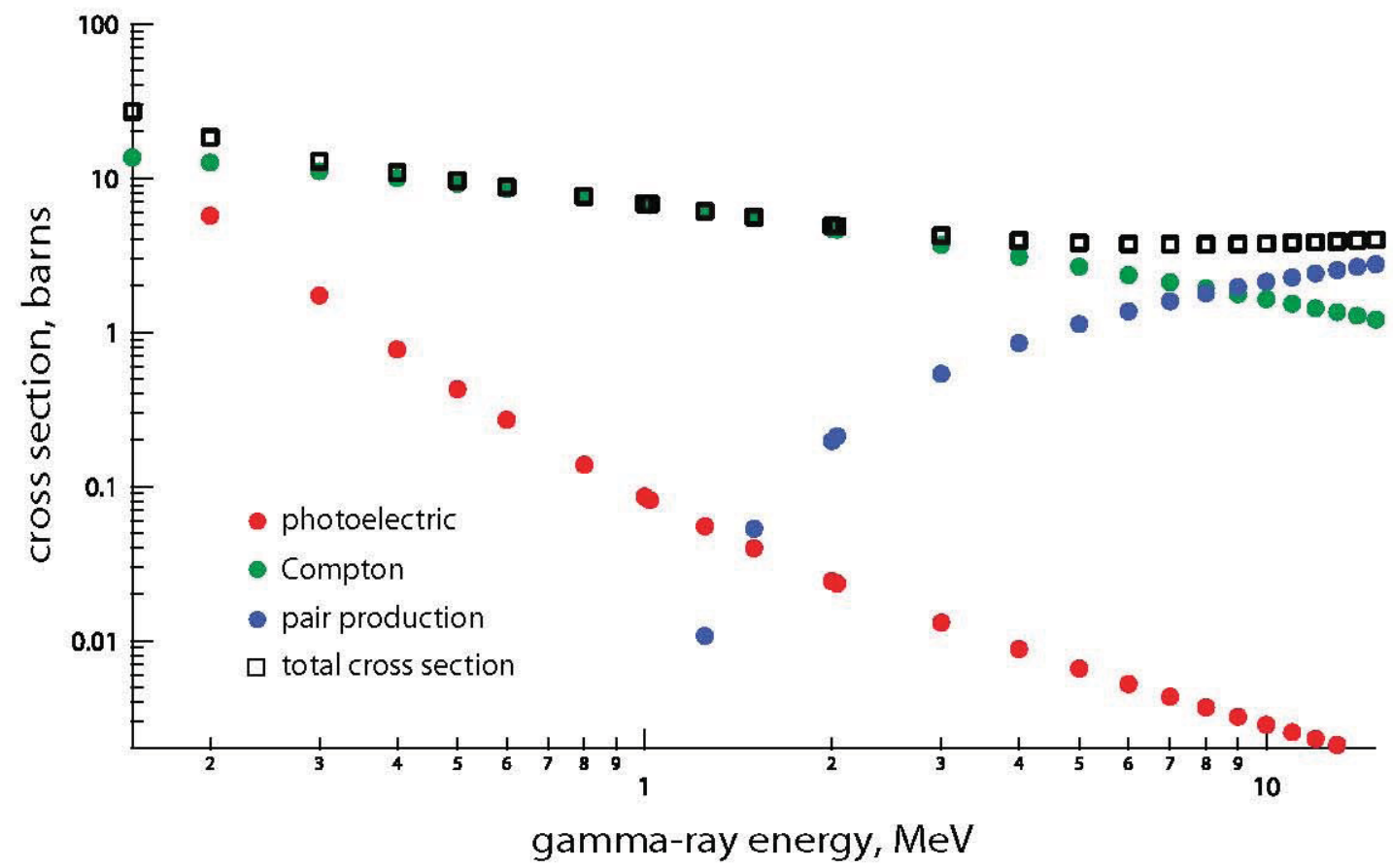

Figure 1-6: Gamma-ray interaction cross sections vs. energy.

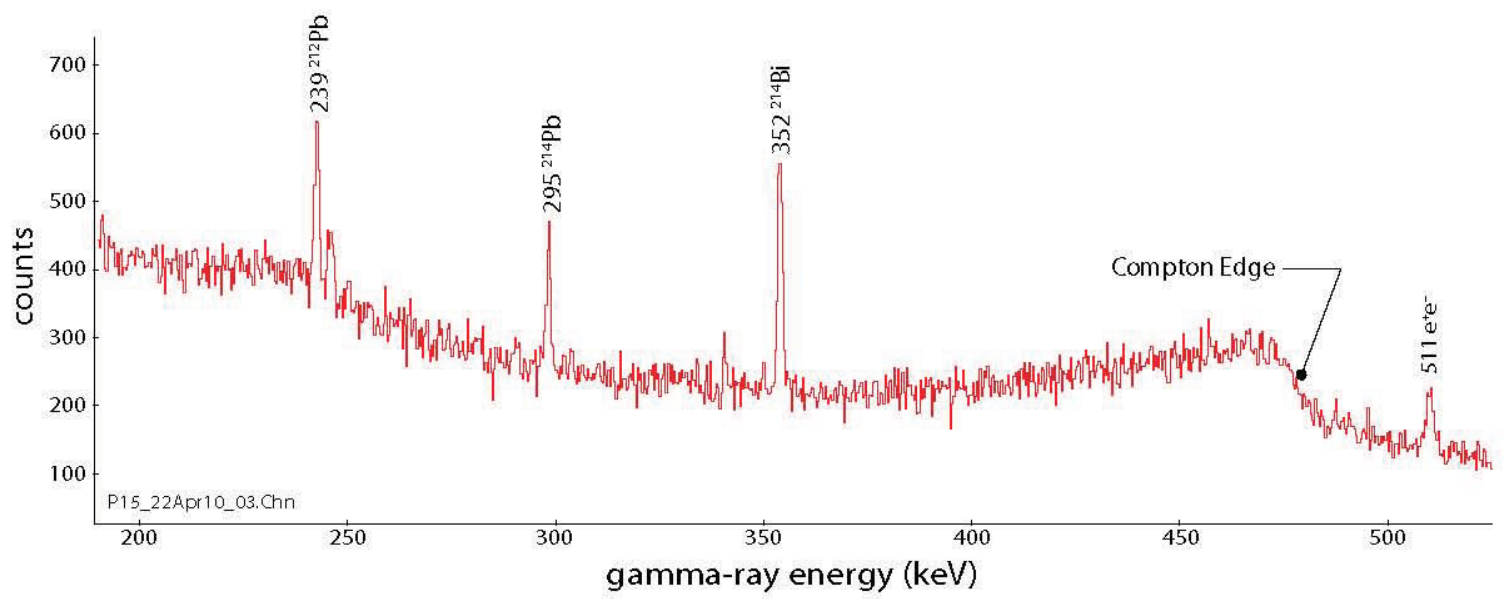

Figure 1-7: Gamma-ray photopeaks and the Compton continuum. 


\section{Compton effect}

Compton scattering transfers part of the gamma-ray energy to an electron. The scattered gamma-ray energy is lower, as required by energy and momentum conservation. As depicted in Figure 1-8, the scattered gamma-ray photon can escape the detector, producing an event where less than the full energy of the incident gamma ray is measured by the detector. In effect, some of the gamma ray energy has leaked out of the spectrometer.

The amount of energy transferred to the electron is dependent on the angle between the incident and scattered gamma rays. Because of the variable energy transferred to the electron, Compton scattering produces not a peak, but a continuum in a gamma-ray spectrum, also shown in Figure 1-7. The abrupt change in the continuum at the right of the figure is known as a Compton edge, and it corresponds to the maximum transfer of energy to the Compton electron, when the scattering angle is $180^{\circ}$.

\section{Pair production}

Pair production is the creation of an electron and its antiparticle, the positron, as a gamma ray passes near an atomic nucleus, as shown in Figure 1-9. The gamma ray is converted into an electron-positron pair. Pair production is forbidden by energy conservation unless the gamma-ray energy exceeds $1.022 \mathrm{MeV}$, or twice the electron rest-mass energy.

Also depicted in Figure 1-9, the positron then encounters another electron, and these antiparticles mutually annihilate, producing two $511-\mathrm{keV}$ gamma rays, corresponding to the two electron rest masses that were converted into energy. One of the annihilation gamma rays escapes the detector creating a single-escape event where the spectrometer measures the incident energy $\mathrm{E}_{\text {in }}-511 \mathrm{keV}$. Counts from single-escape events produce a single-escape peak in the gamma-ray spectrum.

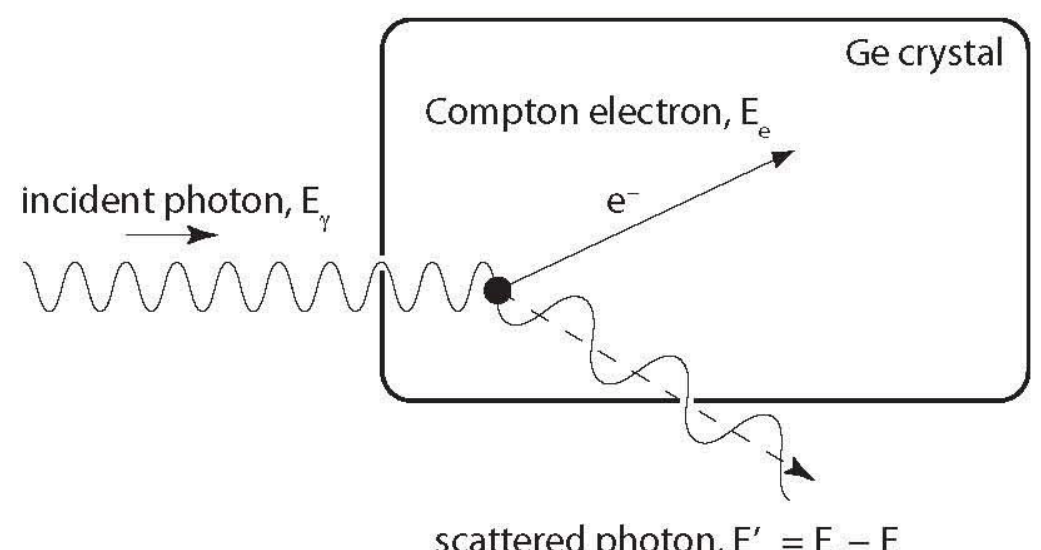

Figure 1-8: Schematic Compton scattering event.

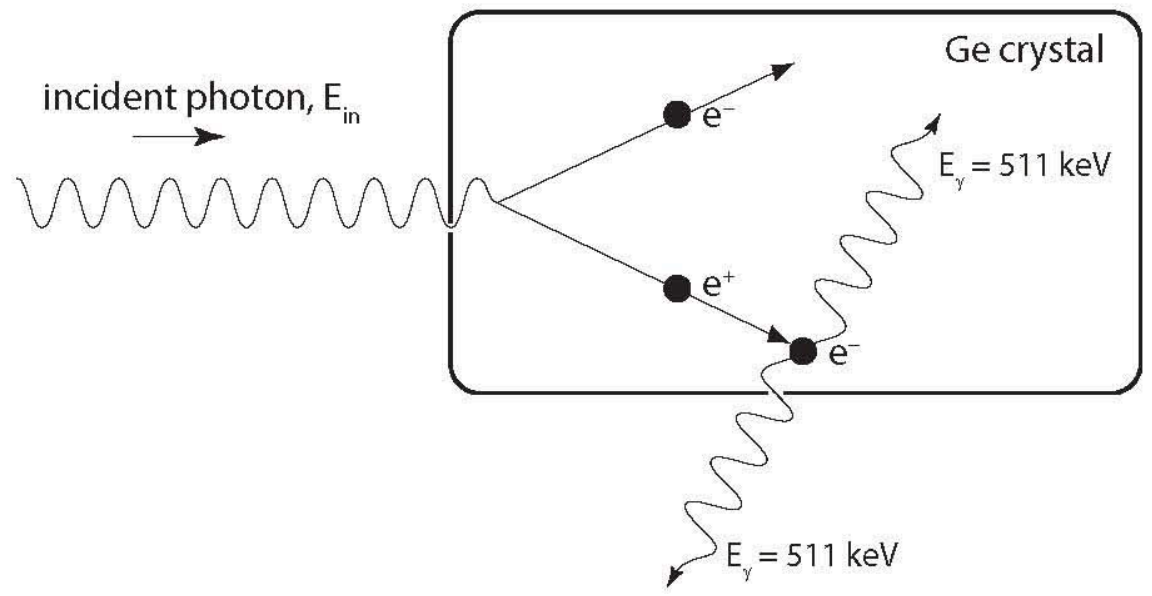

Figure 1-9: Pair production, followed by positron annihilation in the detector crystal.

One of the 511-keV annihilation gamma rays escapes the detector, causing a single-escape event. 
Double-escape events are also possible, where both annihilation gamma rays leave the detector crystal without interacting, and these events produce peaks at $\mathrm{E}_{\text {in }}-$ $1022 \mathrm{keV}$. Sometimes both annihilation gamma rays are contained in the crystal, and the spectrometer records a full energy event, placing a count in the peak at $\mathrm{E}_{\text {in }}$. Figure 1-10 displays the full-energy, single-escape, and double-escape peaks for a pair of iron gamma rays near $6 \mathrm{MeV}$.

\section{Gamma-ray transmission through materials}

Gamma rays interact primarily with atomic electrons, and the more electrons an atom has, the more likely gamma rays will interact with the atom. In fact, the photoelectric effect cross section for gamma rays scales as an absorber material's number of electrons $Z$ raised to the fourth power. Accordingly, low-Z chemical elements, that is, those at the beginning of the periodic table like carbon, are poor shielding for gamma rays. The high-Z, high-density metals like bismuth, lead, and tungsten are much better choices for gamma-ray shielding.

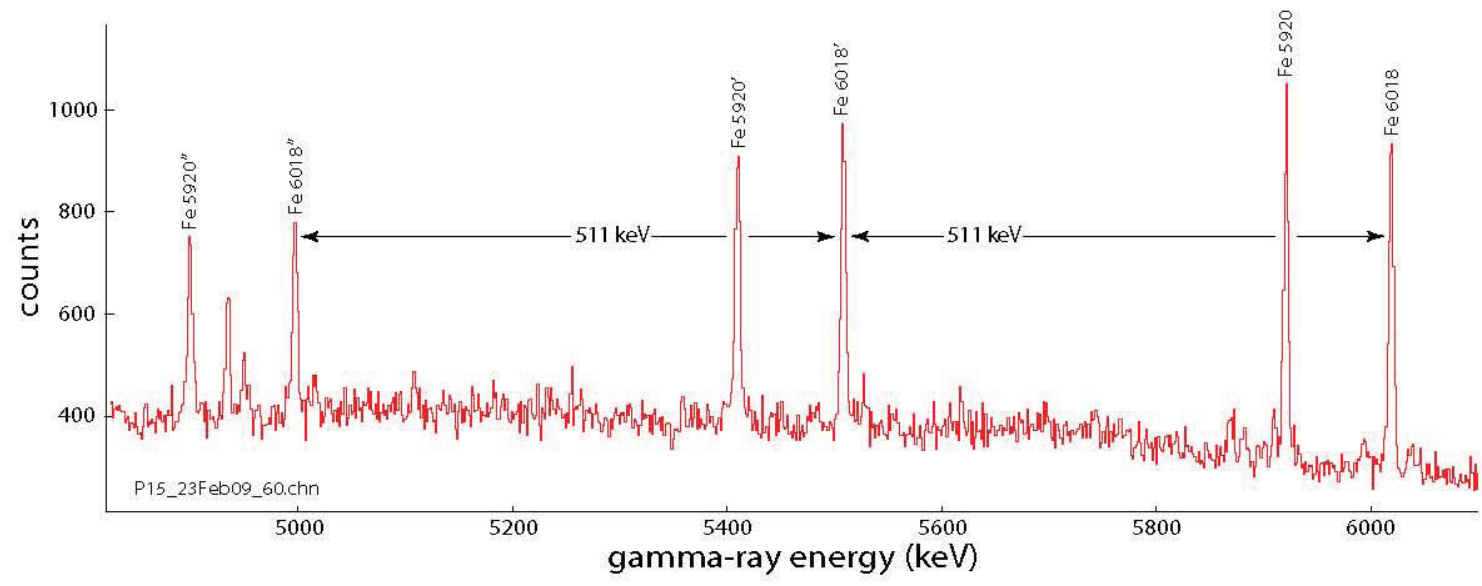

Figure 1-10: Two sets of iron fullenergy, single-escape, and double-escape peaks. Note the spacing between each set of peaks is equal to the electron rest-mass energy, $511 \mathrm{keV}$. Also note that single-escape and double-escape peaks are labeled with single (") and double (") prime characters, respectively. 


\section{References}

1. R.C. Martin, J.B. Knauer, and P.A. Balo, "Production, Distribution, and Applications of Californium-252

Neutron Sources", Proceedings of the 4th Topical Meeting on Industrial Radiation and Radioisotope Measurement Applications (IRRMA '99), Raleigh, North Carolina, October 1999. This paper is available on the web at www.ornl.gov/-webworks/cpr/pres/102606.pdf

2. J. Grundl and C.M. Eisenhauer, "Fission Spectrum Neutrons for Cross Section Validation and Neutron Flux Transfer", Proceedings of the Conference on Nuclear Cross Sections and Technology, National Bureau of Standards special publication NBS-425 (1975) pages 250-253. A. Smith, P. Guenther, R. Sjoblom, Nuclear Instruments and Methods 140 (1977) pages 397-400.

3. G.F.K noll, Radiation Detection and Measurement, Third Edition (New York: John Wiley \& Sons, 2000) pages 553-554.

4. Knoll, page 555.

5. A.M. Demidov, et al., Atlas of Gamma-Ray Spectra from Inelastic Scattering of Reactor Fast Neutrons (Moscow: Atomizdat, 1978)

6. The binding energy of the deuteron is an important physical constant. It is used to measure the strength of the attractive force between nucleons, and also to determine the neutron rest mass. For these reasons, Reg Greenwood of Idaho National Laboratory and Bob Chrien of Brookhaven National Laboratory measured this gamma-ray energy to 8-ppm accuracy: 2223.247 $\pm 0.017 \mathrm{keV}$. R.C. Greenwood and R.E. Chrien, "Neutron Mass: Measurement of the ${ }^{1} \mathrm{H}(\mathrm{n}, \gamma)^{2} \mathrm{H} \gamma$ ray and the revised values for selected neutron binding energies," Physical Review C21 (1980) pages 498-502.
7. M.A. Loan, R.A. Leavitt, and D.A. Harrison, "Prompt Gamma Rays from Thermal-Neutron Capture," Atomic Data and Nuclear Data Tables 26 (1981) page 528. G.L. Molnar, Handbook of Prompt Gamma Activation Analysis (Dordrecht, The Netherlands: Kluwer Academic Publishers, 2004). National Nuclear Data Center, Brookhaven National Laboratory "CapGam" website: www.nndc.bnl.gov/capgam/

8. Evaluated Nuclear Data File/B-VII.1 (December, 2011), National Nuclear Data Center, Brookhaven National Laboratory, available at this web site: http://www.nndc.bnl.gov/exfor/endf00.jsp

9. International Atomic Energy Agency Prompt Gamma-Ray Neutron Activation Analysis website: www-nds.iaea.org/pgaa/pgaa7/index.html

10. National Institute of Standards and Technology XCOM Photon Cross Sections Database website: www.nist.gov/pml/data/xcom/index.cfm 
10 PINS Spectrum Identification Guide 


\section{Chapter 2 Field Neutron \\ Activation Analysis with PINS}

A $s$ a portable neutron activation analysis system for field use, PINS employs reasonably compact equipment in a configuration that can be assembled quickly and in a reproducible geometry. The software for control, acquisition, and analysis is highly automated to allow users to operate PINS with confidence and ease.

\section{PINS Equipment}

The PINS equipment set includes a neutron source, the gamma-ray spectrometer and its readout electronics, the moderator/shadow-shield assembly, and the equipment stand. A PINS equipment set plus its three footlocker-sized shipping containers are shown in Figure 2-1.

\section{Californium-252 Neutron Source}

The construction of the californium-252 (Cf-252) source is shown in Figure 2-2. Physically, the source is a californiumpalladium cermet alloy, welded inside double stainlesssteel capsules. The source is certified Special Form by the U.S. Department of Transportation, and this certification simplifies its shipment by common carriers like FedEx.

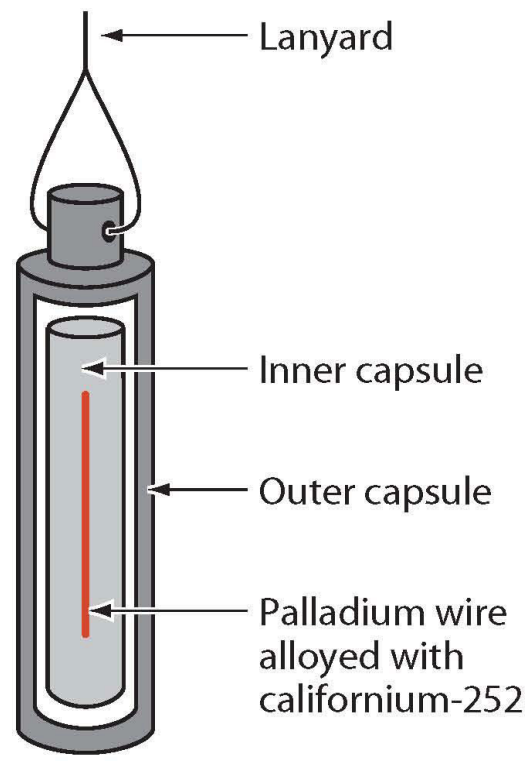

Figure 2-2: Cutaway view of a Cf-252 neutron source.

Figure 2-1: PINS equipment set. 


\section{Source/Moderator/Shadow-Shield Assembly}

The PINS Cf-252 source is placed in a slot in the polyethylene moderator block. Fast neutrons from the source directly irradiate the object under test. Fast neutrons that are emitted by the source in the direction away from the object under test will scatter and slow in the moderator, and some of the moderated neutrons will then diffuse to the object under test, as shown in Figure 2-3.

The tungsten shadow shield prevents gamma radiation from reaching the detector from the Cf- 252 source and the moderator block. The bismuth collimator reduces background gamma rays from reaching the detector; many of these gamma-rays are produced by neutron interactions in the ground at the measurement site. The combined effect of these shielding materials is improvement of the PINS spectrum signal-to-noise ratio.

The PINS equipment stand aligns the detector with the source, moderator, and shadow shield. The stand is equipped with both fixed and adjustable legs, to help

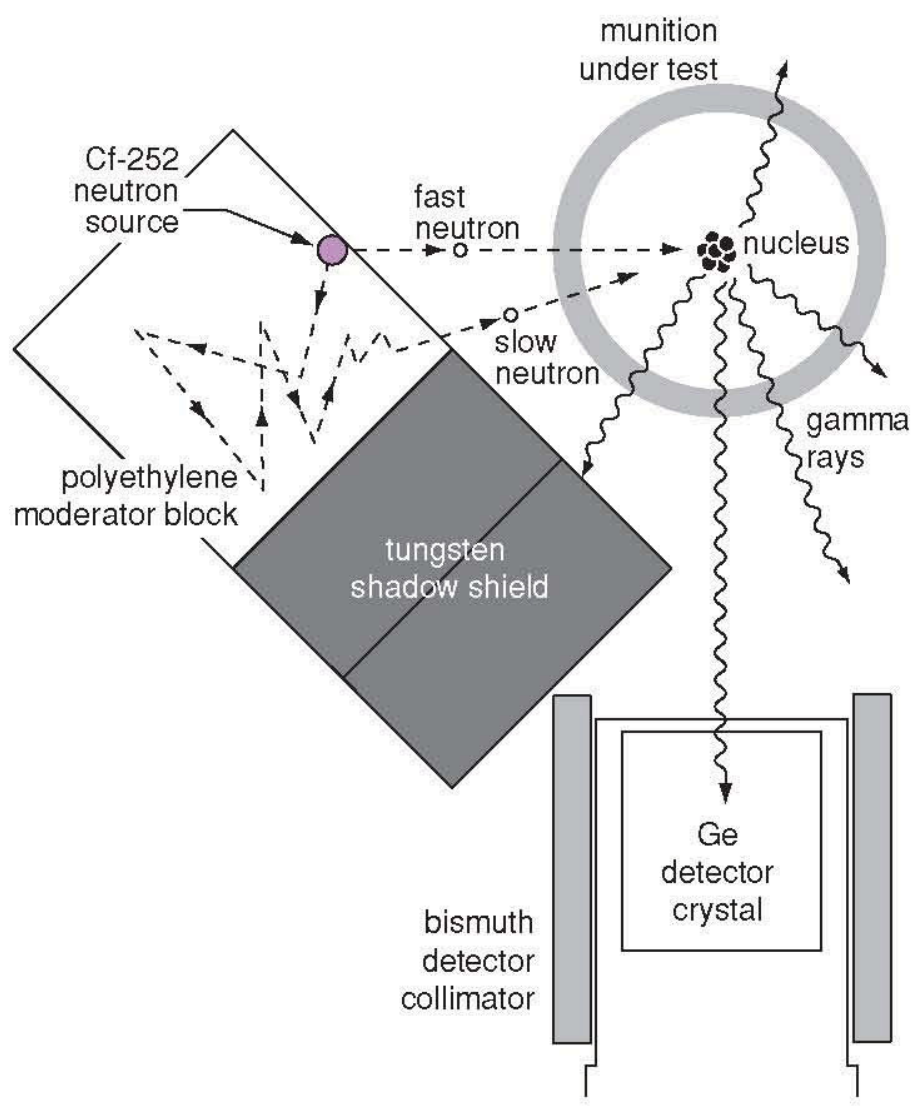

Figure 2-3: The PINS moderator block and shadow shield aligned with the gamma-ray detector. match the HPGe detector centerline to the height of the fill chemical center-of-mass within the object under test.

\section{HPGe Gamma-Ray Spectrometer}

The standard PINS gamma-ray spectrometer is a liquid nitrogen cooled $n$-type high-purity germanium (HPGe) detector of $40 \%$ relative efficiency. The detector cryostat is vacuum-insulated to minimize liquid nitrogen consumption, and its 1.2-liter liquid nitrogen dewar is sufficient to cool the detector for 18-24 hours. The detector also includes an integral high voltage power supply. An interlock disables the high voltage if the detector temperature exceeds its 120 Kelvin $\left(-243^{\circ} \mathrm{F}\right)$ setpoint, to protect the detector and its electronics if it runs out of liquid nitrogen.

A gamma-ray interaction in the detector crystal creates mobile electron-hole pairs, and these charges are swept to the detector electrodes by a strong electric field across the crystal. The amount of charge in a 50-nanosecond-wide pulse is directly proportional to gamma-ray energy. The detector preamp converts the charge pulse to a voltage pulse, and amplifies it for transmission through a cable to the multichannel analyzer.

\section{Multichannel Analyzer and Notebook Computer}

The multichannel analyzer, or MCA for short, is in effect a very high-speed voltmeter. The voltage of a pulse from the detector is directly proportional to gamma-ray energy. For example if a $1.0-\mathrm{MeV}$ gamma ray produces a 1.0 -volt pulse, a $2.0-\mathrm{MeV}$ gamma ray will produce a 2.0 volt pulse, and a $10.0-\mathrm{MeV}$ gamma-ray will produce a 10.0 -volt pulse. Hence the problem of measuring gamma-ray energies is reduced to the problem of measuring pulse voltages. The MCA analogto-digital converter (ADC) circuit measures the pulse voltage to a precision of one part in 8,192 , in just 5 microseconds.

The ADC passes its pulse voltage measurement to a histogram memory, in the form of a 13-bit binary number, that is, a number between decimal 0 and decimal 8,191. If the full-scale voltage is 10.0 volts, a 10 volt pulse would be assigned the value 8191 . A 5.0-volt half-scale pulse would be measured by the value 4,095-half of 8191 after rounding. The histogram memory would then increment the number of counts in memory address 4,095 by one. Each histogram memory address is termed a "channel", hence the name "multichannel analyzer".

The spectrum is written in the MCA histogram memory by the ADC, and the spectrum can be readout by an attached computer for display, analysis, and storage. 


\section{PINS Software}

A notebook computer functions as the PINS control panel, and INL's PINS + software controls both data acquisition and data analysis. During data acquisition, data analysis runs concurrently.

\section{Control and Data Acquisition Software}

INL's PINS + computer program controls the instrument startup, data collection, and shutdown. Instrument startup and shutdown are carried out through a simple checklist. The PINS+ control screen is shown in Figure 2-4. The principal controls are the Start, Stop and Clear buttons, plus the Counting Time Preset buttons. Note the status information on the left side of the control panel, including the assay real time, live time, fractional dead time, detector high voltage, and detector temperature.

\section{Analysis Software}

PINS+ also contains software to analyze spectra and determine the chemical fill of an item. This software operates in two modes. During data acquisition, the data analysis programs executes about once every ten seconds, updating the fill-identification box on the control panel shown in Figure 2-4. During data replay, it analyzes spectra in the file browser as clicked by the user, or automatically, if the user selects the Analyze All menu item of the Analyze menu.

PINS spectrum analysis will be discussed in detail in Chapters 3 and 4 .

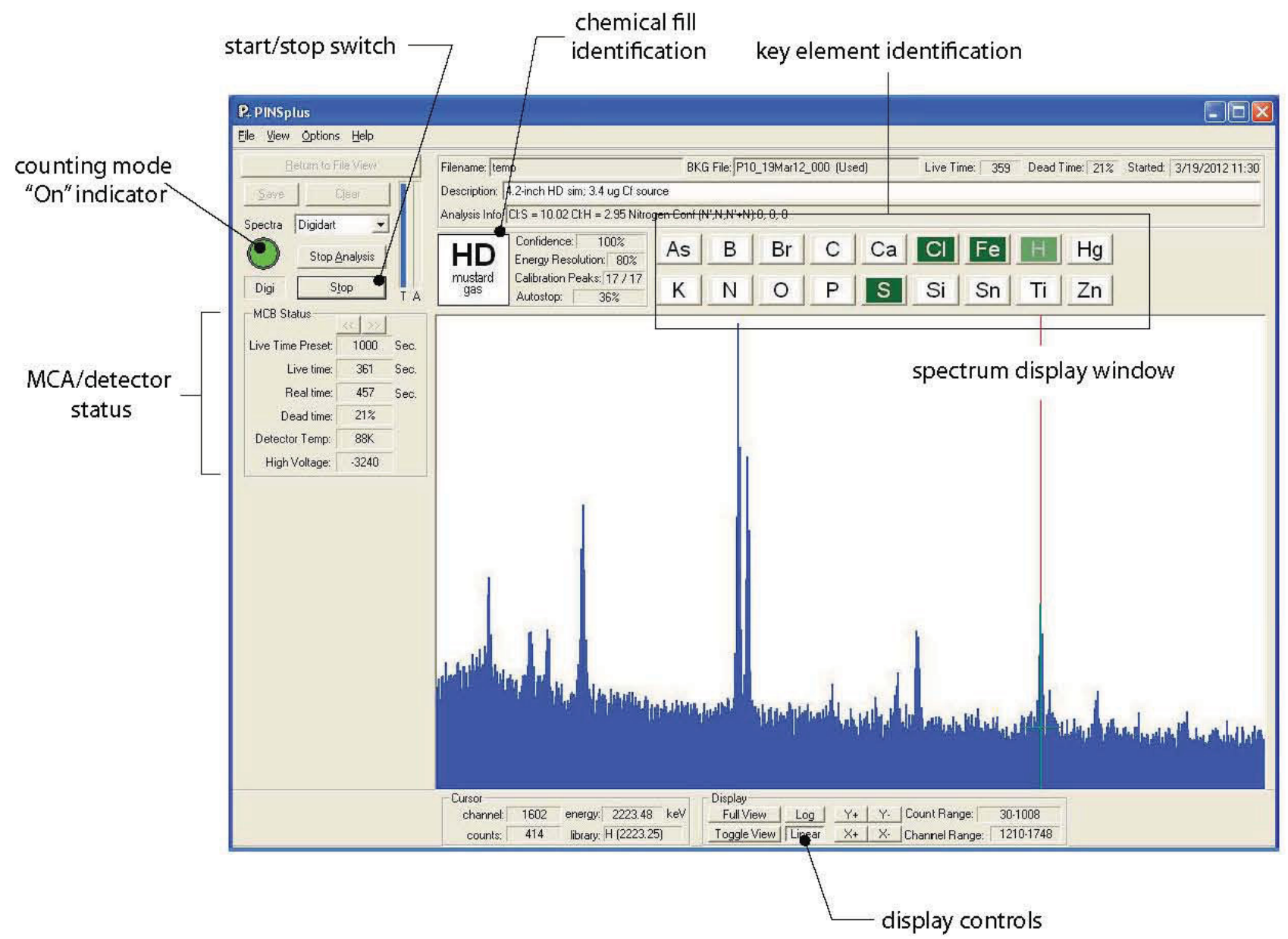

Figure 2-4: PINS+ control screen. 


\section{Operational Issues}

To collect a good-quality PINS spectrum that is easy to interpret, the measurement must be conducted with care. Some of the PINS operational issues are discussed in the next few sections.

\section{Backgroundfrom the Environment}

The PINS Cf-252 source emits neutrons isotropically, and the neutrons that miss the object-under-test can interact with chemical elements in the ground or a nearby building wall. As examples, PINS spectra recorded on a Dugway Proving Ground salt flat will display moderate chlorine peaks, no matter the actual fill chemical, from neutron interactions in the salt; PINS spectra of projectiles resting on sand bags will feature very strong silicon peaks; and munitions assayed atop of shipping pallets will have atypically strong hydrogen peaks from the moisture in wood.

It is not always possible to set up PINS in an area free of chemicals, and most PINS assays measure the presence of hydrogen and silicon in soil or concrete. Hence PINS operators must record a background spectrum with nothing in front of the detector for 1,000 seconds prior to assaying a container or munition. The PINS + software automatically subtracts the background, element by element, from the spectrum measured from a suspect object.

\section{Background from the Object-Under-Test}

An M121 155-mm artillery projectile has a fill weight of approximately 10 pounds, and the forged steel projectile weighs 80 pounds empty. The most prominent features in most PINS spectra from steel projectiles are the iron capture and inelastic gamma-ray peaks, and these peaks constitute a background of their own. The energy resolution of the PINS HPGe detector allows one to distinguish gamma rays from the chemical fill from the projectile's iron gamma rays. Figure 2-5 shows a nerve agent sulfur gamma-ray peak amid two iron gamma-ray peaks; the cleanly resolved 5421-keV sulfur peak and the $5409-\mathrm{keV}$ iron single-escape peak are separated in energy by just $0.22 \%$. The PINS + software uses the numerous iron gamma-ray peaks in PINS spectra to automatically calibrate the instrument's energy scale.

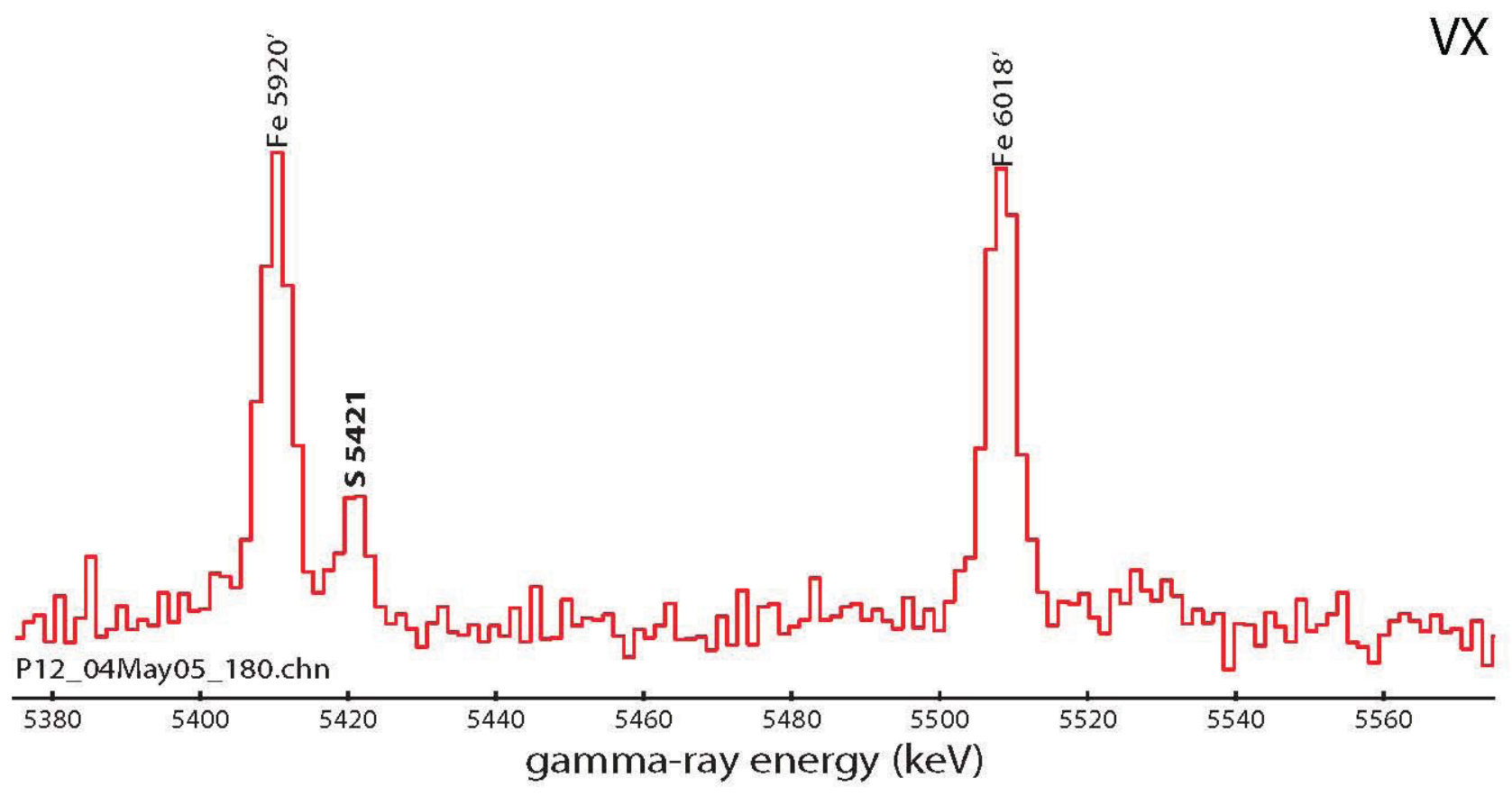

Figure 2-5: The VX 5421-keV sulfur peak and neighboring iron peaks. 


\section{Energy Resolution}

As mentioned above, detector energy resolution is important to distinguish gamma-ray peaks closely spaced in energy. It's also important for the identification of weak peaks sitting on a large background, as seen in Figure 2-6.

Energy resolution can be compromised by several factors, including electrical noise, excessive counting rates, detector moisture, and thermal effects. Whenever possible, avoid connecting a PINS system to a circuit powering electric motors or other devices that create electrical noise. The detector is not waterproof, and if a detector must be operated in inclement weather, protect it inside a plastic bag.
The detector takes 4-6 hours to cool down from room temperature to operating temperature. However, when a detector first reaches operating temperature, it continues to cool, and its output is not really stable until its temperature becomes constant. It's best to allow a detector to cool overnight prior to use, to avoid resolution loss due to temperature instability.

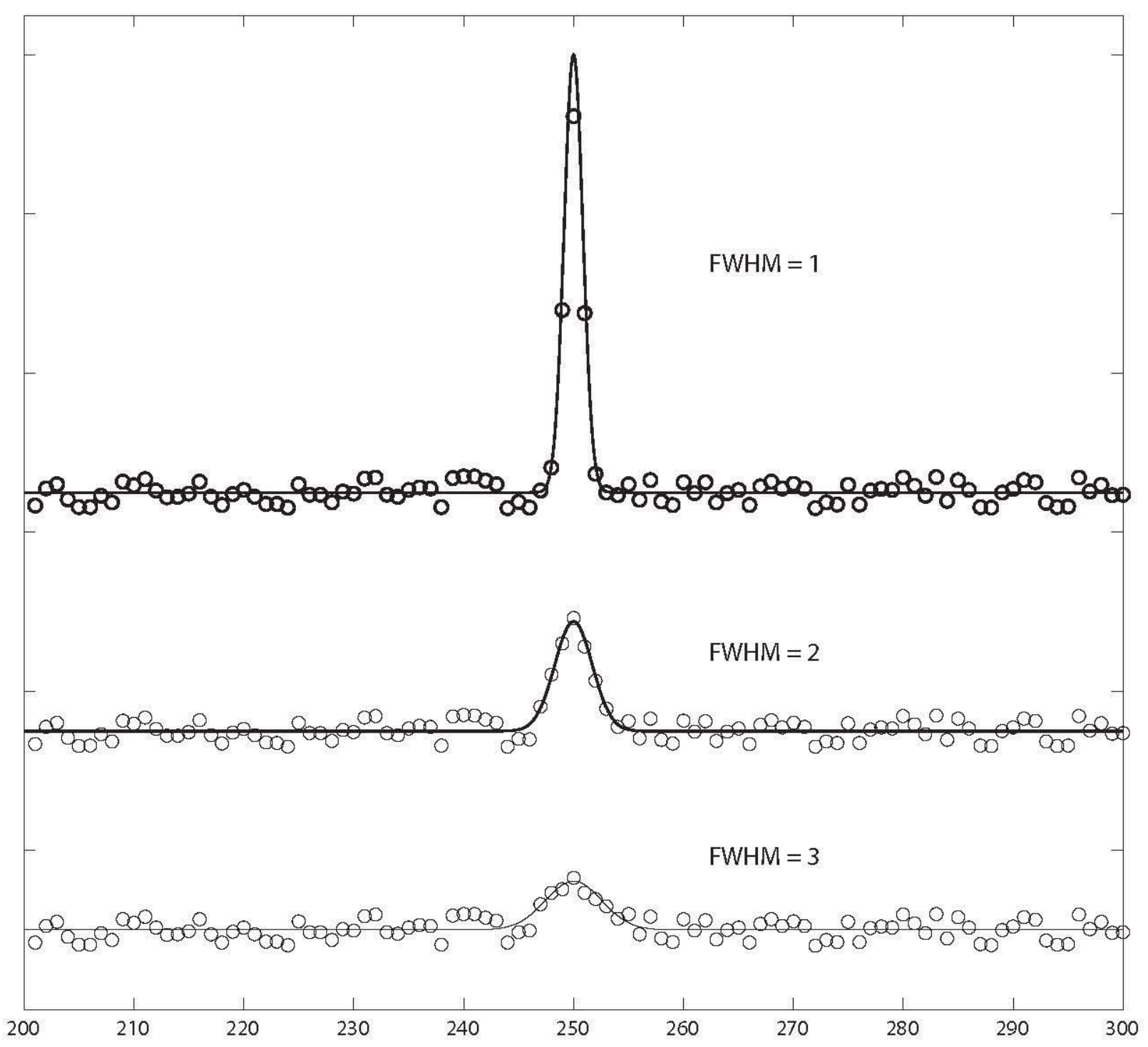

Figure 2-6: Resolution effects on peak amplitudes. The net peak area is the same for all three peaks, but the peak full widths at half-maximum (FWHM) are either 1, 2 or 3 channels. 
Operating a detector at very high counting rates can also hurt energy resolution. As the count rate increases, so does pulse pile-up, and the energy resolution is reduced. Pulse pile-up occurs when one pulse rides up on the tail of an earlier pulse, as shown in Figure 2-7.[1] The instrument's fractional dead time is proportional to its counting rate, and by keeping the dead time less than $35 \%$, pile-up effects can be minimized.

\section{Assay Duration}

The counting interval needed to identify a given chemical varies from 100 seconds for some types of smoke chemicals to over 1,000 for explosives like TNT. Since the fill chemical is initially unknown, the operator usually sets up PINS to count for at least 1,000 seconds, and commonly for 3,000 seconds.

\section{Futher Information for Operating the PINS Instrument}

This brief chapter is simply an overview, and it is no substitute for the detailed operating instructions found in the PINS User's Manual. [2]

\section{References}

1. After Figure 1 in K. Taguchi et al., "An analytical model of the effects of pulse pileup on the energy spectrum recorded by energy resolved photon counting x-ray detectors," Medical Physics 37 (2010) page 3958.

2. A.L. Siedenstrang et al., PINS Chemical Assay System User's Manual version 4.7, Idaho National Laboratory Report INL/EXT-08-15141 (May 2009).

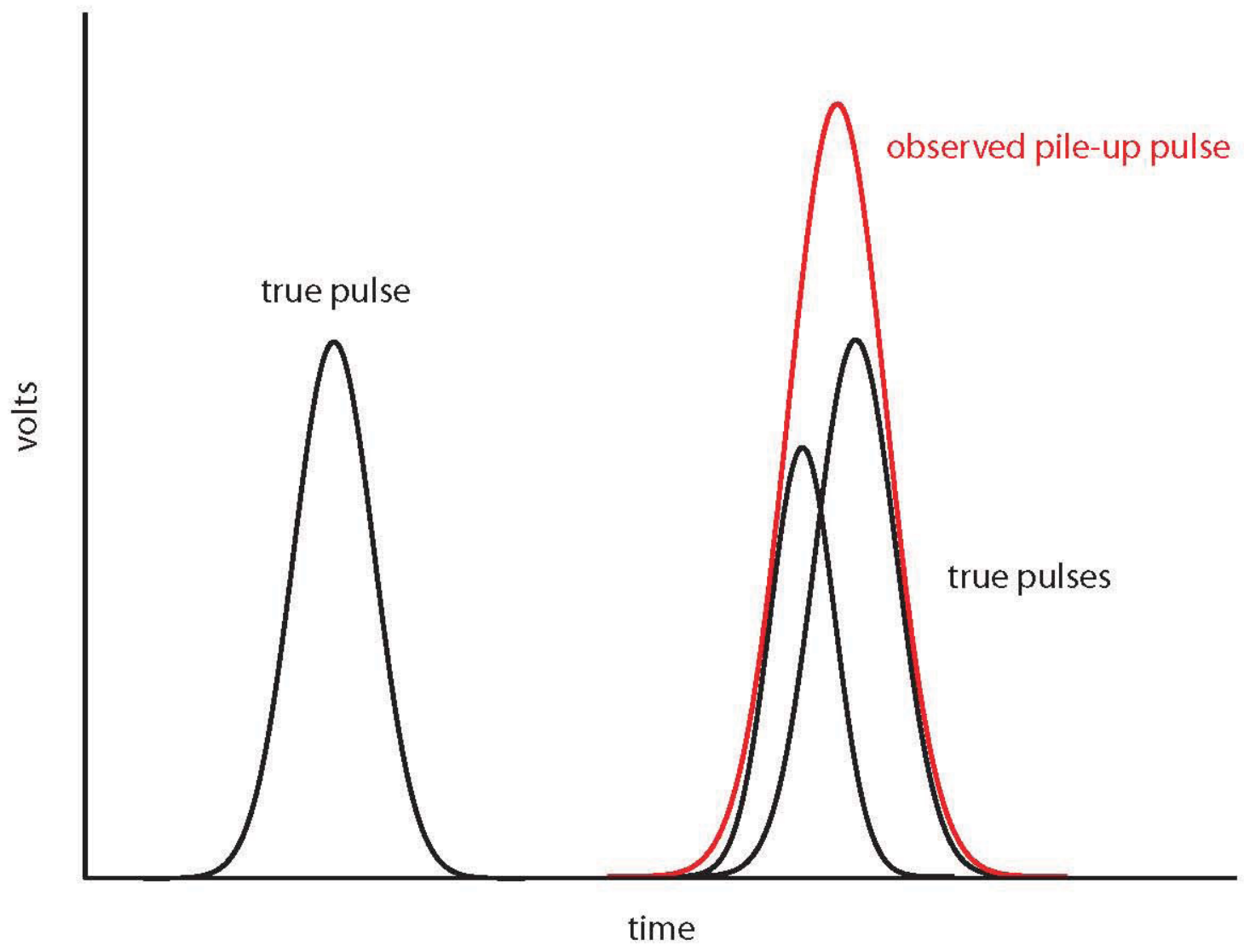

Figure 2-7: Pulse pile-up effect. This effect is most pronounced at high counting rates. 


\section{Chapter 3}

\section{Spectrum Analysis Basics}

A $t$ the beginning of the twentieth century, atomic spectra were an unexplained problem. Why did mercury placed in an electric arc produce green light, when sodium produced yellow light? Why don't excited atoms produce the entire rainbow of colors? Each chemical element seemed to have its own set of characteristic optical spectral lines or peaks, at reproducible wavelengths. Classical theory could not explain any of these phenomena.

In 1913, Neils Bohr provided the answer. The energy levels of electrons bound to an atomic nucleus are restricted or "quantized", that is, only certain energy levels are permitted by what's now known as quantum mechanics.[1] The energies of the emitted light are limited to differences between the quantized energy levels. Bohr's Nobel Prizewinning paper only addressed the atomic levels of hydrogen atoms, but his insights turned out to be applicable to atoms, molecules, and atomic nuclei. Today, the characteristic energy levels of atomic nuclei are well known.

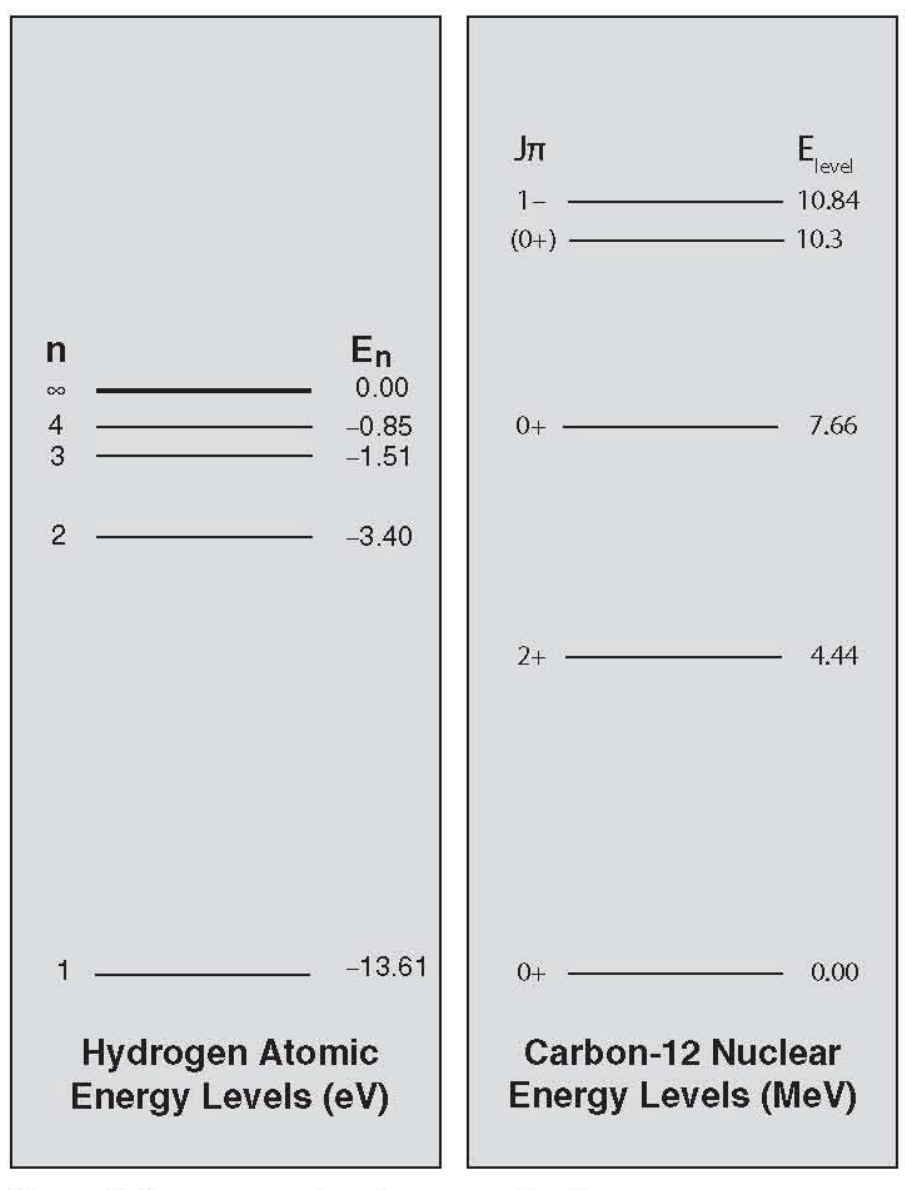

Figure 3-1: Atomic and nuclear energy levels.
Figure 3-1 compares the hydrogen atomic energy levels with those of carbon-12 nuclei; both are clearly quantized. Note the nuclear energy levels are spaced about one million times farther apart than atomic levels. Transitions between nuclear levels fall not in the optical, but in the gamma-ray portion of the electromagnetic spectrum.

When a neutron is captured by a nitrogen-14 nucleus, a highly excited nitrogen-15 $\left({ }^{15} \mathrm{~N}\right)$ nucleus is produced, and this nucleus de-excites rapidly[2] by gamma-ray emission. The energy levels and gamma-ray transitions of ${ }^{15} \mathrm{~N}$ are shown in Figure 3-2. The 10829-keV gamma ray indicated by the blue arrow in the figure is used in PINS spectral analysis to identify the presence of nitrogen in the object under test.

This chapter will examine the fundamentals of gamma-ray spectroscopy. These principles will be applied to the chemicals commonly identified by PINS in the following chapters.

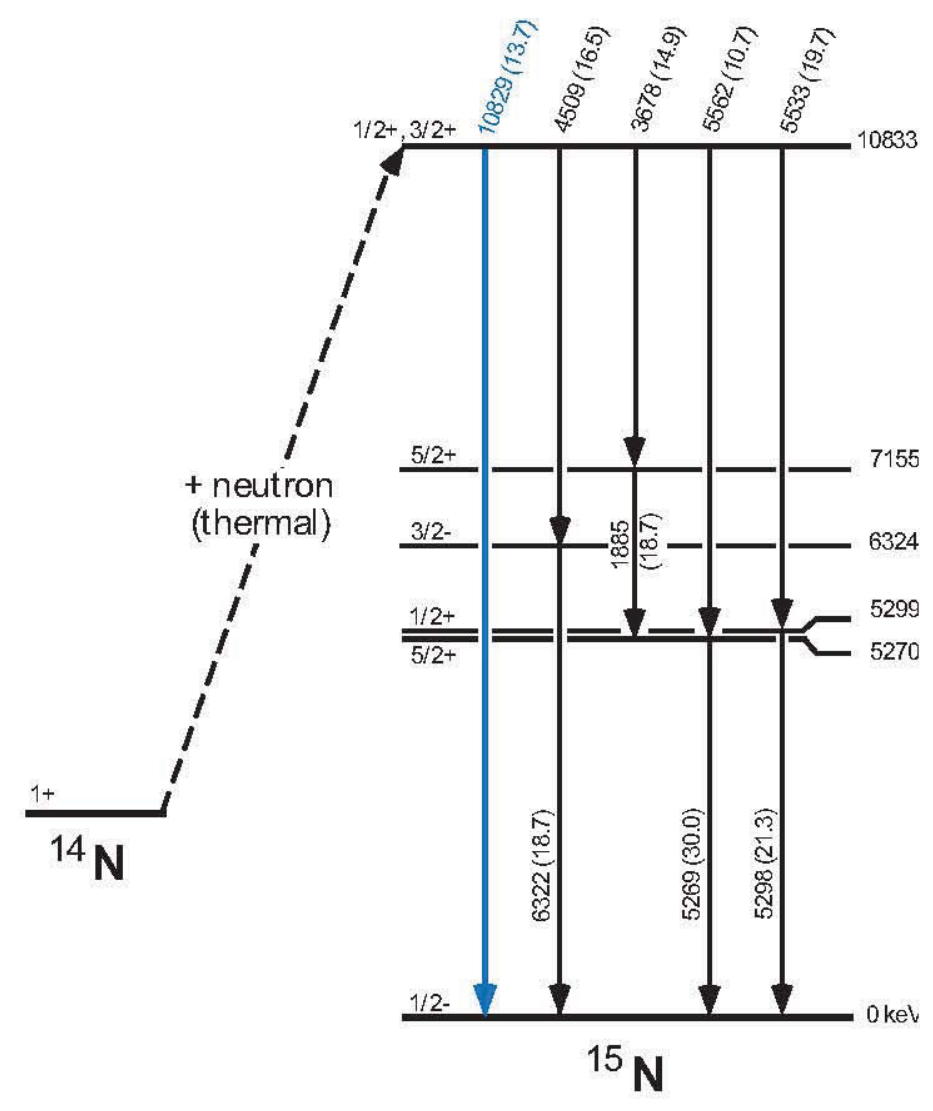

Figure 3-2: Nitrogen-15 nuclear energy levels. 


\section{Gamma-Ray Peak Evaluation}

The analysis of PINS gamma-ray spectra begins with the analysis of the gamma-ray peaks.

\section{Peak Energy and Chemical Identification}

Figure 3-3 shows the 661.7-keV cesium-137 gamma-ray peak atop a background continuum. For an isolated peak, as in the figure, the centroid $x_{c}$ can be computed by summing:

$$
x_{c}=\Sigma x \cdot y(x) / \Sigma y(x),
$$

where $x$ is a channel number, $y(x)$ is the number of counts in channel $x$, and the summations are carried out over the channels in the peak. The centroid will not always coincide with the peak channel with the most counts, but it will not be far away.

Once a spectrum has been energy calibrated, the centroid energy $\mathrm{E}_{\mathrm{c}}$ may be computed from the energy equation,

$$
E_{c}=a+b x_{c}+c x_{c}^{2} \text {. }
$$

The peak centroid and full width at half-maximum (FWHM) were determined in Figure 3-3a by summing methods.

The peak centroid energy identifies the isotope, and hence the chemical element, that emitted the gamma ray. In the literature, there are tables of gamma-ray energies for radionuclides, [3] capture gamma rays,[4] and inelastic scattering gamma rays. [5] A table of gamma-ray energies for PINS spectra can be found in Appendix B starting on page 107.

PINS+ identifies the energies of capture and inelastic gamma-ray peaks directly. The user simply places the cursor on a peak and reads out the peak energy at the bottom left of the control window. If the peak energy is in the PINS gamma-ray library, the emitting chemical element is identified as well.
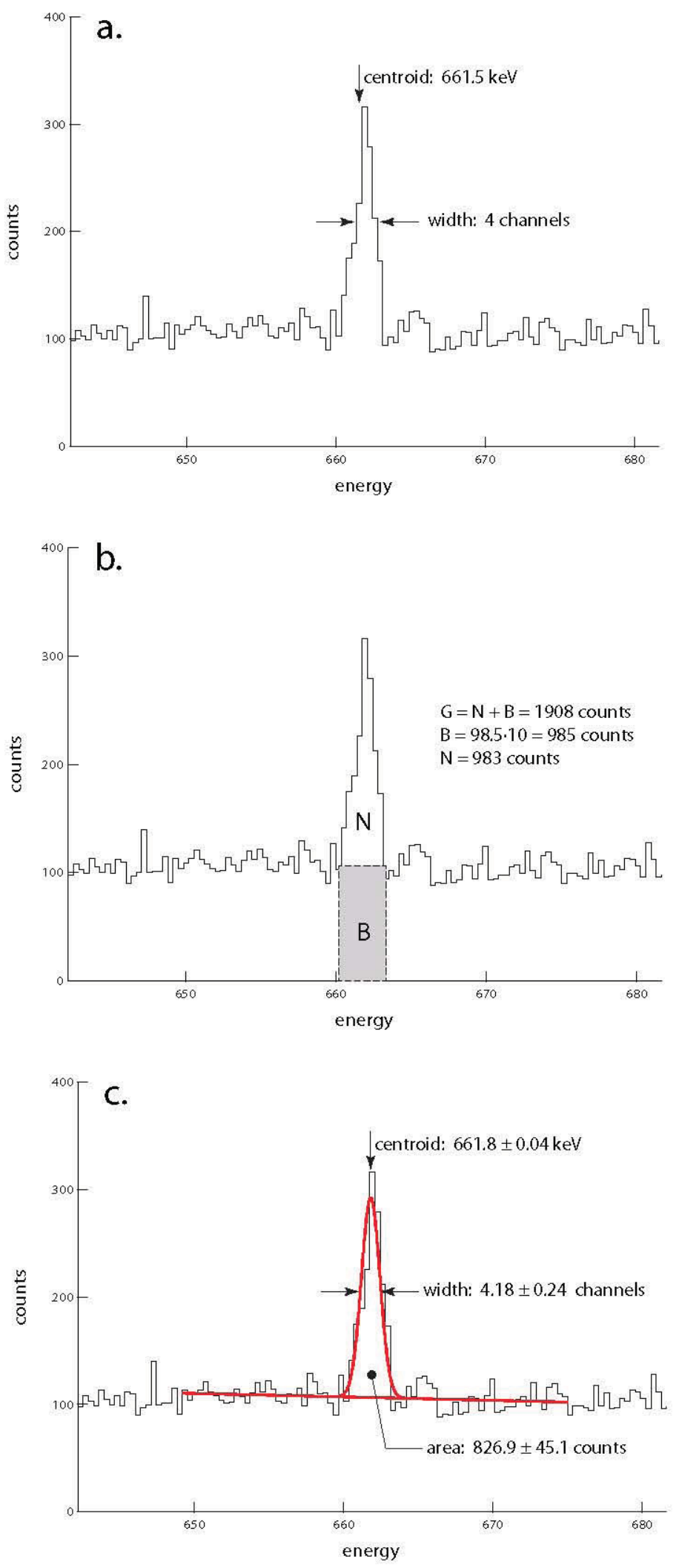

Figure 3-3: Cs-137 gamma-ray peak. a. peak centroid and width determined by summing methods. $b$. peak area determined by summing methods. c. peak centroid, width, and area determined by non-linear least-squares fit. 


\section{Energy Calibration}

The energy equation converts spectrum channel numbers to energies. In the example above, the energy equation is quadratic, to correct for a slight nonlinearity in the spectrometer electronics. A linear relation often suffices, although PINS+ uses a quadratic equation. The energy equation is determined by fitting a curve to a plot of gamma-ray energies vs. gamma-ray centroid channels, as shown in Figure 3-4.

PINS+ automatically calibrates the spectrum energy scale. When collecting a new spectrum, calibration frequently requires $60-100$ seconds, until a sufficient number of calibration peaks has been found by the peak search algorithm. When the number of calibration peaks is ten or greater, the PINS+ energy calibration is usually excellent.

\section{Peak Area and Background}

The gamma-ray peak of Figure 3-3a is displayed again in Figure 3-3b. Next to its energy, a peak's most important attribute is its area in counts. Unlike the peaks in mass spectrometers, gamma-ray peaks ride on a continuum background, and this background must be subtracted from the gross peak area to determine the true, or net peak area.

The simplest method of determining peak areas is summing. [6] The gross peak area $G$ is the sum of counts per channel from one side of the peak to the other, and its uncertainty, since gamma-ray counting obeys Poisson statistics, is

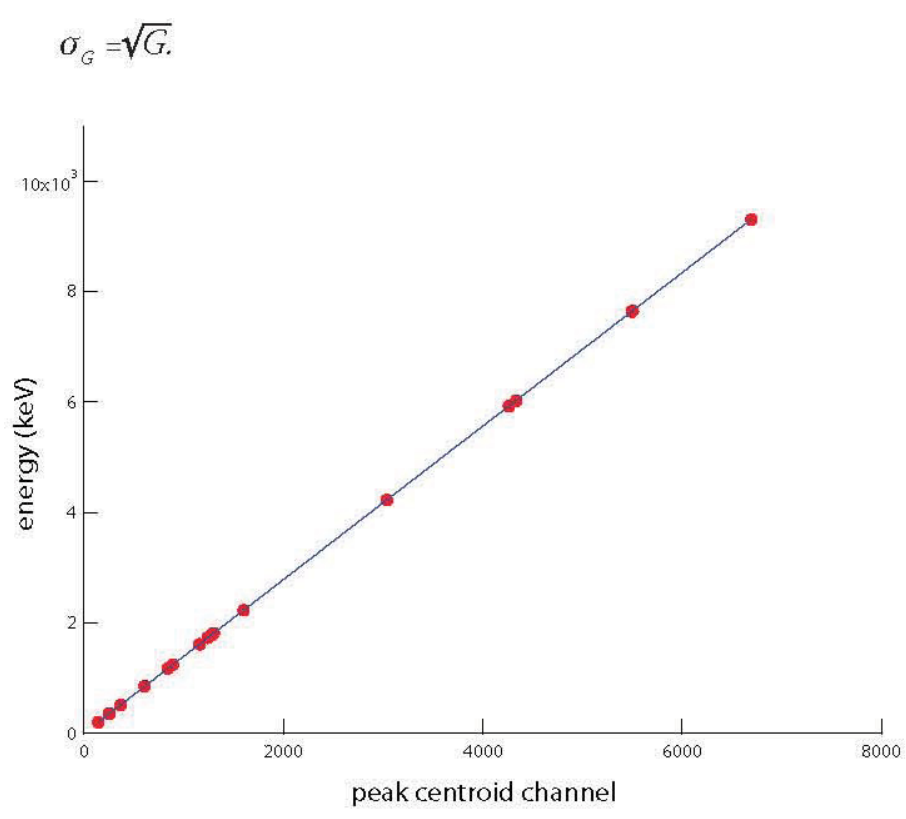

Figure 3-4: Least-squares fit of gamma-ray energies to gamma-ray peak centroids.
The continuum background $B$ is estimated from the channels to the left and right of the peak, and its uncertainty is

$$
\sigma_{B}=\sqrt{B}
$$

The net peak area is $\mathrm{N}=\mathrm{G}-\mathrm{B}$, and its uncertainty is

$$
\sigma_{N}=\sqrt{\sigma_{G}^{2}+\sigma_{B}^{2}}=\sqrt{G+B}=\sqrt{N+B+B}=\sqrt{N+2 B} .
$$

Note that the background uncertainty is especially import for small peaks where $N<B$.

\section{Peak Fitting vs. Peak Summing}

As noted above, peak centroids and peak areas can be found by summing methods. Another approach to gamma-ray peak parameter determination is peak fitting. Figure 3-3c displays a nonlinear least-squares fit of a Gaussian function to the gamma-ray peak of Figure 3-3a. A line segment was also fit to the continuum background. Peak fitting determines the centroid, net peak area, and the related uncertainties in one operation. [7]

While summing methods are quite accurate for isolated peaks, the analysis of closely-spaced overlapping peaks requires nonlinear least-squares peak fitting. A fit of multiple gamma-ray peaks from the hydrogen region of a PINS spectrum is shown in Figure 3-5.

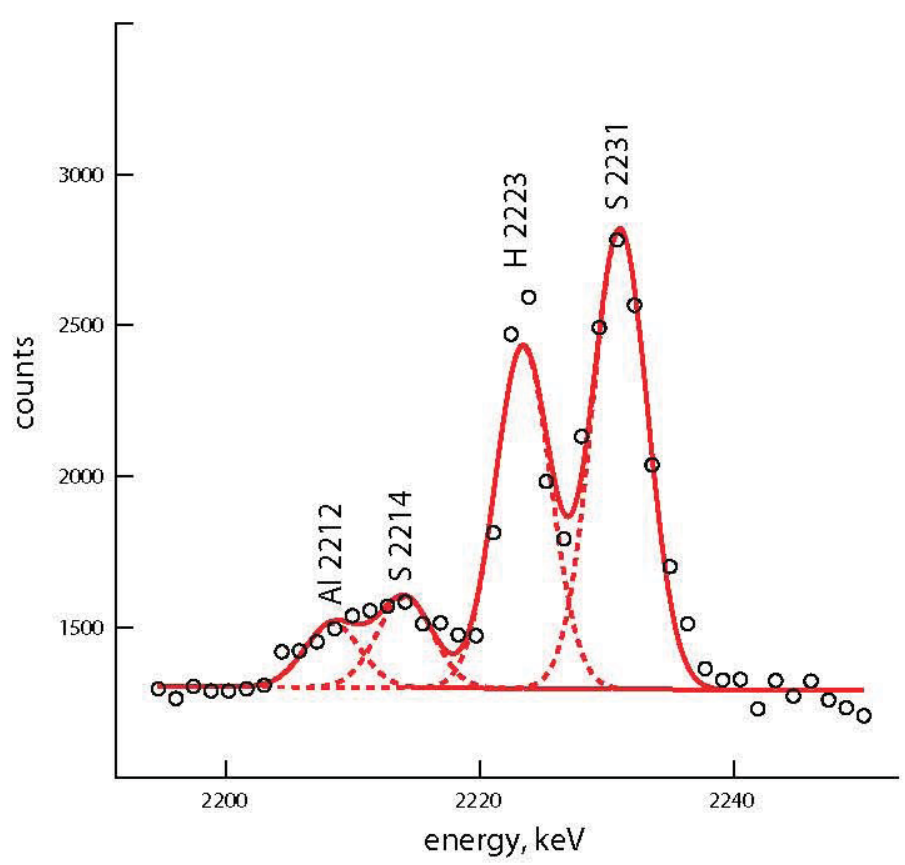

Figure 3-5: Four-Gaussian peak fit to the hydrogen region of a PINS FS smoke spectrum. 
PINS+ uses nonlinear least-squares peak fitting methods whenever possible. The usual exceptions are peaks of irregular shape like the boron- 10 peak at $477 \mathrm{keV}$, and the relatively weak nitrogen peaks at 10.3 and $10.8 \mathrm{MeV}$. In PINS+, the latter three peaks are measured by summing.

\section{Detection Confidence}

A visible spectral bump at the energy of a known gamma ray suggests the presence of the related chemical element; however the bump can be either a fluctuation in the continuum background or a real effect. A more quantitative approach is to compute the ratio $t^{*}$ of the net peak area to the net peak area uncertainty. From the statistical properties of the cumulative normal probability distribution, [8] one can compute the peak detection confidence level vs. $t^{*}$, and this relation is displayed in Figure 3-6. PINS+ uses this concept to determine the detection confidence level for each gamma-ray peak of interest.

\section{Identification of Chemicals}

A few of the munitions and containers assayed with PINS contain a single chemical element, but usually, multiple chemical elements must be evaluated to determine the fill of the object-under-test.

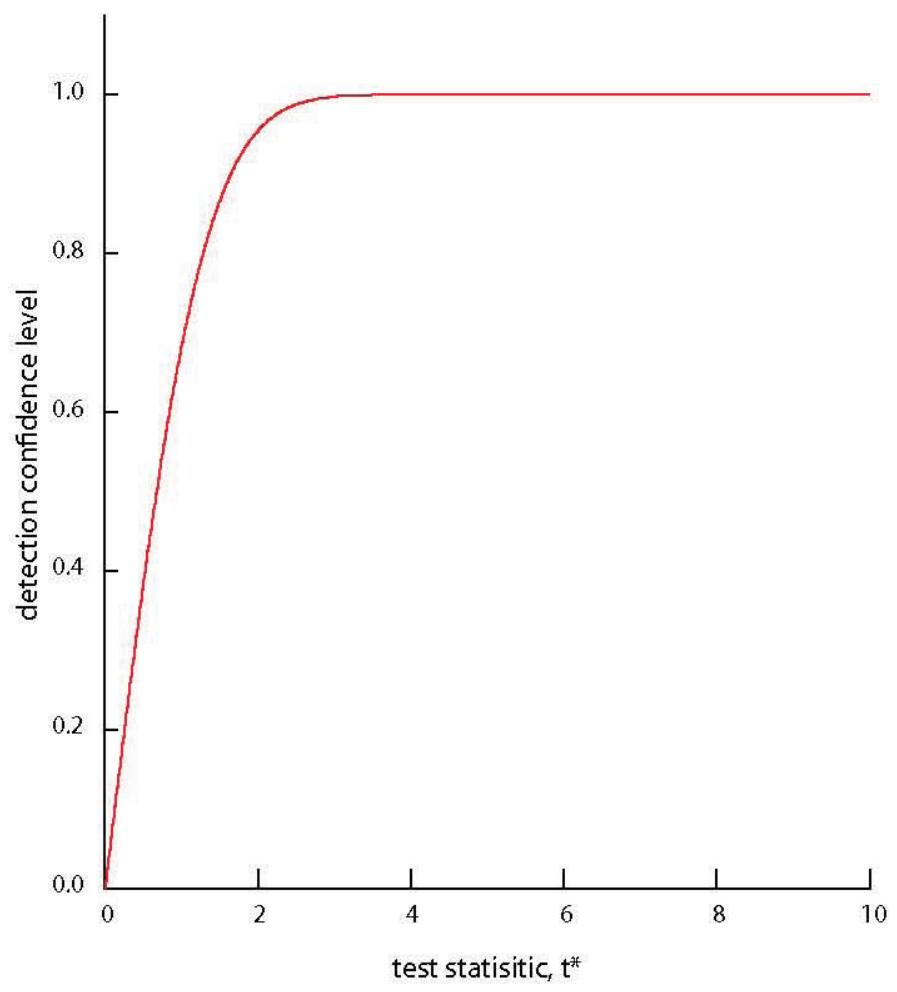

\section{Key vs. Common Chemical Elements}

Selected chemical elements, like phosphorus, are considered key elements in PINS analysis. These elements, singly or in combination, are used in the PINS identification logic to determine munition fill chemicals. The PINS key elements and the fill chemicals they identify are listed in Table 3-1.

Table 3-1: PINS key elements

\begin{tabular}{ll}
\hline Element & Found in \\
\hline arsenic & blood agent arsine, blister agent lewisite \\
boron & water/antifreeze practice fills \\
bromine & riot agent CA \\
calcium & decon bleach, plaster of Paris (POP) \\
& practice fill \\
chlorine & blister agents, smoke fills, riot agents \\
hydrogen & nerve agents, blister agents, riot agents, \\
& explosives, water/AF and POP practice \\
nitrogen & explosives, VX nerve agent \\
phosphorus & nerve agents, WP smoke \\
silicon & sand practice fills \\
sodium & decon bleach, table salt \\
sulfur & VX nerve agent, mustard agent, \\
tin & FS smoke, POP \\
titanium & KJ smoke \\
zinc & FM smoke \\
\hline
\end{tabular}

Table 3-2: PINS common chemical elements

\begin{tabular}{ll}
\hline Element & Gamma-ray energies $(\mathrm{keV})$ \\
\hline aluminum & 1014.4 \\
bismuth & 1609.0 \\
carbon & 4438.9 \\
germanium & 198.8 \\
indium & $416.9,818.6,1097.2,1293.5,2112.2$ \\
iron & $846.8,1238.3,7631.13,7645.45$ \\
tungsten & 228.8 \\
\hline
\end{tabular}

Figure 3-6: peak detection confidence vs. the $t^{*}$ statistic. 
Many chemical warfare agents are organic chemicals, that is, they contain the chemical element carbon. Carbon is also found in most military explosives, and it is present in inert substances like polyethylene as well. Hence carbon gamma rays are not used by PINS to identify munition fill chemicals, and carbon is considered a common element, as opposed to a key element. Table 3-2 lists the PINS common elements.

Other common elements include aluminum, bismuth, germanium, indium, and tungsten. These materials are found in the PINS HPGe detector and its stand and moderator/ shadow shield assembly. Stray neutrons from the Cf- 252 source produce a constant gamma-ray background from these elements, and their background level is minimized by the design of the detector collimator and shadow shield.

To view a PINS key element gamma-ray peak, simply click one of the display buttons labeled by the element's chemical symbol. If the key element has more than one prominent gamma-ray peak, clicking the button again moves the cursor to another peak. Clicking the button again will either move to a third peak or return to the original peak of the selected element.

\section{Decision-Tree Methods}

Originally PINS used a decision-tree method to identify fill chemicals, and a recent PINS decision tree is shown in Figure 3-7.

Basically, the decision tree requires a yes/no decision at each node as one traces a path to the fill. For example, if phosphorus peaks are not observed at 1266 and 2233 $\mathrm{keV}$, but the chlorine doublet peaks are seen at 1951 and $1959 \mathrm{keV}$, and a titanium peak is observed at $983 \mathrm{keV}$, the fill chemical is titanium tetrachloride, FM smoke, as the colored path in Figure 3-7 suggests.

Note also in Figure 3-7 that detection or non-detection of chlorine cuts the number of fill possibilities in half.

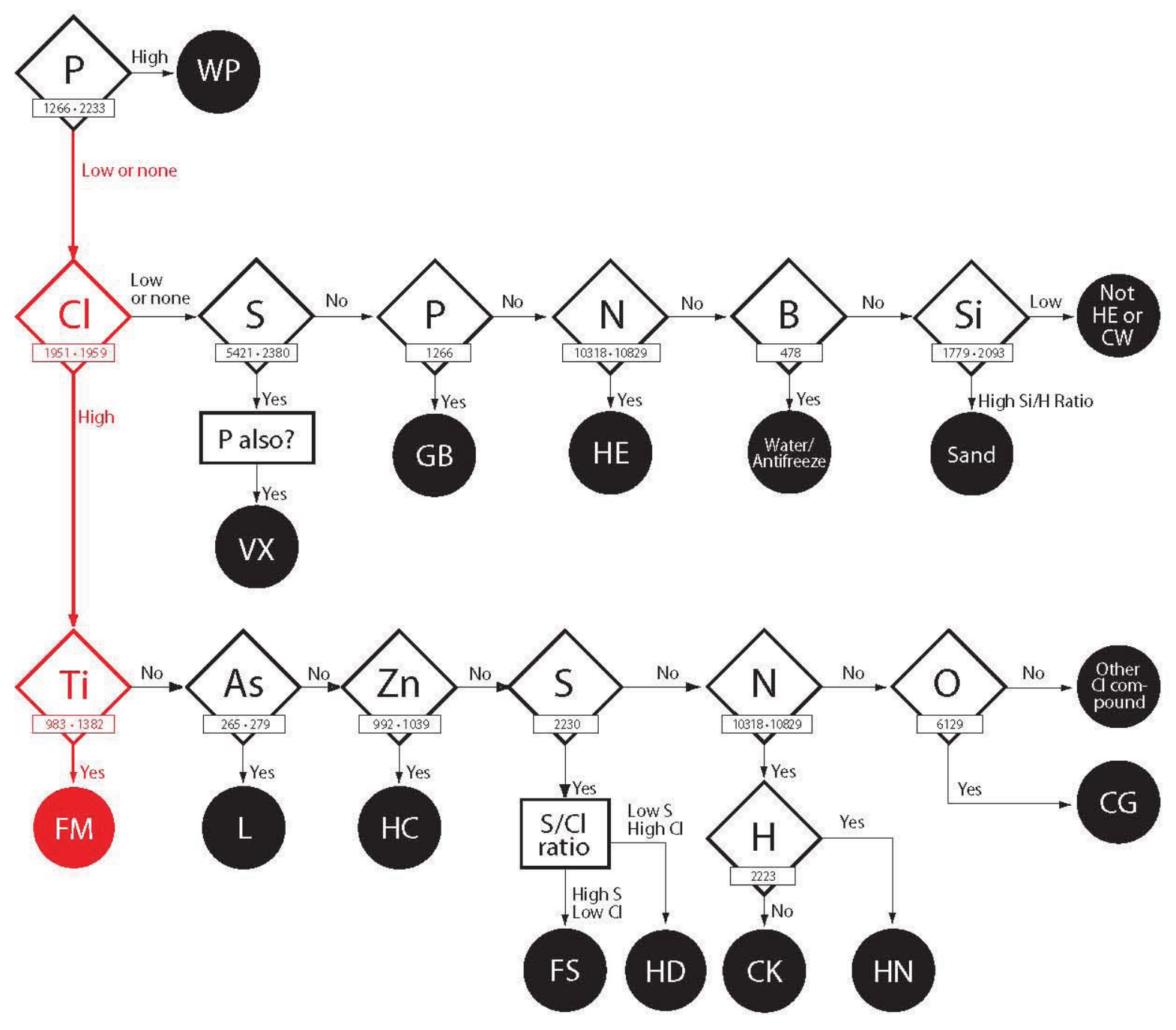

Figure 3-7: PINS decision tree. 


\section{Elemental ratio methods}

As the PINS decision tree was expanded to include additional chemicals, its yes/no nature reached its limits. Consider mustard agent (agent HD) and FS smoke. Both chemicals contain three PINS key elements: chlorine, hydrogen, and sulfur. It is not possible to differentiate between them based simply on the presence or absence of key elements.

Recently, ratio analysis methods have been added to the PINS chemical identification logic. Figure 3-8 compares the chlorine, hydrogen, and sulfur peaks between mustard agent and FS smoke. Note that for FS, the 1959-keV chlorine peak is approximately the same size as the 2230$\mathrm{keV}$ sulfur peak, while for agent HD the 1959-keV chlorine peak is approximate twelve times larger than the sulfur peak. The chlorine-to-sulfur ratio computed from the areas of the 1959- and $2230-\mathrm{keV}$ peaks is a reliable method for distinguishing mustard agent from FS smoke.

The PINS+ uses a mixture of decision-tree and elementalratio considerations to identify fill chemicals, as will be explained in the following chapters.

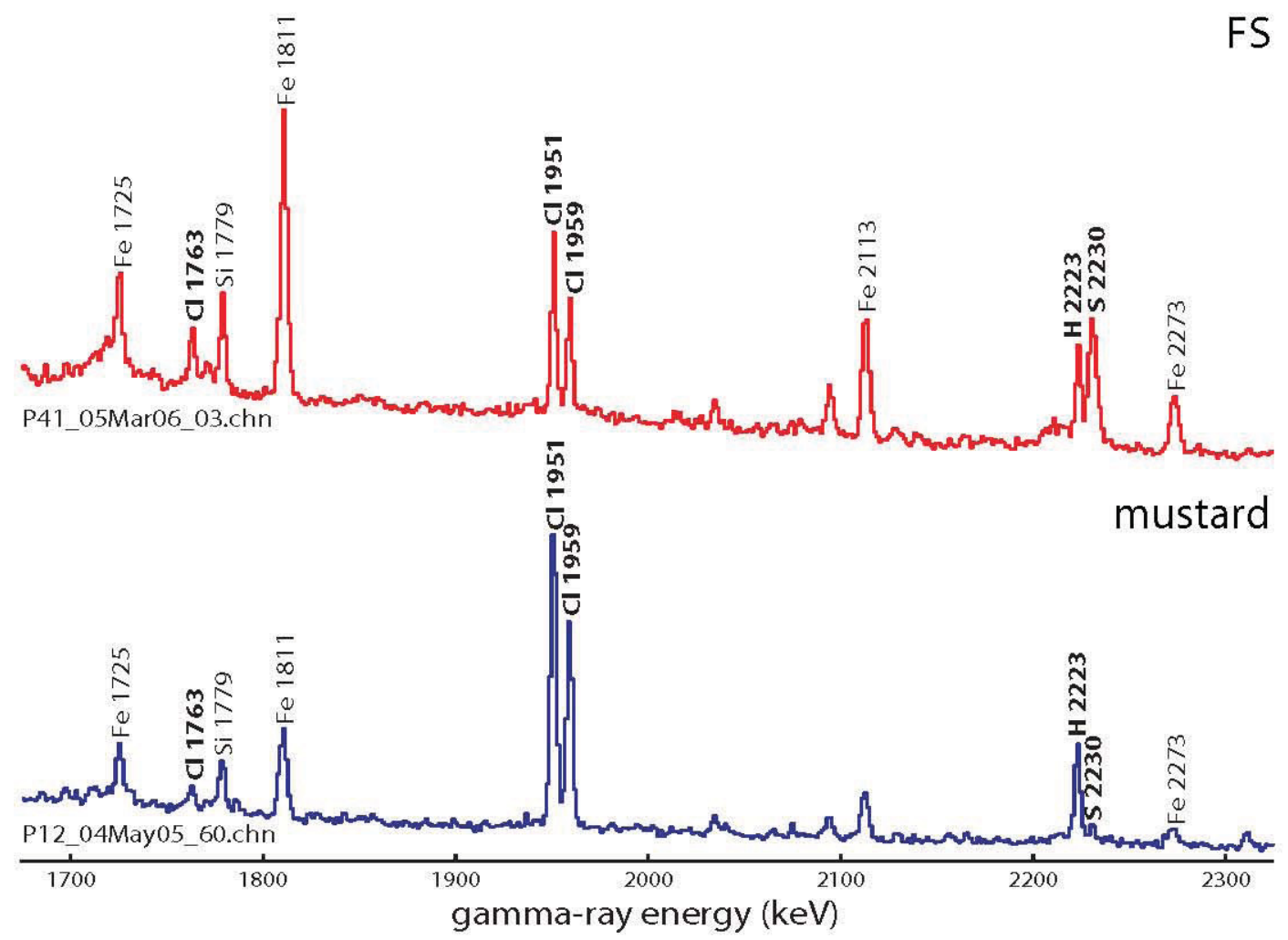

Figure 3-8: Comparison of the FS and mustard hydrogen spectral regions. 


\section{References}

1. The gentlest introductions to quantum mechanics are modern physics textbooks. See for example R. Harris, Nonclassical Physics (Menlo Park, CA: Addison-Wesley, 1998). The recent text by Christopher Hill and Nobel laureate Leon Lederman, Quantum Physics for Poets (Amherst, New York: Prometheus Books, 2011) offers a humorous, literate, and non-mathematical overview of the subject.

2. Nitrogen-15 de-excites after neutron capture in a few picoseconds. K. Debertin and R.G. Helmer, Gammaand X-Ray Spectrometry with Semiconductor Detectors (Amsterdam: Elsevier Science, 1988) pages 24-29.

3. G. Erdtmann and W. Soyka, The Gamma Rays of the Radionuclides (Weinheim, Germany: Verlag Chemie, 1979). The CEA-Saclay Nucleide-LARA website is a convenient on-line radionuclide gamma-ray energy table, and it is found at this link: http//laraweb.free.fr A shorter table of gamma rays commonly used for energy calibration can be found in Debertin and Helmer, pages 351-367.

4. M.A. Lone, R.A. Leavitt, and D.A. Harrison, "Prompt Gamma Rays from Thermal-Neutron Capture", Atomic Data and Nuclear Data Tables, 26, (1981) pages 511-559. G.L. Molnar, Ed., Handbook of Prompt Gamma Activation Analysis (Dordrecht, The Netherlands: Kluwer Academic Publishers, 2004). National Nuclear Data Center's "CapGam" is a convenient on-line capture gamma-ray website: www.nndc.bnl.gov/capgam/

5. A.M. Demidov et al., Atlas of Gamma-Ray Spectra from the Inelastic Scattering of Reactor Fast Neutrons (Moscow: Atomizdat, 1978).

6. G.G ilmore, Practical Gamma-Ray Spectrometry (Chichester, United Kingdom: John Wiley \& Sons, 2008) pages 109-111.

7. Debertin and Helmer, pages 54-69, 291-303.

8. J.R. Taylor, An Introduction to Error Analysis: The Study of Uncertainties in Physical Measurements, Second Edition (Sausalito, CA: University Science Books, 1997) pages 135-137. A. Jeffrey, Handbook of Mathematical Formulas and Integrals (San Diego, CA: Academic Press, 1995) pages 235-237. 
24 PINS Spectrum Identification Guide 


\section{Chapter 4}

\section{PINS Spectrum Analysis Overview}

$\mathrm{I}$ dentification of the chemical fill of an item assayed with PINS rests on correct interpretation of the gamma-ray spectrum produced during neutron bombardment. The first step in PINS spectrum analysis is calibration of the spectrum energy scale, and this is performed automatically by the PINS + software using gamma rays common to most PINS spectra, e.g. gamma rays from aluminum, bismuth, germanium, iron, hydrogen, and silicon. The next step is to identify and quantify the gamma rays of PINS key elements. Each element is identified by the energy of one or more gamma-ray peaks. The area of each key-element gammaray peak is directly proportional to the concentration of that element inside the item under test. The third step is to identify the fill chemical based on the presence and absence of the key elements, and their relative concentrations.

\section{Spectrum ID Chapter Organization}

The computer-based analysis of PINS spectra uses decisiontree logic, and it is convenient to arrange the spectrum identification chapters in a similar fashion. Accordingly, these chapters are organized by chemical element, like the trunk of the PINS decision tree shown in Figure 4-1.

Phosphorus-based chemicals, perhaps the most dangerous of all discussed in this Guide, come first; and they are followed by arsenic-based chemicals, chlorine-based chemicals, a bromine-based chemical, nitrogen-based explosives, and common practice-munition fills, like sand. In addition, the Guide includes an appendix on the identification of selected toxic industrial chemicals.

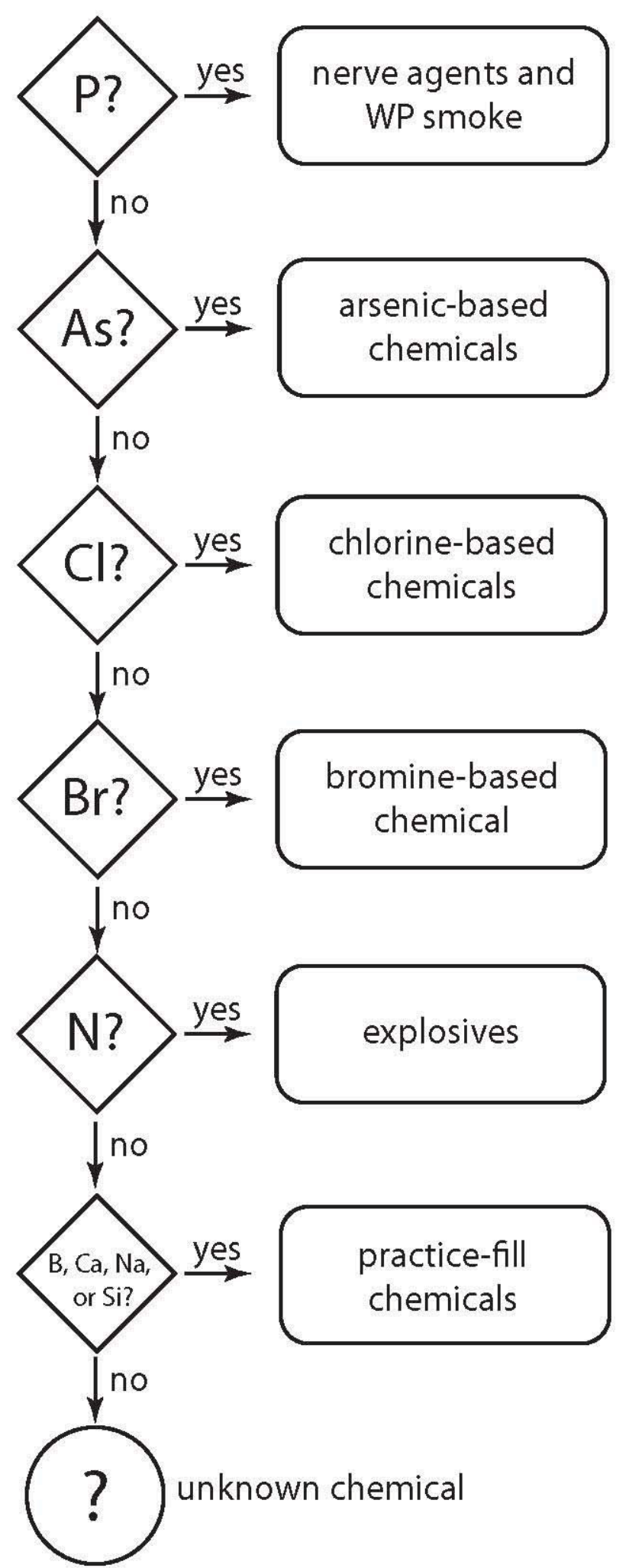

Figure 4-1: PINS decision tree trunk. 


\section{Decision Tree Branches}

Each chapter focuses on the chemicals identified by a specific decision tree branch. The opening pages of each chapter discuss the identification logic for all of the chemicals in the branch, and these pages are followed by spectrum identification sheets for each chemical.
The identification logic is similar to a computer flow chart, with the fill chemicals identified in circles, as shown in Figure 4-2. If PINS+ is programmed to identify the chemical automatically, as is the case for nerve agent GB, nerve agent VX, and WP smoke, the circles are continuous. If a chemical is not automatically identified by PINS+, the circle is dashed, like nerve agent GA. Fill chemical types are identified in each decision-tree branch using the colors shown in Figure 4-3.

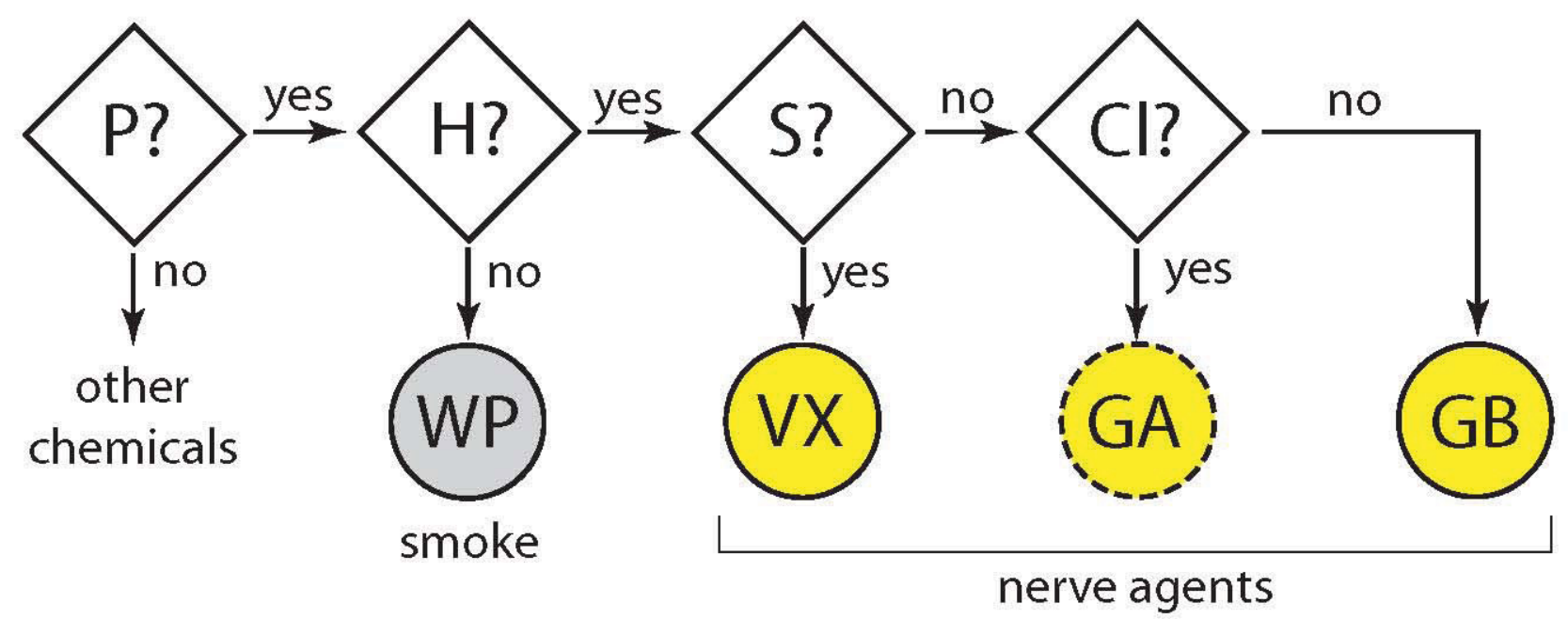

Figure 4-2: PINS phosphorus-based chemicals decision-tree branch.
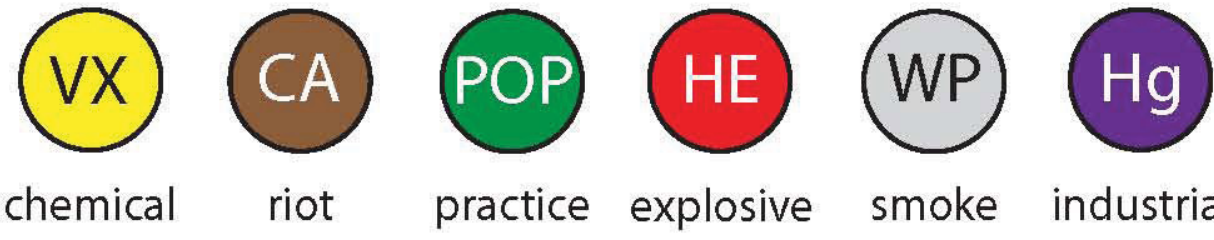

riot warfare agent practice

fill

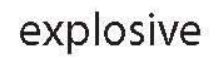

smoke industrial chemical agent

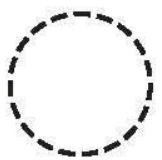

chemical not autoidentified by PINS+

Figure 4-3: Fill-chemical type color key. 


\section{Spectrum Identification Sheets}

Each chemical is described by a one- or two-page Spectrum Identification Sheet. The first page of each ID sheet describes the chemical and its spectral identification features, and an example for mustard agent is shown in Figure 4-4.
For those chemicals that might be confused with a related chemical substance, a second page contrasts the spectral features used to distinguish them. An example comparison page for mustard agent and FS smoke is shown in Figure 4-5.

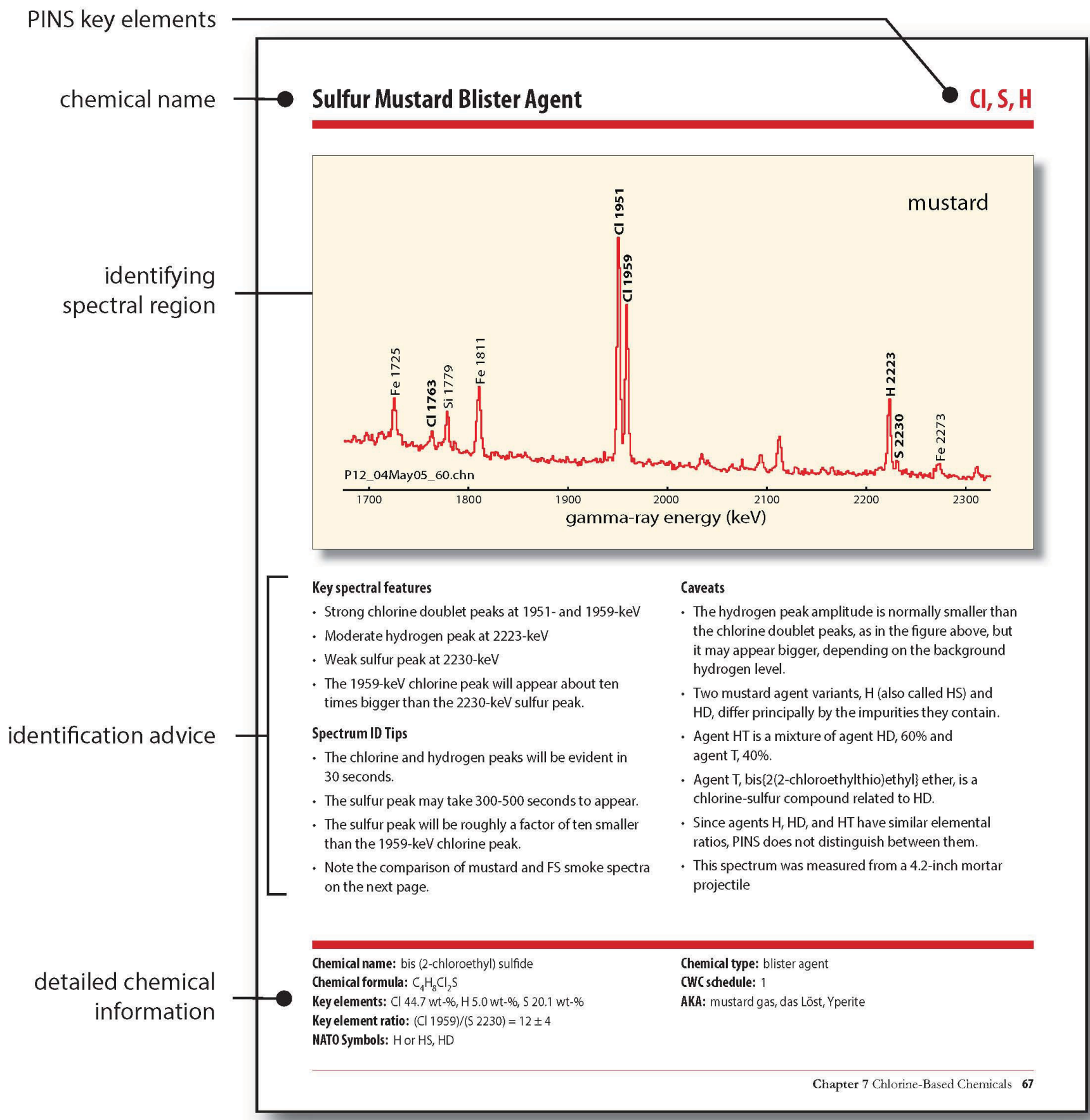

Figure 4-4: Spectrum ID Sheet for Sulfur Mustard Agent, Page 1. 
The y axes have been omitted in the spectrum ID sheets, because the heights of the gamma-ray peaks vary with assay geometry, californium-252 source strength, counting time, container-wall thickness, and detector efficiency. Instead, the peaks are characterized by intensity, that is, counts per 1000 seconds, using the descriptive scale of Table 4-1. Please note that in the multi-region spectral graphs, the $y$-axis scale may vary by region, for clarity.
Table 4-1 Gamma-Ray Peak Intensity Scale

\begin{tabular}{lc}
\hline Description & Intensity, counts/1,000 seconds \\
\hline very strong & $>10,000$ \\
strong & $>1,000$ \\
moderate & $>100$ \\
weak & $>10$ \\
very weak & $>1$ \\
\hline
\end{tabular}

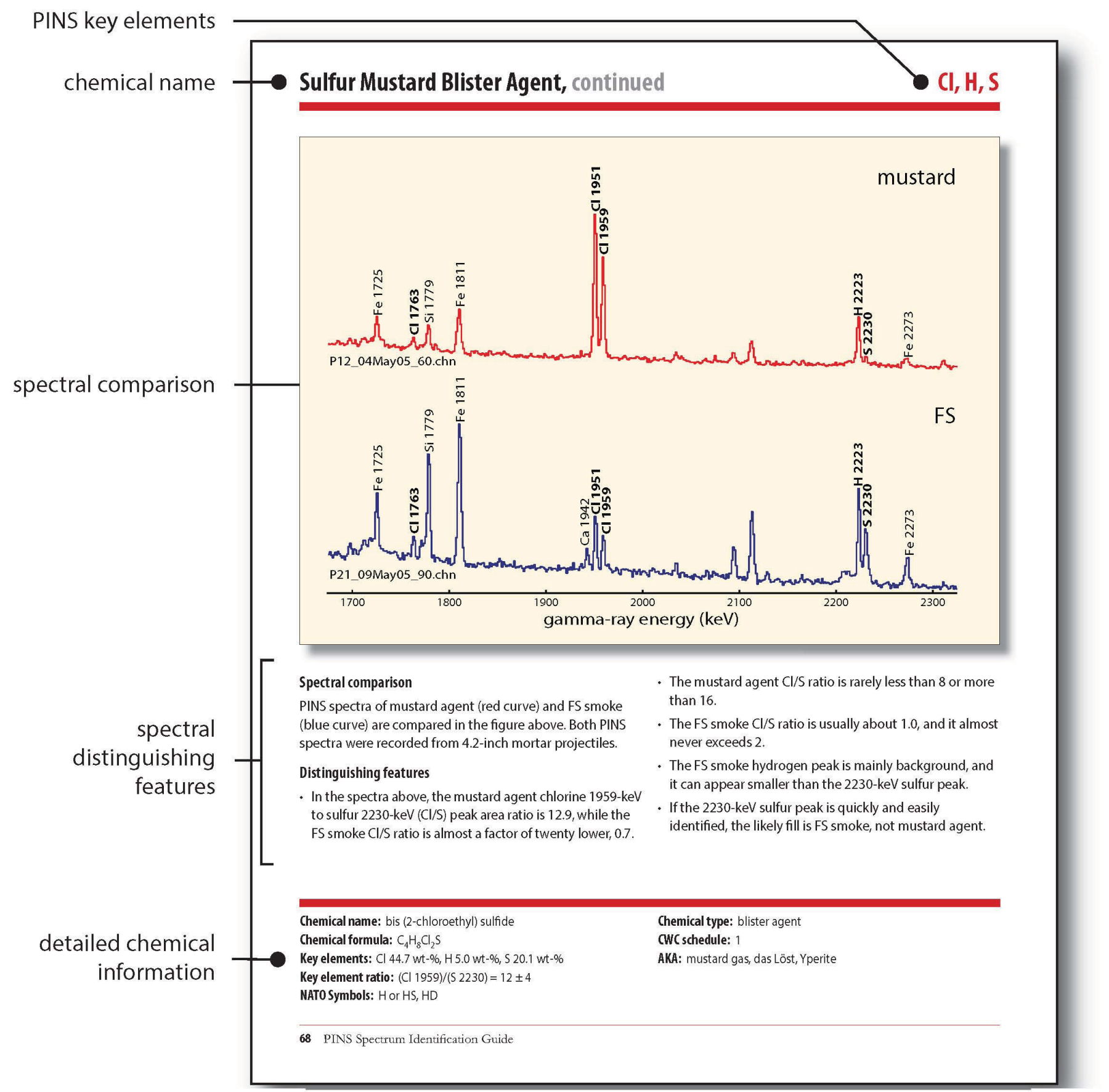

Figure 4-5: Spectrum ID Sheet for Sulfur Mustard Agent, Page 2. 


\section{Chapter 5}

\section{Phosphorus- Based Chemicals}

\section{Nerve agents}

All nerve agents are organophosphorus compounds,[1] and PINS can readily detect the presence of phosphorus in chemical warfare munitions filled with these chemicals. PINS has been used routinely to identify nerve agents GB and VX, and it identifies both automatically. Another nerve agent, GA, discussed below, soon will be added to the list of chemicals identified by PINS+.

\section{White Phosphorus}

White phosphorus (WP) is a common fill of conventional munitions, and is used for incendiary and screening-smoke effects. Chemically, it is $100 \%$ phosphorus, and it is one of the chemicals that is easily and quickly identified by PINS

\section{Nerve Agent GA}

Occasionally PINS has assessed German projectiles filled with nerve agent GA. [2] The GA key elements are similar to those in GB, except that GA contains 17.3 weight-\% nitrogen, and GB contains no nitrogen. However, all of the GA projectiles examined with PINS feature strong chlorine peaks in their spectra, from chlorobenzene added to the agent as a stabilizer. The chlorobenzene concentration in GA manufactured during World War II in Germany is said to be 5-20 weight-\%. [3]

\section{Spectral Identifications}

The algorithm for phosphorus-based chemical identification is shown in Figure 5-1. Spectra with phosphorus only, that is, little or no hydrogen, are produced by white phosphorusfilled munitions.

Nerve agent spectra are all characterized by strong hydrogen peaks, with the ratio of the $2223-\mathrm{keV}$ hydrogen peak net area to that of the $1266-\mathrm{keV}$ phosphorus peak net area exceeding 50 or more. Hence it is very easy to distinguish nerve agents from white phosphorus, based on $\mathrm{H} / \mathrm{P}$ ratios.

The individual nerve agents are identified by the presence or absence of other chemical elements. GB spectra feature strong hydrogen and weak phosphorus peaks. VX spectra include strong hydrogen and weak phosphorus peaks, plus a moderate sulfur peak at $5420 \mathrm{keV}$. GA spectra are similar to GB spectra, except that they include strong chlorine peaks at 1165,1951 , and $1959 \mathrm{keV}$.

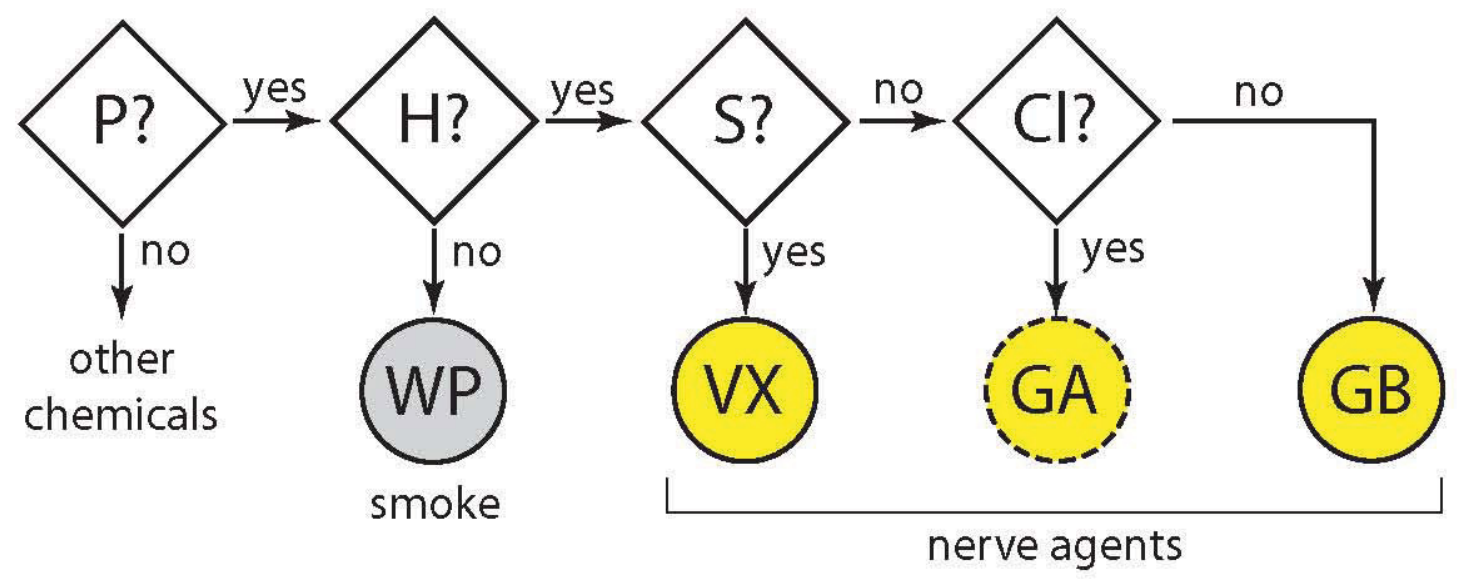

Figure 5-1. Phosphorus-based chemicals branch of the PINS decision tree. 


\section{References}

1. J.A.F.Com pton, Military Chemical and Biological Agents (Caldwell, N.J.: The Telford Press, 1987) pages 135-

174. Potential Military Chemical/Biological Agents and Compounds, U.S. Army Field Manual 3-11.9 (2005), pages II-13 to II-30.

2. The NATO two-letter designations for the G-series of nerve agents were established in October 1945 by Col. J.H. Rothschild of the U.S. Army Chemical Warfare Service. The newly-discovered German nerve agents were code-named as follows: Tabun-GA, Sarin - GB, Soman - GD, Ethylsarin - GE, Cyclosarin -GF, and Isopentylsarin - GH. There is no agent GC, to avoid possible confusion with phosgene, agent CG.

For more details, see the recent text by Jonathan B. Tucker, War of Nerves (New York: Pantheon Books, 2006) pages 105-6.

3. Private communication, Lucille Forrest, Office of the U.S. Army Project Manager for Non-Stockpile Chemical Materiel, 3 February 2012. 


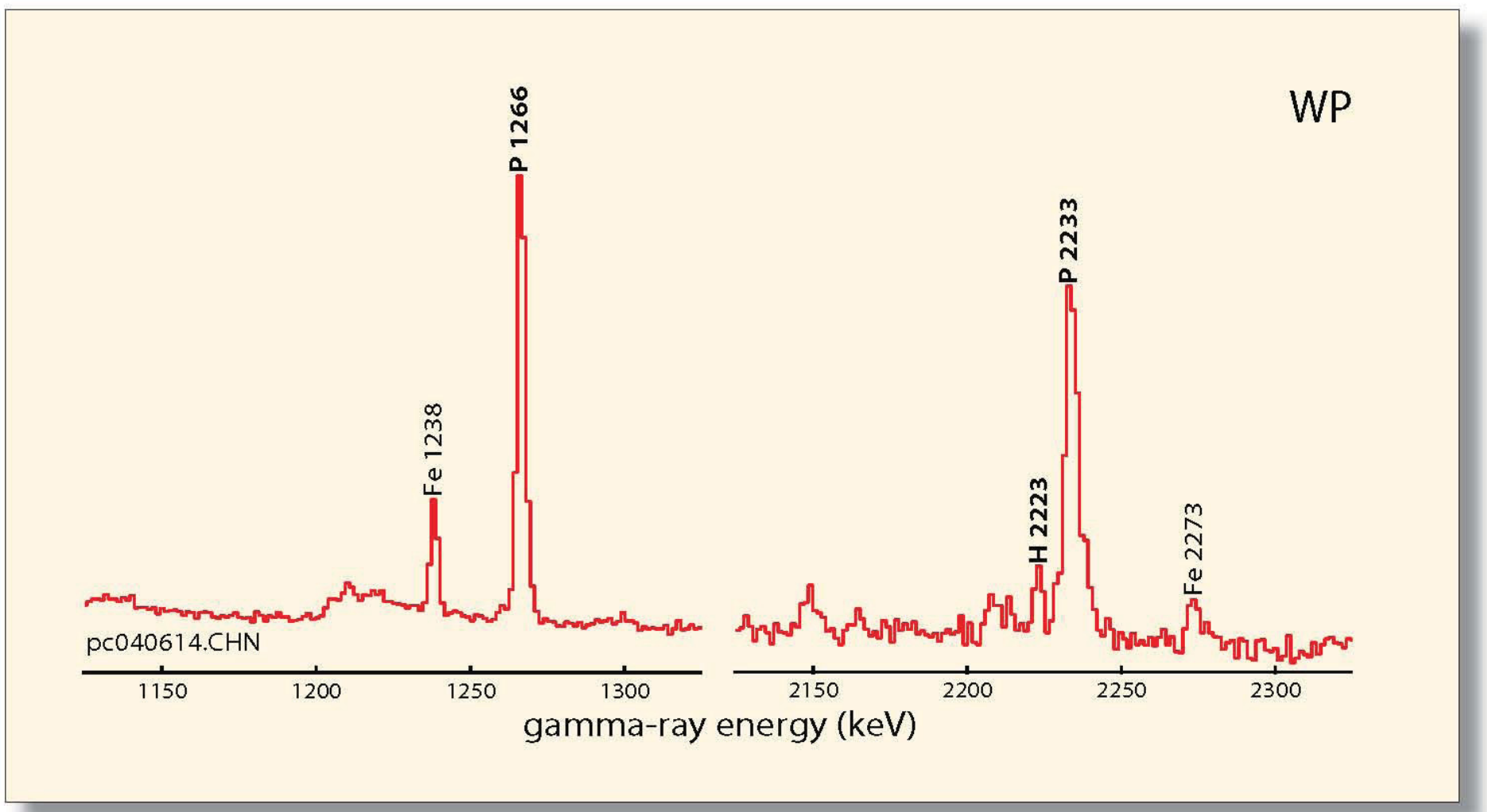

\section{Key spectral features}

- Strong phosphorus peak at $1266 \mathrm{keV}$

- Moderate phosphorus peak at $2233 \mathrm{keV}$

- Weak or moderate hydrogen peak at $2223 \mathrm{keV}$

\section{Spectrum ID Tips}

- The 1266-keV phosphorus peak is very strong, and normally it can be seen in 30 seconds, to the right of the 1238-keV iron peak, and it may even obscure the small 1261-keV iron peak.

- The 2233-keV phosphorus peak, just to the right of the 2223-keV hydrogen peak, is always seen in WP spectra. If this phosphorus peak is not observed, the fill is likely a nerve agent.

\section{Caveats}

- The true WP hydrogen-to-phosphorus ratio is, of course, zero. The measured $\mathrm{H} / \mathrm{P}$ ratio may be non-zero, due to incomplete hydrogen background subtraction, but it should be less than 0.25 .

- If the background is high, the hydrogen peak may appear bigger than the 2233-keV phosphorus peak.

- This spectrum was measured from a 4.2 inch mortar projectile.
Chemical names: white phosphorus

Chemical formula: $P_{4}$

Key elements: phosphorus, $100 \mathrm{wt}-\%$

Key element ratio: $(H$ 2223)/(P 1266) < 0.25
NATO Symbol: WP

Chemical type: incendiary, screening smoke

CWC schedule: $n / a$ 


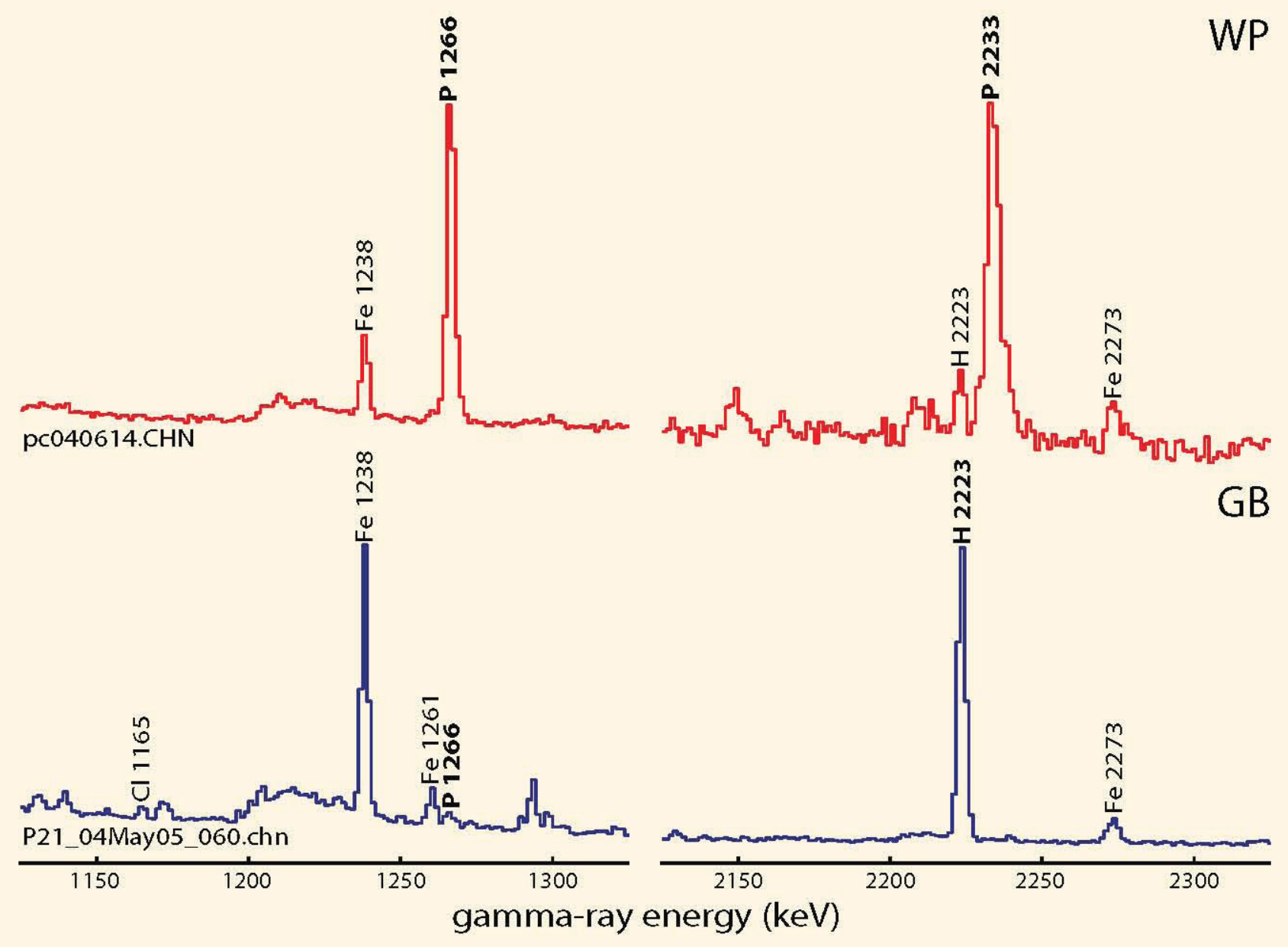

\section{Spectral comparison}

The phosphorus and hydrogen spectral regions of GB nerve agent (blue curve) and white phosphorus (red) are compared in the figure above. The GB PINS spectrum was measured from a $155-\mathrm{mm}$ artillery projectile, and the WP spectrum from a 4.2-inch mortar projectile.

\section{Distinguishing features}

- A weak-to-moderate phosphorus peak is observed in the $\mathrm{GB}$ spectrum at $1266 \mathrm{keV}$, while the WP peak at this energy is strong.
- No phosphorus peak is observed at $2233 \mathrm{keV}$ in the GB spectrum, but a moderate phosphorus peak is observed in the WP spectrum.

- A strong hydrogen peak is seen in the $G B$ spectrum at $2223 \mathrm{keV}$.

- The hydrogen peak in a WP spectrum is weak-tomoderate, and it is entirely due to background.

- If the 2233-keV phosphorus peak is quickly and easily identified, the likely fill is WP smoke, not a nerve agent.
Chemical names: white phosphorus

Chemical formula: $P_{4}$

Key elements: phosphorus, $100 \mathrm{wt}-\%$

Key element ratio: $(\mathrm{H} 2223) /(\mathrm{P} 1266)<0.25$
NATO Symbol: WP

Chemical type: incendiary, screening smoke

CWC schedule: $n / a$ 


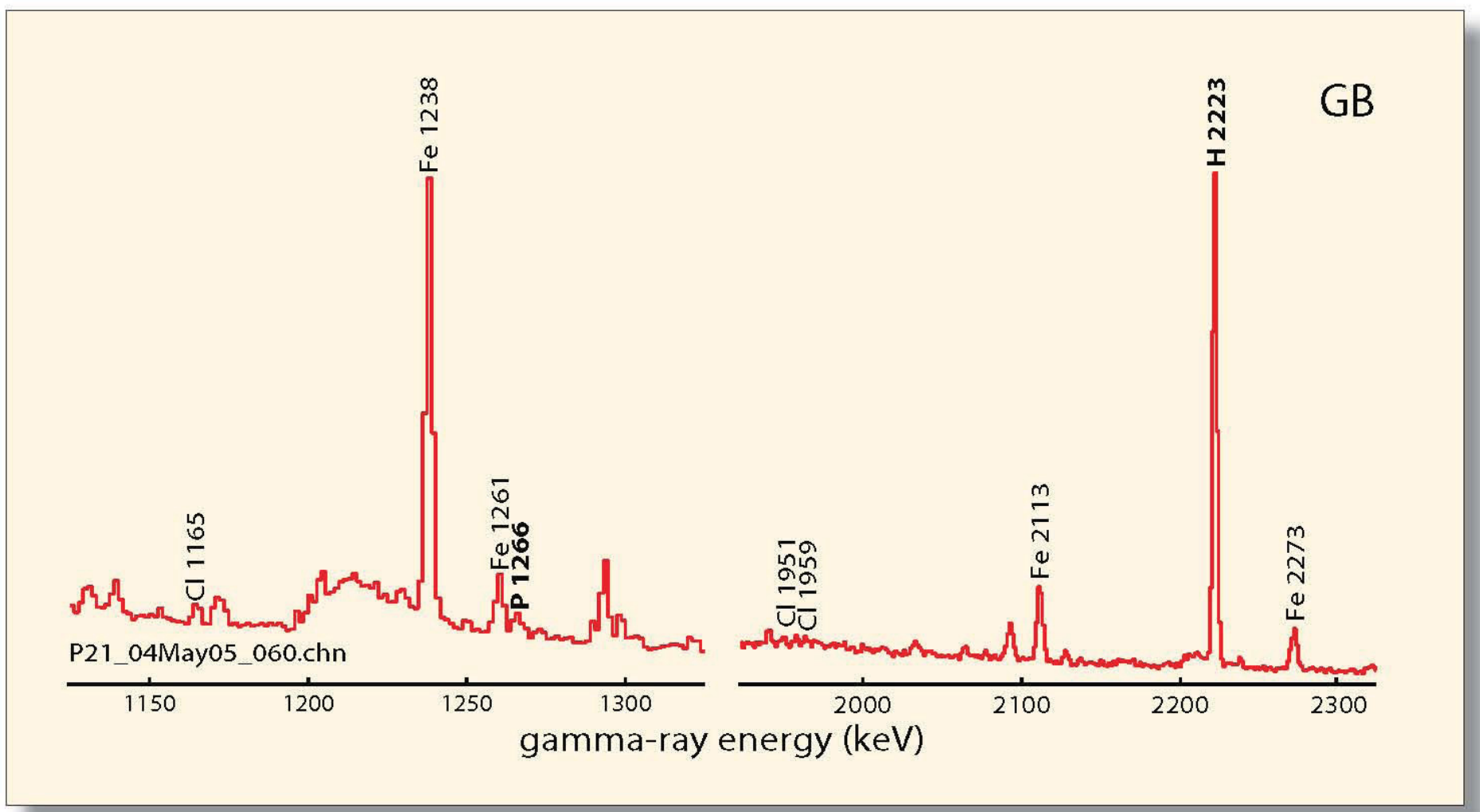

\section{Key spectral features}

- Moderate phosphorus peak at $1266 \mathrm{keV}$

- Strong hydrogen peak at $2223 \mathrm{keV}$

\section{Spectrum ID Tips}

- The 1266-keV phosphorus peak is not very strong, but normally it will be seen in 100-300 seconds, to the immediate right of the 1261-keV iron peak.

- The hydrogen peak is at least a factor of four stronger than the 1266-keV phosphorus peak.

- In spectra from steel containers and projectiles, the 1266$\mathrm{keV}$ phosphorus peak is about a factor of five smaller than the nearby 1238-keV iron peak.

\section{Caveats}

- GB contains no sulfur. If sulfur is detected in addition to phosphorus and hydrogen, the fill is VX.

- The 2233-keV phosphorus peak is rarely observed in GB spectra.

- If a moderate or strong peak is evident at $2233 \mathrm{keV}$, the likely fill is WP smoke.

- Weak chlorine peaks at 1951 and 1959 may be seen in GB spectra due to impurities.

- Chlorine peaks, in GB, if any, will be a factor of 50 smaller than the hydrogen peak.

- This spectrum was measured from a 155-mm artillery projectile.
Chemical name: isopropyl methylphosphonofluoridate

Chemical formula: $\mathrm{C}_{4} \mathrm{H}_{10} \mathrm{FO}_{2} \mathrm{P}$

Key elements: phosphorus, 22 wt- $\%$, hydrogen 7.1 wt- $\%$

Key element ratio: $(\mathrm{H} 2223) /(\mathrm{P} 1266)>20$
NATO Symbol: GB

Chemical type: nerve agent

CWC schedule: 1

AKA: sarin 


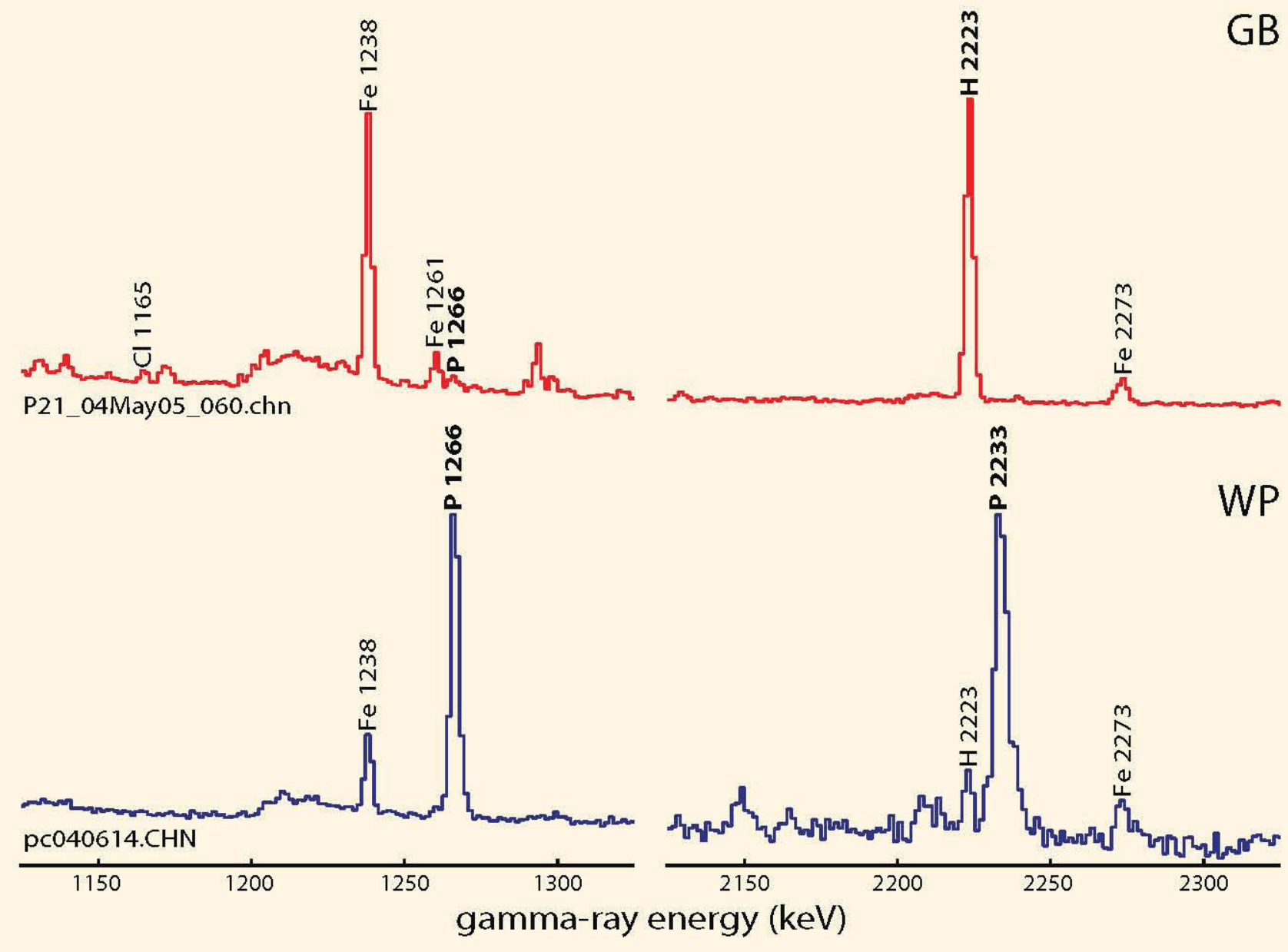

\section{Spectral comparison}

The phosphorus and hydrogen spectral regions of $\mathrm{GB}$ nerve agent (red curve) and white phosphorus (blue) are compared in the figure above. The GB PINS spectrum was measured from a $155-\mathrm{mm}$ artillery projectile, and the WP spectrum from a 4.2-inch mortar projectile.

\section{Distinguishing features}

- A weak-to-moderate phosphorus peak is observed in the GB spectrum at $1266 \mathrm{keV}$, while the WP peak at this energy is strong.
- No phosphorus peak is observed at $2233 \mathrm{keV}$ in the GB spectrum, but a moderate phosphorus peak is observed in the WP spectrum.

- A strong hydrogen peak is seen in the GB spectrum at $2223 \mathrm{keV}$.

- The hydrogen peak in a WP spectrum is weak-tomoderate, and it is entirely due to background.

- If the 2233-keV phosphorus peak is quickly and easily identified, the likely fill is WP smoke, not a nerve agent.
Chemical name: isopropyl methylphosphonofluoridate

Chemical formula: $\mathrm{C}_{4} \mathrm{H}_{10} \mathrm{FO}_{2} \mathrm{P}$

Key elements: phosphorus, 22 wt- $\%$, hydrogen 7.1 wt- $\%$

Key element ratio: $(H$ 2223)/(P 1266) $>20$
NATO Symbol: GB

Chemical type: nerve agent

CWC schedule: 1

AKA: sarin 


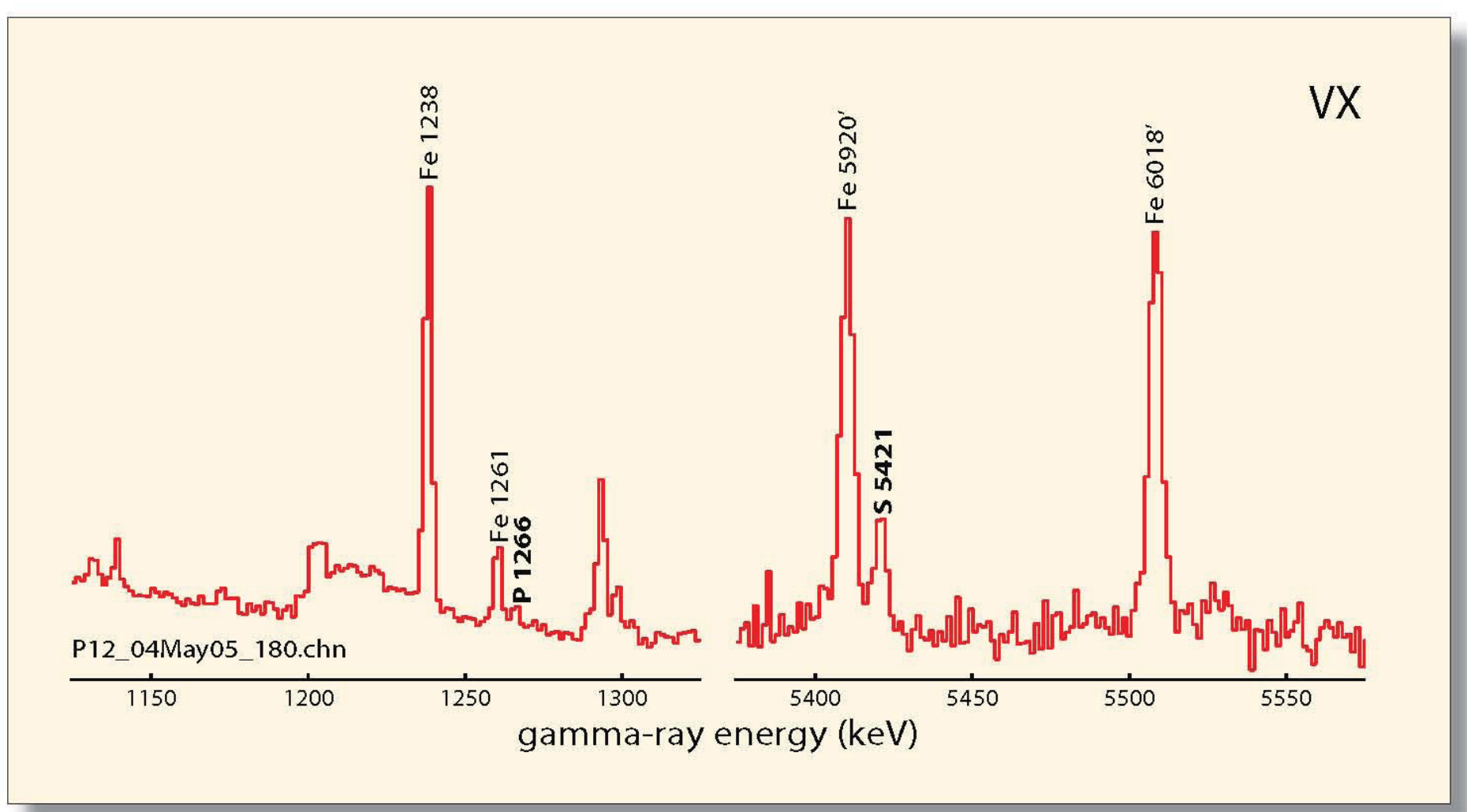

\section{Key spectral features}

- Weak or moderate phosphorus peak at $1266 \mathrm{keV}$

- Strong or very strong hydrogen peak at $2223 \mathrm{keV}$

- Moderate sulfur peak at $5420 \mathrm{keV}$

- The hydrogen/phosphorus peak ratio will be 50 or greater.

\section{Spectrum ID Tips}

- The $5420-k e V$ sulfur peak will be observed before the 1266-keV phosphorus peak.

- The VX 1266-keV phosphorus peak is not very strong, and normally it will be seen in $200-600$ seconds, to the immediate right of the 1261-keV iron peak.

- The 2230-keV sulfur peak and the 2233-keV phosphorus peak are not observed in VX spectra.

\section{Caveats}

- If the detector resolution is $<80 \%$, it may take over 1,000 seconds to observe the VX 1266-keV phosphorus peak.

- This spectrum was measured from a 155-mm artillery projectile.
Chemical name : O-ethyl-S-(2-diisopropylaminoethyl) methyl phosphonothiolate

Chemical formula: $\mathrm{C}_{11} \mathrm{H}_{26} \mathrm{NO}_{2} \mathrm{PS}$

Key elements: P 11.6 wt- $\%$, hydrogen 9.7 wt- $\%$, S 12.0 wt- $\%$

Key element ratios: $(\mathrm{H} 2223) /(\mathrm{P} 1266)>50, \mathrm{P} / \mathrm{S}=1.1 \pm 0.7$
NATO Symbol: $V X$

Chemical type: nerve agent

CWC schedule: 1 

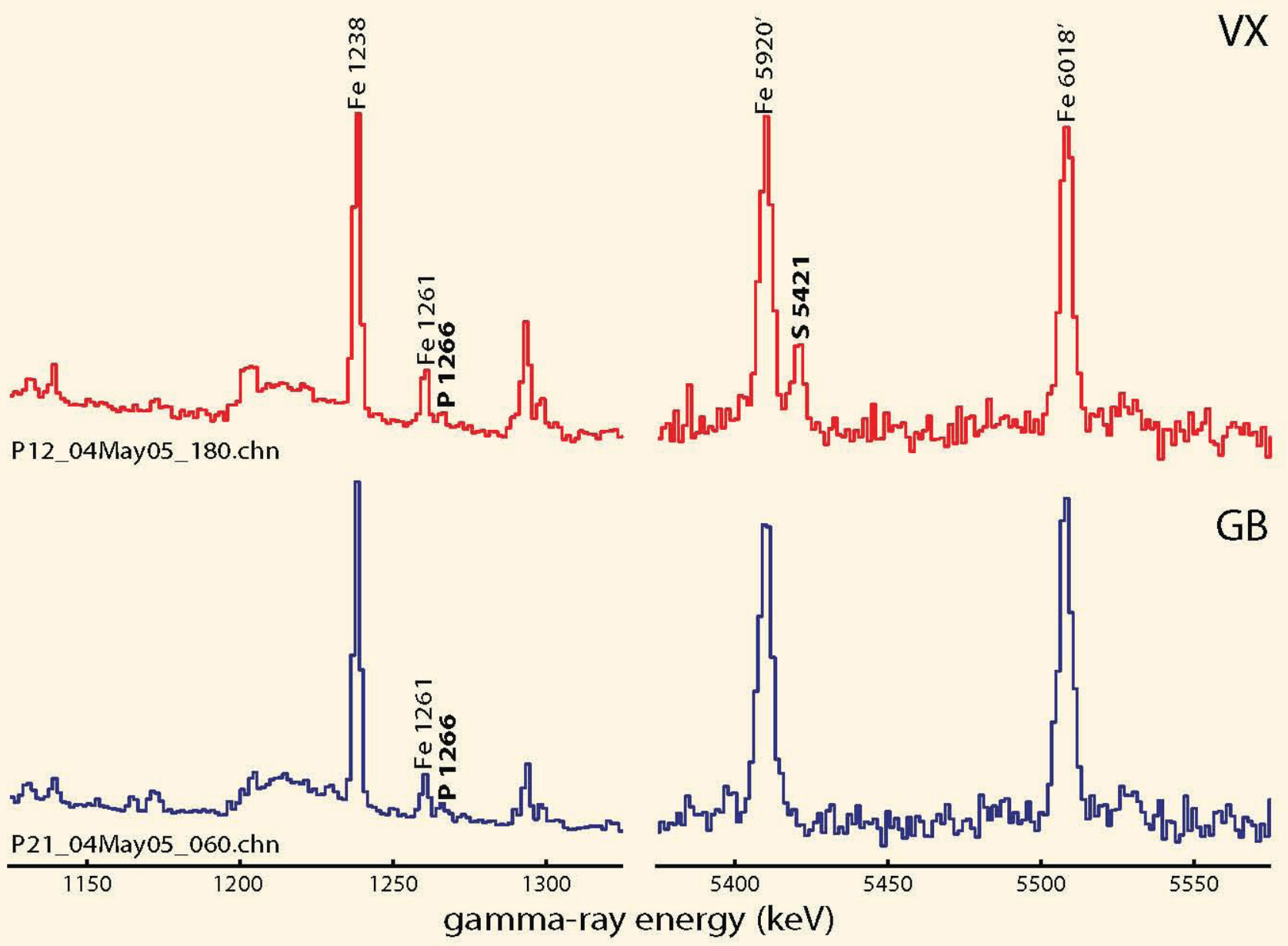

\section{Spectral comparison}

The phosphorus and sulfur spectral regions of VX (red curve) and GB (blue) nerve agents are compared in the figure above. Both PINS spectra were measured from 155$\mathrm{mm}$ artillery projectiles.

\section{Distinguishing features}

- The sulfur peak at $5420 \mathrm{keV}$ distinguishes VX from GB. $\mathrm{GB}$ contains no sulfur.
- Both agents have a weak-to-moderate 1266-keV phosphorus peak in their spectra.

- The VX phosphorus peak is usually weaker than the GB phosphorus peak, and it takes longer to identify.
Chemical name: 0-ethyl-S-(2-diisopropylaminoethyl) methyl phosphonothiolate

Chemical formula: $\mathrm{C}_{11} \mathrm{H}_{26} \mathrm{NO}_{2} \mathrm{PS}$

Key elements: P 11.6 wt-\%, hydrogen 9.7 wt- $\%$, S 12.0 wt- $\%$

Key element ratios: $(H 2223) /(\mathrm{P} 1266)>50, P / S=1.1 \pm 0.7$
NATO Symbol: $V X$

Chemical type: nerve agent

CWC schedule: 1 


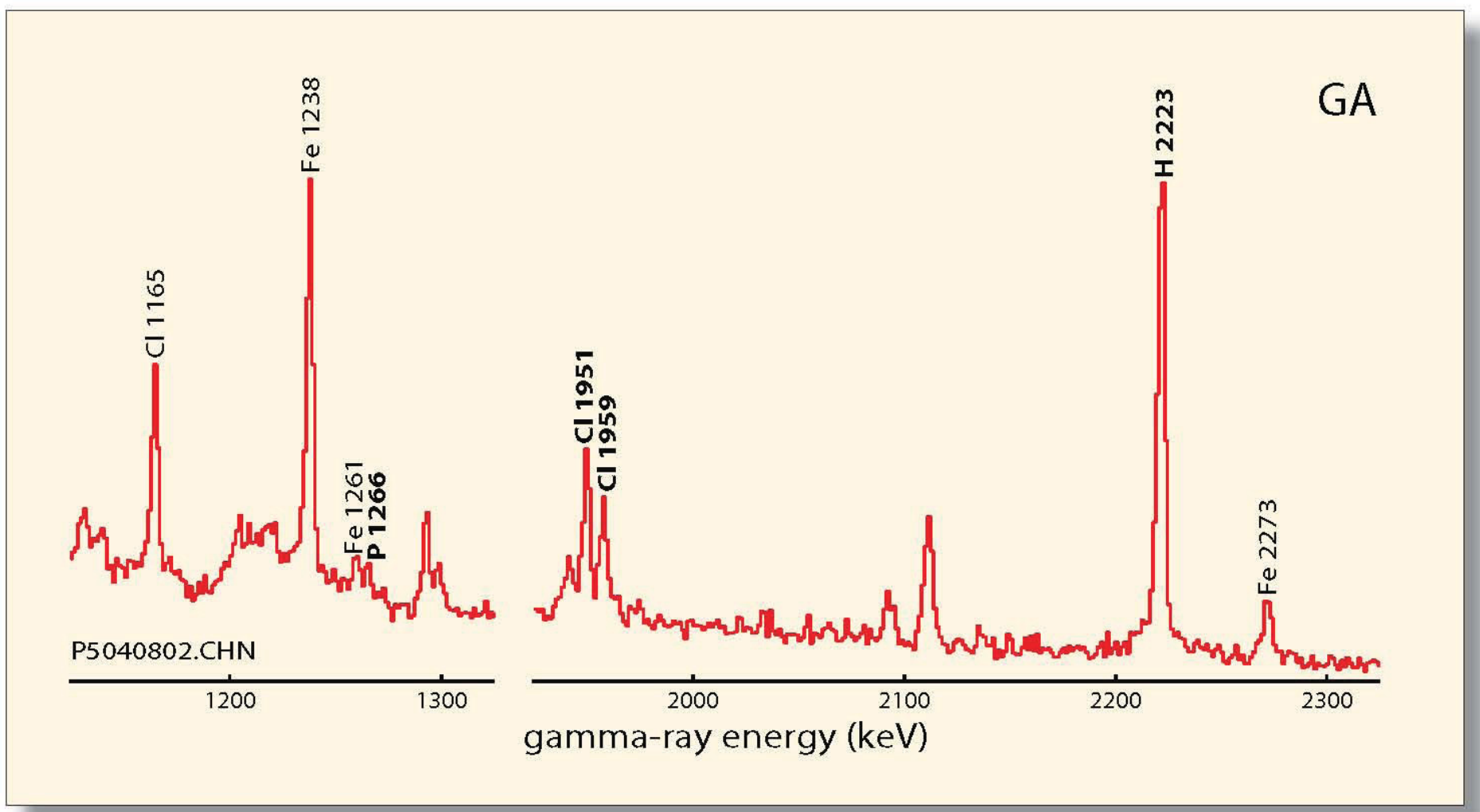

\section{Key spectral features}

- Moderate phosphorus peak at $1266 \mathrm{keV}$

- Strong chlorine peaks at 1951 and $1959 \mathrm{keV}$

- Strong hydrogen peak at $2223 \mathrm{keV}$

- Weak nitrogen peaks at 10318 and $10829 \mathrm{keV}$

\section{Spectrum ID Tips}

- The chlorine and hydrogen peaks will appear in 30 seconds.

- The 1266-keV phosphorus peak will appear in 200-300 seconds.

- It may take over 2,000 seconds to observe one of the nitrogen peaks.

- The 2233-keV P peak, just to the right of the 2223-keV H peak, is rarely observed in GA spectra.

\section{Caveats}

- Pure GA contains no chlorine - it must be distinguished from $\mathrm{GB}$ by detection of nitrogen.

- During WW II, Germany added 20 wt-\% chlorobenzene to $G A$ as a stabilizer.

- This spectrum was measured from a World War II-era German 105-mm artillery projectile.

- PINS spectra have been measured from less than five GA-filled projectiles.
Chemical name: ethyl N, N-dimethylphosphoroamidocyanidate Chemical formula: $\mathrm{C}_{5} \mathrm{H}_{11} \mathrm{~N}_{2} \mathrm{O}_{2} \mathrm{P}$

Key elements: P 19.1 wt-\%, H 6.8 wt- $\%$, N 17.3 wt- $\%, C l 6.2$ wt- $\%$ Key element ratios: $(\mathrm{H} 2223) /(\mathrm{P} 1266)=11.2 \pm 2.1$

$(\mathrm{Cl} 1959) /(\mathrm{P} \mathrm{1266})=2.6 \pm 0.25$
NATO Symbol: GA

Chemical type: nerve agent

CWC schedule: 1

AKA: tabun 


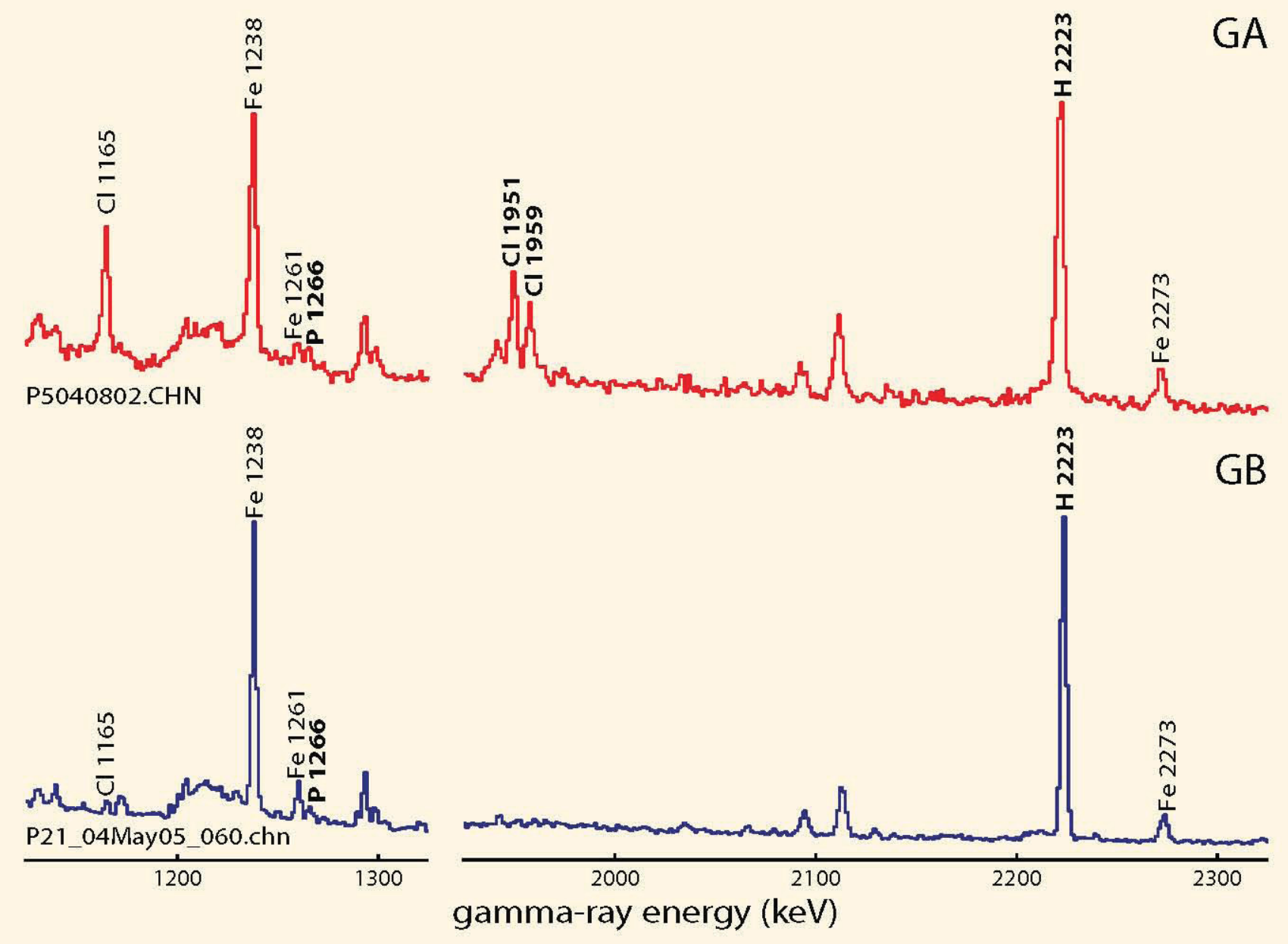

\section{Spectral comparison}

The phosphorus and hydrogen spectral regions of GA nerve agent (red curve) and GB nerve agent (blue) are compared in the figure above. The GA PINS spectrum was measured from a $105-\mathrm{mm}$ projectile, and the GB spectrum from a $155-\mathrm{mm}$ artillery projectile.

\section{Distinguishing features}

- Both agents produce weak-to-moderate phosphorus peaks at $1266 \mathrm{keV}$.
- A strong hydrogen peak is seen in both $G A$ and $G B$ spectra at $2223 \mathrm{keV}$.

- Strong chlorine peaks at 1951 and $1959 \mathrm{keV}$ are observed in GA spectra, from the chlorobenzene stabilizer added to this agent.

- Chlorine peaks from an impurity are weak or absent in GB spectra.
Chemical name: ethyl N, N-dimethylphosphoroamidocyanidate

Chemical formula: $\mathrm{C}_{5} \mathrm{H}_{11} \mathrm{~N}_{2} \mathrm{O}_{2} \mathrm{P}$

Key elements: P 19.1 wt- $\%, \mathrm{H} 6.8$ wt-\%, N 17.3 wt- $\%, \mathrm{Cl} 6.2$ wt-\%

Key element ratios: $(\mathrm{H} 2223) /(\mathrm{P} 1266)=11.2 \pm 2.1$

$(\mathrm{Cl} 1959) /(\mathrm{P} 1266)=2.6 \pm 0.25$
Chemical type: nerve agent CWC schedule: 1

AKA: tabun

NATO Symbol: GA 


\section{Chapter 6}

\section{Arsenicals}

A Il arsenical chemicals are poisonous substances.[1] The arsenical compound frequently mentioned in detective stories is arsenic trioxide. In this chapter, the discussion will be confined to five arsenical compounds of potential use in chemical warfare.

\section{Blood Agent Arsine}

Arsine is World War I-era blood agent,[2] and this toxic compressed gas destroys red blood cells. Arsine has found present-day use in the semiconductor industry. Unlike the other arsenical compounds of this chapter, arsine- $\mathrm{AsH}_{3}-$ contains no chlorine.

\section{Arsenic Trichloride}

Arsenic trichloride, $\mathrm{AsCl}_{3}$, is an industrial chemical,[3] and a chemical warfare agent precursor.[4]

\section{Arsenical Blister Agents}

Lewisite is a blister agent developed during World War I. [5]

Agent Blue Ring-2 was manufactured in Germany during World War II, and its principal components are blister agent phenyldichloroarsine and vomiting agent diphenyldichloroarsine.[6]

Winterlöst (WL) or winter mustard was also manufactured in Germany during World War II, by adding $40-50 \%$ phenyldichloroarsine blister agent to $60-50 \%$ mustard blister agent. The addition of an arsenical to mustard can lower its freezing point from $14^{\circ} \mathrm{C}\left(57^{\circ} \mathrm{F}\right)$ to below $-25^{\circ} \mathrm{C}\left(-13^{\circ} \mathrm{F}\right)$. [7]

\section{Spectral Identifications}

The algorithm for distinguishing between these arsenical compounds is shown in Figure 6-1. Spectra with arsenic and hydrogen only are produced by munitions containing arsine, agent SA.

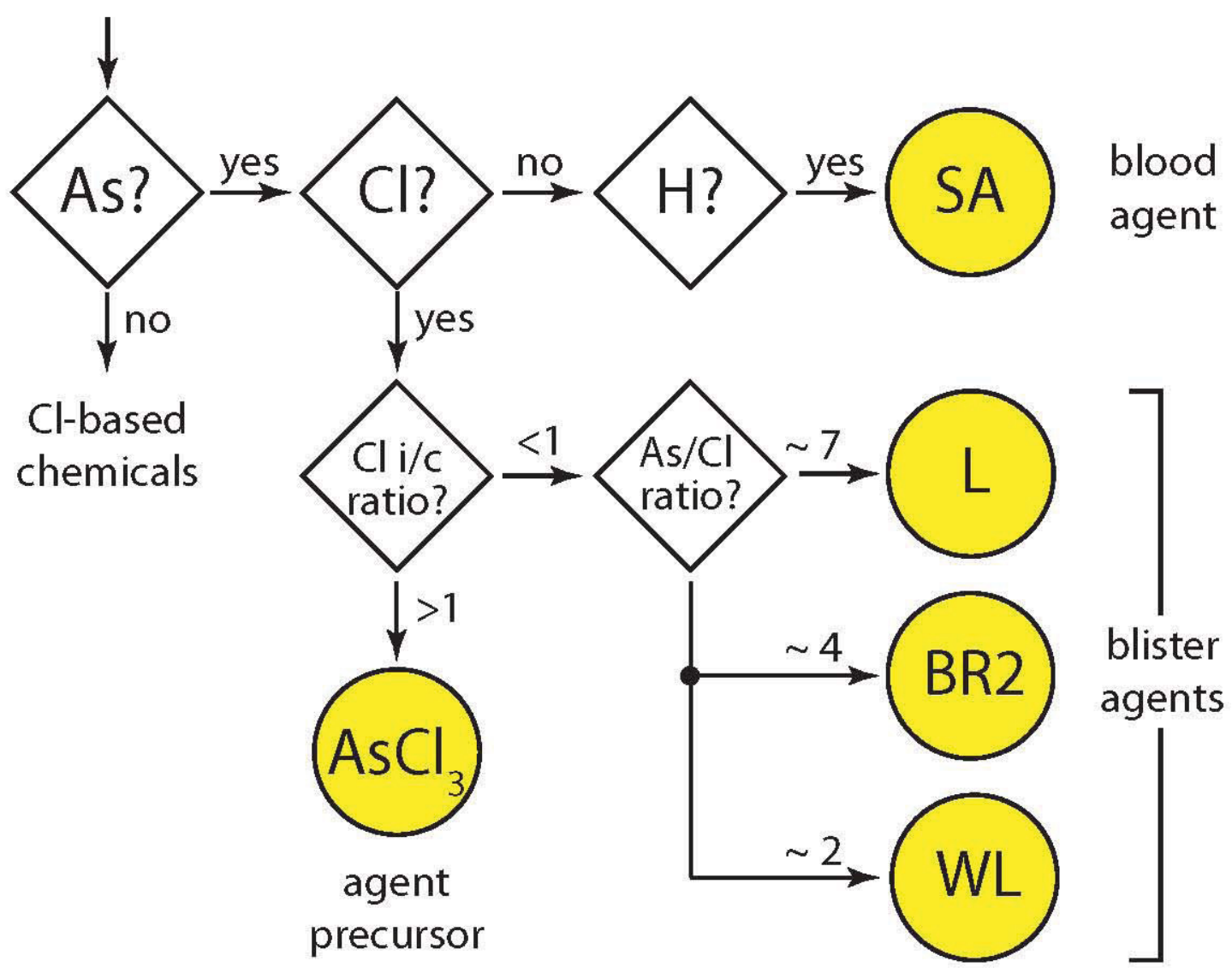

Figure 6-1: Arsenical branch of the PINS decision tree. 
The other four arsenicals contain chlorine. Arsenic trichloride contains no hydrogen, and hence the ratio of its $1763-\mathrm{keV}$ chlorine inelastic peak to 1959 chlorine capture peak should be 1.0 or greater. This ratio is abbreviated "Cl i/c ratio" in the Figure. The other three arsenicals contain hydrogen, and their chlorine inelastic-to-capture ratios should all be less than one.

Lewisite (L) can be recognized by a high ratio, about 7, of the $279-\mathrm{keV}$ arsenic inelastic peak to the $1959-\mathrm{keV}$ chlorine capture peak. The As $/ \mathrm{Cl}$ peak ratio is approximately 3.5 for agent blue ring 2 (BR2), and the ratio is about 1.7 for winter mustard. Also, a weak sulfur peak is observed at $2231 \mathrm{keV}$ in winter mustard spectra.

\section{References}

1 M.J. O'Neil, ed. The Merck Index, 13th Edition (Whitehouse Station, NJ: Merck \& Co., Inc., 2001) article 802 .

2. Potential Military Chemical/Biological Agents and Compounds, U.S. Army Field Manual (FM) 3-11.9 (2005) pages II-35 to II-37.

3. John Emsley, Nature's Building Blocks: An A-Z Guide to the Elements (Oxford, United Kingdom: Oxford University Press, 2001) page 18.

4. James A.F. Compton, Military Chemical and Biological Agents (Caldwell, NJ: The Telford Press, 1987) pages 19-39.

5. Compton, pages 37-38.

6. R. A. Zellermann and L. Holte, " $15 \mathrm{~cm}$ Traktor Rocket 41 DOV” Pegasus OHG Report F 99 - 181604, August, 1999. This report is not paginated, but the blue ring 2 chemical fill is described in section 2.6.

7. U.S. Army FM 3-11.9, pages II-38 and II-54. 


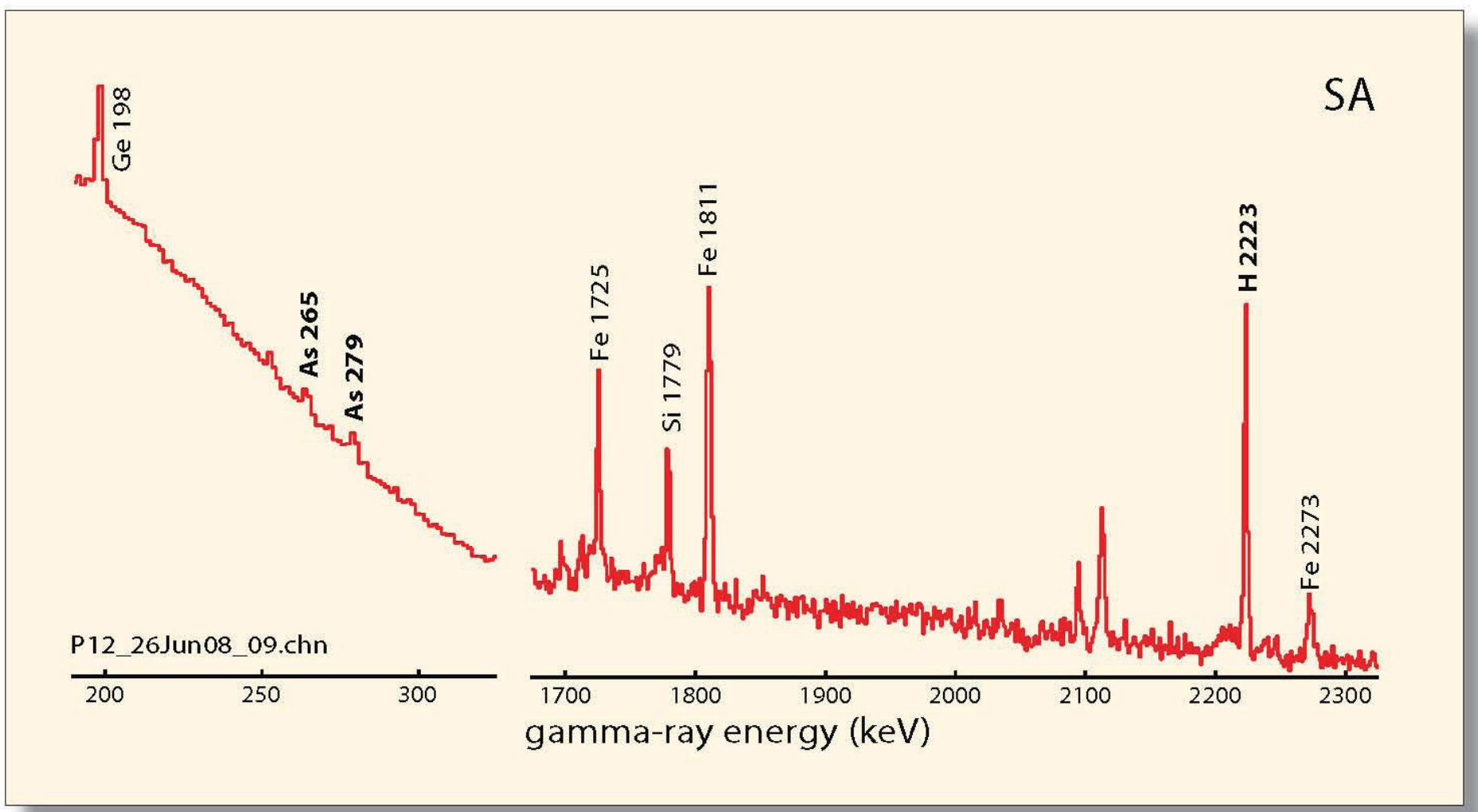

\section{Key spectral features}

- Moderate or strong arsenic peaks at 265 and $279 \mathrm{keV}$

- Moderate hydrogen peak at $2223 \mathrm{keV}$

\section{Spectrum ID Tips}

- Arsenic peaks are seen at 265 and $279 \mathrm{keV}$, above a slanting Compton continuum.

- The arsenic peaks may be subtle, especially with a small munition like the $75-\mathrm{mm}$ projectile assayed in the figure above.

- The PINS software may report zero hydrogen if the background is over-subtracted.

- If arsenic is detected, but chlorine peaks are not observed, classify the fill chemical as SA.

\section{Caveats}

- The (Cl 1959)/(H 2223) peak area ratio may vary, depending on background subtraction effects.

- This spectrum was measured from a 75-mm artillery projectile.

- PINS spectra have been measured from only a handful of SA-filled projectiles.
Chemical name: arsine

Chemical formula: $\mathrm{AsH}_{3}$

Key elements: arsenic 96.1 wt- $\%$, hydrogen 3.9 wt- $\%$

Key element ratio: (As 279) $/(\mathrm{H} 2223)=1.0 \pm 0.3$

NATO Symbol: SA
Chemical type: blood agent

CWC schedule: $n / a$

AKA: arsenic trihydride 


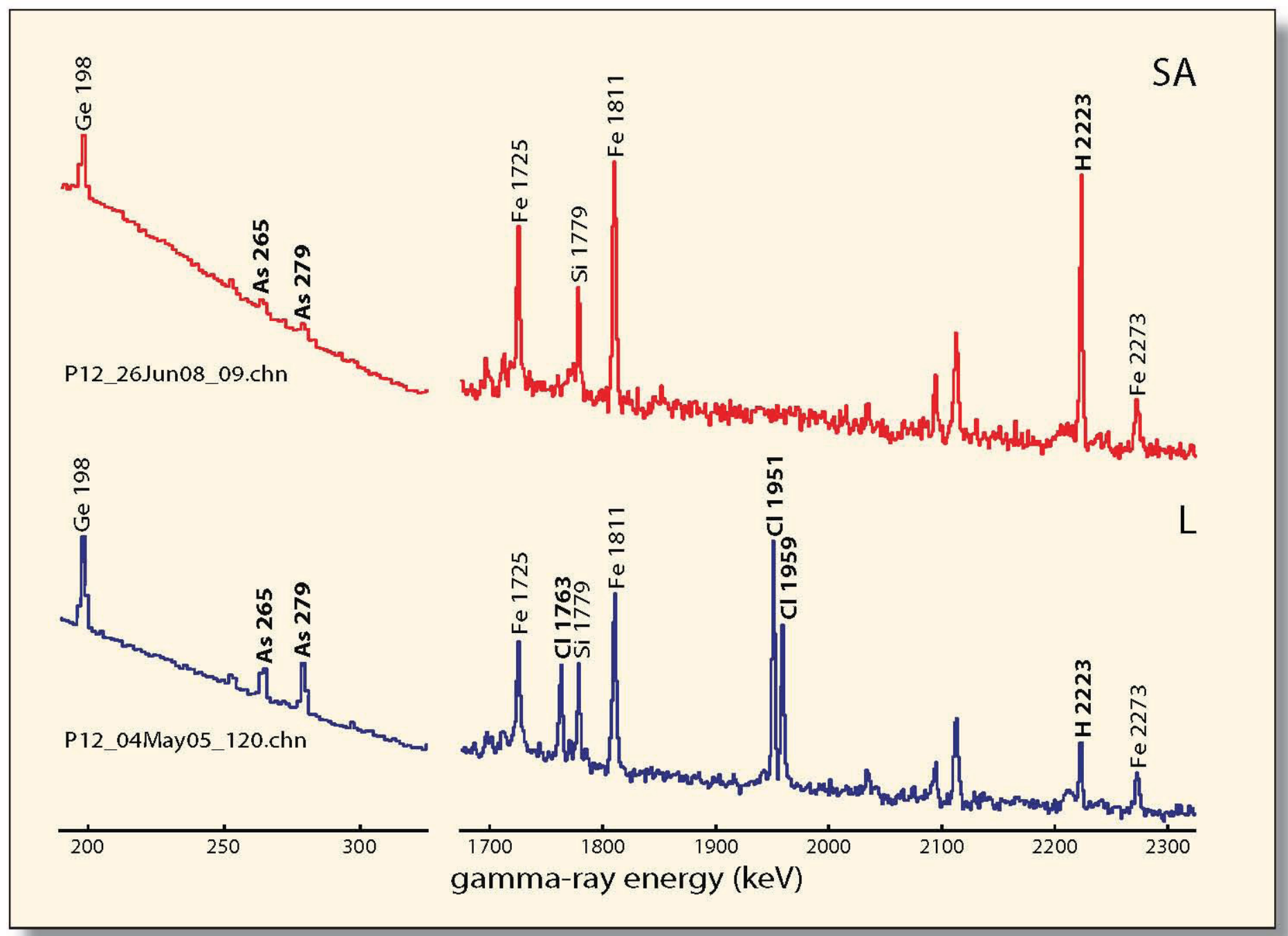

\section{Spectral comparison}

The arsenic and chlorine-hydrogen spectral regions of SA blood agent (red curve) and L blister agent (blue) are compared in the figure above. The SA PINS spectrum was measured from a $75-\mathrm{mm}$ artillery projectile, and the $\mathrm{L}$ spectrum from a 4.2-inch mortar projectile.

\section{Distinguishing features}

- Moderate-to-strong arsenic peaks are observed in both the $S A$ and $L$ spectra at 265 and $279 \mathrm{keV}$.

- Strong chlorine peaks are seen in the L spectrum at 1763 , 1951 , and $1959 \mathrm{keV}$.

- No chlorine peaks are observed in the SA spectrum.
Chemical name: arsine

Chemical formula: $\mathrm{AsH}_{3}$

Key elements: arsenic 96.1 wt-\%, hydrogen 3.9 wt-\%

Key element ratio: (As 279) $/(\mathrm{H} 2223)=1.0 \pm 0.3$

NATO Symbol: SA
Chemical type: blood agent

CWC schedule: $n / a$

AKA: arsenic trihydride 


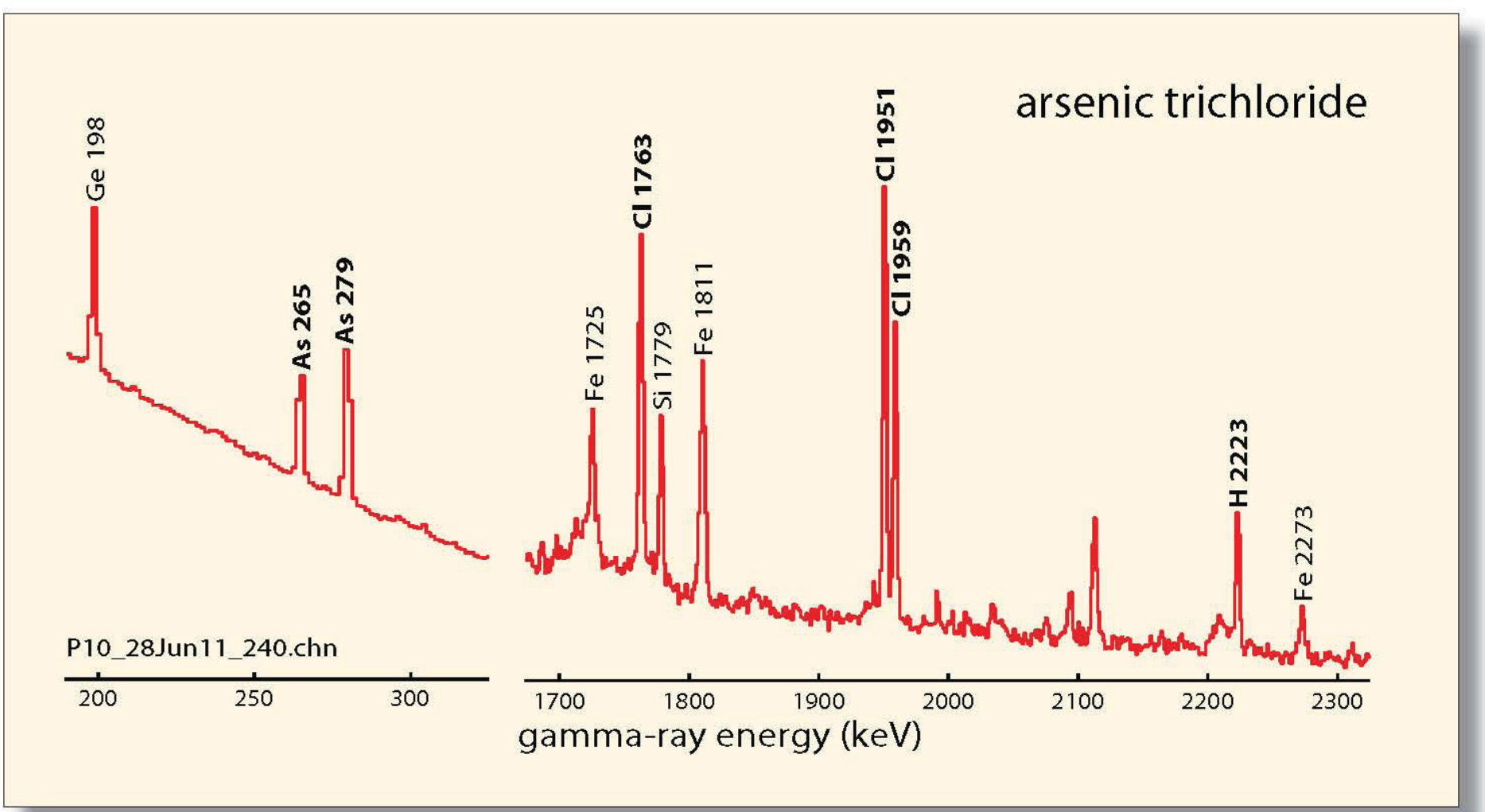

\section{Key spectral features}

- Strong or very strong As peaks at 265 and $279 \mathrm{keV}$

- Moderate or strong chlorine peaks at 1763, 1951, and $1959 \mathrm{keV}$

\section{Spectrum ID Tips}

- Arsenic peaks are seen at 265 and $279 \mathrm{keV}$, above a slanting Compton continuum.

- Arsenic trichloride contains no hydrogen, and its chlorine inelastic-to-capture (1763/1959) ratio is over 1.0

\section{Caveats}

- The key element ratios below were calculated after background subtraction.

- This spectrum was measured from a laboratory simulant.

- No PINS spectra have been measured from arsenic trichloride-filled containers or munitions.
Chemical name: arsenic trichloride

Chemical formula: $\mathrm{AsCl}_{3}$

Key elements: arsenic $41.3 w t-\%$, chlorine $58.7 w t-\%$

Key element ratios: $($ As 279) $/(\mathrm{Cl} 1959)=9.3 \pm 1.2$

$(\mathrm{Cl} 1763) /(\mathrm{Cl} 1959)=1.2 \pm 0.2$
NATO Symbol: $n / a$

Chemical type: agent precursor

CWC schedule: $2 \mathrm{~B}$

AKA: butter of arsenic

fuming liquid arsenic 


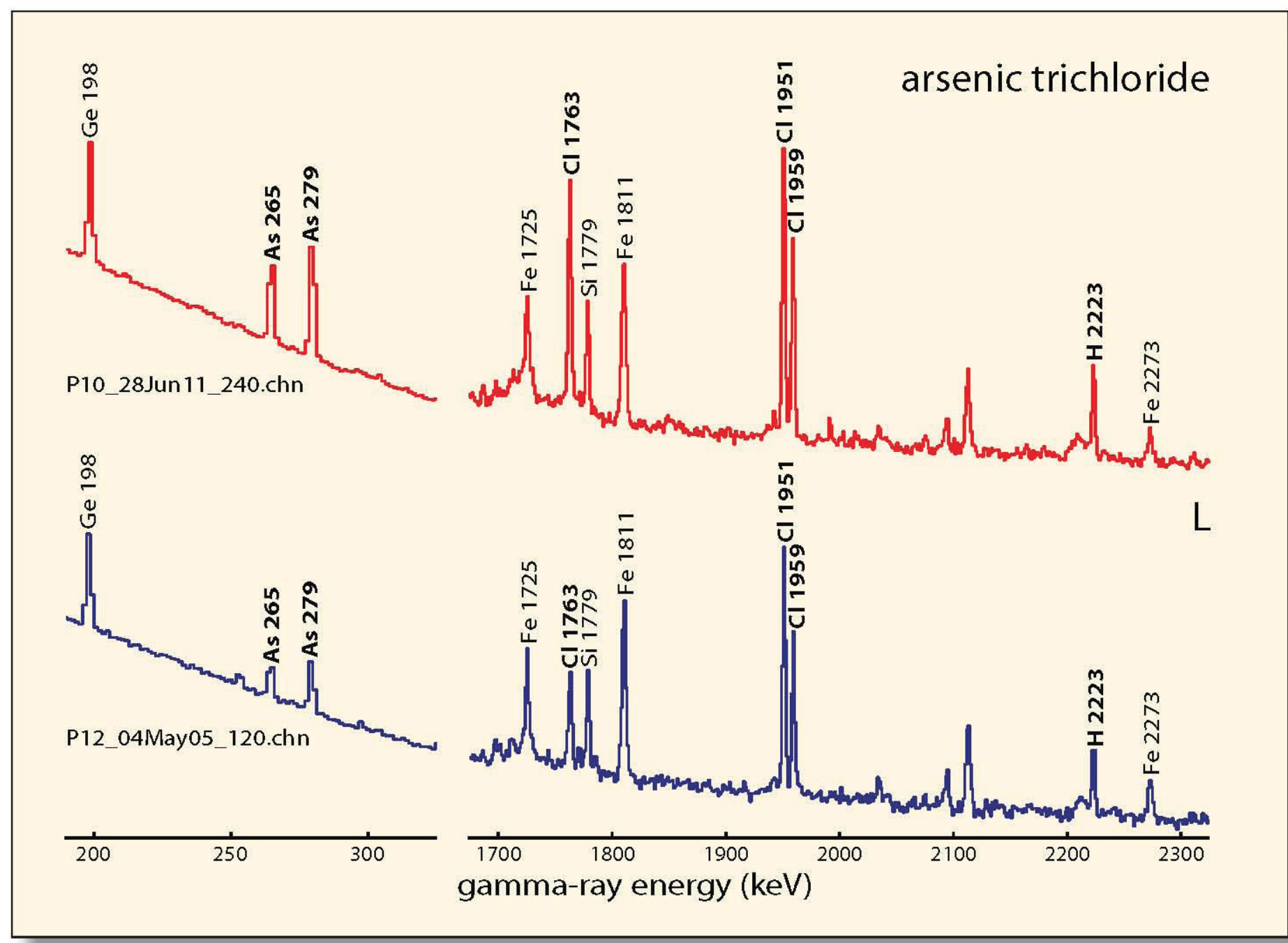

\section{Spectral comparison}

Arsenic trichloride simulant (red curve) and L blister agent (blue) PINS spectra are compared in the figure above. Both spectra were measured from 4.2 -inch mortar projectiles.

\section{Distinguishing features}

- Strong-to-very strong arsenic peaks are observed in both the arsenic trichloride and L spectra at 265 and $279 \mathrm{keV}$.

- Strong chlorine peaks are seen in both the arsenic trichloride and L spectra at 1763, 1951, and $1959 \mathrm{keV}$.
- The arsenic-to-chlorine (279/1959) peak ratio is about 6.5 for $\mathrm{L}$, and about 9 for $\mathrm{AsCl}_{3}$.

- The chlorine inelastic-to-capture (1763/1959) peak ratio is less than 1 for $L$, but greater than 1 for arsenic trichloride.
Chemical name: arsenic trichloride

Chemical formula: $\mathrm{AsCl}_{3}$

Key elements: arsenic $41.3 \mathrm{wt}-\%$, chlorine $58.7 \mathrm{wt}-\%$

Key element ratios: (As 279)/(Cl 1959) $=9.3 \pm 1.2$

$(\mathrm{Cl} 1763) /(\mathrm{Cl} 1959)=1.2 \pm 0.2$
NATO Symbol: $n$ /a

Chemical type: agent precursor

CWC schedule: $2 \mathrm{~B}$

AKA: butter of arsenic

fuming liquid arsenic 


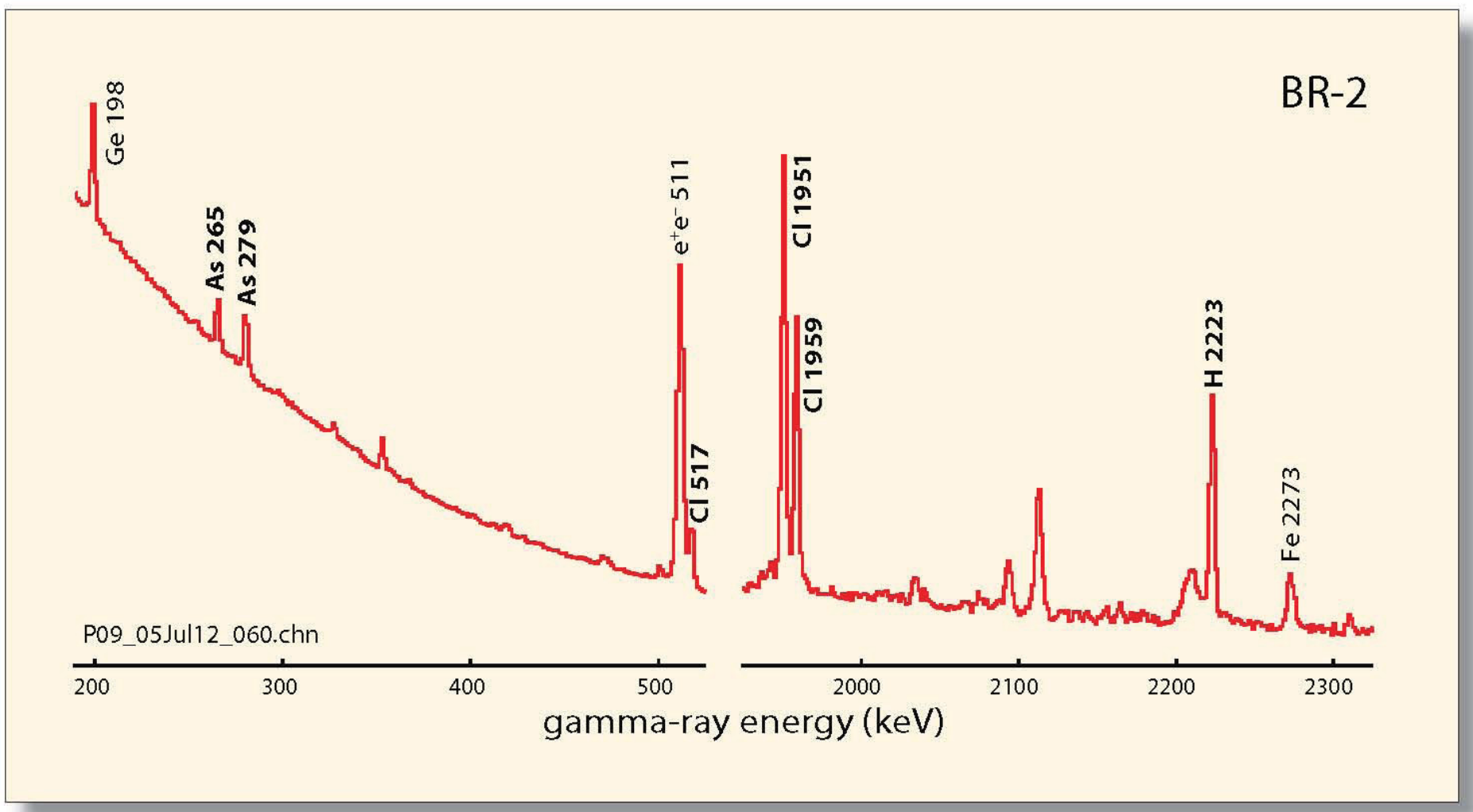

\section{Key spectral features}

- Strong or very strong As peaks at 265 and $279 \mathrm{keV}$

- Moderate or strong chlorine peaks at 517, 1763, 1951, and $1959 \mathrm{keV}$

- Moderate or strong hydrogen peak at $2223 \mathrm{keV}$

\section{Spectrum ID Tips}

- Arsenic peaks are seen at 265 and $279 \mathrm{keV}$, above a slanting Compton continuum.

- The 279-keV arsenic peak is approximately equal to the 517-keV chlorine peak, and it is 3.5 times as strong as the 1959-keV chlorine peak.

- The 1959-keV chlorine peak is about twice as strong as the 2223-keV hydrogen peak.

\section{Caveats}

- The $(\mathrm{Cl} 1959) /(\mathrm{H} 2223)$ ratio may vary, depending on background subtraction effects.
Chemical names: phenyldichloroarsine (PD), 27\% diphenylchloroarsine (DA), $59 \%$

Chemical formula: $\mathrm{PD}_{6} \mathrm{C}_{6} \mathrm{H}_{5} \mathrm{AsCl}_{2} \mathrm{DA}_{4} \mathrm{C}_{12} \mathrm{H}_{10} \mathrm{AsCl}$

Key elements: As 33.2 wt- $\%$, Cl 17.1 wt- $\%, \mathrm{H} 3.3$ wt- $\%$

Key element ratio: (As 279) $/(\mathrm{Cl} 1959)=3.5 \pm 0.5$
NATO Symbol: DA-PD

Chemical type: blister agent, vomiting agent

CWC schedule: 1

AKA: DA-PD, BR-2 


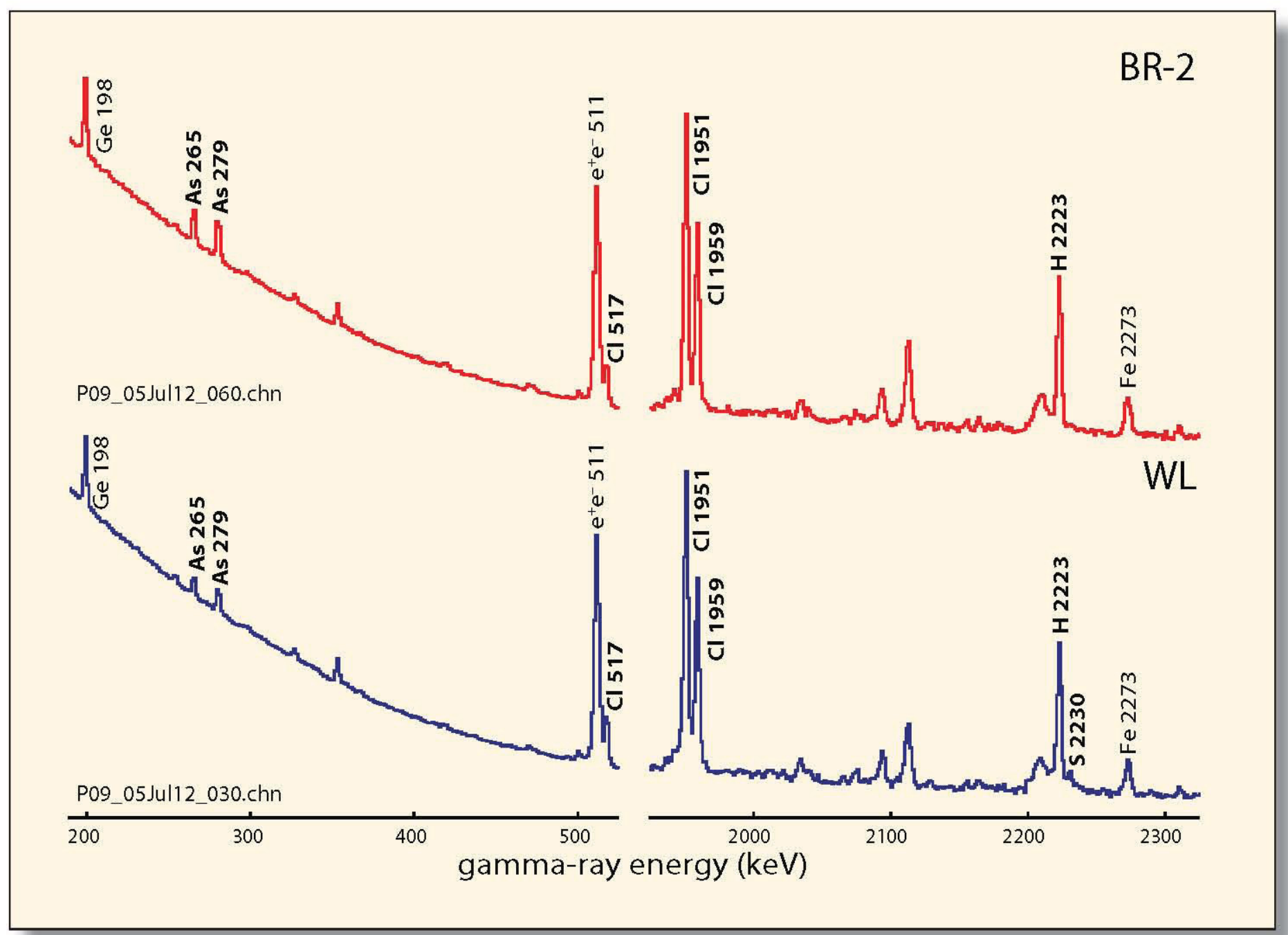

\section{Spectral comparison}

The arsenic and chlorine-hydrogen spectral regions of agent blue ring- 2 (red curve) and WL blister agent (blue) are compared in the figure above. Both PINS spectra were measured from simulant chemicals produced at Idaho National Laboratory.

\section{Distinguishing features}

- A weak sulfur peak is seen in WL at $2230 \mathrm{keV}$, but not in BR2.

- For WL, the intensity of the $279-\mathrm{keV}$ As peak is roughly half that of the 517-keV chlorine peak, but for BR2, the arsenic peak is approximately equal in intensity to the chlorine peak.
Chemical names: phenyldichloroarsine (PD), 27\% diphenylchloroarsine (DA), 59\%

Chemical formula: $\mathrm{PD}, \mathrm{C}_{6} \mathrm{H}_{5} \mathrm{AsCl}_{2} \mathrm{DA}_{4} \mathrm{C}_{12} \mathrm{H}_{10} \mathrm{AsCl}$

Key elements: As 33.2 wt- $\%$, Cl 17.1 wt $\%, \mathrm{H} 3.3$ wt- $\%$

Key element ratio: (As 279) $/(\mathrm{Cl} 1959)=3.5 \pm 0.5$
NATO Symbol: DA-PD

Chemical type: blister agent, vomiting agent

CWC schedule: 1

AKA: DA-PD, BR-2 


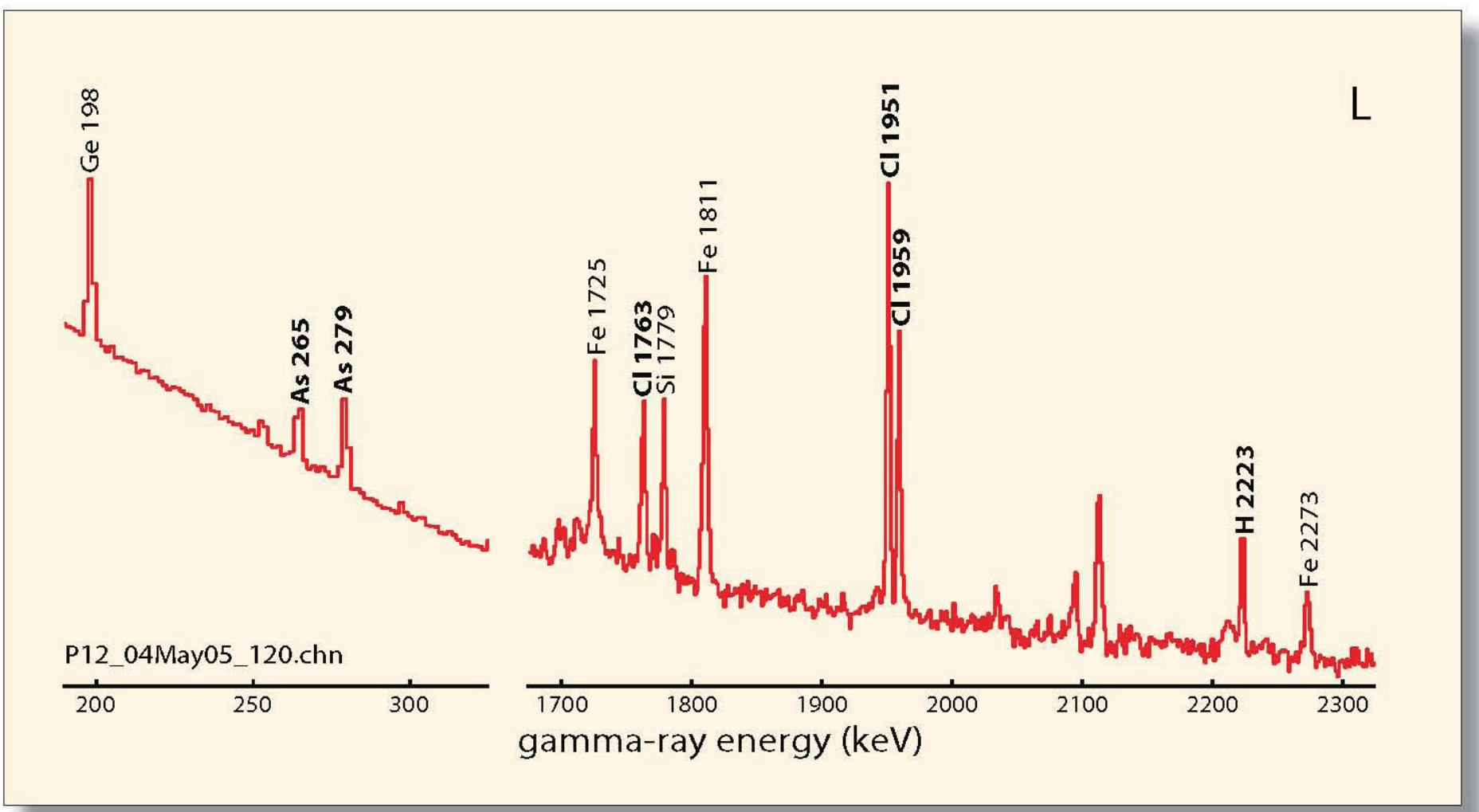

\section{Key spectral features}

- Strong or very strong As peaks at 265 and $279 \mathrm{keV}$

- Moderate or strong chlorine peaks at 1763,1951 , and $1959 \mathrm{keV}$

\section{Spectrum ID Tips}

- Arsenic peaks are seen at 265 and $279 \mathrm{keV}$, above a slanting Compton continuum.

- Lewisite contains $1 \%$ hydrogen, and its chlorine inelasticto-capture ratio is about 0.9 .

- The lewisite (As 279)/(Cl 1959) ratio is approximately 6.5 .

\section{Caveats}

- The $L$ hydrogen peak intensity is often zero, due to background over-subtraction.

- This spectrum was measured from a 4.2 inch mortar projectile.

- PINS spectra have been measured from less than a dozen L-filled containers and munitions.
Chemical names: dichloro(2-chlorovinyl)arsine Chemical formula: $\mathrm{C}_{2} \mathrm{H}_{2} \mathrm{AsCl}_{3}$

Key elements: As 36.1 wt- $\%, \mathrm{Cl} 51.3 w \mathrm{wt} \%, \mathrm{H} 1.0 \mathrm{wt}-\%$

Key element ratios: $($ As 279) $/(\mathrm{Cl} 1959)=6.5 \pm 1.5$

$(C l 1973) /(C l 1959)=0.9 \pm 0.3$
NATO Symbol: L

Chemical type: blister agent

CWC schedule: 1

AKA: lewisite 


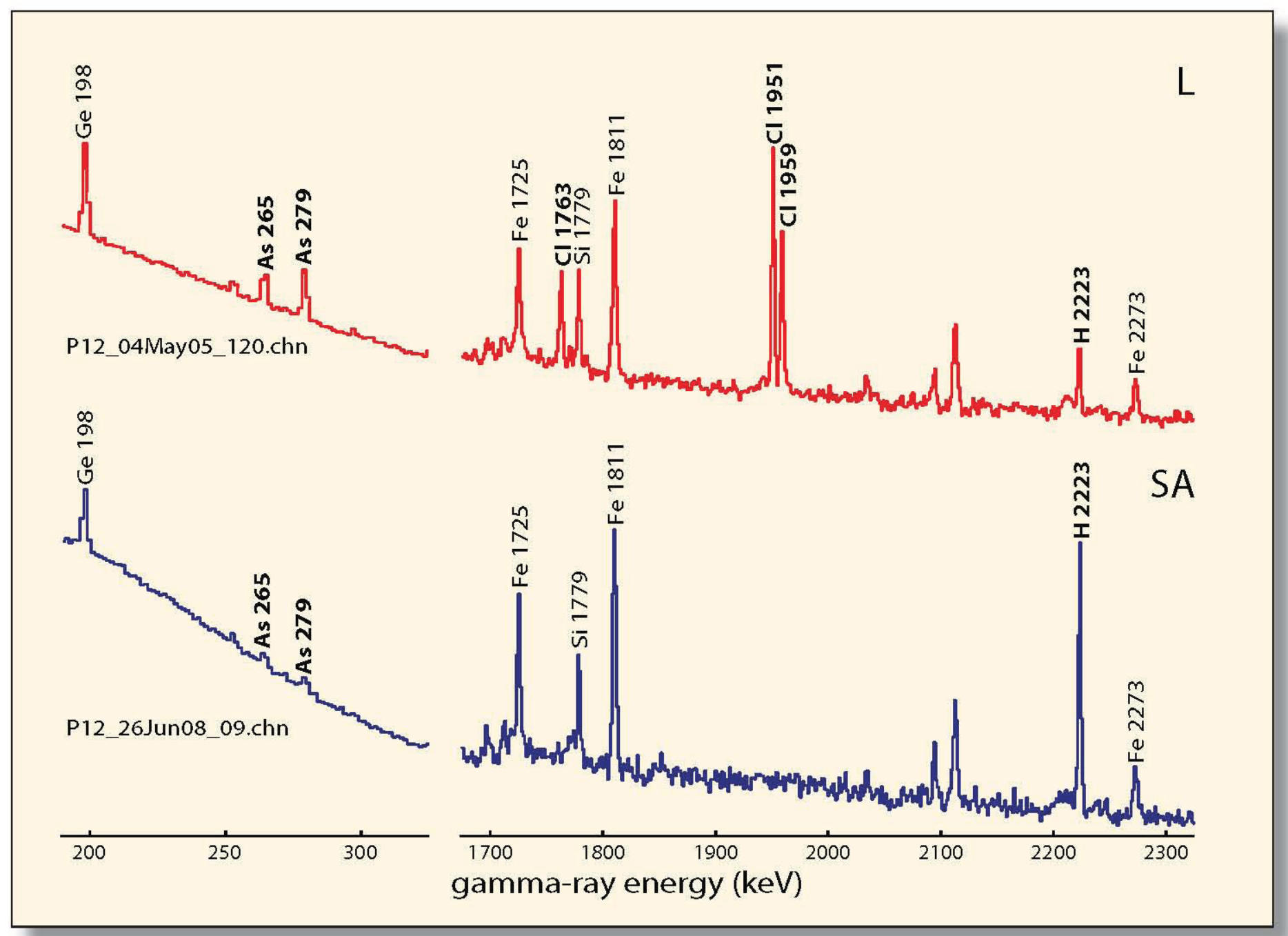

\section{Spectral comparison}

The arsenic and chlorine-hydrogen spectral regions of SA blood agent (blue curve) and L blister agent (red) are compared in the figure above. The SA PINS spectrum was measured from a 75- $\mathrm{mm}$ artillery projectile, and the $\mathrm{L}$ spectrum from a 4.2-inch mortar projectile.

\section{Distinguishing features}

- Moderate-to-strong arsenic peaks are observed in both the SA and L spectra at 265 and $279 \mathrm{keV}$.

- Strong chlorine peaks are seen in the $L$ spectrum at 1763,1951 , and $1959 \mathrm{keV}$.

- No chlorine peaks are observed in the SA spectrum.
Chemical names: dichloro(2-chlorovinyl)arsine

Chemical formula: $\mathrm{C}_{2} \mathrm{H}_{2} \mathrm{AsCl}_{3}$

Key elements: As 36.1 wt- $\%, \mathrm{Cl} 51.3$ wt- $\%, \mathrm{H} 1.0$ wt- $\%$

Key element ratios: (As 279)/(Cl 1959) $=6.5 \pm 1.5$

$(\mathrm{Cl} 1973) /(\mathrm{Cl} 1959)=0.9 \pm 0.3$
NATO Symbol: L

Chemical type: blister agent

CWC schedule: 1

AKA: lewisite 


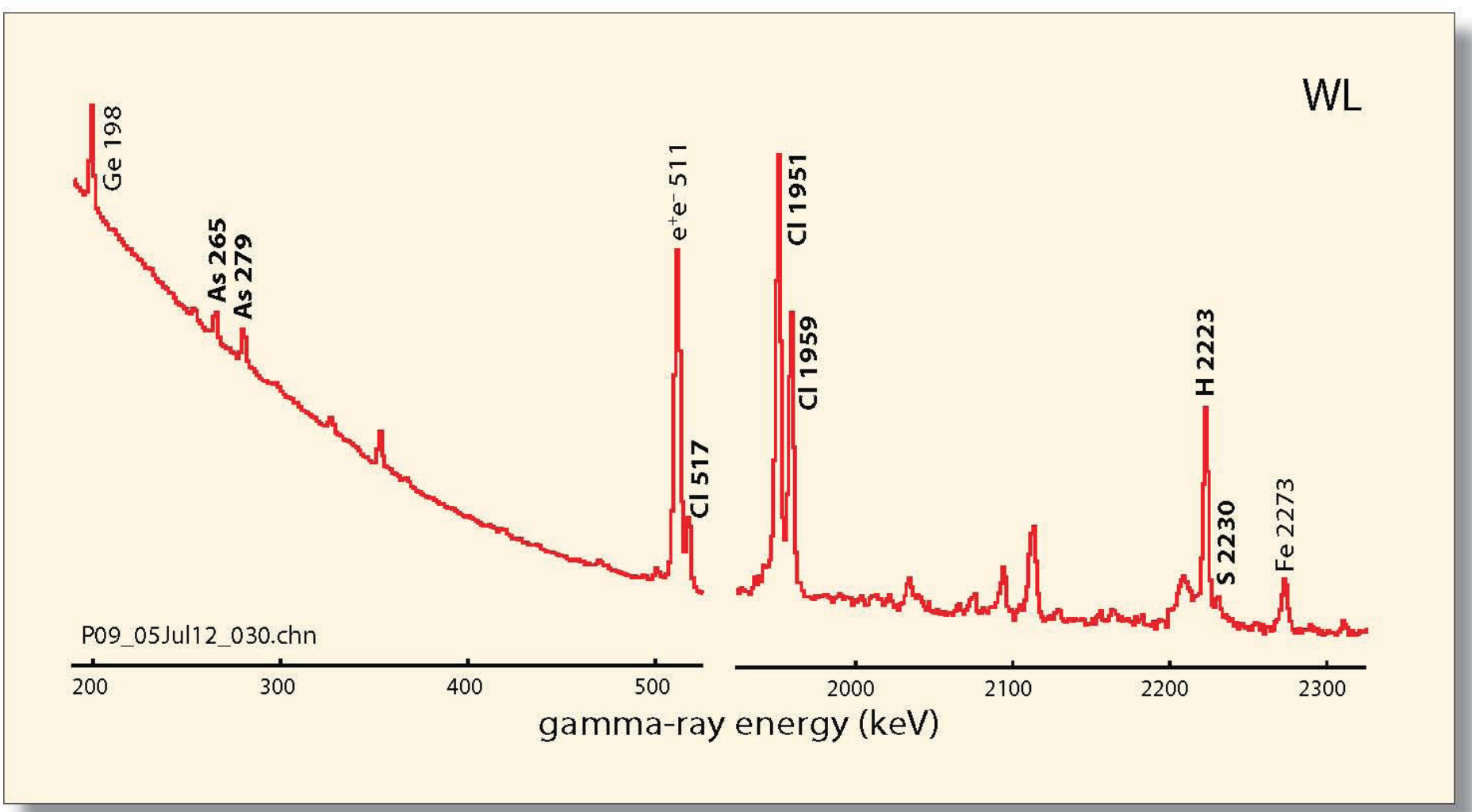

\section{Key spectral features}

- Strong or very strong As peaks at 265 and $279 \mathrm{keV}$

- Moderate or strong chlorine peaks at 1763, 1951, and $1959 \mathrm{keV}$

- Moderate or strong hydrogen peak at $2223 \mathrm{keV}$

- Weak sulfur peak at $2230 \mathrm{keV}$

\section{Spectrum ID Tips}

- Arsenic peaks are seen at 265 and $279 \mathrm{keV}$, above a slanting Compton continuum.

- The 279-keV arsenic peak area is roughly half that of the 517-keV chlorine peak area, and it is about 1.7 times stronger than the 1959-keV chlorine peak.

- A weak or moderate sulfur peak will be observed at $2230 \mathrm{keV}$.

\section{Caveats}

- The (Cl 1959)/(H 2223) peak area ratio may vary, depending on background subtraction effects.
Chemical name: Winterlöst (winter mustard)

Chemical mixture: $\mathrm{H}$, bis (2-chloroethyl) sulfide, $50 \%$

$$
\mathrm{PD} \text {, phenyldichloroarsine, } 50 \%
$$

Key elements: As 20.0 wt- $\%, \mathrm{Cl} 31.7, \mathrm{H} 3.9$ wt- $\%, \mathrm{~S} 10.1$ wt- $\%$

Key element ratio: $($ As 279$) /(\mathrm{Cl} 1959)=1.7 \pm 0.1$
NATO Symbol: $n / a$

Chemical type: blister agent

CWC schedule: 1

AKA: winter mustard, H-PD, WL 


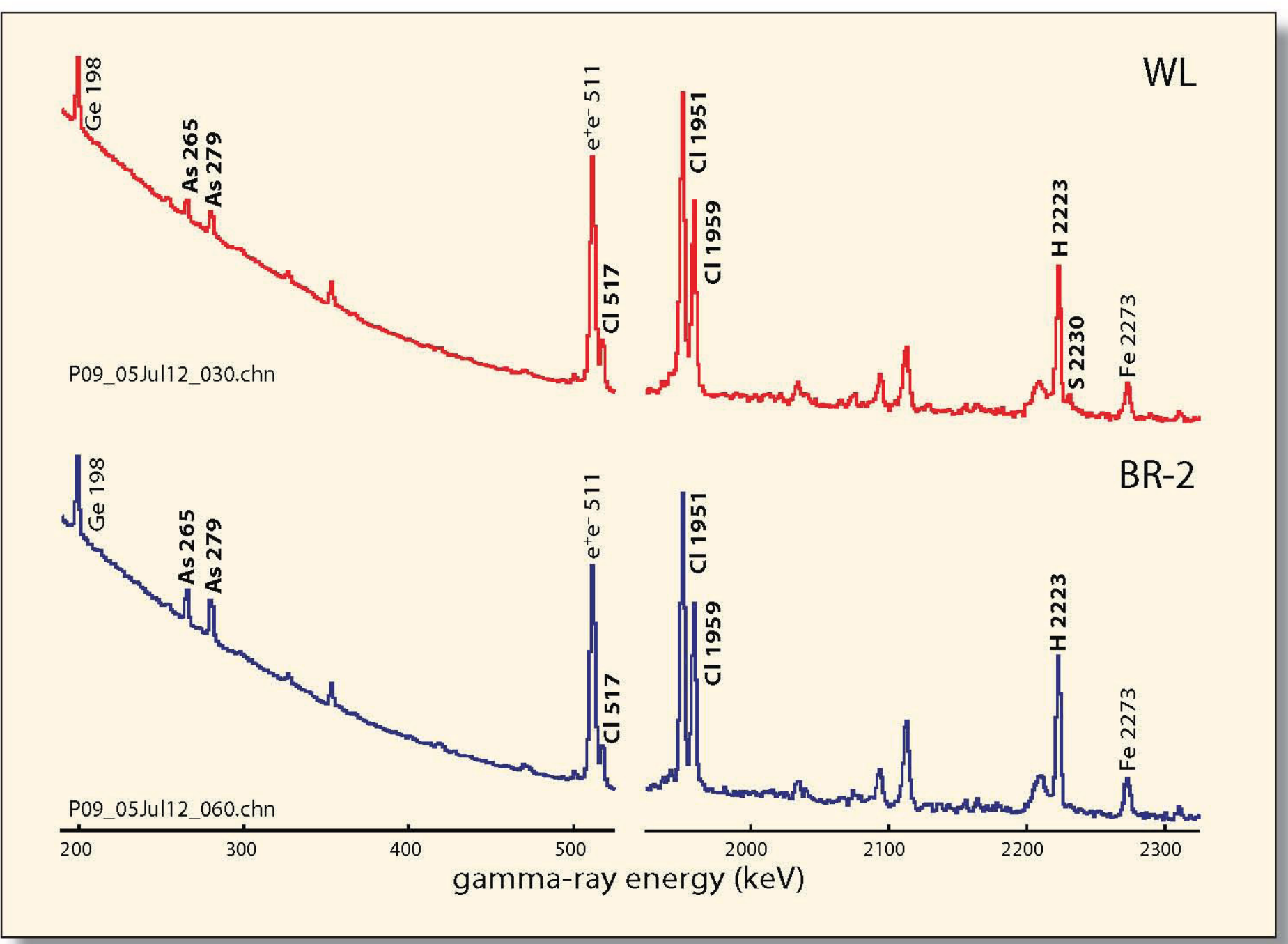

\section{Spectral comparison}

The arsenic and chlorine-hydrogen spectral regions of WL blister agent (red curve) and agent blue ring-2 (blue curve) are compared in the figure above. Both PINS spectra were measured from simulant chemicals produced at Idaho National Laboratory.

\section{Distinguishing features}

- A weak sulfur peak is seen in WL at $2230 \mathrm{keV}$, but not in BR2.

- For $W L$, the intensity of the $279-k e V$ As peak is roughly half that of the 517-keV chlorine peak, but for BR2, the arsenic peak is approximately equal in intensity to the chlorine peak.
Chemical name: Winterlöst (winter mustard)

Chemical mixture: $\mathrm{H}$, bis (2-chloroethyl) sulfide, $50 \%$

$\mathrm{PD}_{\text {, }}$ phenyldichloroarsine, $50 \%$

Key elements: As $20.0 \mathrm{wt}-\%, \mathrm{Cl} 31.7, \mathrm{H} 3.9 \mathrm{wt}-\%, \mathrm{~S} 10.1 \mathrm{wt}-\%$

Key element ratio: $($ As 279) $/(\mathrm{Cl} 1959)=1.7 \pm 0.1$

\section{NATO Symbol: $n / a$}

Chemical type: blister agent

CWC schedule: 1

AKA: winter mustard, H-PD, WL 


\section{Chapter 7 \\ Chlorine-Based Chemicals}

\section{Smoke Chemicals}

The use of obscuring smoke is probably as old as organized warfare. Smoke chemicals are used to adjust artillery fire and to obscure movement on the battlefield. Several smoke chemicals are based on chlorine: FM, FS, HC, and KJ. All are fast and easy to identify with PINS. Typically the chlorine peaks become evident in the spectrum first, but within a minute, the other key element peaks can usually be seen.

Two smoke chemicals are binary chlorine compounds. FM smoke is titanium tetrachloride, and $\mathrm{KJ}$ smoke is tin tetrachloride.[1]

$\mathrm{HC}$ smoke is a mixture of aluminum powder, zinc oxide, and hexachloroethane.

FS, the fourth chlorine-based smoke chemical, is a mixture of sulfur trioxide dissolved in chlorosulfonic acid.[2]

\section{Blister Agents}

Two blister agents are chlorine based: sulfur mustard, agent $\mathrm{H}$,[3] and nitrogen mustard, agent HN.[4] As the names suggest, sulfur mustard contains sulfur, but no nitrogen, and nitrogen mustard contains nitrogen but no sulfur. In this Guide, the word "mustard" without qualification refers to sulfur mustard.

\section{Blood Agent}

Agent CK, a chlorine-base cyanide, poisons by interfering with oxygen transfer in the blood stream. [5]

\section{Riot Agents}

The riot agents $\mathrm{CNB}$ and $\mathrm{CNS}$ are based on the tearproducing chemical chloroacetophenone, [6] or more commonly, Mace. The NATO designation of Mace is " $\mathrm{CN}$ ". $\mathrm{CNB}$ is agent $\mathrm{CN}$ dissolved in a mixture of carbon tet and benzene. $\mathrm{CNS}$ is agent $\mathrm{CN}$ and chloropicrin (agent PS) dissolved in chloroform. [7]

\section{Choking Agent}

Phosgene, agent CG, when inhaled reacts with moisture in the lungs to produce hydrochloric acid. The acid in turn destroys lung tissue and produces pulmonary edema.[8]

\section{Spectrum Identification}

Figure 7-1 illustrates the spectrum identification logic for smoke chemicals and mustard blister agent. The smoke chemicals $\mathrm{KJ}, \mathrm{FM}$, and $\mathrm{HC}$ are uniquely identified by the observation of chlorine gamma-ray peaks plus the gammaray peaks of tin, titanium, or zinc respectively.

The elements chlorine and sulfur are common to both FS smoke and mustard agent. However the two chemicals can be easily distinguished by the $\mathrm{Cl} / \mathrm{S}$ ratio, that is, the ratio of the chlorine $1959-\mathrm{keV}$ peak area to the sulfur $2230-\mathrm{keV}$ peak area. If the two peaks are approximately equal in area $(\mathrm{Cl} / \mathrm{S}=1)$, the fill chemical is FS. If the chlorine peak is about twelve times bigger than the sulfur peak $(\mathrm{Cl} / \mathrm{S}=12)$, the fill chemical is mustard agent.

The identification logic for the remaining chlorine based chemicals identified by PINS is shown in Figure 7-2. If nitrogen is detected in addition to chlorine, there are two

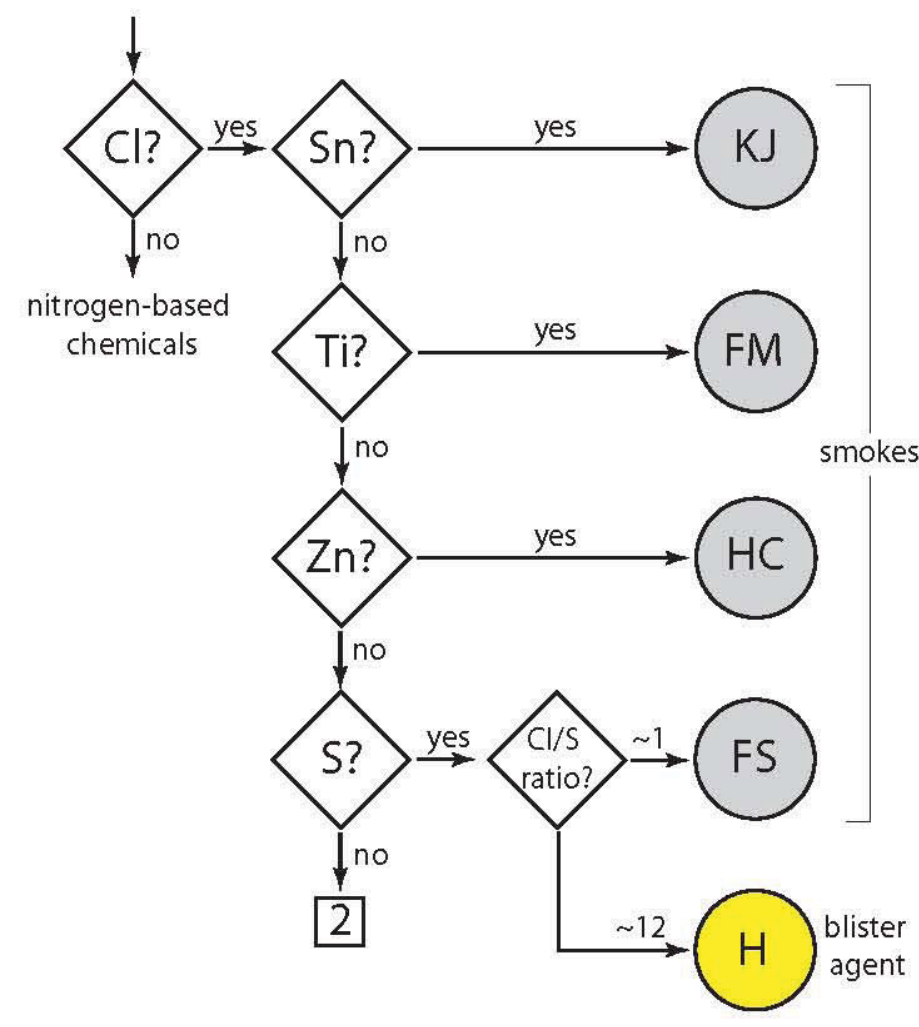

Figure 7-1: PINS identification logic for smoke chemicals and sulfur mustard agent. 
possible fills, $\mathrm{HN}$ and $\mathrm{CK}$. CK contains no hydrogen, while HN produces a strong hydrogen peak.

The remaining chlorine-based chemicals are identified on the basis of the chlorine inelastic-to-capture $(\mathrm{Cl} \mathrm{i} / \mathrm{c})$ ratio, that is the ratio of the $\mathrm{Cl} 1763-\mathrm{keV}$ peak area to that of the $\mathrm{Cl} 1959-\mathrm{keV}$ peak area. In chemicals that contain no hydrogen, inelastic neutron scattering is enhanced, while neutron capture is reduced, producing a large inelasticto-capture ratio in chlorinated chemicals. The opposite is true in chemicals that contain a lot of hydrogen, and hence the inelastic ratio is a sensitive measure of the presence of hydrogen in chlorinated chemicals. CG typically has an inelastic ratio over $1.0, \mathrm{CNS}$ a ratio of about 0.4 , and the $\mathrm{CNB}$ ratio is approximately 0.1 .

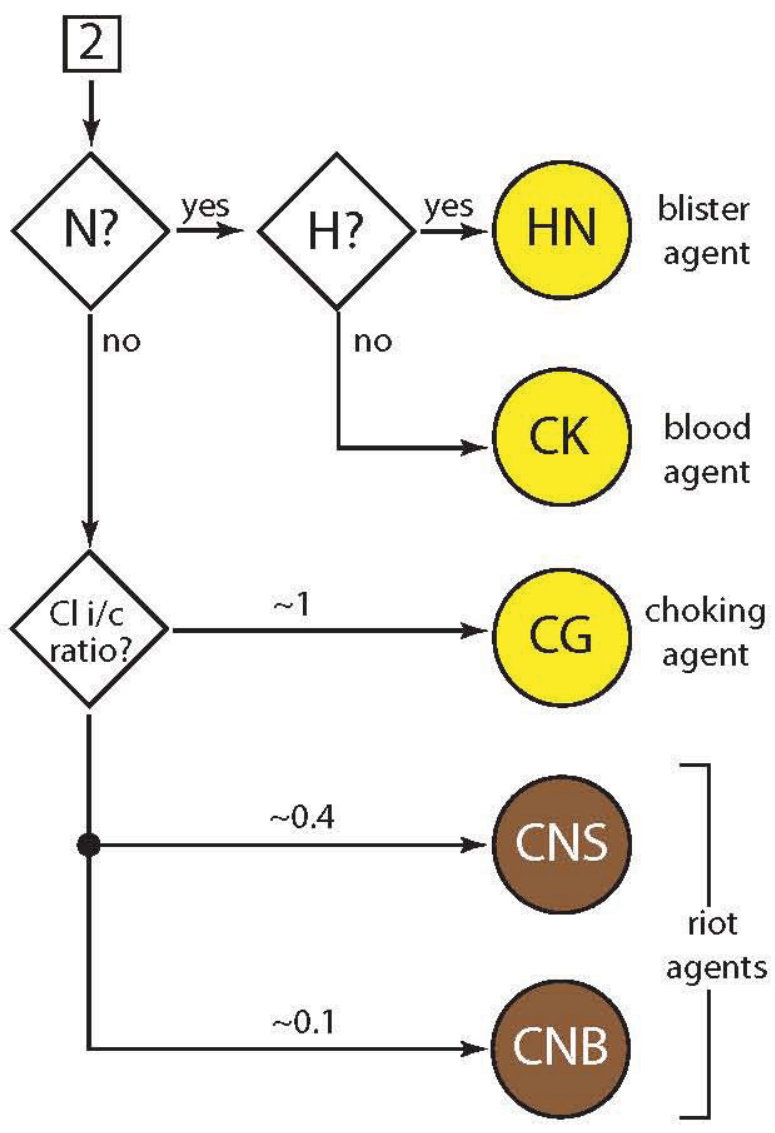

Figure 7-2: PINS identification logic for the chlorine-based chemicals $C G, C K, C N B, C N S$, and $H N$. The abbreviation " $\mathrm{Cli} / \mathrm{Cratio}$ " stands for "chlorine inelastic-to-capture ratio".

\section{References}

1. Military Chemistry and Chemical Compounds, U.S. Army Field Manual 3-9, October 1975, pages 3-34 and 3-36. A.M. Prentiss, Chemicals in War (New York: McGraw-Hill, 1937) pages 238-9.

2. FM 3-9, 1975, page 3-35.

3. Potential Military Chemical/Biological Agents and Compounds, U.S. Army Field Manual 3-11.9 (2005) pages II-37 to II-40 and II-48 to II-50. James A.F. Compton, Military Chemical and Biological Agents (Caldwell, NJ: The Telford Press, 1987) pages 9 to 16.

4. FM 3-11.9, pages II-40 to II-48. Compton, pages 45 to 62 .

5. FM 3-11.9 pages II-33 to II-35. Compton, pages 95 to 99 .

6. Prentiss, pages 142 to 144 .

7. FM 3-11.9, pages III-14 to III-16. Compton, pages 215-236.

8. FM 3-11.9, pages II-9 to II-13. Compton, pages 118-123. 


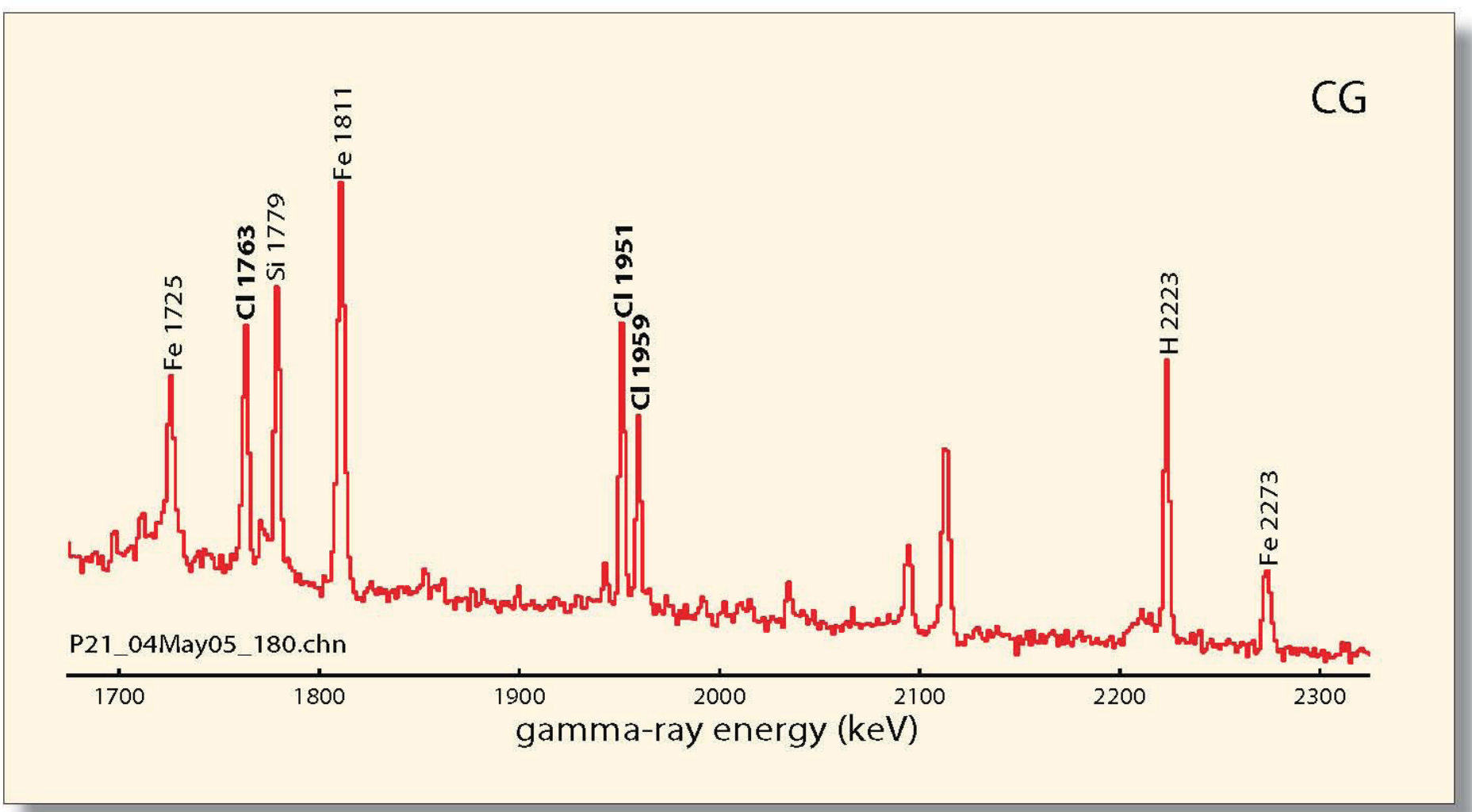

\section{Key spectral features}

- Moderate or strong chlorine peaks at 1763, 1951, and $1959 \mathrm{keV}$

- Only background-level hydrogen peak

\section{Spectrum ID Tips}

- Chlorine peaks will be evident in the PINS spectrum within 100 seconds or less.

- The 1763-keV Cl inelastic peak is equal to or stronger than the 1959-keV Cl capture peak.

- The 2223-keV hydrogen peak is entirely due to background.

\section{Caveats}

- The indicated hydrogen signal may be non-zero due to background under-subtraction.

- Be sure to continue the assay for at least 1,000 seconds, to rule out other possible chlorine-based chemicals.

- This spectrum was measured from a 4.2-inch mortar projectile containing diphosgene. The diphosgene (DP) elemental concentrations are identical to those of CG.

- PINS spectra have been measured from less than a dozen CG-filled containers and munitions.
Chemical name: phosgene

Chemical formula: $\mathrm{COCl}_{2}$

Key elements: chlorine $71.7 \mathrm{wt}-\%$

Key element ratios: $(\mathrm{Cl} 1763) /(\mathrm{Cl} 1959)=0.93 \pm 0.04$
NATO Symbol: CG

Chemical type: choking agent

CWC schedule: 3

AKA: carbonyl chloride 


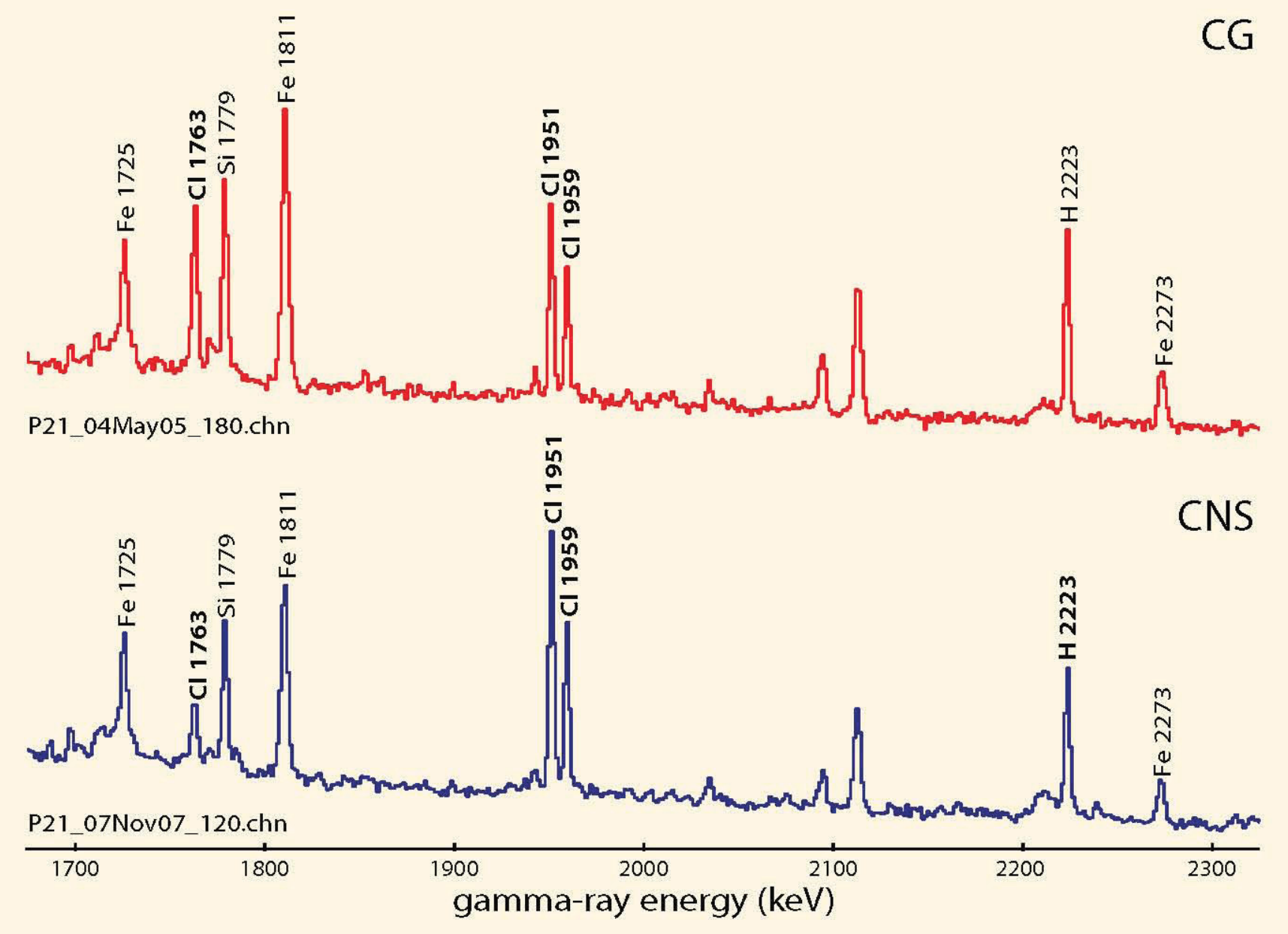

\section{Spectral comparison}

The figure above compares diphosgene, itself a choking

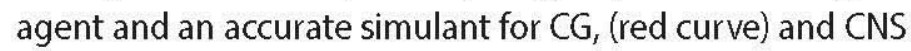
riot agent (blue). Both spectra were measured from 4.2inch mortar projectiles.

\section{Distinguishing features}

- Strong chlorine peaks are seen in both the CG and CNS spectra at 1763, 1951, and $1959 \mathrm{keV}$.

- The chlorine inelastic-to-capture (Cl 1763/Cl 1959) peak ratio is about 1.0 for $\mathrm{CG}$, but about 0.4 for CNS.
Chemical name: phosgene

Chemical formula: $\mathrm{COCl}_{2}$

Key elements: chlorine 71.7 wt- $\%$

Key element ratios: $(\mathrm{Cl} 1763) /(\mathrm{Cl} 1959)=0.93 \pm 0.04$
NATO Symbol: CG

Chemical type: choking agent

CWC schedule: 3

AKA: carbonyl chloride 


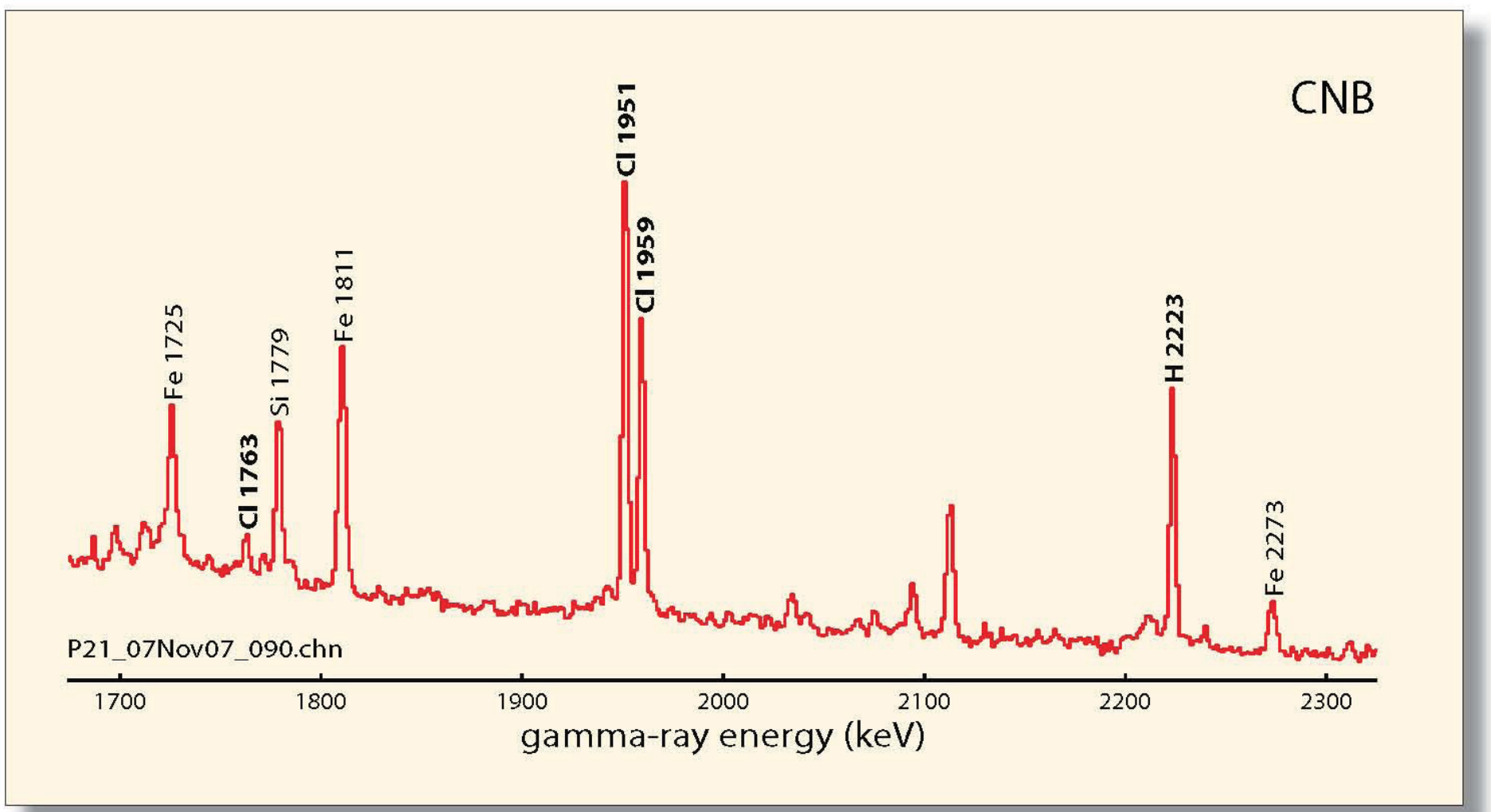

\section{Key spectral features}

- Moderate or strong chlorine peaks at 1763, 1951, and $1959 \mathrm{keV}$

- Moderate to strong hydrogen peak at $2223 \mathrm{keV}$

\section{Spectrum ID Tips}

- Chlorine peaks will be evident in the PINS spectrum within 100 seconds or less.

- The 1763-keV Cl inelastic peak area is almost a factor of ten smaller than the 1959-keV Cl capture peak.

\section{Caveats}

- Background over- or under-subtraction may render the hydrogen peak area inaccurate.

- This agent is best identified by the absence of other key elements, and by its characteristic chlorine inelastic-tocapture ratio.

- This spectrum was measured from a 4.2-inch mortar projectile.

- PINS spectra have been measured from less than a dozen CNB-filled munitions.
Chemical mixture: chloroacetophenone, carbon tetrachloride, and benzene

Chemical formulae: $\mathrm{C}_{8} \mathrm{H}_{7} \mathrm{ClO} 10 \mathrm{wt} . \%, \mathrm{CCl}_{4} 45$ wt. $-\%, \mathrm{C}_{6} \mathrm{H}_{6} 45$ wt. $\%$ Key elements: chlorine 43.8 wt- $\%$, hydrogen $3.9 w t-\%$
Key element ratios: $(\mathrm{Cl} 1763) /(\mathrm{Cl} 1959)=0.13 \pm 0.02$

NATO Symbol: CNB

Chemical type: riot agent

CWC schedule: $n / a$ 

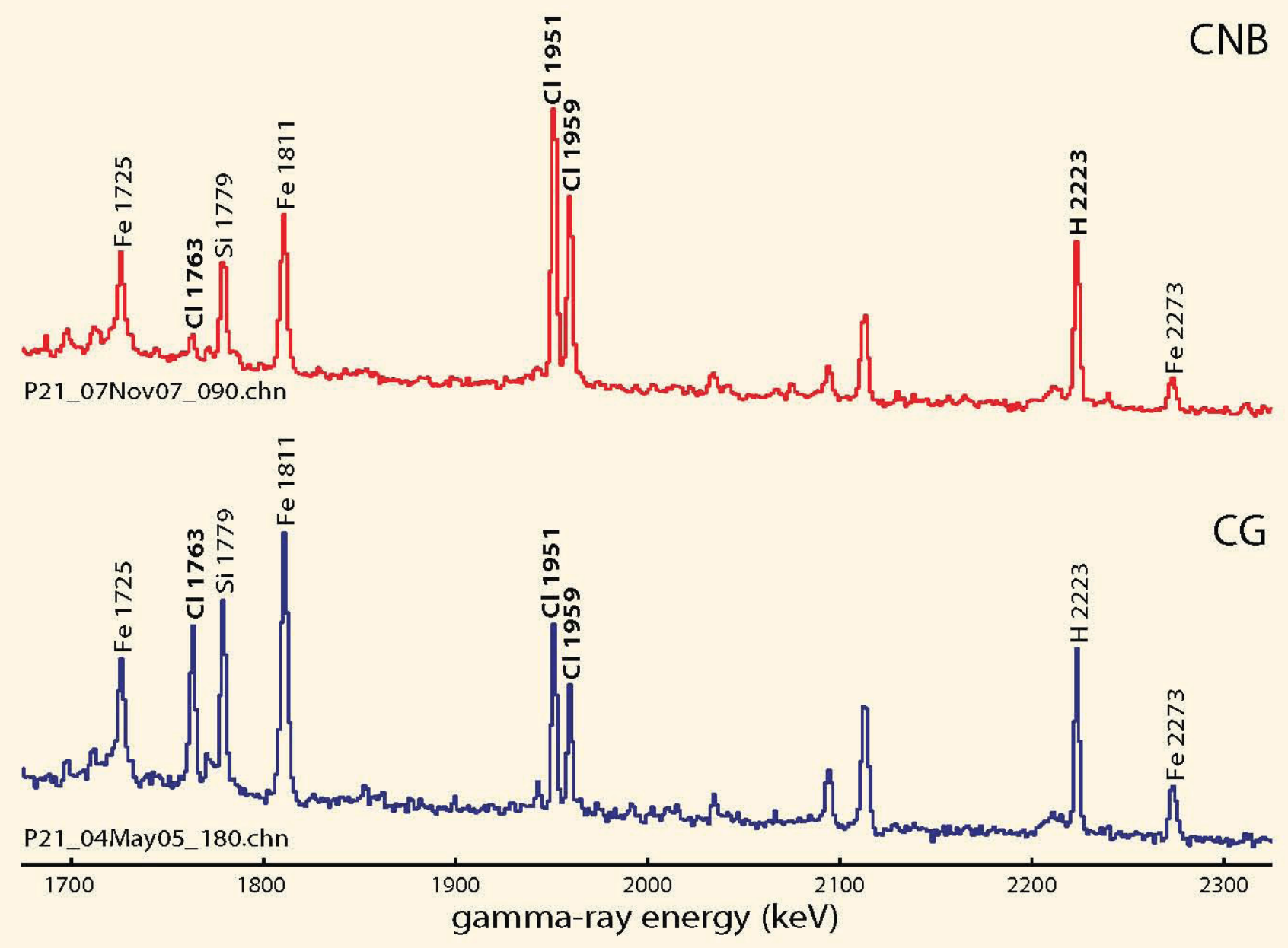

\section{Spectral comparison}

The figure above compares agents CNB (red curve) and diphosgene (blue). Diphosgene, itself a choking agent, is an accurate simulant for CG. Both spectra were measured from 4.2-inch mortar projectiles.

\section{Distinguishing features}

- The 2223-keV hydrogen peak is larger for CNB than for CG; the CG hydrogen peak is entirely due to background.

- The chlorine inelastic-to-capture (Cl 1763/Cl 1959) peak ratio is greater than 1.0 for $C G$, but the ratio is about 0.15 for CNB.
Chemical mixture: chloroacetophenone, carbon tetrachloride, and benzene

Chemical formulae: $\mathrm{C}_{8} \mathrm{H}_{7} \mathrm{ClO} 10$ wt.- $\%, \mathrm{CCl}_{4} 45$ wt.- $\%, \mathrm{C}_{6} \mathrm{H}_{6} 45$ wt.- $\%$ Key elements: chlorine 43.8 wt- $\%$, hydrogen 3.9 wt- $\%$
Key element ratios: $(\mathrm{Cl} 1763) /(\mathrm{Cl} 1959)=0.13 \pm 0.02$

NATO Symbol: CNB

Chemical type: riot agent

CWC schedule: $n / a$ 


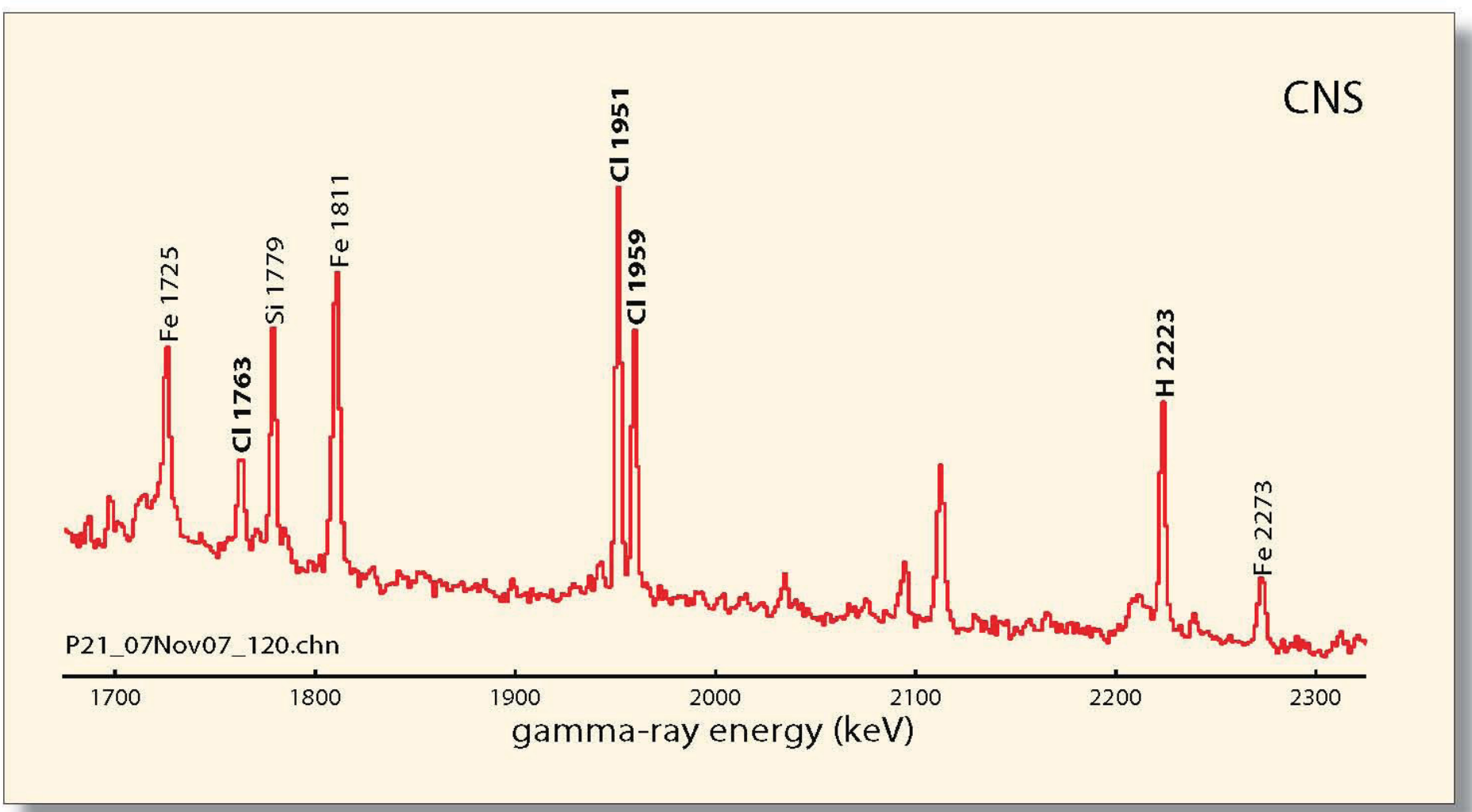

\section{Key spectral features}

- Moderate or strong chlorine peaks at 1763, 1951, and $1959 \mathrm{keV}$

- Moderate hydrogen peak at $2223 \mathrm{keV}$

\section{Spectrum ID Tips}

- Chlorine peaks will be evident in the PINS spectrum within 100 seconds or less.

- The 1763-keV Cl inelastic peak is smaller than the 1959keV Cl capture peak by a factor of two.

\section{Caveats}

- Background over- or under-subtraction may render the hydrogen peak area inaccurate.

- This agent is best identified by the absence of other key elements, and by its characteristic chlorine inelastic-tocapture ratio.

- This spectrum was measured from a 4.2-inch mortar projectile

- PINS spectra have been measured from less than a dozen CNS-filled munitions.
Chemical mixture: chloroacetophenone, chloropicrin, and chloroform

Chemical formulae: $\mathrm{C}_{8} \mathrm{H}_{7} \mathrm{ClO} 24$ wt.- $\%, \mathrm{CNO}_{2} \mathrm{Cl}_{3} 38$ wt.- $\%$, $\mathrm{CHCl}_{3} 38$ wt. $\%$

Key elements: chlorine 64.5 wt- $\%$, hydrogen 1.4 wt- $\%$
Key element ratios: $(\mathrm{Cl} 1763) /(\mathrm{Cl} 1959)=0.39 \pm 0.06$

NATO Symbol: CNS

Chemical type: riot agent

CWC schedule: 3 

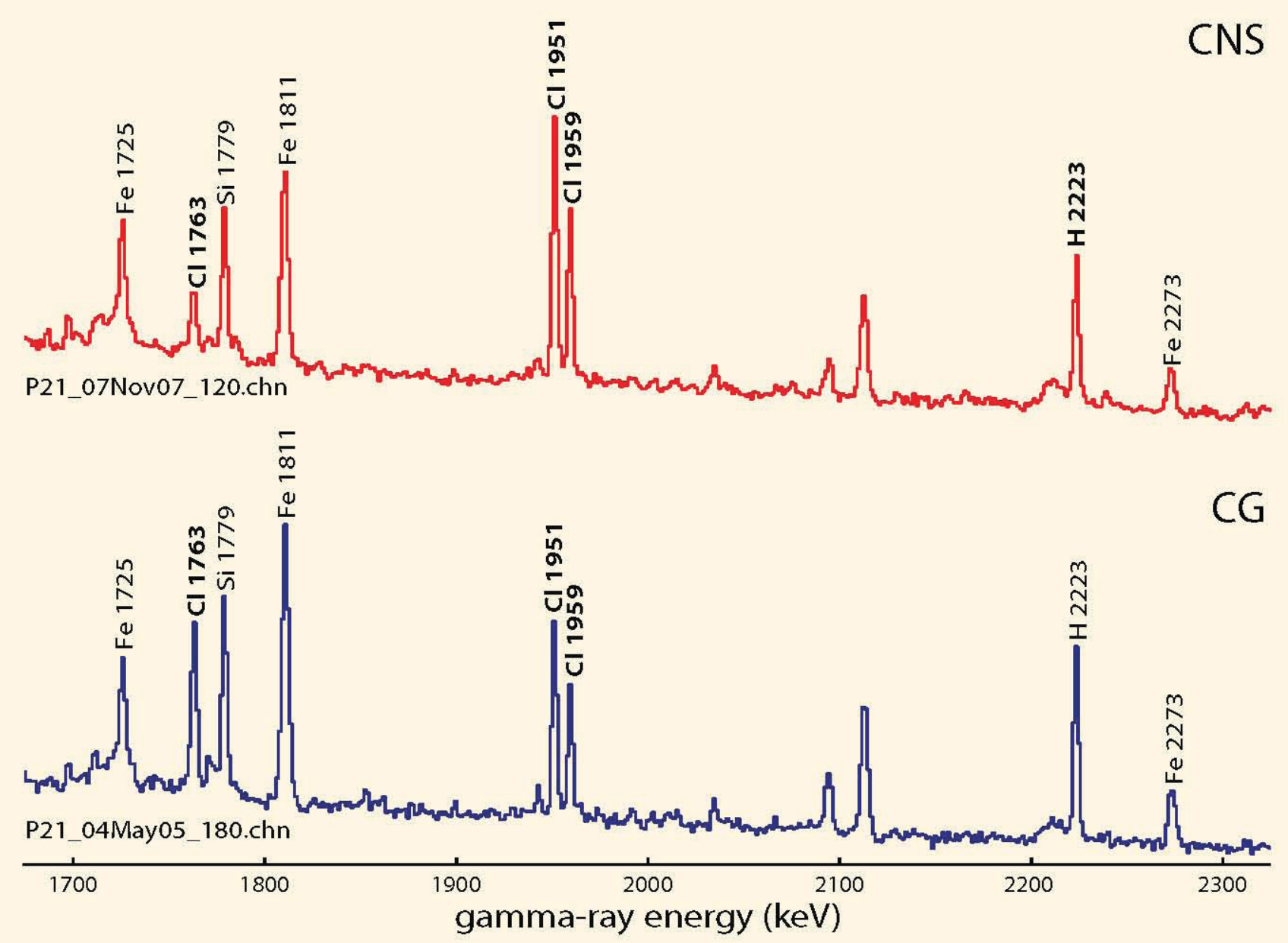

\section{Spectral comparison}

The figure above compares agents CNS (red curve) and diphosgene (blue). Diphosgene, itself a choking agent, is an accurate simulant for CG. Both spectra were measured from 4.2-inch mortar projectiles.

\section{Distinguishing features}

- The 2223-keV hydrogen peak is larger for CNS than for CG; the CG hydrogen peak is entirely due to background.

- The chlorine inelastic-to-capture (Cl 1763/Cl 1959) peak ratio is greater than 1.0 for $C G$, but the ratio is about 0.4 for CNS.
Chemical mixture: chloroacetophenone, chloropicrin, and chloroform

Chemical formulae: $\mathrm{C}_{8} \mathrm{H}_{7} \mathrm{ClO} 24$ wt.- $\%, \mathrm{CNO}_{2} \mathrm{Cl}_{3} 38 \mathrm{wt} .-\%$, $\mathrm{CHCl}_{3} 38$ wt. $\%$

Key elements: chlorine $64.5 \mathrm{wt}-\%$, hydrogen $1.4 \mathrm{wt}-\%$
Key element ratios: $(\mathrm{Cl} 1763) /(\mathrm{Cl} 1959)=0.39 \pm 0.06$

NATO Symbol: CNS

Chemical type: riot agent

CWC schedule: 3 


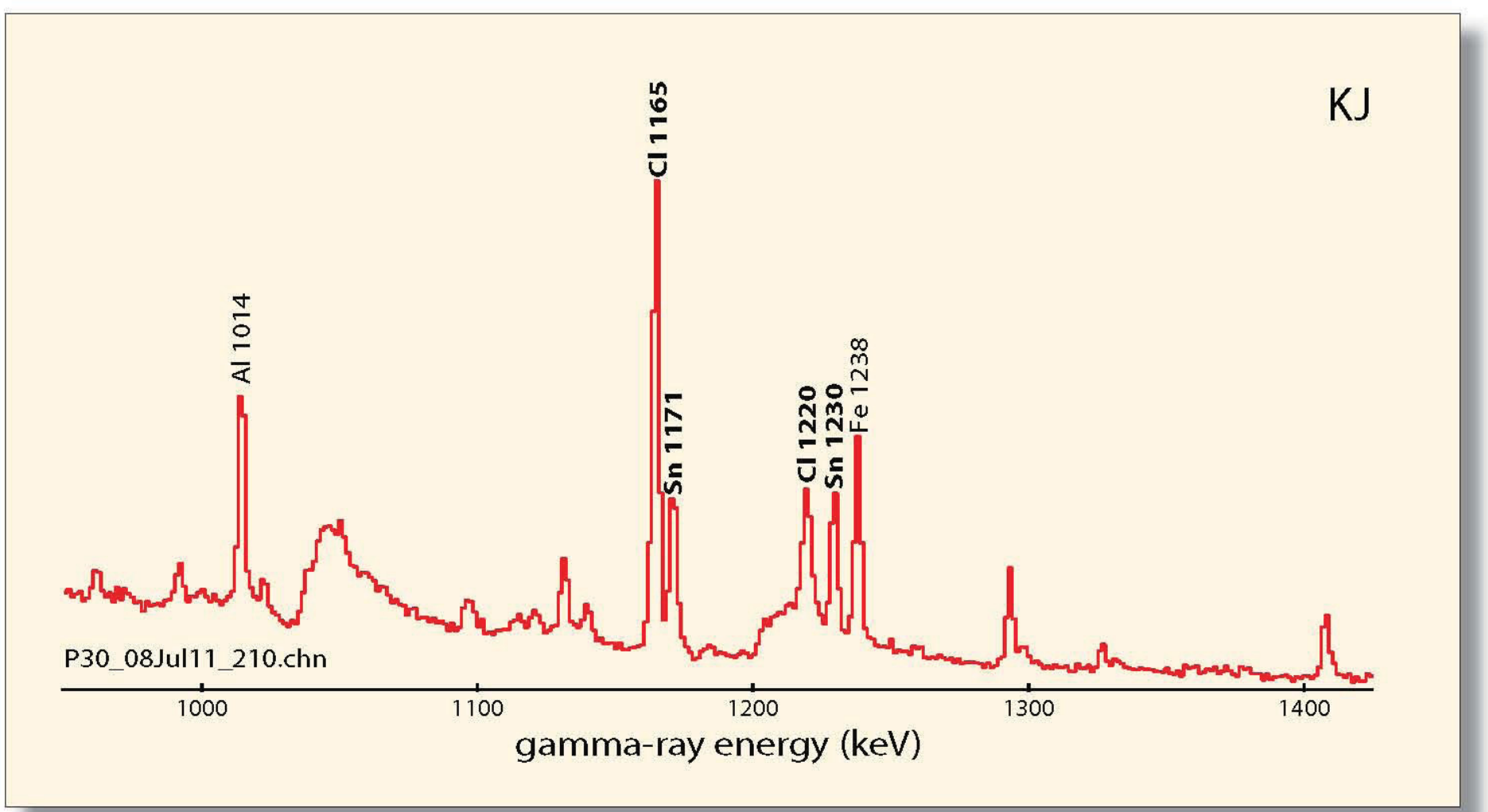

\section{Key spectral features}

- Moderate or strong chlorine peaks at 1164, 1763, 1951, and $1959 \mathrm{keV}$

- Moderate or strong tin peaks at 1171, 1230 and $1294 \mathrm{keV}$

\section{Spectrum ID Tips}

- Chlorine peaks will be evident in the PINS spectrum within 100 seconds or less, and so will the tin peaks.

- The 2223-keV hydrogen peak is entirely due to background.

\section{Caveats}

- The hydrogen peak area can be non-zero if the background is under-subtracted.

- This spectrum was measured from a 4.2 inch mortar projectile replica containing simulated $\mathrm{KJ}$ smoke.

- No PINS spectra have been measured from containers or munitions filled with pure $\mathrm{KJ}$ smoke.

- A handful of munitions containing a mixture of $\mathrm{KJ}$ and a chemical warfare agent have been assayed with PINS.
Chemical name: stannic chloride

Chemical formula: $\mathrm{SnCl}_{4}$

Key elements: chlorine 54.4 wt- $\%$, tin 45.6 wt- $\%$

NATO Symbol: $\mathrm{KJ}$

Chemical type: smoke
CWC schedule: $n / a$

AKA: tin tetrachloride, fuming spirit of Libavius 


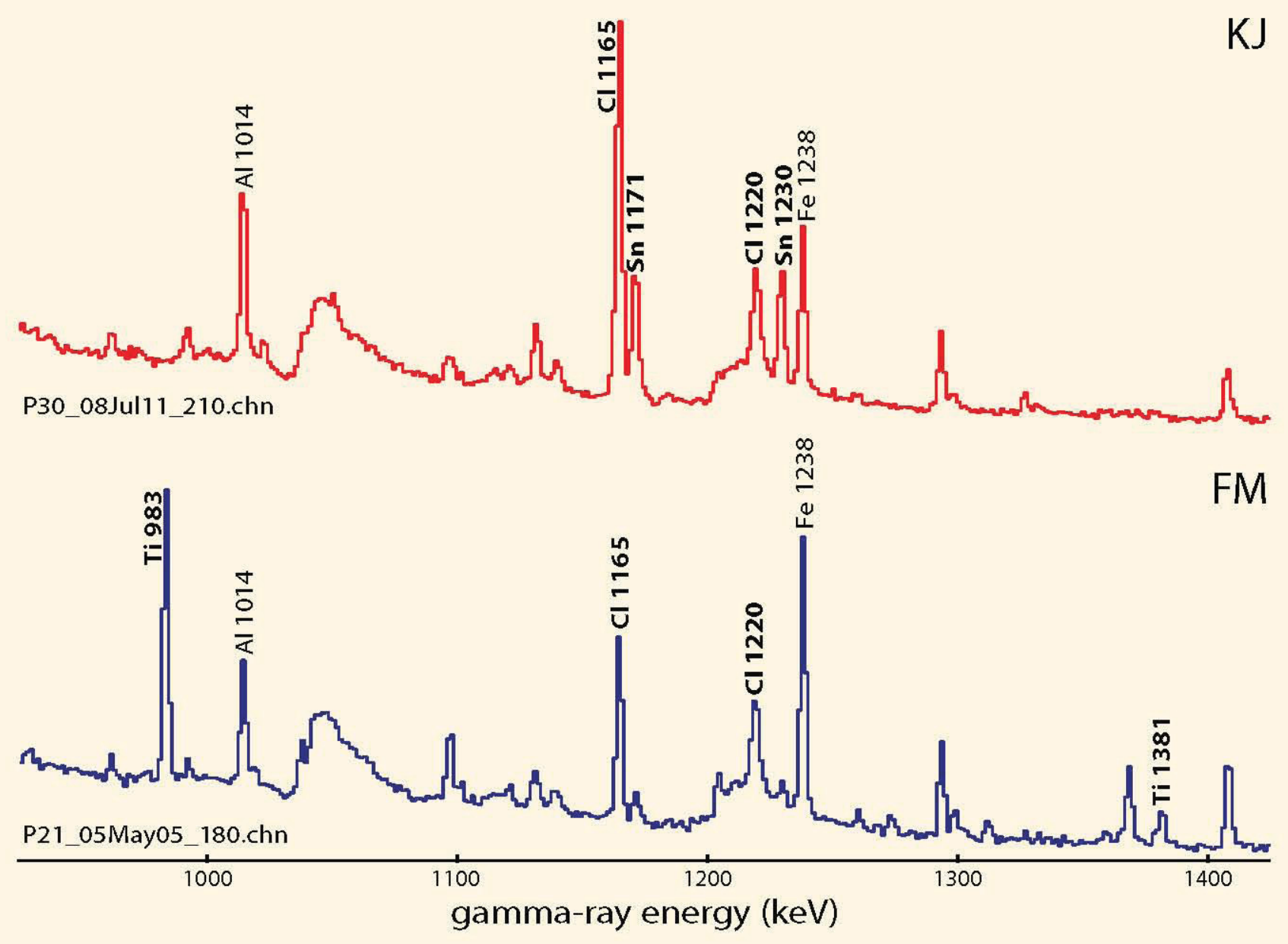

\section{Spectral comparison}

The figure above compares simulated $\mathrm{KJ}$ smoke (red curve) and actual FM smoke (blue). The KJ spectrum was measured from a 4.2-inch mortar projectile replica, and the FM spectrum from a Livens projector shell.

\section{Distinguishing features}

- KJ contains tin but no titanium.

- FM contains titanium but no tin.
Chemical name: stannic chloride

Chemical formula: $\mathrm{SnCl}_{4}$

Key elements: chlorine 54.4 wt- $\%$, tin 45.6 wt- $\%$

NATO Symbol: KJ

Chemical type: smoke
CWC schedule: $n / a$

AKA: tin tetrachloride, fuming spirit of Libavius 


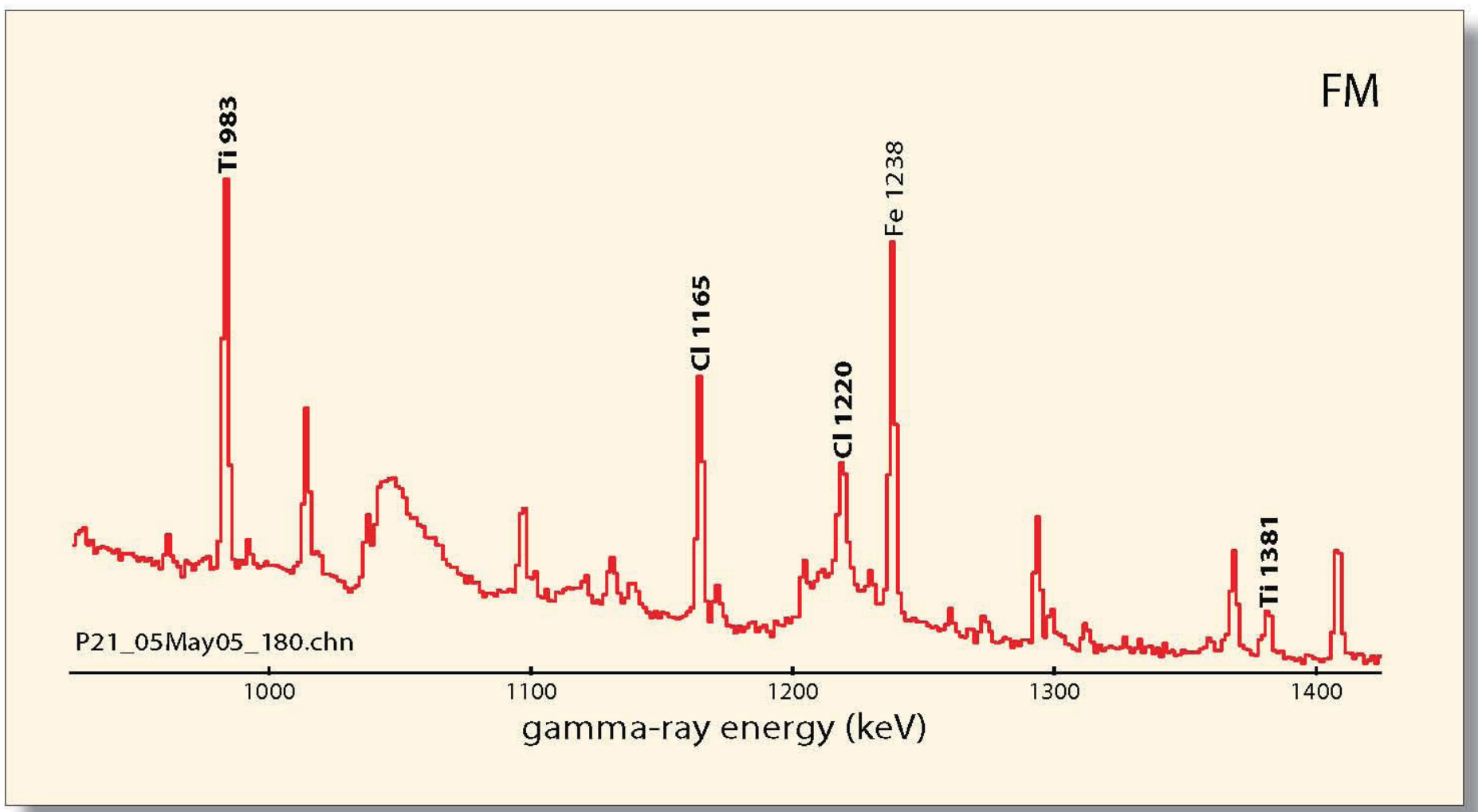

\section{Key spectral features}

- Moderate or strong chlorine peaks at 1164, 1763, 1951, and $1959 \mathrm{keV}$

- Moderate or strong titanium peaks at 983 , and $1381 \mathrm{keV}$

\section{Spectrum ID Tips}

- Chlorine peaks will be evident in the PINS spectrum within 100 seconds or less, and so will the titanium peaks.

- The 983-keV titanium peak is the strongest and clearest indicator of that element's presence.

- The moderate 2223-keV hydrogen peak is entirely due to background.

\section{Caveats}

- The hydrogen peak area can be non-zero if the background is under-subtracted.

- This spectrum was measured from a Livens projector shell.

- PINS spectra have been measured from less than a dozen FM-filled munitions. 

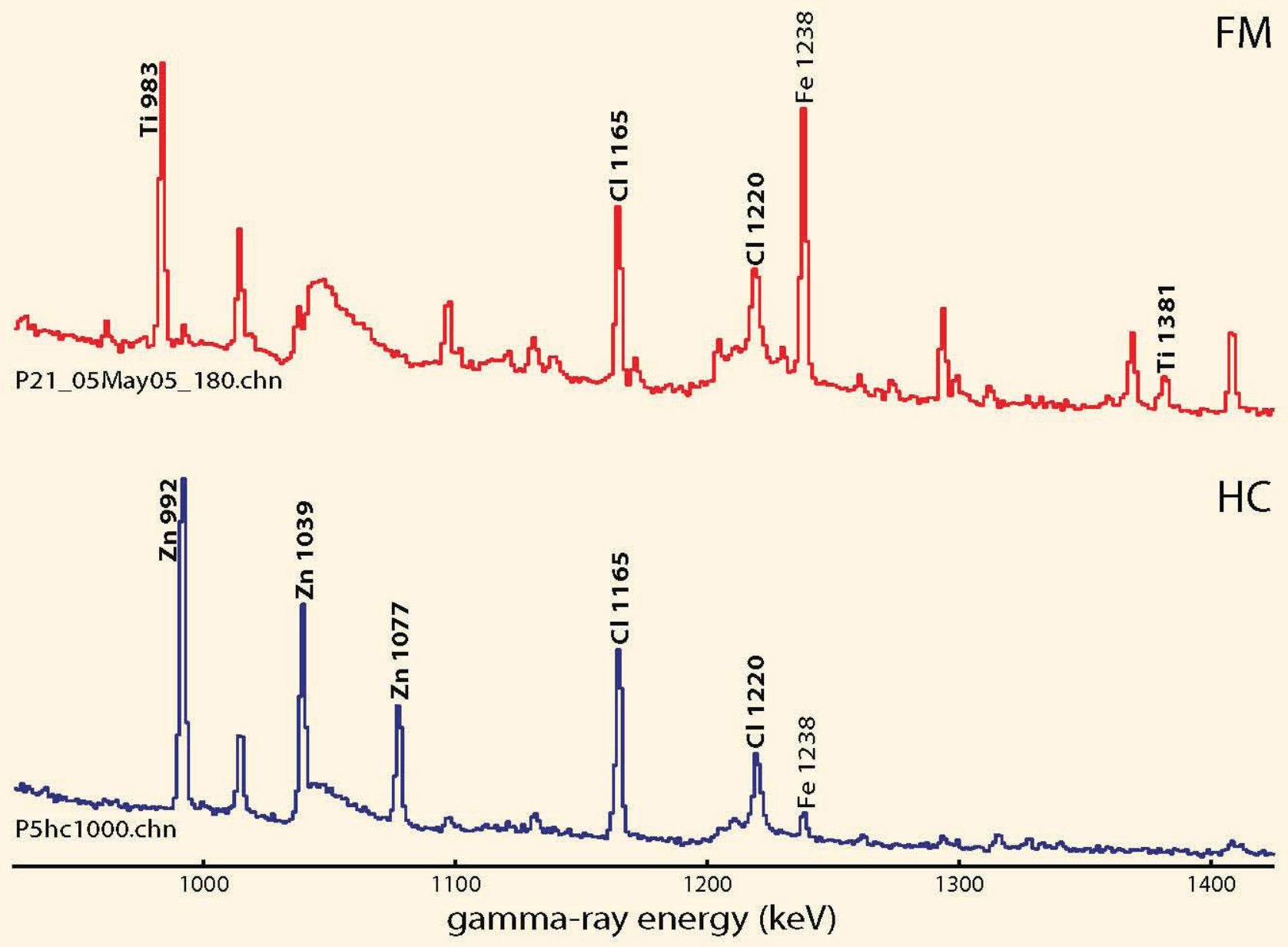

\section{Spectral comparison}

The figure above compares FM smoke (red curve) and $\mathrm{HC}$ smoke (blue). The HC spectrum was measured from a smoke pot, and the FM spectrum from a Livens projector shell.

\section{Distinguishing features}

- HC contains zinc but not titanium; FM contains titanium but not zinc.

- Look for the 983-keV peak to identify titanium, and hence FM smoke.

- The 1077-keV peak identifies zinc and hence HC smoke.

Chemical name: titanium tetrachloride

CWC schedule: $n / a$

Chemical formula: $\mathrm{TiCl}_{4}$

Key elements: chlorine 74.7 wt- $\%$, titanium 25.3 wt- $\%$

NATO Symbol: FM

Chemical type: smoke 


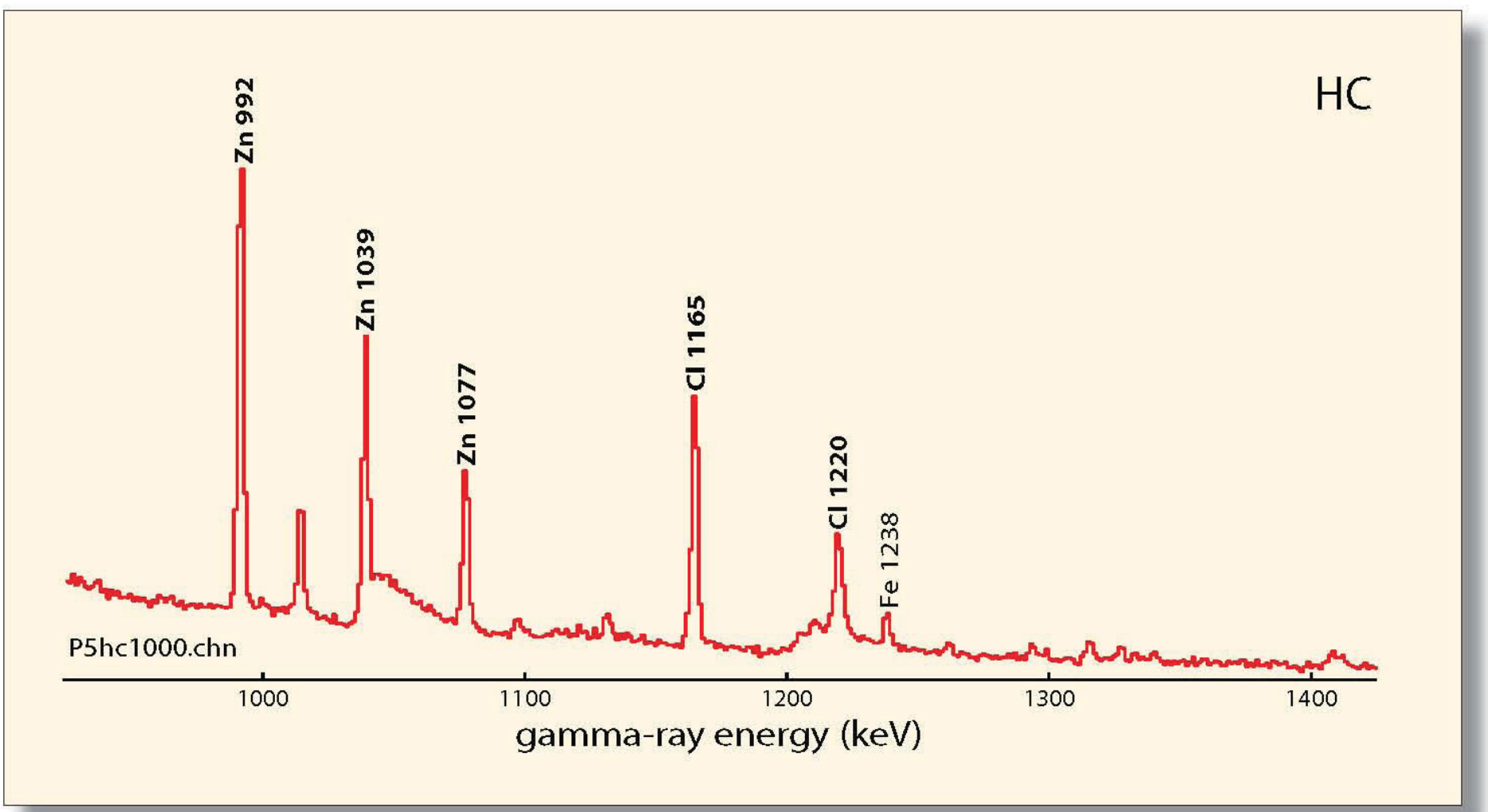

\section{Key spectral features}

- Moderate or strong chlorine peaks at 1164, 1763, 1951, and $1959 \mathrm{keV}$

- Moderate or strong zinc peaks at 991, 1039, and 1077 $\mathrm{keV}$

\section{Spectrum ID Tips}

- Chlorine peaks will be evident in the PINS spectrum within 100 seconds or less, and so will the zinc peaks.

- The 1077-keV peak is not the strongest zinc peak, but it is probably the best zinc indicator.

- The 991- and 1039-keV peaks often appear weakly as background from other elements.

- If all three peaks are evident with area ratios of approximately 3:2:1, zinc has been detected.
- The moderate 2223-keV hydrogen peak is entirely due to background.

\section{Caveats}

- The hydrogen peak area can be non-zero if the background is under-subtracted.

- This spectrum was measured from a smoke pot.
Chemical name: mixture of aluminum grains, zinc oxide, and hexachloroethane

Chemical formula: $\mathrm{Al}, 6.7 \mathrm{wt}-\% ; \mathrm{ZnO}, 46.7 \mathrm{wt}-\% ; \mathrm{C}_{6} \mathrm{Cl}_{6}, 46.7 \mathrm{wt} .-\%$

Key elements: chlorine 41.9 wt- $\%$, zinc 37.5 wt- $\%$

NATO Symbol: $\mathrm{HC}$
Chemical type: smoke

CWC schedule: $n / a$ 


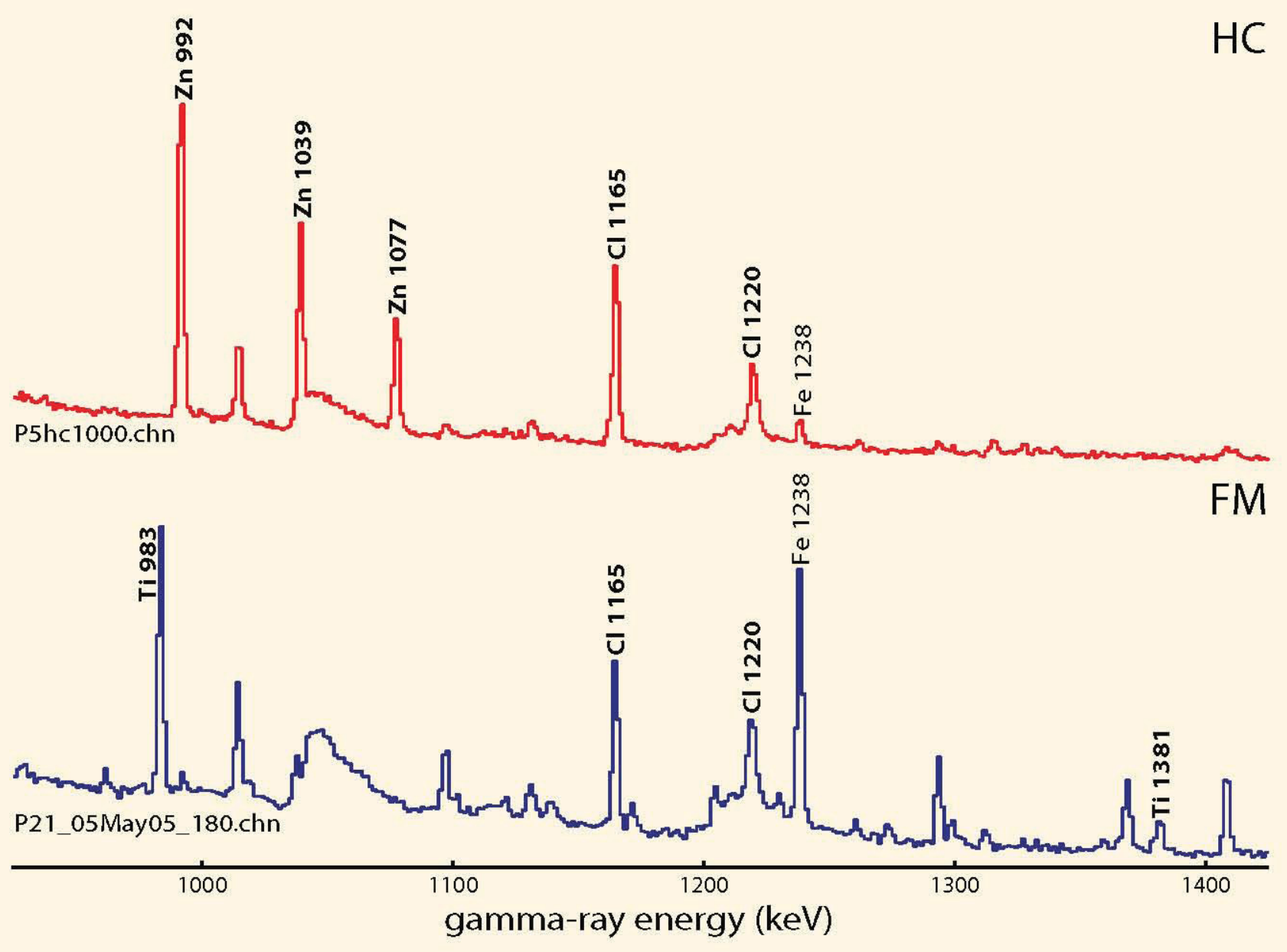

\section{Spectral comparison}

The figure above compares FM smoke (blue curve) and HC smoke (red). The $\mathrm{HC}$ spectrum was measured from a smoke pot, and the FM spectrum from a Livens projector shell.
Distinguishing features

- HC contains zinc but not titanium; FM contains titanium but not zinc.

- Look for the 983-keV peak to identify titanium, and hence FM smoke.

- The 1077-keV peak identifies zinc and hence HC smoke.
Chemical name: mixture of aluminum grains, zinc oxide, and hexachloroethane

Chemical formula: Al, $6.7 \mathrm{wt}-\% ; \mathrm{ZnO}, 46.7 \mathrm{wt}-\% ; \mathrm{C}_{6} \mathrm{Cl}_{6}, 46.7 \mathrm{wt} .-\%$

Key elements: chlorine 41.9 wt- $\%$, zinc 37.5 wt- $\%$

NATO Symbol: HC
Chemical type: smoke

CWC schedule: $n / a$ 


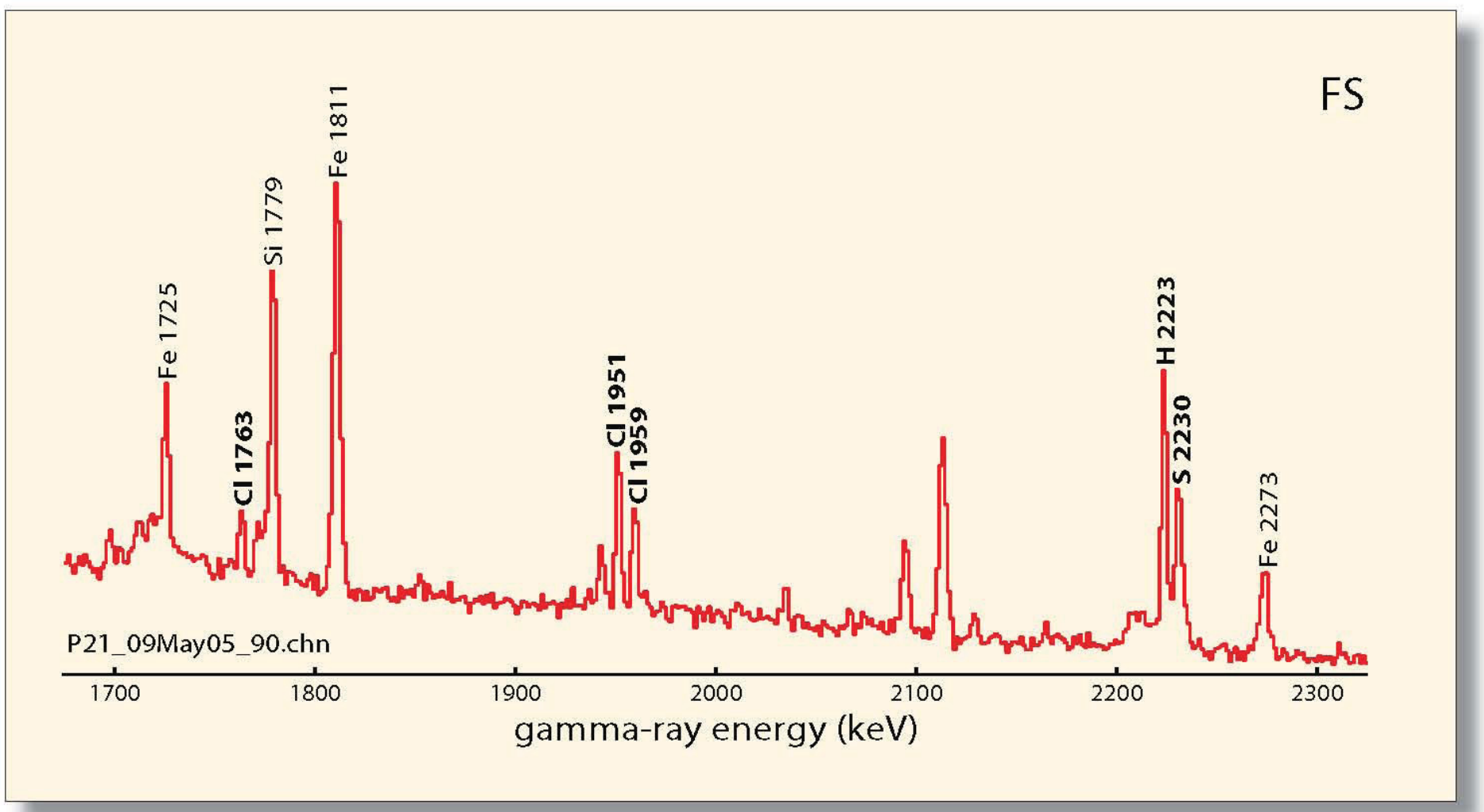

\section{Key spectral features}

- Moderate chlorine doublet peaks at 1951- and 1959-keV

- Moderate or weak hydrogen peak at 2223-keV

- Moderate sulfur peak at 2230-keV

- The 1959-keV chlorine and 2230-keV sulfur peak areas will be nearly equal .

\section{Spectrum ID Tips}

- The chlorine and sulfur peaks will be evident in 30-60 seconds.

- The sulfur peak will be roughly equal in size to the 1959-keV chlorine peak, the smaller peak on the right member of the chlorine doublet.

- Often, the hydrogen peak is smaller than the 2230-keV sulfur peak, as in the spectrum above, but it can be bigger than the sulfur peak, due to a higher background.

\section{Caveats}

- If the sulfur peak is easy to find, and it appears quickly, the fill is likely FS. Check the $\mathrm{Cl} 1959 / \mathrm{S} 2223$ ratio to make sure. If the sulfur peak is harder to find, and it takes hundreds of seconds to appear, the fill is probably mustard agent.

- Hydrogen may not be detected if the hydrogen background is over-subtracted.

- This spectrum was measured from a 4.2-inch mortar projectile.
Chemical name: sulfur trioxide dissolved in chlorosulfonic acid

Chemical formulae: $\mathrm{SO}_{3}, 55 \mathrm{wt}-\% ; \mathrm{ClSO}_{3} \mathrm{H}, 45 \mathrm{wt}-\%$

Chemical type: screening smoke

CWC schedule: $n / a$

NATO Symbol: FS
Key elements: chlorine 13.7 wt- $\%$, hydrogen 0.4 wt- $\%$, sulfur 34.4 wt- $\%$

Key element ratio: $(\mathrm{Cl} 1959) /(\mathrm{S} 2230)=1.5 \pm 1.0$ 


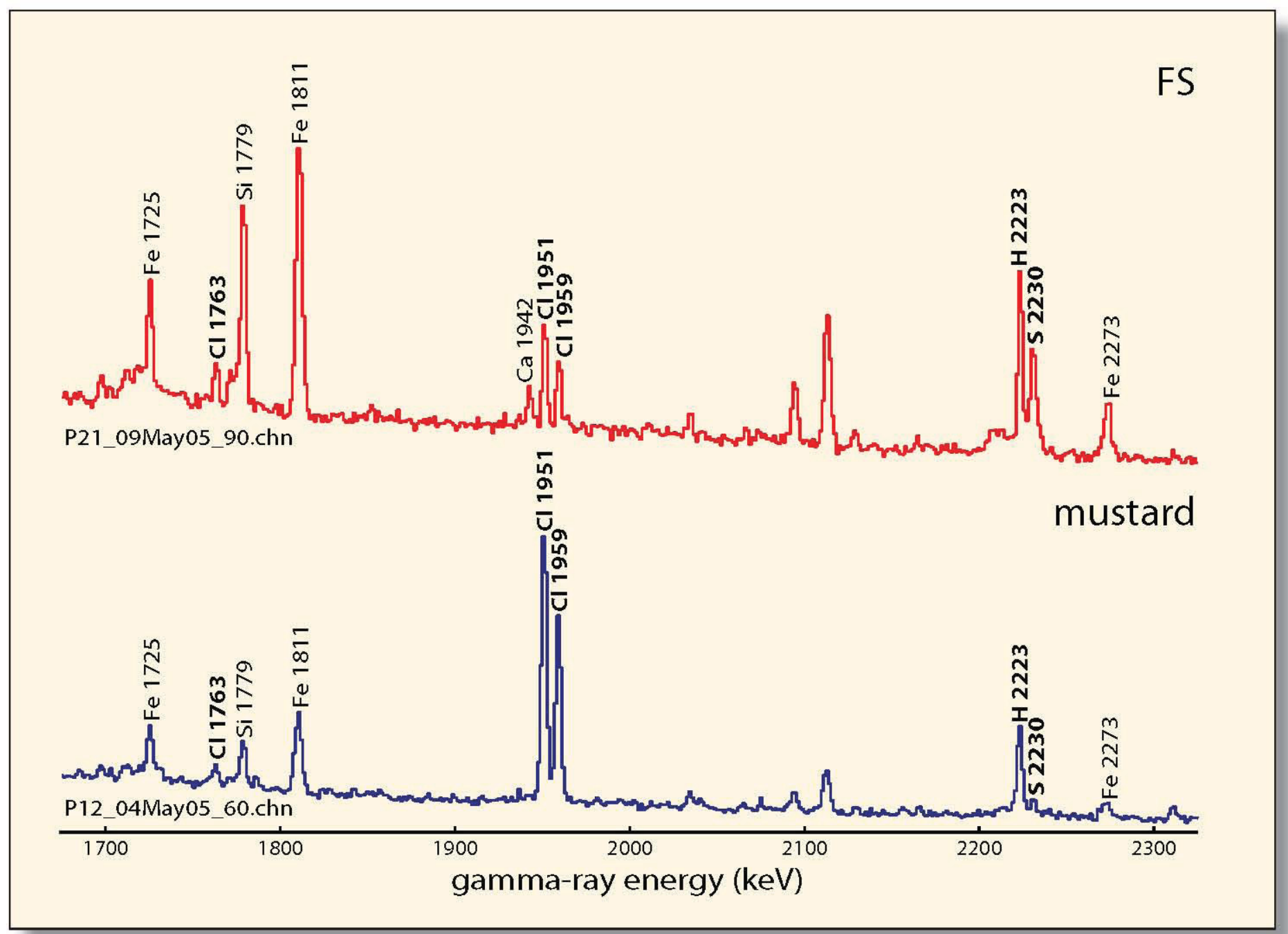

\section{Spectral comparison}

The chlorine-hydrogen spectral regions of FS smoke (red curve) and mustard agent (blue curve) are compared in the figure above. Both PINS spectra were measured from 4.2inch mortar projectiles.

\section{Distinguishing features}

- In the spectra above, the mustard agent chlorine 1959-keV to sulfur $2230-\mathrm{keV}(\mathrm{Cl} / \mathrm{S})$ peak area ratio is 12.9 , while the $\mathrm{FS}$ smoke $\mathrm{Cl} / \mathrm{S}$ ratio is almost a factor of twenty lower, 0.7 .
- The mustard agent $\mathrm{Cl} / \mathrm{S}$ ratio is rarely less than 8 or more than 16.

- The FS smoke $\mathrm{Cl} / \mathrm{S}$ ratio is usually about 1.0 , and it almost never exceeds 2.5 .

- The FS smoke hydrogen peak is mostly due to background. It is larger than the $2230-\mathrm{keV}$ sulfur peak in the spectrum above, but it sometimes appears smaller, as seen in the spectrum on the previous page.

- If the $2230-\mathrm{keV}$ sulfur peak is quickly and easily identified, the likely fill is FS smoke, not mustard agent.
Chemical name: sulfur trioxide dissolved in chlorosulfonic acid

Chemical formulae: $\mathrm{SO}_{3}, 55$ wt- $\% ; \mathrm{ClSO}_{3} \mathrm{H}, 45$ wt- $\%$

Chemical type: screening smoke

CWC schedule: $n / a$

NATO Symbol: FS
Key elements: chlorine 13.7 wt- $\%$, hydrogen 0.4 wt- $\%$, sulfur $34.4 \mathrm{wt}-\%$

Key element ratio: $(\mathrm{Cl} 1959) /(\mathrm{S} 2230)=1.5 \pm 1.0$ 


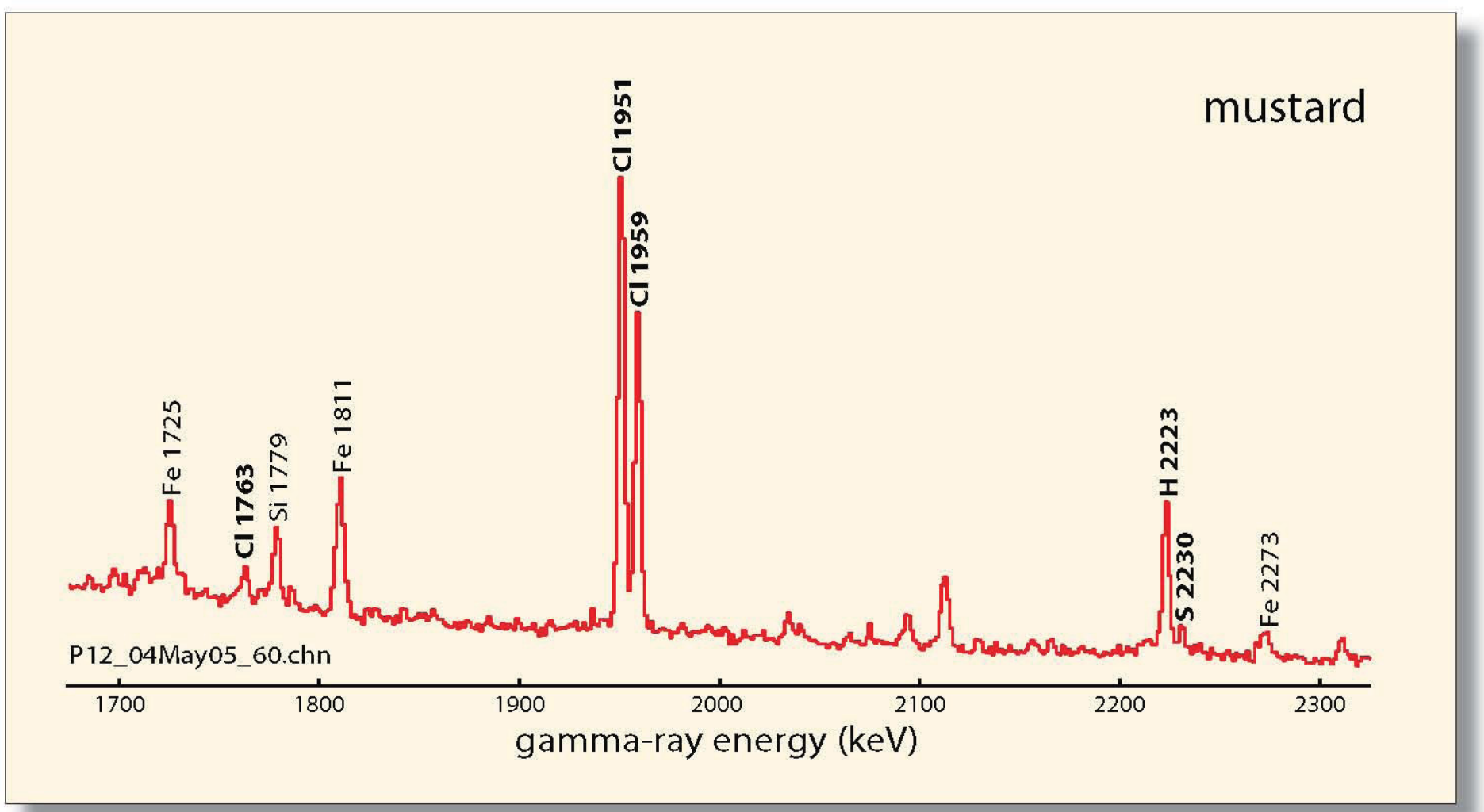

\section{Key spectral features}

- Strong chlorine doublet peaks at 1951- and 1959-keV

- Moderate hydrogen peak at 2223-keV

- Weak sulfur peak at 2230-keV

- The 1959-keV chlorine peak will appear about ten times bigger than the 2230-keV sulfur peak.

\section{Spectrum ID Tips}

- The chlorine and hydrogen peaks will be evident in 30 seconds.

- The sulfur peak may take 300-500 seconds to appear.

- The sulfur peak will be roughly a factor of ten smaller than the 1959-keV chlorine peak.

- Note the comparison of mustard and FS smoke spectra on the next page.

\section{Caveats}

- The hydrogen peak amplitude is normally smaller than the chlorine doublet peaks, as in the figure above, but it may appear bigger, depending on the background hydrogen level.

- Two mustard agent variants, $\mathrm{H}$ (also called HS) and $H D$, differ principally by the impurities they contain.

- Agent HT is a mixture of agent HD, $60 \%$ and agent $\mathrm{T}, 40 \%$.

- Agent T, bis\{2(2-chloroethylthio)ethyl\} ether, is a chlorine-sulfur compound related to HD.

- Since agents $H, H D$, and HT have similar elemental ratios, PINS does not distinguish between them.

- This spectrum was measured from a 4.2-inch mortar projectile
Chemical name: bis (2-chloroethyl) sulfide

Chemical formula: $\mathrm{C}_{4} \mathrm{H}_{8} \mathrm{Cl}_{2} \mathrm{~S}$

Key elements: Cl 44.7 wt- $\%$, H 5.0 wt- $\%$, S 20.1 wt- $\%$

Key element ratio: $(\mathrm{Cl} 1959) /(\mathrm{S} 2230)=12 \pm 4$

NATO Symbols: Hor HS, HD
Chemical type: blister agent

CWC schedule: 1

AKA: mustard gas, das Löst, Yperite 

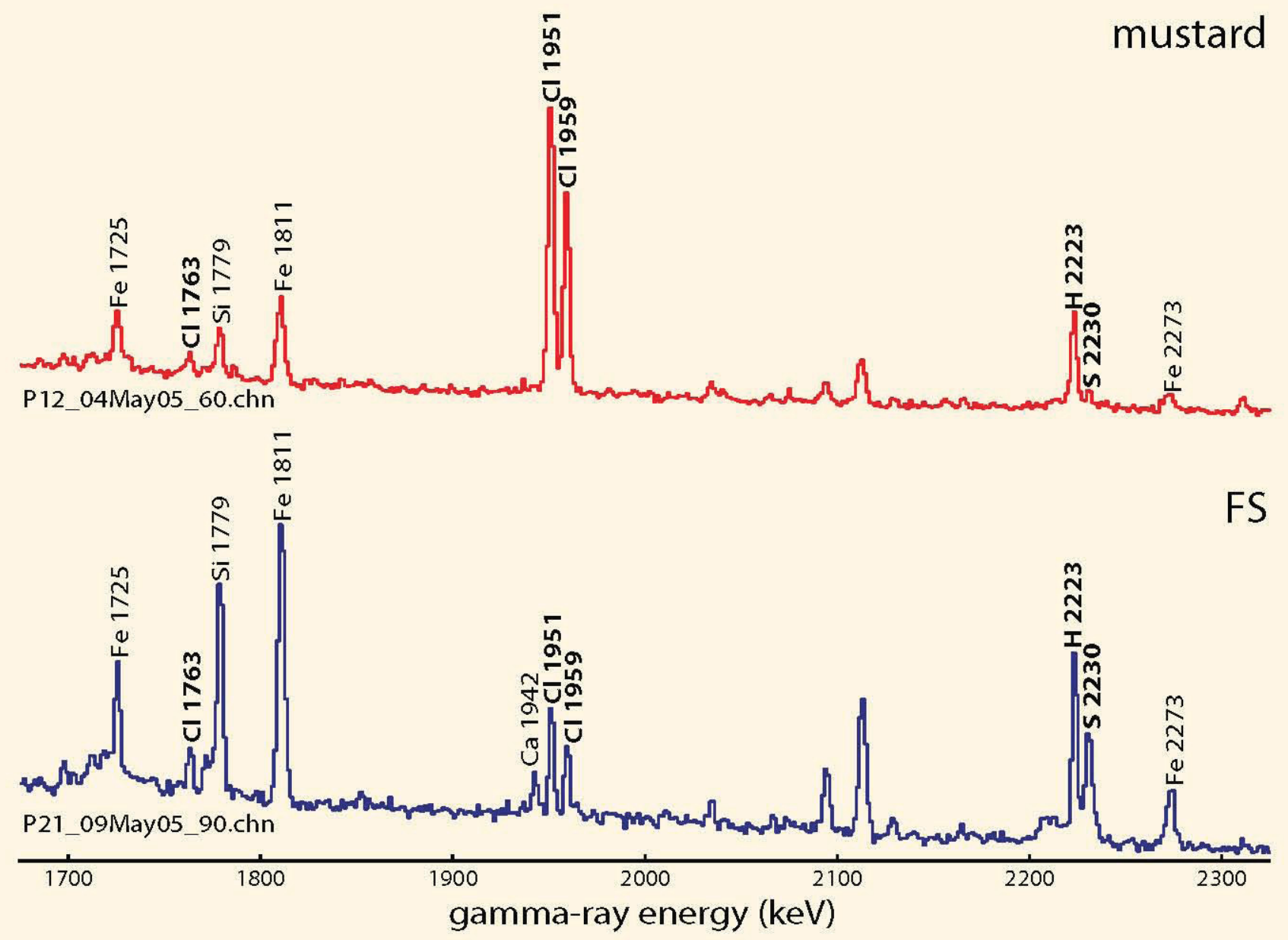

\section{Spectral comparison}

PINS spectra of mustard agent (red curve) and FS smoke (blue curve) are compared in the figure above. Both PINS spectra were recorded from 4.2-inch mortar projectiles.

\section{Distinguishing features}

- In the spectra above, the mustard agent chlorine 1959-keV to sulfur $2230-\mathrm{keV}(\mathrm{Cl} / \mathrm{S})$ peak area ratio is 12.9 , while the $\mathrm{FS}$ smoke $\mathrm{Cl} / \mathrm{S}$ ratio is almost a factor of twenty lower, 0.7 .
- The mustard agent $\mathrm{Cl} / \mathrm{S}$ ratio is rarely less than 8 or more than 16.

- The FS smoke $\mathrm{Cl} / \mathrm{S}$ ratio is usually about 1.0 , and it almost never exceeds 2 .

- The FS smoke hydrogen peak is mainly background, and it can appear smaller than the $2230-k e V$ sulfur peak.

- If the 2230-keV sulfur peak is quickly and easily identified, the likely fill is FS smoke, not mustard agent.
Chemical name: bis (2-chloroethyl) sulfide

Chemical formula: $\mathrm{C}_{4} \mathrm{H}_{8} \mathrm{Cl}_{2} \mathrm{~S}$

Key elements: Cl 44.7 wt- $\%, \mathrm{H} 5.0$ wt-\%, S 20.1 wt-\%

Key element ratio: $(\mathrm{Cl} 1959) /(\mathrm{S} 2230)=12 \pm 4$

NATO Symbols: $\mathrm{H}$ or HS, HD
Chemical type: blister agent

CWC schedule: 1

AKA: mustard gas, das Löst, Yperite 


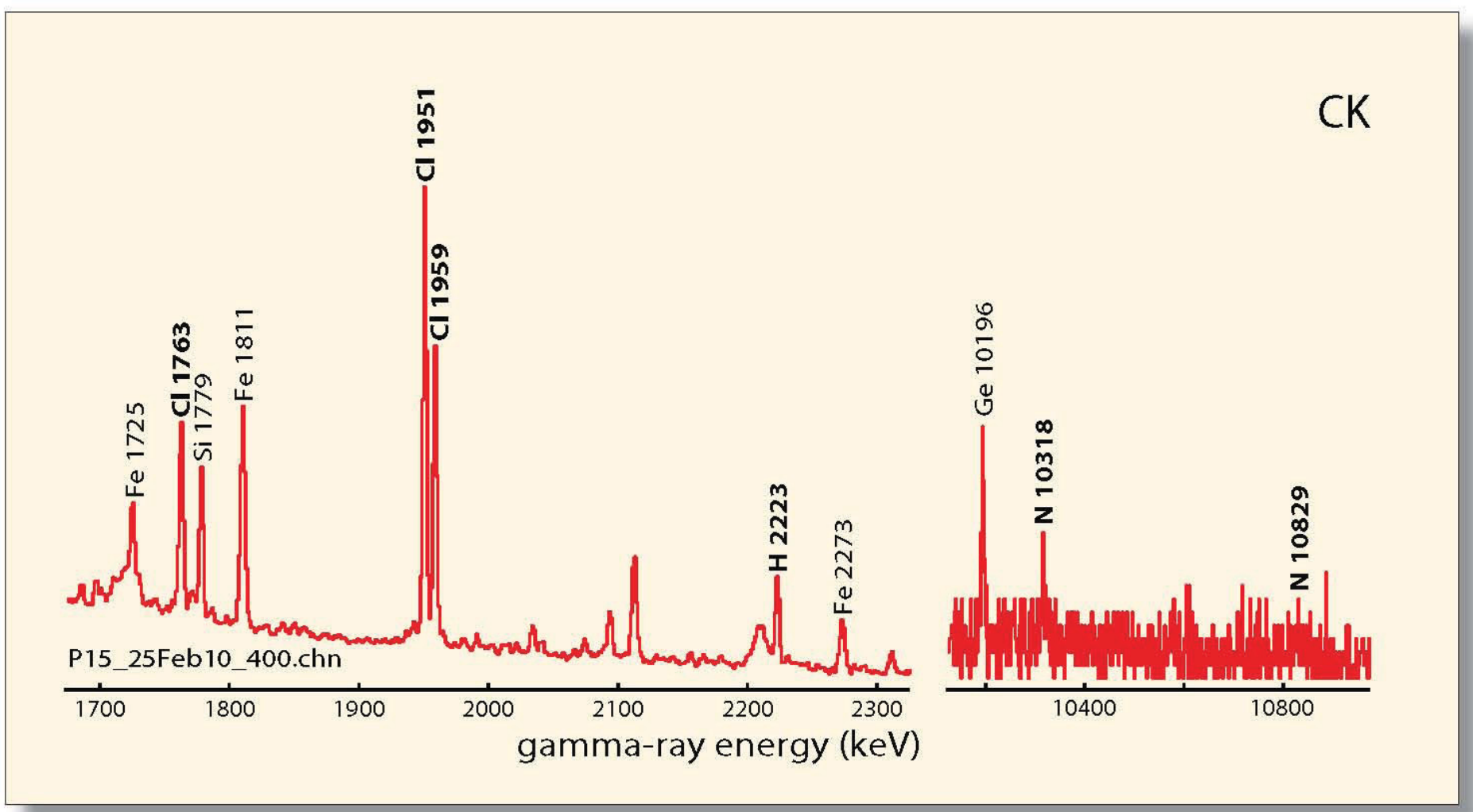

\section{Key spectral features}

- Moderate or strong chlorine peaks at 1763, 1951, and $1959 \mathrm{keV}$

- Weak nitrogen peaks at $10318,10829 \mathrm{keV}$

- Only background-level hydrogen peak

\section{Spectrum ID Tips}

- Chlorine peaks will be evident in the PINS spectrum within 100 seconds or less.

- The 1763-keV Cl inelastic peak is approximately equal to the 1959-keV Cl capture peak.

- The 2223-keV hydrogen peak is entirely due to background.

- Weak nitrogen peaks are also observed, typically after 1,000 seconds of counting.

\section{Caveats}

- The indicated hydrogen signal may be non-zero due to background under-subtraction.

- Be sure to continue the assay for at least 1,000 seconds, to identify the presence of nitrogen.

- CK may contain sodium pyrophosphate at the $5 \%$ level, to stabilize the agent against a potentially explosive polymerization reaction. PINS may detect phosphorus in this instance, in addition to chlorine and nitrogen.

- This spectrum was measured from simulated CK in a 4.2-inch mortar projectile replica.

- No PINS spectra have been measured from containers or munitions filled with agent CK.
Chemical name: cyanogen chloride

\section{Chemical formula: $\mathrm{CNCl}$}

Key elements: chlorine 22.8 wt- $\%$, nitrogen 5.0 wt- $\%$

Key element ratios: $(\mathrm{Cl} 1763) /(\mathrm{Cl} 1959)=1.0 \pm 0.3$
NATO Symbol: CK

Chemical type: blood agent

CWC schedule: 3 

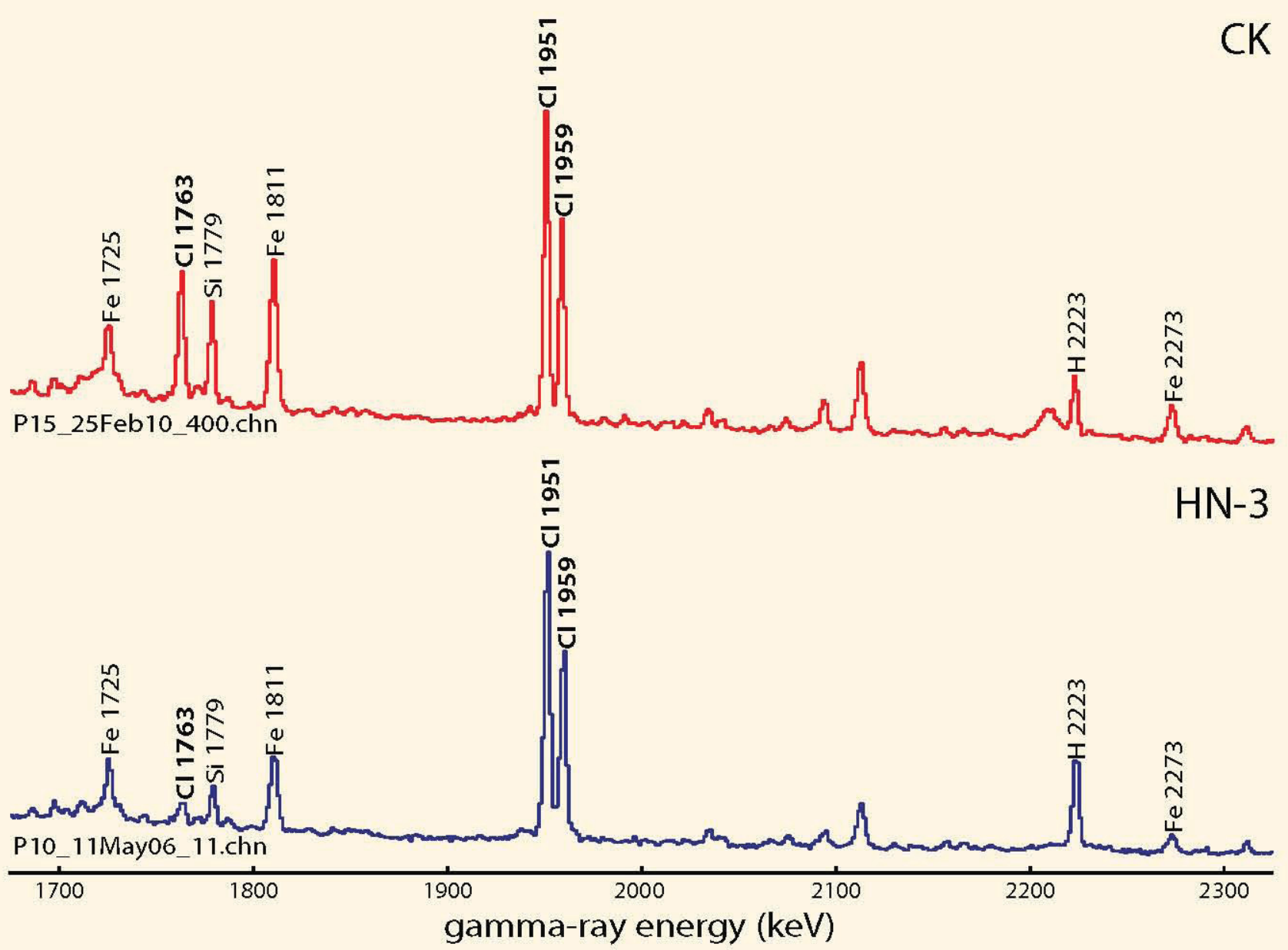

\section{Spectral comparison}

The figure above compares simulated CK (red curve) and HN-3 (blue). The CK spectrum was measured from a 4.2-inch mortar projectile replica, and the $\mathrm{HN}-3$ spectrum from a German Traktor rocket.

\section{Distinguishing features}

- The chlorine inelastic-to-capture (Cl 1763/Cl 1959) peak ratio is approximately 1.0 for $\mathrm{CK}$, but the ratio is less than 0.1 for $\mathrm{HN}-3$.
Chemical name: cyanogen chloride

Chemical formula: $\mathrm{CNCl}$

Key elements: chlorine 22.8 wt- $\%$, nitrogen 5.0 wt- $\%$

Key element ratios: $(\mathrm{Cl} 1763) /(\mathrm{Cl} 1959)=1.0 \pm 0.3$
NATO Symbol: CK

Chemical type: blood agent

CWC schedule: 3 


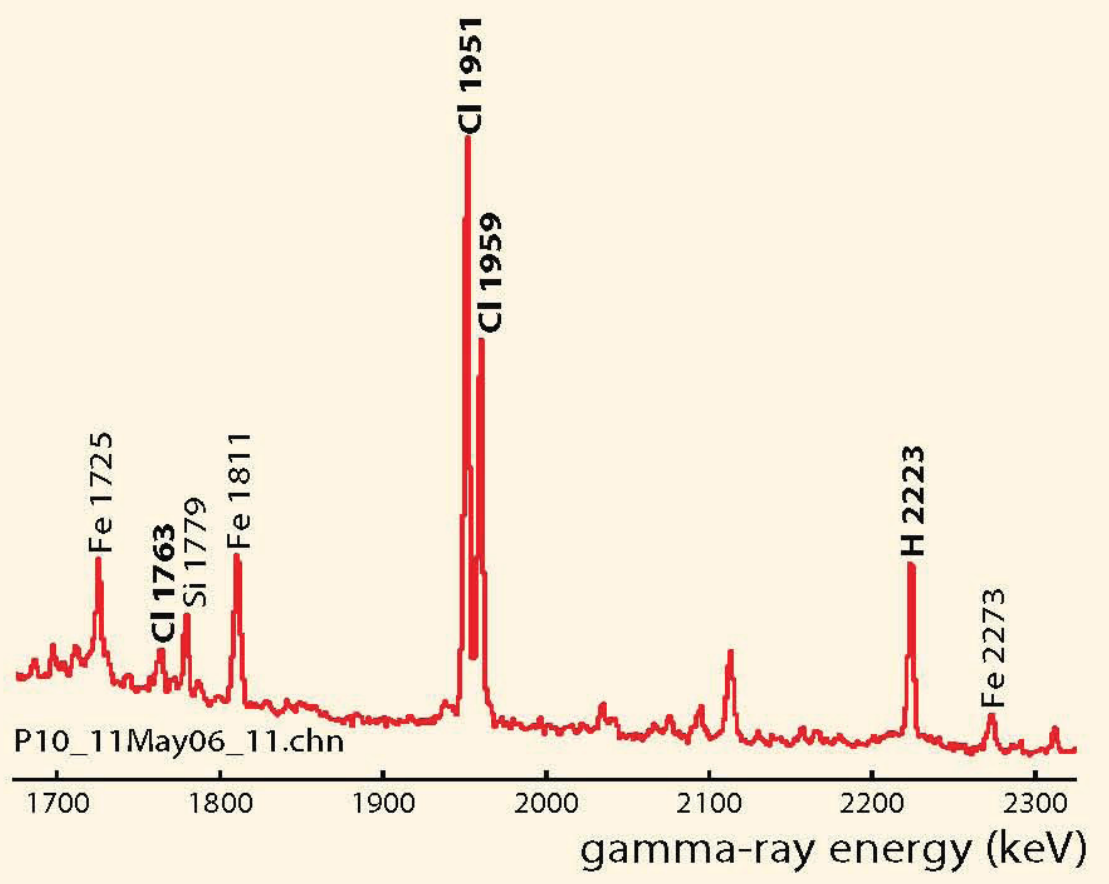

\section{$\mathrm{HN}-3$}

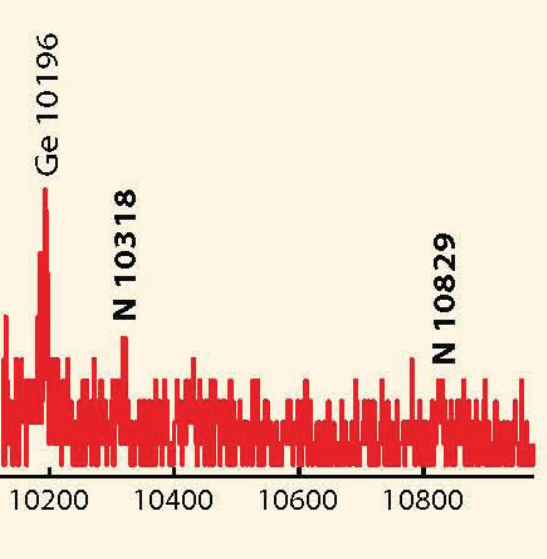

\section{Key spectral features}

- Moderate or strong chlorine peaks at 1164, 1763, 1951, and $1959 \mathrm{keV}$

- Weak nitrogen peaks at $10318,10829 \mathrm{keV}$

- Strong hydrogen peak

\section{Spectrum ID Tips}

- Chlorine peaks will be evident in the PINS spectrum within 100 seconds or less.

- The 1763-keV Cl inelastic peak is weaker than the 1959-keV Cl capture peak.

- The 2223-keV hydrogen peak is strong, but the chlorine peaks at 1951 and $1959 \mathrm{keV}$ will be three times as strong.

- Weak nitrogen peaks will be observed in 1,000 seconds or so.

\section{Caveats}

- Be sure to continue the assay for at least 1,000 seconds, to identify the presence of nitrogen.

- The chemical formula below is for the nitrogen mustard-3 variant, but the nitrogen mustard- 1 and nitrogen mustard-2 variants are similar. Nitrogen mustard -3 is the most stable of the three variants, and it is one of the chemical fills of World War II German Traktor rockets.

- The spectrum was measured from a German Traktor rocket.
Chemical name: 2, 2; 2"-Trichlorotriethylamine

Chemical formula: $\mathrm{C}_{6} \mathrm{H}_{12} \mathrm{Cl}_{3} \mathrm{~N}$

Key elements: chlorine 22.8 wt- $\%$, nitrogen 5.0 wt- $\%$

Key element ratios: $(\mathrm{Cl} 1959) /(\mathrm{H} 2223)=3.5 \pm 0.4$

$(\mathrm{Cl} 1763) /(\mathrm{Cl} 1959)=0.16 \pm 0.15$
NATO Symbol: $\mathrm{HN}-3$

Chemical type: blister agent

CWC schedule: 1

AKA: nitrogen mustard - 3 

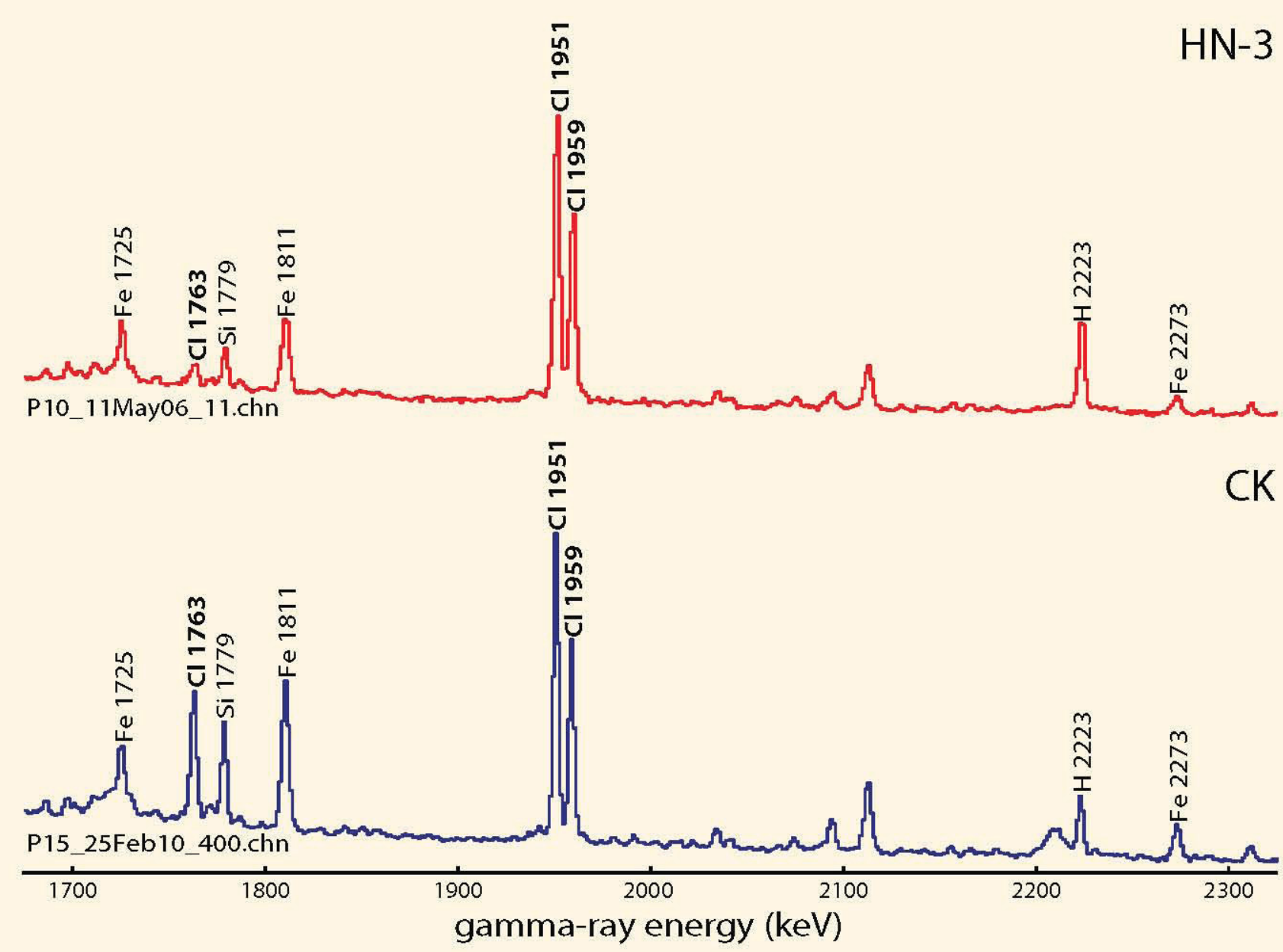

\section{Spectral comparison}

The figure above compares simulated CK (blue curve) and $\mathrm{HN}-3$ (red). The CK spectrum was measured from a 4.2-inch mortar projectile replica, and the $\mathrm{HN}-3$ spectrum from a German Traktor rocket.

\section{Distinguishing features}

- The chlorine inelastic-to-capture (Cl 1763/Cl 1959) peak ratio is greater than 1.0 for $\mathrm{CK}$, but the ratio is less than 0.2 for $\mathrm{HN}-3$.
Chemical name: 2, 2, 2"-Trichlorotriethylamine

Chemical formula: $\mathrm{C}_{6} \mathrm{H}_{12} \mathrm{Cl}_{3} \mathrm{~N}$

Key elements: chlorine $22.8 \mathrm{wt}-\%$, nitrogen $5.0 \mathrm{wt}-\%$

Key element ratios: $(\mathrm{Cl} 1959) /(\mathrm{H} 2223)=3.5 \pm 0.4$

$(\mathrm{Cl} 1763) /(\mathrm{Cl} 1959)=0.16 \pm 0.15$
NATO Symbol: HN-3

Chemical type: blister agent

CWC schedule: 1

AKA: nitrogen mustard - 3 


\section{Chapter 8: \\ Bromine-Based Chemical Identification}

While there are multiple toxic bromine compounds, just one - bromobenzyl cyanide - has been militarized by the United States. Bromobenzyl cyanide is called agent CA for short. This tear-producing agent was used during World War I, but it was replaced in the 1920s by riot agents based on chloroacetophenone, agent $\mathrm{CN}$.[1]

\section{Riot Agent CA}

Agent CA is one of the most powerful tear-producing agents. [2] In addition to bromine, agent CA contains the PINS key elements hydrogen and nitrogen.

\section{Spectral Identification}

As shown in Figure 8-1, identification of agent CA requires detection of bromine, hydrogen, and nitrogen gamma-ray peaks. The strongest bromine gamma ray is the peak at $276 \mathrm{keV}$.

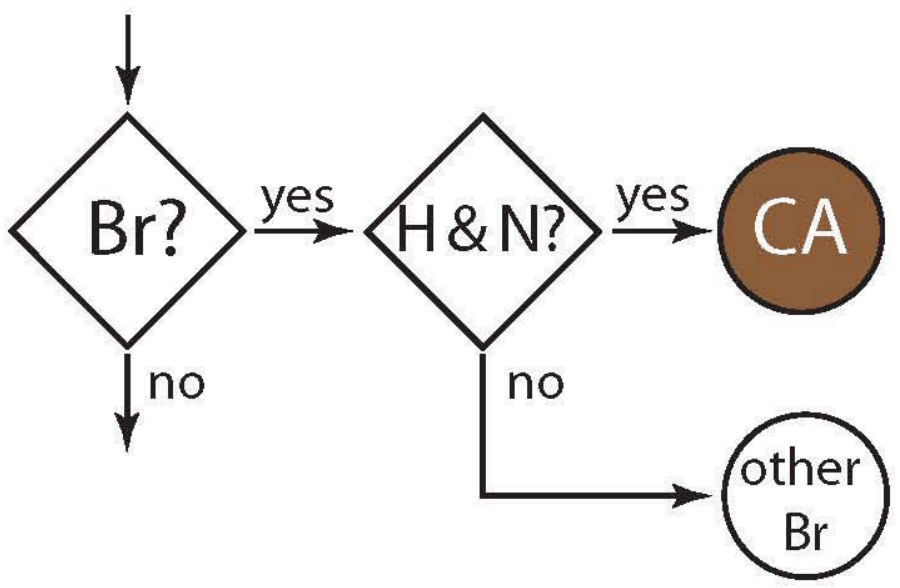

Figure 8-1: Bromine chemical identification branch of the PINS decision tree.

\section{References}

1. James A.F. Compton, Military Chemical and Biological Agents (Caldwell, NJ: The Telford Press, 1987) pages 209 to 214. Military Chemistry and Chemical Compounds, U.S. Army Field Manual 3-9, October 1975, pages 3-23 and 3-24.

2. A.M. Prentiss, Chemicals in War (New York: McGraw-Hill, 1937) page 144.

\section{riot agent}


74 PINS Spectrum Identification Guide 


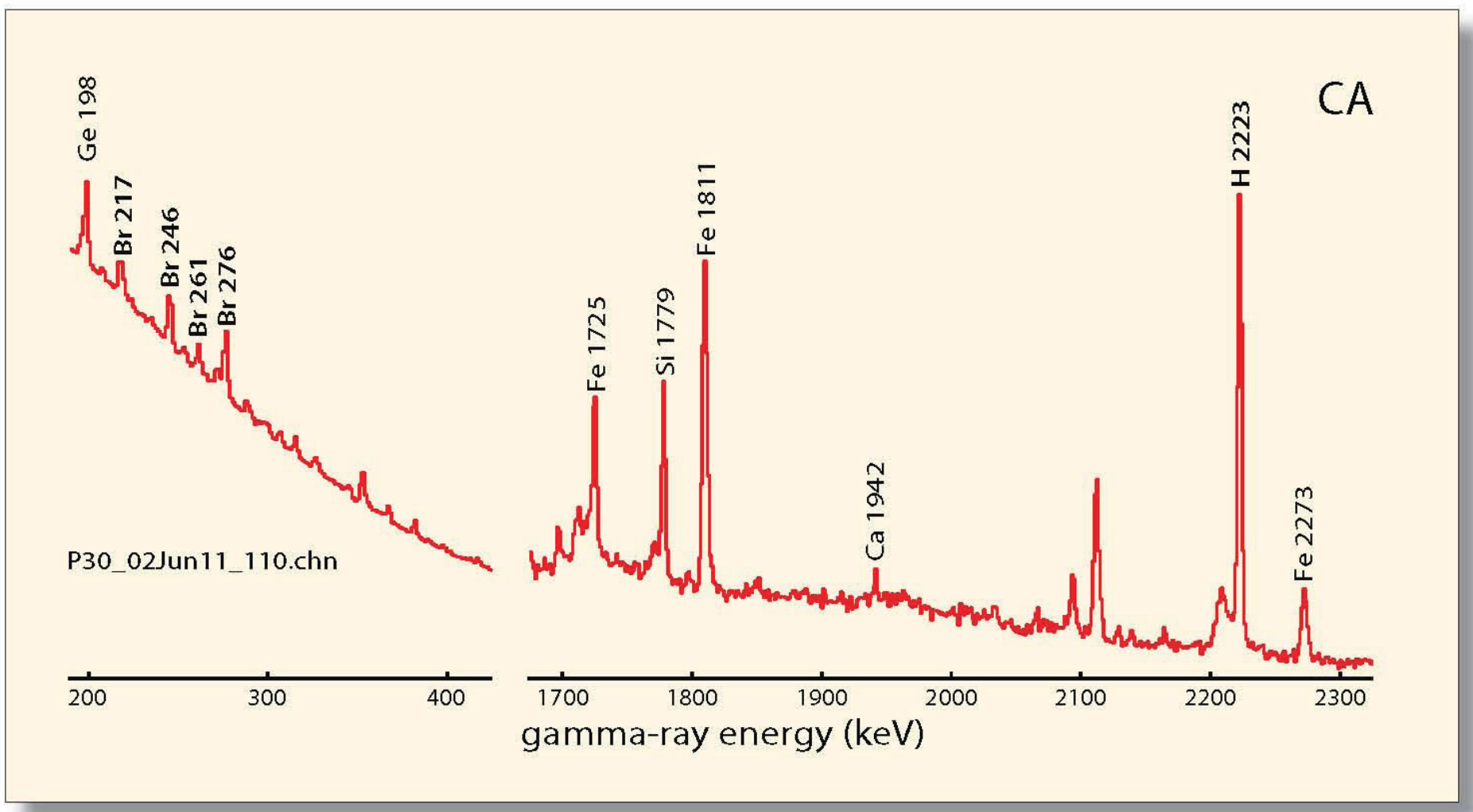

\section{Key spectral features}

- Moderate or strong bromine peaks at 217, 245, 261, and $276 \mathrm{keV}$

- Moderate or strong hydrogen peak at $2223 \mathrm{keV}$

- Weak nitrogen peak(s) at 10318 and/or $10829 \mathrm{keV}$

\section{Spectrum ID Tips}

- Bromine peaks will be evident in the PINS spectrum within 100 seconds or less, and so will the hydrogen peak.

- The nitrogen peak(s) may take over 1,000 seconds to become visible.

\section{Caveats}

- A 3,000 second counting interval may be necessary to observe the nitrogen peak(s) from a small munition like a 75-mm artillery projectile.

- This spectrum was measured from a 4.2 inch mortar projectile replica containing simulated agent $C A$.

- No PINS spectra have been measured from agent CA-filled containers or munitions.
Chemical name: bromobenzyl cyanide

Chemical formula: $\mathrm{C}_{8} \mathrm{H}_{6} \mathrm{BrN}$

Key elements: hydrogen 3.0 wt.- $\%$, bromine 40.8 wt. $-\%$, nitrogen 7 wt. $-\%$
Chemical type: riot agent

CWC schedule: $n / a$

AKA: BBC, camite

NATO Symbol: CA 
76 PINS Spectrum Identification Guide 


\section{Chapter 9: \\ Nitrogen-Based Chemical Identification}

\section{Explosive Identification}

The nitrogen-based chemicals identified by PINS are explosives. Military explosives contain the elements carbon, hydrogen, nitrogen, and oxygen. Table 9-1 displays the elemental composition of several common military explosives.

\begin{tabular}{llcccc}
\hline \multicolumn{6}{l}{ Table 9-1: Elemental Composition of Explosives, in Weight-\%. } \\
\hline Explosive & Formula* $^{*}$ & carbon & hydrogen & nitrogen & oxygen \\
Comp. B & RDX 60\%, TNT 40\% & 24.5 & 2.5 & 30.4 & 42.8 \\
C-4 & $\mathrm{RDX} 90 \%$, PIB** $10 \%$ & 23.2 & 3.9 & 34.1 & 38.9 \\
$\mathrm{HMX}$ & $\mathrm{C}_{4} \mathrm{H}_{8} \mathrm{~N}_{8} \mathrm{O}_{8}$ & 16.2 & 2.7 & 38.7 & 43.2 \\
PETN & $\mathrm{C}_{5} \mathrm{H}_{8} \mathrm{~N}_{4} \mathrm{O}_{12}$ & 19.0 & 2.5 & 17.7 & 60.8 \\
RDX & $\mathrm{C}_{3} \mathrm{H}_{6} \mathrm{~N}_{6} \mathrm{O}_{6}$ & 16.2 & 2.7 & 37.8 & 43.2 \\
TNT & $\mathrm{C}_{7} \mathrm{H}_{5} \mathrm{~N}_{3} \mathrm{O}_{6}$ & 37.0 & 2.2 & 18.5 & 42.3 \\
\hline
\end{tabular}

${ }^{*}$ Chemical formulae from reference 1 .

${ }^{* *}$ polyisobutylene (PIB), $\mathrm{C}_{4} \mathrm{H}_{8}[2]$.

However, the PINS Cf-252 source does not excite carbon or oxygen very well, as discussed in Chapter 1 . Hence the PINS explosive identification logic reduces to detection of the key elements nitrogen and hydrogen, as shown in Figure 9-1. As noted in previous chapters, hydrogen gamma-ray detection is performed by simply fitting a Gaussian to the peak at $2223 \mathrm{keV}$ and evaluating its area and uncertainty. Nitrogen gamma-ray peak analysis, the principal topic of this chapter, is much more challenging, and yet it defines the critical path for PINS explosive identification.

\section{Nitrogen Gamma-Ray Peak Analysis}

The PINS nitrogen peaks are rather weak in intensity, for three reasons: the nitrogen thermal capture cross section is just 80 millibarns, the branching ratio for the N $10829-\mathrm{keV}$ peak is low, 0.14 , and the HPGe detector's efficiency is an order of magnitude lower at $10 \mathrm{MeV}$ than at $2 \mathrm{MeV}$. Two nitrogen spectral lines are of interest, at $10318 \mathrm{keV}$ and $10829 \mathrm{keV}$. The higher energy peak is produced by the neutron capture reaction ${ }^{14} \mathrm{~N}+\mathrm{n} \rightarrow{ }^{15} \mathrm{~N}^{*} \rightarrow{ }^{15} \mathrm{~N}+\gamma$, while the lower energy peak is the corresponding single-escape peak. Fortunately, the background is rather low in this spectral region.
The nitrogen peaks cannot be fit with Gaussians, as they contain too few counts for a reasonable counting time. Hence, PINS nitrogen peaks are analyzed using the summing methods described below.

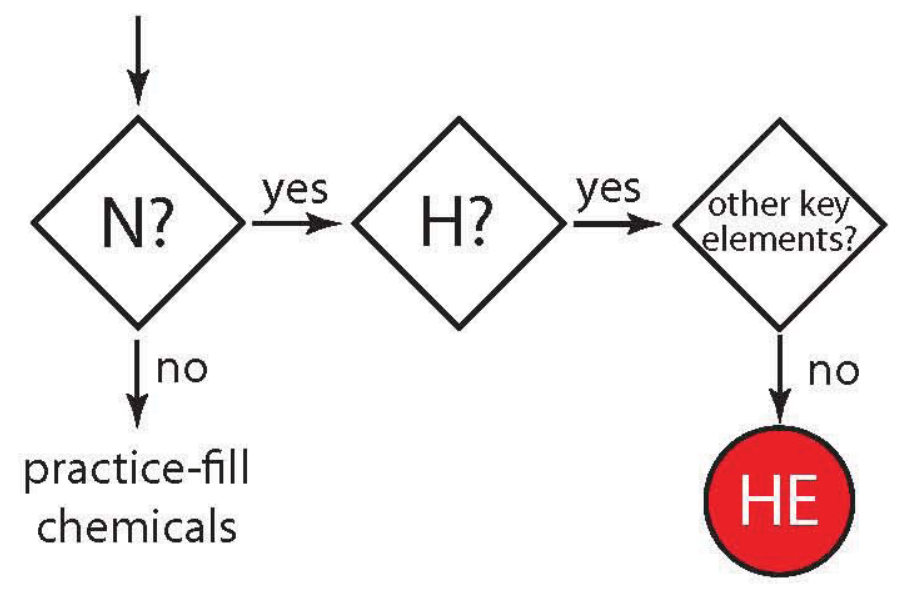

Figure 9-1. PINS Explosive Identification Logic. 
The test for nitrogen detection is based on the following characteristics of typical PINS spectra in the high-energy, nitrogen gamma-ray region:

- The background count is essentially constant across the region containing possible nitrogen peaks, and the expected number of background counts per channel is small. (In fact there will be many channels with no background counts recorded.)

- For the purpose of nitrogen detection, counts in the 9-channel regions centered at $10318 \mathrm{keV}$ and 10829 $\mathrm{keV}$ are considered to be possible nitrogen peak counts. Counts in 40-channel regions to either side of the 9-channel peak regions are used to represent the background. Between each 9-channel peak region and 40-channel background region is a 9-channel "buffer" region, that is not used in the calculations. The buffer regions account for the possibility of the peak regions not being well-centered at the $10318 \mathrm{keV}$ and 10829 $\mathrm{keV}$ energies due to energy calibration errors. The summing regions for the $10318 \mathrm{keV}$ nitrogen singleescape peak are shown in Figure 9-2 below.

- If there is no nitrogen present in the sample under test, then the only counts that occur in the nitrogen peak channels will be background counts and they will occur with the same probability (i.e., at the same rate) as counts in the background channels.

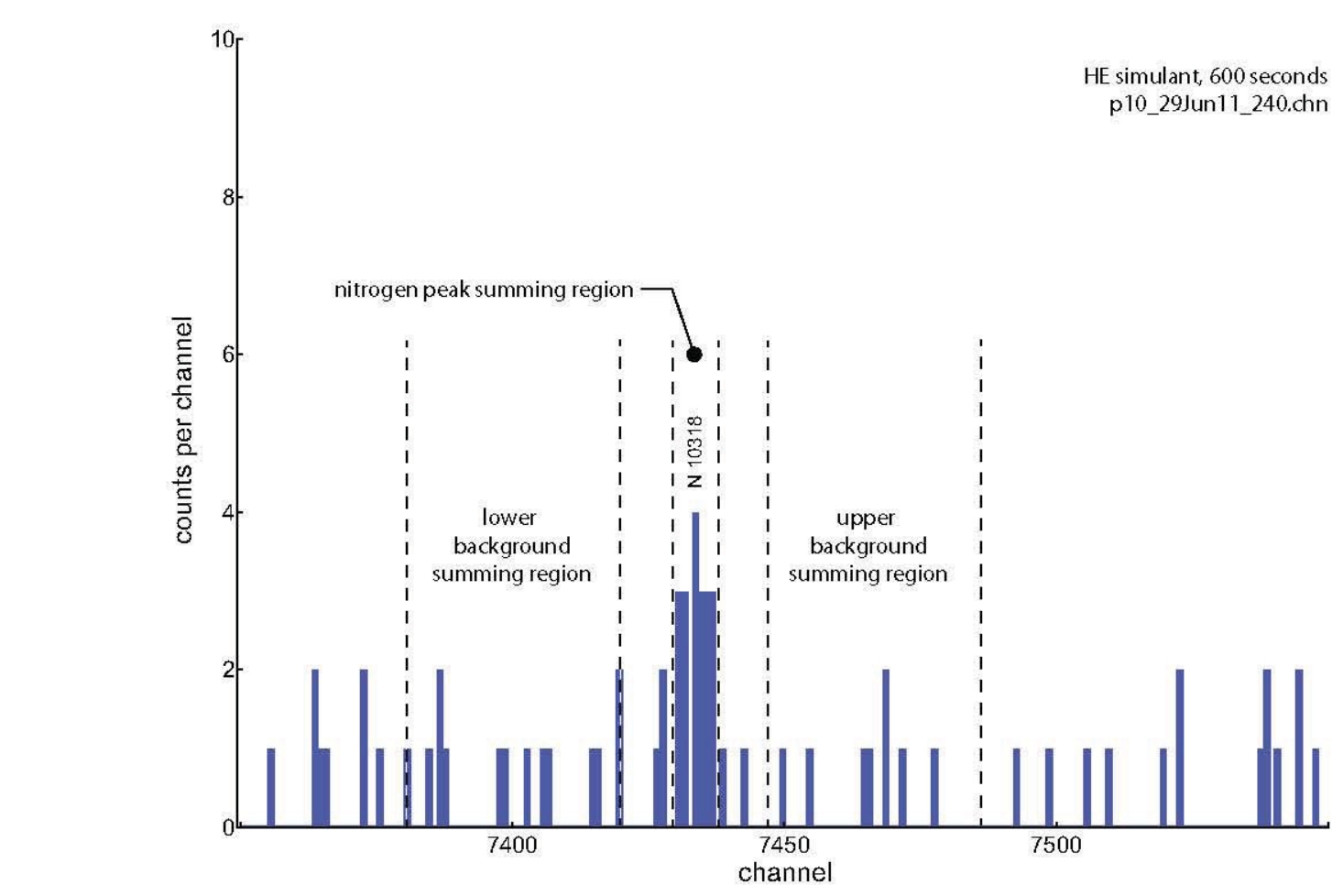

- When nitrogen is present in the sample, there are additional counts above background in the $10318 \mathrm{keV}$ and $10829 \mathrm{keV}$ regions.

The presence or absence of nitrogen in the unknown fill is ascertained by the degree to which the actual distribution of counts in the peak and background regions differs from a uniform distribution. This is determined (for the $10318 \mathrm{keV}$ and $10829 \mathrm{keV}$ peak regions separately) in the following way.

Consider that $k$ counts are obtained in the 9-channel peak region, while a total of $n$ counts are obtained for the two 40-channel background regions. Because the background in these regions is uniform, the number of background counts $\mathrm{B}$ in the peak region is estimated to be $\mathrm{B}=(n / 80) \times 9$. Thus the number of net counts $A$ in the peak region due to nitrogen gamma-rays is $A=k-B$. The metric $t^{*}=A / k_{1 / 2}$ can be interpreted as the net nitrogen peak area divided by the "peak uncertainty". Note that the value $t^{*}=0$ occurs when $k$ $=B$ (clearly no nitrogen) and when $k \leq B$ (any negative value of $t^{*}$ is set to zero), while the value $t^{*}=1$ is "suggestive" of the presence of nitrogen. This latter statement is borne out by our examinations of spectra from known HE fills.

Due to the typically small number of counts in the nitrogen peak regions, it is important to account for the background due to the liquid nitrogen used to cool the germanium detector. We experimentally determined this contribution to be approximately 1.15 counts/1000 livetime seconds for

Figure 9-2. Summing regions for the nitrogen single-escape peak. 
the $10318 \mathrm{keV}$ peak, and approximately 0.95 counts $/ 1000$ live seconds for the $10829 \mathrm{keV}$ peak. The fractional count $b$ (linear in livetime) due to this nitrogen background is accommodated by a slightly altered metric

$t^{*}=(k-B-b) /(k+b)^{1 / 2}$.

An extended comment on our formulation of $t^{*}$ : The accumulation of counts in the 9-channel peak region is a Poisson [3] process, since counts occur continuously and independently of one another. Thus, if the interrogation of a fill for a set time period was repeated many times, a Poisson distribution (for that set time period) would be created, with a mean count $\mathrm{k}$ and variance $\mathrm{\kappa}$ for the 9-channel peak region. Since we typically interrogate a fill only once, we assume $k \approx \kappa$, so $k^{1 / 2}$ is the standard deviation (or uncertainty, in units of counts) associated with the number of counts $k$. Thus $t^{*}$ is the ratio of the number of possible nitrogen counts (due to the fill) in the peak region, to the number of "uncertain" counts in the peak region. This ratio should be greater than one in order to believe that nitrogen has been detected.

Figure 9-3 shows the portion of the spectrum (for a nitrogencontaining simulant fill interrogated for 600 seconds) near the $10318 \mathrm{keV}$ single escape peak, with the 9-channel peak region and the two 40 -channel background summing regions indicated. In this case the peak contains $k=19$ gross counts. Since the two background regions contain $n=14+8=22$ counts, the background in the peak summing region $B=$ $(22 / 80) \times 9=2.47$ counts. The liquid nitrogen background $b=1.15 \mathrm{E}-3$ counts $/ \mathrm{sec}^{*} 600$ seconds $=0.69$ counts. Thus the net peak area $\mathrm{A}=19-2.47-0.69=15.84$ counts and the ratio $t^{*}{ }_{10318}=15.84 / \mathrm{sqrt}(19+0.69)=3.57$. A similar calculation for the nitrogen $10829-\mathrm{keV}$ full energy peak finds $t^{*}{ }_{10829}=1.48$, and adding $t^{*}$ values for both peaks, $t^{*}{ }_{10318}+t^{*}{ }_{10829}=t_{\text {sum }}^{*}=5.05$.

\section{Nitrogen Identification}

The measure of confidence that the unknown fill contains nitrogen is given by the value of the "signature function"

$$
\begin{aligned}
P_{N} & =0, \text { if } t_{\text {sum }}^{*}<1 \text {, where } t_{\text {sum }}^{*}=t^{*}{ }_{10318}+t^{*}{ }_{10829} \\
& =f \text {, if } 1 \leq t^{*}{ }_{\text {sum }} \leq 2 \\
& =1, \text { if } t_{\text {sum }}^{*}>2, \text { and } \\
f & =0.5-0.5 \cos \left[\pi\left(t_{\text {sum }}^{*}-1\right) /(2-1)\right] .
\end{aligned}
$$

Values $\mathrm{P}_{\mathrm{N}}=1$ and 0 indicate that the fill surely does, and surely does not, contain nitrogen; intermediate values indicate more or less confidence that the fill contains nitrogen.
The decision to use the sum $t^{*}{ }_{10318}+t^{*}{ }_{10829}$ is purely empirical: we find that the individual values $t^{*}{ }_{10318}$ and $t^{*}{ }_{10829}$ calculated for a known HE spectrum are rarely both large, and often one of the two is quite small. Similarly, the critical sum values 1 and 2 in the signature function above, while having a logical basis, were arrived at by interrogation of HE fills and simulants in many different types of containers and projectiles ranging from $75-\mathrm{mm}$ to $155-\mathrm{mm}$.

Figure 9-3 below displays the evolution of the nitrogen region of an explosive simulant. As more nitrogen counts accumulate in the peak regions during the interrogation, the corresponding $t_{\text {sum }}^{*}$ values increase.

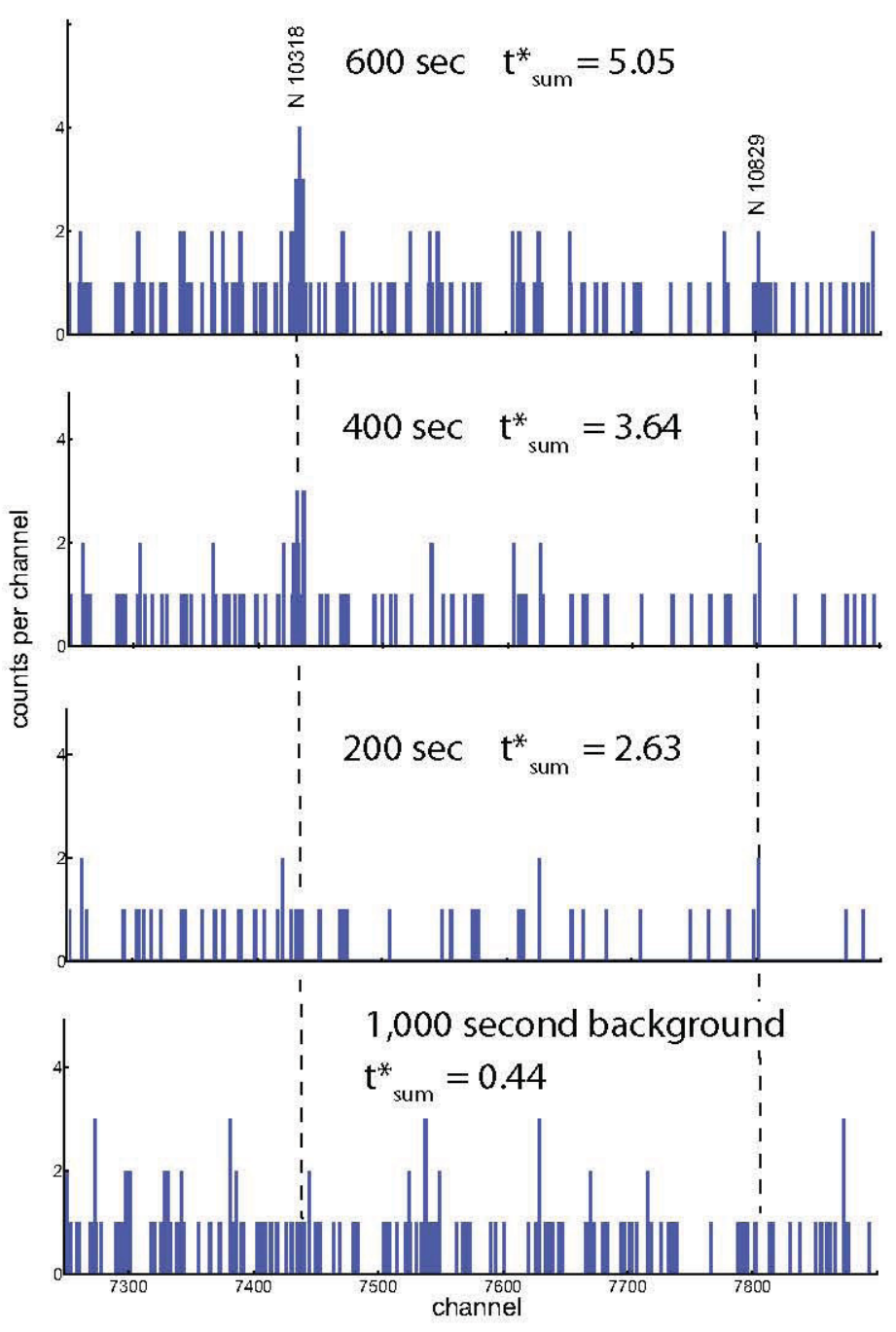

Figure 9-3. PINS spectrum nitrogen region for a background spectrum, and explosive simulant chemical spectra measured for 200,400, and 600 seconds. For the background spectrum, the $t^{*}{ }_{\text {sum }}=0.44$, and hence the nitrogen detection confidence level $P_{N}$ is 0 . For the other three spectra, $t^{*}{ }_{\text {sum }}>2$ in all cases, and the detection confidence level $P_{N}=1.0$. 
In Figure 9-4 below, the signature function $\mathrm{P}_{\mathrm{N}}$ is compared with the Poisson cumulative probability distribution for 2.4 background counts. The two functions, while they represent different approaches to assessing the likelihood that the fill contains nitrogen, produce very similar curves.

\section{Explosive Identification}

For explosive identification, the PINS software requires detection of hydrogen with $t^{*}=3.0$ or greater, and detection of nitrogen, as indicated by the signature values $t^{*}{ }_{10318}$ and $t^{*}{ }_{10829}$ summing to 1.8 or greater and producing a confidence level $\mathrm{P}_{\mathrm{N}} \geq 0.9$.

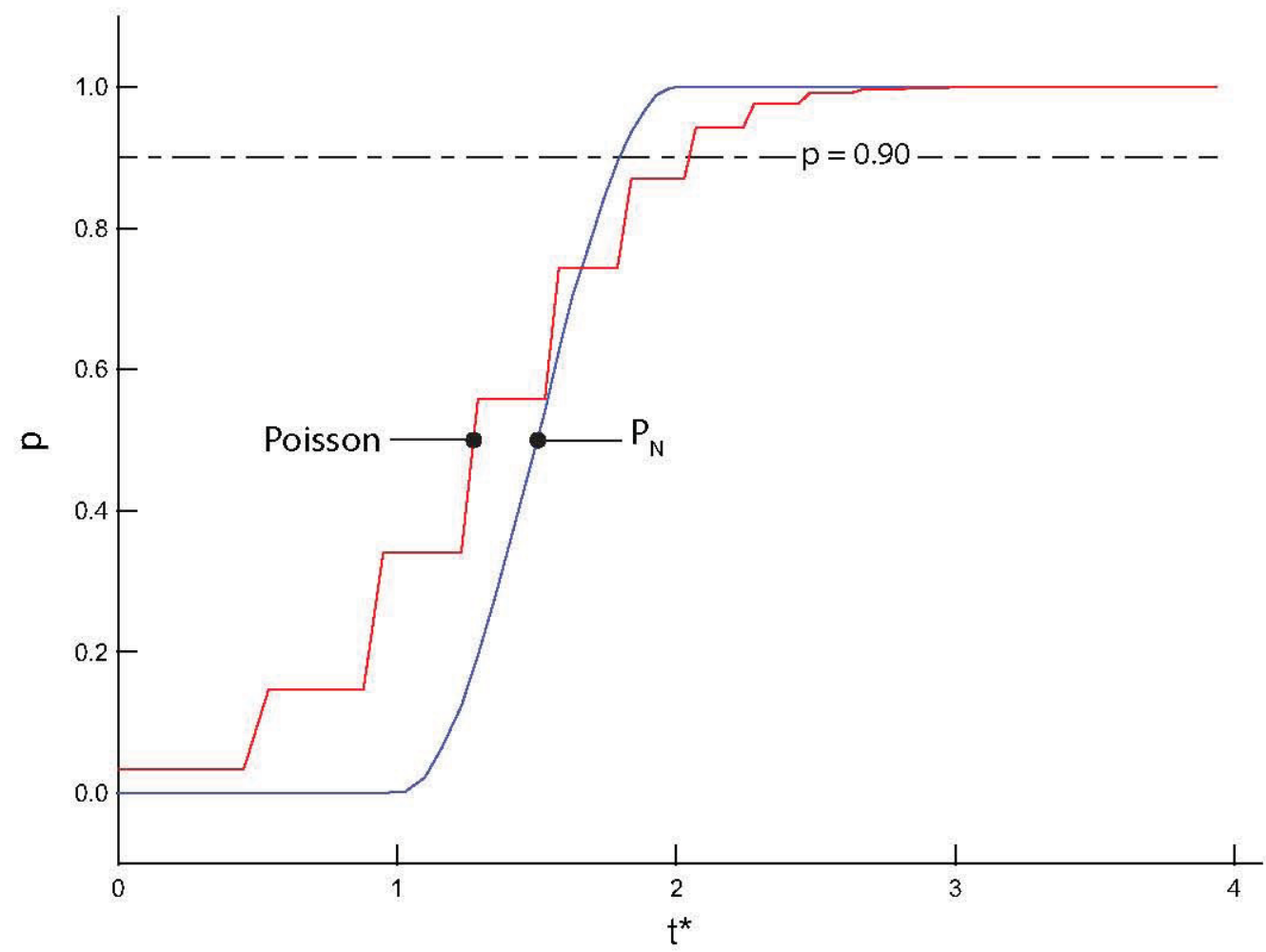

Figure 9-4. Comparison of the Poisson cumulative distribution function with the PINS $P_{N}$ function.

\section{References}

1. J. Köhler and R. Meyer, Explosives, Fourth Edition (New York: VCH Publishers, 1993) page 63, 70-73, and 371-373.

2. M.J. O'Neil, ed. The Merck Index, 13th Edition (Whitehouse Station, NJ: Merck \& Co., Inc., 2001) article 5157. This article discusses the isomer, isobutylene. The rubber-like polymer polyisobutylene is a long chain of the $\mathrm{C}_{4} \mathrm{H}_{8}$ isomers.
3. Poisson statistics are described in the context of radiation counting in G.F. Knoll, Radiation Detection and Measurement, Third Edition (New York: John Wiley \& Sons, 2000) pages 73-75. An introductorylevel discussion of Poisson statistics that also includes radiation-counting examples can be found in J.R. Taylor, An Introduction to Error Analysis: The Study of Uncertainties in Physical Measurements, Second Edition (Sausalito, CA: University Science Books, 1997. A more advanced discussion can be found in S.M. Ross, Introduction to Probability and Statistics for Engineers and Scientists, Second Edition (San Diego, CA: Academic Press, 2000) pages 144-150. 


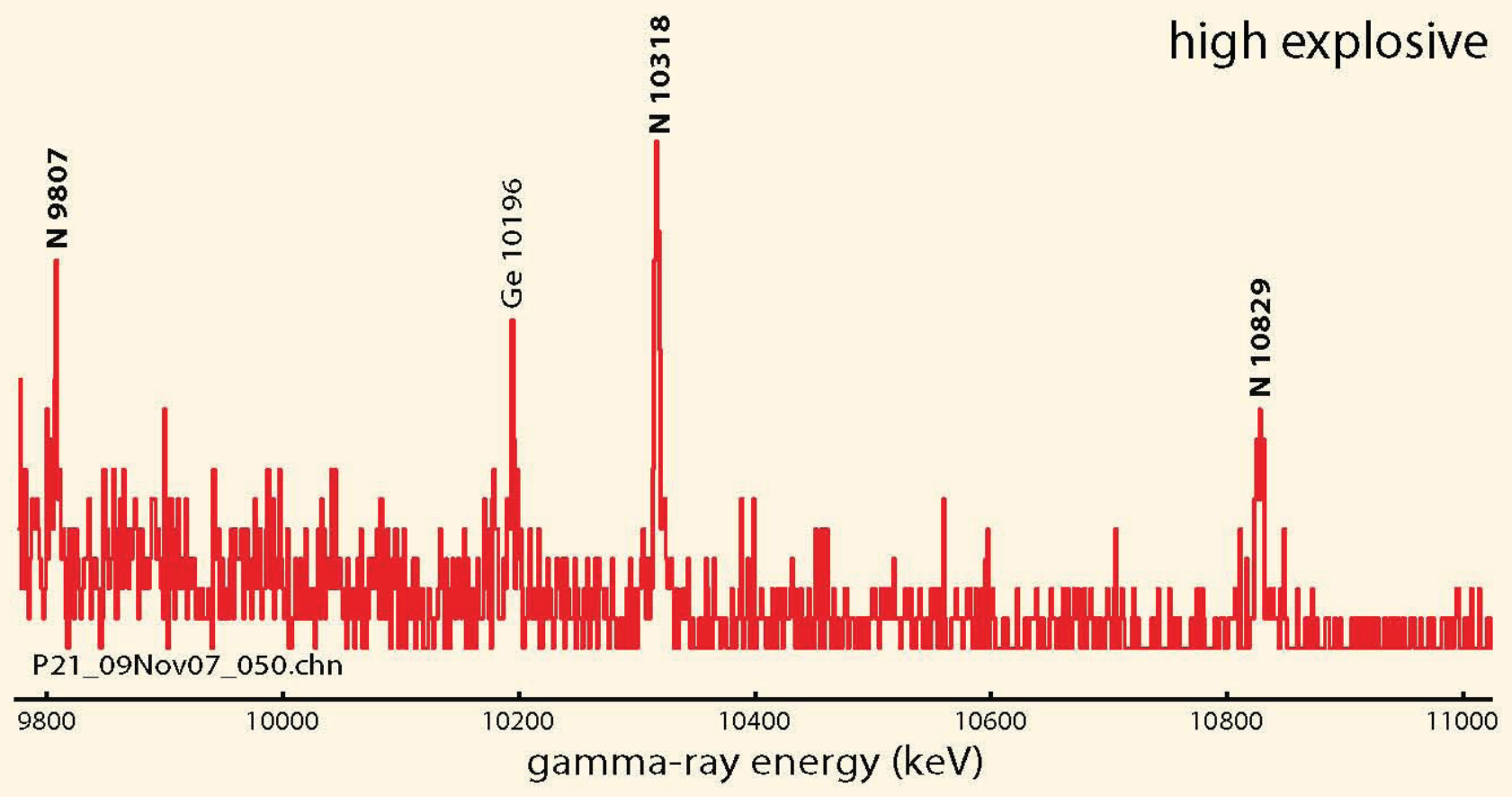

\section{Key spectral features}

- Weak nitrogen peak(s) at 10318 and/or $10829 \mathrm{keV}$

- Moderate or strong hydrogen peak at $2223 \mathrm{keV}$

\section{Spectrum ID Tips}

- It may take 1,000 to 3,000 seconds for a detectable nitrogen peak to appear in the PINS spectrum of an explosive.

- Hydrogen will be evident in 100 seconds.

- Because the nitrogen concentration varies among explosives, the identification time varies too.

- The nitrogen single-escape peak at 10318 is usually the strongest of the nitrogen peaks.

\section{Caveats}

- The nitrogen double-escape peak at $9807 \mathrm{keV}$ is only observed from large explosive objects, like the Composition B-filled $155-\mathrm{mm}$ artillery shell that produced the spectrum above.

- Smaller explosive objects, e.g. 75-mm artillery shells, may take 3,000 seconds to identify.

- PINS is not sufficiently sensitive to nitrogen to reliably identify the explosive burster in chemical and smoke munitions.

- The spectrum was measured from a $155-\mathrm{mm}$ artillery projectile.
Explosive chemical example: trinitrotoluene

Chemical formula: $\mathrm{C}_{7} \mathrm{H}_{5} \mathrm{~N}_{3} \mathrm{O}_{6}$

Key elements: hydrogen 2.2 wt- $\%$, nitrogen 18.5 wt- $\%$ NATO Symbol: HE
Chemical type: explosive

CWC schedule: $n / a$

AKA: TNT 
82 PINS Spectrum Identification Guide 


\section{Chapter 10}

Practice Munition Fills: Boron,

Calcium, Silicon, and Sodium-Based Chemicals

\section{Practice Fills}

For economy and safety, military practice munitions are often filled with inert substances. Concrete, plaster-ofParis, and sand are frequent practice fills. For chemical warfare munition practice fills, a water/antifreeze mixture is common, and decontamination bleach is sometimes used.

\section{Spectrum Identification}

The practice fill chemicals are based on boron, calcium, silicon, and sodium, and the practice-fill identification logic is based on the same chemical elements, as shown in Figure 10-1.

Boron and hydrogen are the key elements for identification of water/antifreeze (W/AF) mixtures. Modern automotive antifreeze is mainly ethylene glycol, and it is usually mixed with an equal amount of water. Under neutron bombardment, both ethylene glycol and water produce strong hydrogen gamma rays. Boron, in the form of sodium tetraborate decahydrate, is added to antifreeze as a corrosion inhibitor.[1] Even a small concentration of boron produces a large gamma ray peak at $477 \mathrm{keV}$, because boron-10 has a remarkably large thermal neutron capture cross section, 3837 barns.[2]

Calcium can indicate three practice fills. If sulfur and hydrogen are also present, the practice fill is plaster-ofParis (POP), calcium sulfate dihydrate.[3] Alternatively, if chlorine is present, the fill is calcium-hypochlorite bleach, a type of decon bleach.[4] Calcium is also found in concrete.

Sodium hypochlorite is another type of decon bleach. [5] Chlorox bleach is a commercial example.

Silicon alone indicates a sand practice fill. Sand is largely silicon dioxide. [6] Silicon in combination with calcium and hydrogen identifies a concrete practice fill.[7]

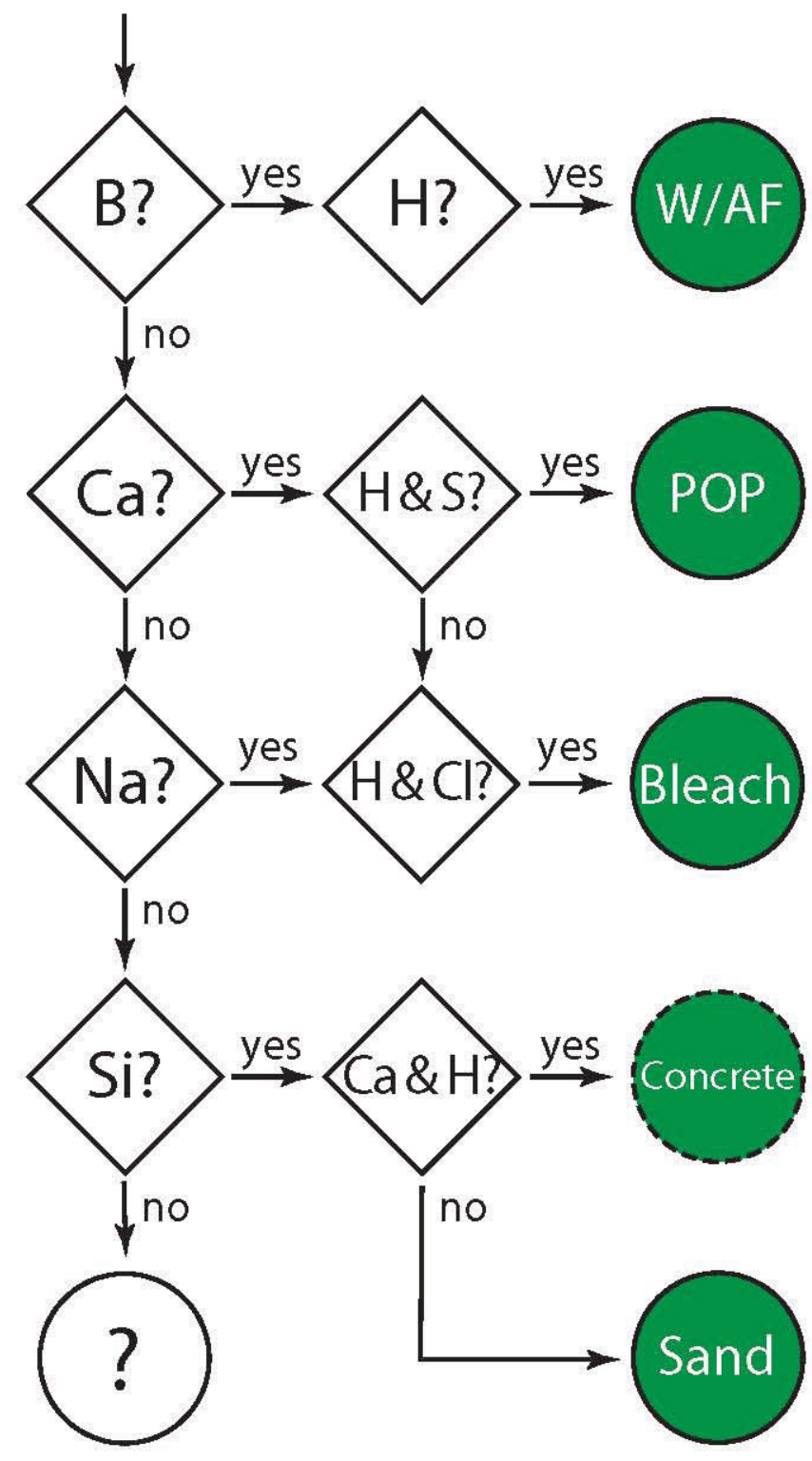

Figure 10-1: PINS decision tree branch for practice-fill chemicals. 


\section{References}

1. The current military specification for antifreeze, MILA- $46153 \mathrm{C}$, requires it to contain 4 weight- $\%$ sodium tetraborate decahydrate.

Federal and military specifications have required boron additives to antifreeze since 1956. See C.B. Jordan, "U.S. Army Antifreeze after 50 Years," Engine Coolant Testing: Second Symposium, ASTM STP 887, R. E. Beal, Ed., American Society for Testing and Materials, Philadelphia, 1986, pages 249-255.

2. S.F. Mughabghab, M. Divadeenam, and N.E. Holden, Neutron Cross Sections, Vol. 1 (New York: Academic Press, 1981) page 5-1.

3. M.J. O'Neil, Editor, The Merck Index, Thirteenth Edition (Whitehouse Station, New Jersey: Merck \& Co., Inc., 2001) article 1711.

4. The Merck Index, article 1676.

5. The Merck Index, article 8702 .

6. P.W. Atkins, General Chemistry (New York: W.H. Freeman and Co., 1989) page 703. The Merck Index, article 8567.

7. J.E msley, Nature's Building Blocks: An A-Z Guide to the Elements (Oxford, United Kingdom: Oxford University Press, 2001) page 87. 


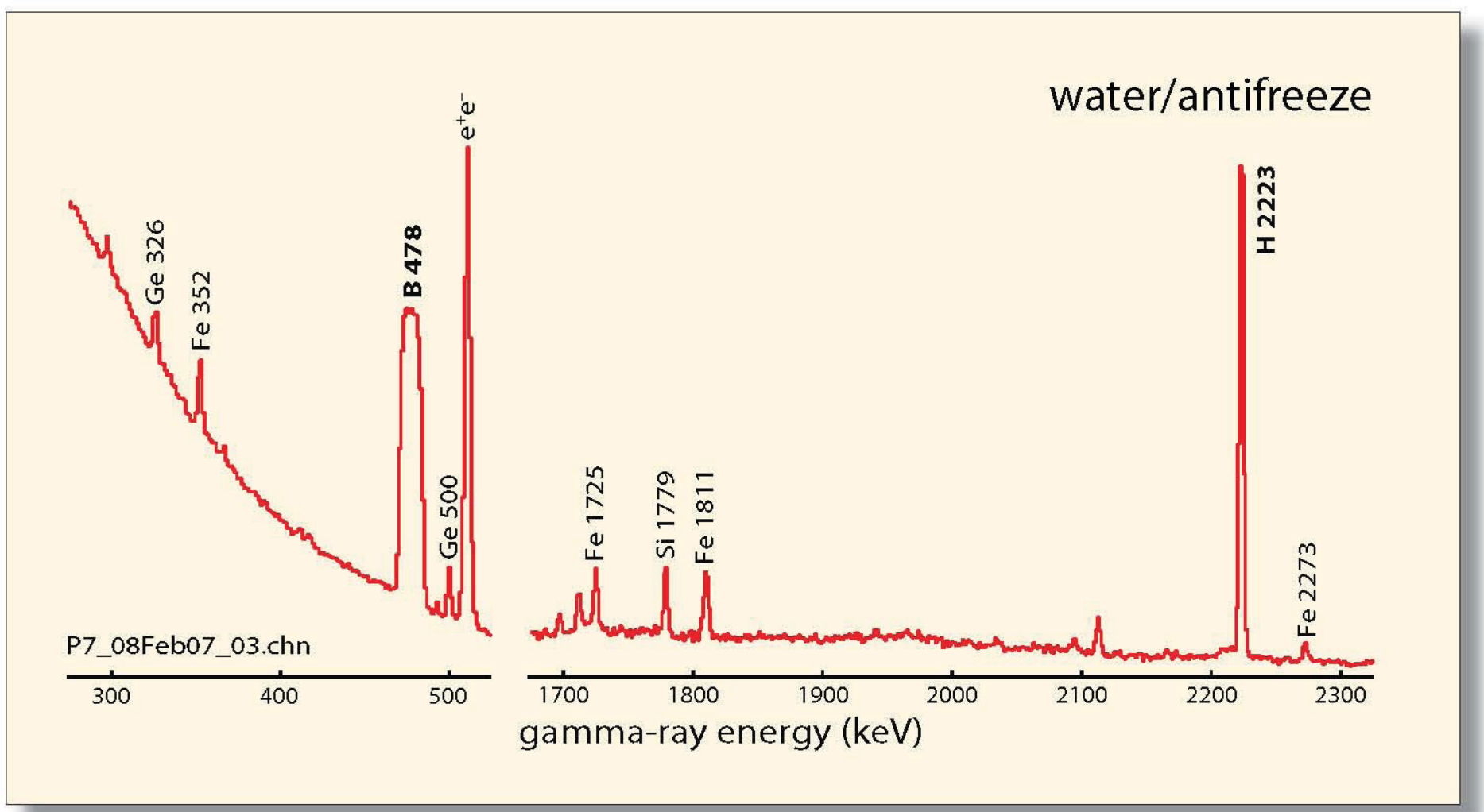

\section{Key spectral features}

- Strong or very strong boron peak at $478 \mathrm{keV}$

- Strong or very strong hydrogen peak at $2223 \mathrm{keV}$

\section{Spectrum ID Tips}

- The boron peak is Doppler-broadened.

\section{Caveats}

- Boron in the neutron shielding of the PINS Cf-252 source shipping bucket can induce a false positive for boron in spectra from the item under test.

- Keep the shipping bucket at least 10 feet away from the PINS system during assays.

- The spectrum was measured from a 4.2-inch mortar projectile replica.
Chemical names: water, ethylene glycol, sodium tetraborate decahydrate

Chemical mixture: $\mathrm{Na}_{2} \mathrm{~B}_{4} \mathrm{O}_{7} \cdot \mathrm{H}_{2} \mathrm{O}$

Key elements: boron $0.7 w t-\%$, hydrogen $10.3 w t-\%$, sodium $0.2 w t-\%$

NATO Symbol: $n / a$
Chemical type: practice fill

CWC schedule: $n / a$ 
86 PINS Spectrum Identification Guide 


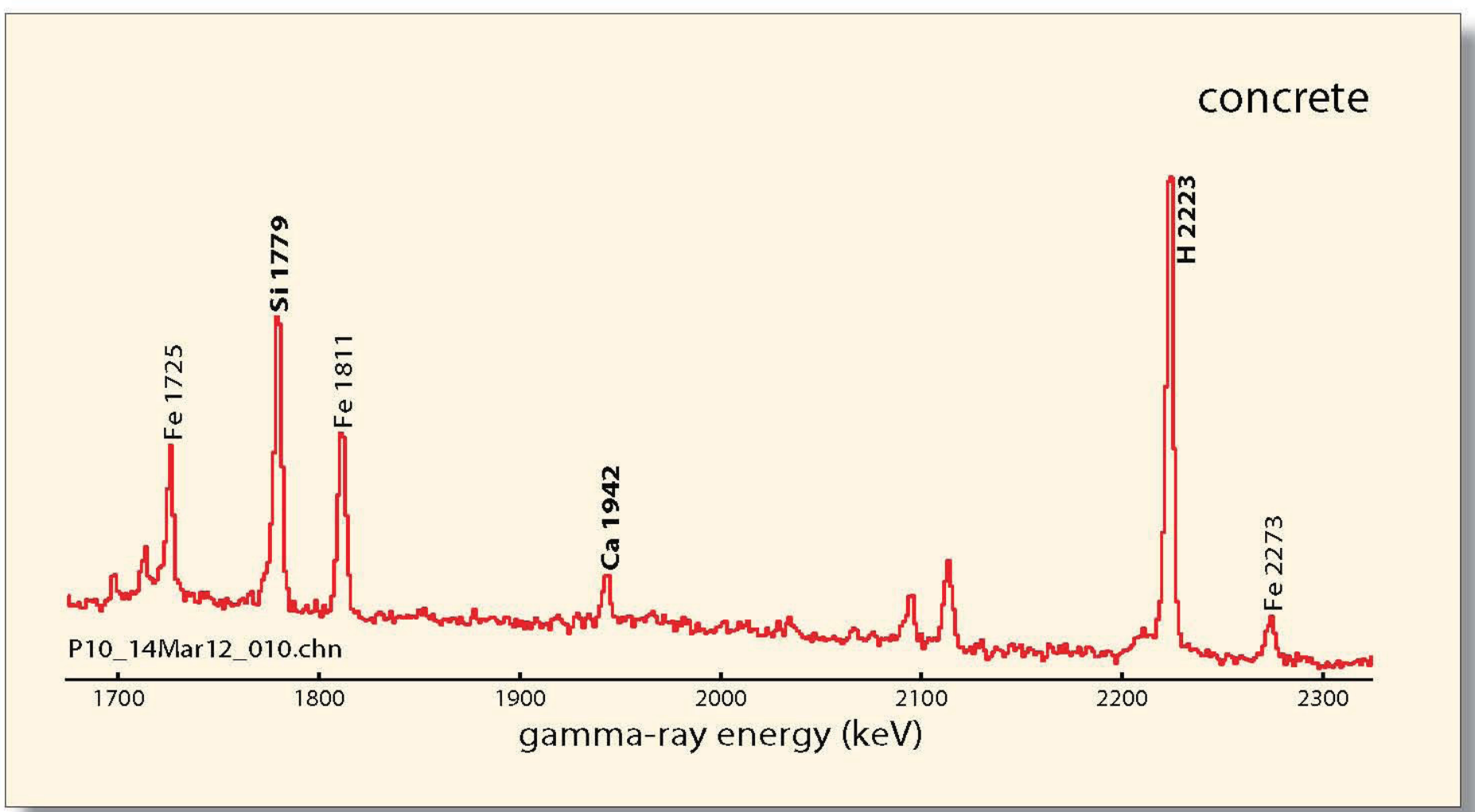

\section{Key spectral features}

- Moderate or strong calcium peak at $1942 \mathrm{keV}$

- Strong silicon peak at $1779 \mathrm{keV}$

- Moderate or strong hydrogen peak at $2223 \mathrm{keV}$

\section{Spectrum ID Tips}

- The 1779-keV silicon peak will be stronger than the calcium or hydrogen peak.

\section{Caveats}

- Calcium, hydrogen, and silicon peaks commonly appear as background peaks in PINS spectra. Be sure to measure and subtract background before concluding a munition is filled with concrete.

- The spectrum was measured from a 4.2-inch mortar projectile.

- PINS spectra have been measured from less than a dozen concrete-filled munitions.
Chemical name: concrete

Chemical formula: mixture of Portland cement, crushed rock, sand, and water.

Key elements: calcium, hydrogen, silicon

NATO Symbol: $n / a$
Chemical type: practice fill

CWC schedule: $n / a$

AKA: Portland concrete 

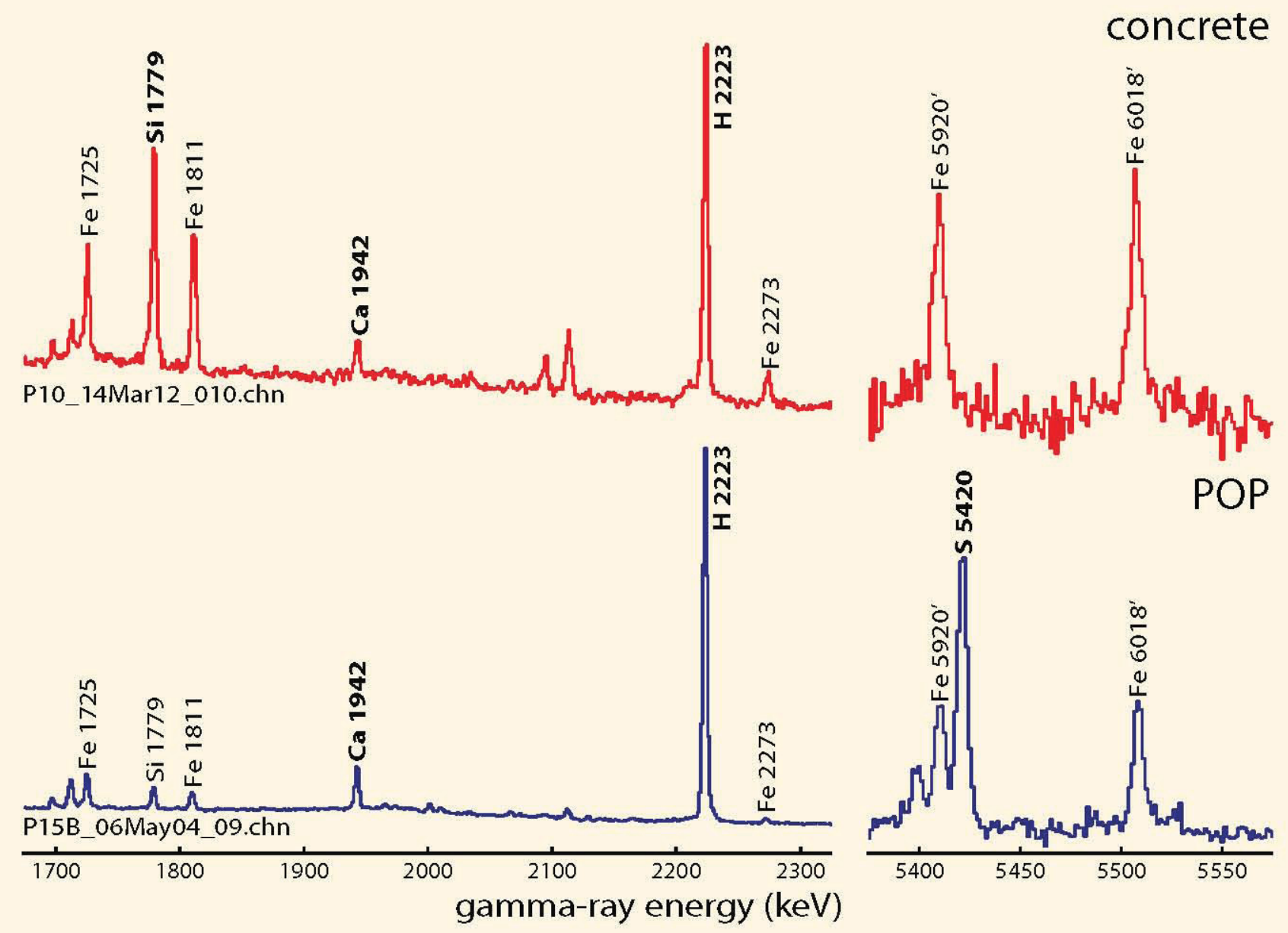

\section{Spectral comparison}

The figure above compares PINS spectra of concrete (red curve) and plaster-of-Paris (blue). The concrete PINS spectrum was measured from a 4.2-inch mortar projectile, and the plaster-of-Paris (POP) spectrum was measured from a 1,000 pound aerial bomb.

\section{Distinguishing features}

- POP contains sulfur, but not silicon.

- Concrete contains silicon, but not sulfur.
Chemical name: concrete

Chemical formula: mixture of Portland cement, crushed rock, sand, and water.

Key elements: calcium, hydrogen, silicon

NATO Symbol: $n / a$
Chemical type: practice fill

CWC schedule: $n / a$

AKA: Portland concrete 


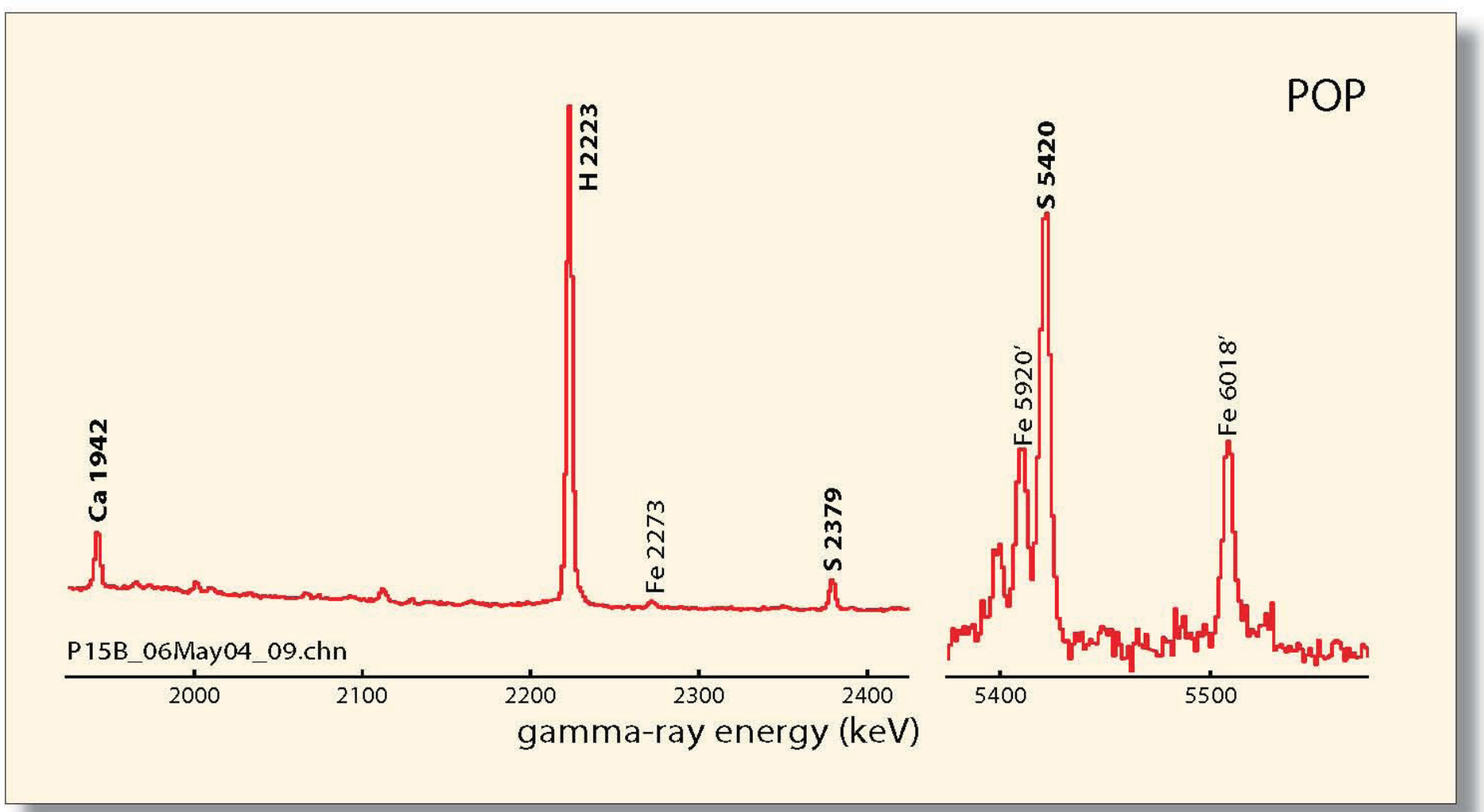

\section{Key spectral features}

- Moderate or strong calcium peak at $1942 \mathrm{keV}$

- Strong hydrogen peak at $2223 \mathrm{keV}$

- Moderate sulfur peak at $5420 \mathrm{keV}$

\section{Spectrum ID Tips}

- A weak or moderate sulfur peak may also be observed at $2379 \mathrm{keV}$.

- It's often easier to see the calcium, hydrogen, and 2379-keV sulfur peak if the spectrum is viewed with a logarithmic vertical scale.

- The 2223-keV hydrogen peak will be larger than the 1942-keV calcium peak or the 2379-keV sulfur peak.

\section{Caveats}

- Calcium and hydrogen peaks commonly appear as background peaks in PINS spectra. Be sure to measure and subtract background before concluding a munition is filled with plaster-of-Paris.

- EOD soldiers sometimes apply a plaster-of-Paris (POP) bandage to a recovered munition as a render-safe measure. PINS is not sensitive to the small amount of POP in these bandages.

- The spectrum was measured from a 1,000-pound Navy aerial practice bomb.

- PINS spectra have been measured from less than twenty POP-filled munitions.
Chemical name: calcium sulfate dihydrate

Chemical formula: $\mathrm{CaSO}_{4} \cdot 2 \mathrm{H}_{2} \mathrm{O}$

Key elements: calcium 32.3 wt- $\%$, hydrogen 3.2 wt- $\%$, sulfur 25.8 wt-\%

\section{Chemical type: practice fill}

CWC schedule: $n / a$

AKA: plaster-of-Paris, POP

NATO Symbol: $n / a$ 


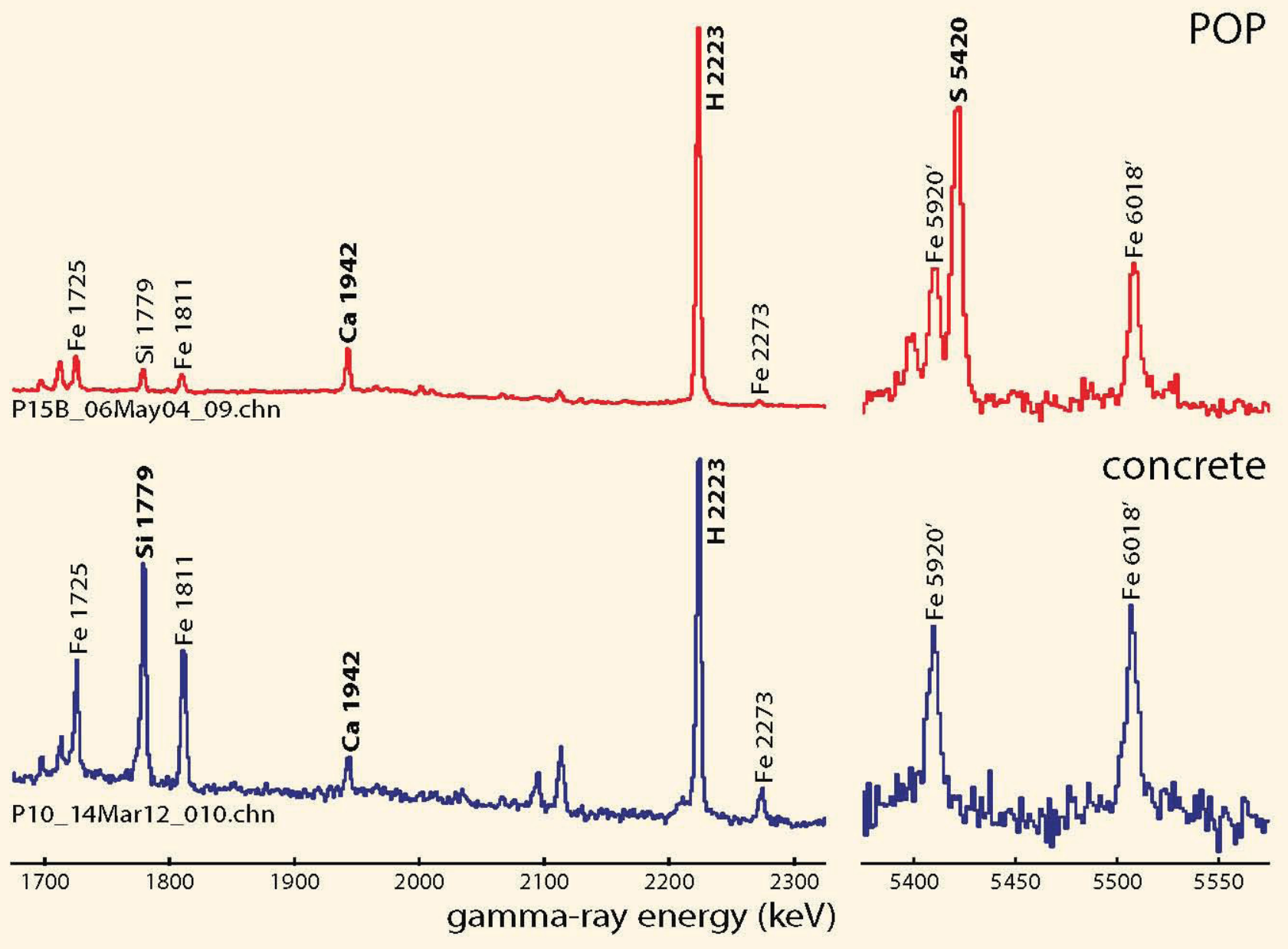

\section{Spectral comparison}

The figure above compares PINS spectra of concrete (blue curve) and plaster-of-Paris (red). The concrete PINS spectrum was measured from a 4.2-inch mortar projectile, and the plaster-of-Paris (POP) spectrum was measured from a 1,000 pound aerial bomb.

\section{Distinguishing features}

- POP contains sulfur, but not silicon.

- Concrete contains silicon, but not sulfur.
Chemical name: calcium sulfate dihydrate

Chemical formula: $\mathrm{CaSO}_{4} \cdot 2 \mathrm{H}_{2} \mathrm{O}$

Key elements: calcium 32.3 wt- $\%$, hydrogen 3.2 wt- $\%$, sulfur 25.8 wt- $\%$
Chemical type: practice fill

CWC schedule: $n / a$

AKA: plaster-of-Paris, POP

NATO Symbol: $n / a$ 


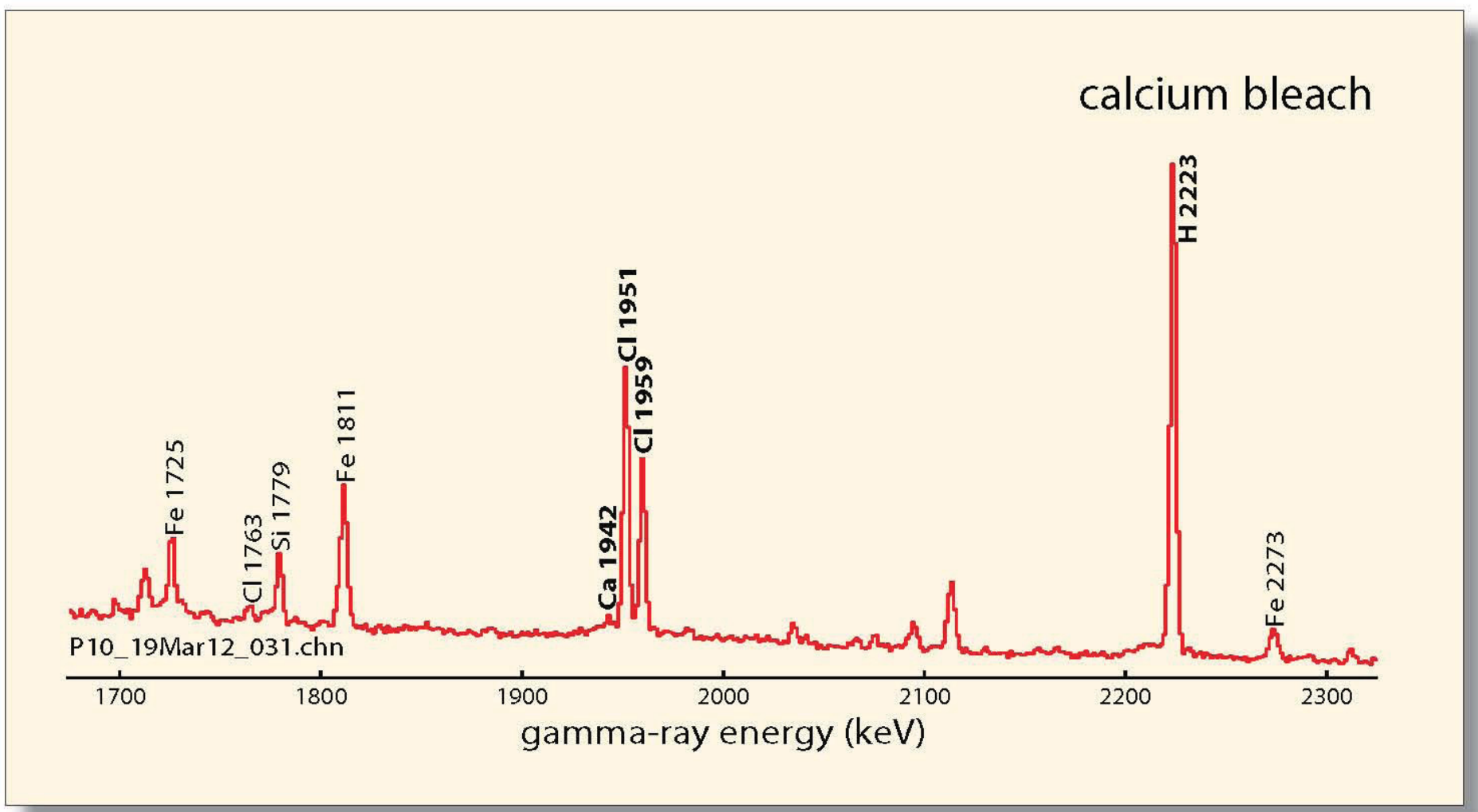

\section{Key spectral features}

- Weak or moderate calcium peak at $1942 \mathrm{keV}$

- Moderate or strong chlorine peaks at 1165, 1951, and $1959 \mathrm{keV}$

- Strong or very strong hydrogen peak at $2223 \mathrm{keV}$

\section{Spectrum ID Tips}

- The chlorine-to-hydrogen ratio, (Cl 1959/H 2223), is less than 0.5 .

\section{Caveats}

- The chlorine-to-hydrogen ratio for decon bleach is variable, depending on dilution, but always less than 0.5 .

- If the chlorine-to-hydrogen ratio > 1.0, the fill chemical is likely a chemical warfare agent.

- "Super tropical bleach" (STB) is another type of calciumbased decon bleach.

- The spectrum was measured from a 4.2-inch mortar projectile replica.

- PINS spectra have been measured from less than a handful of munitions filled with calcium decon bleach.
Chemical name: calcium hypochlorite dissolved in water

Chemical mixture: $\mathrm{CaO}_{2} \mathrm{Cl}_{2} 5 \%, \mathrm{H}_{2} \mathrm{O} 95 \%$

Key elements: chlorine 2.5 wt- $\%$, hydrogen 10.6 wt- $\%$, calcium 1.4 wt- $\%$
Chemical type: practice fill

CWC schedule: $n / a$

AKA: HTH bleach

NATO Symbol: $n / a$ 

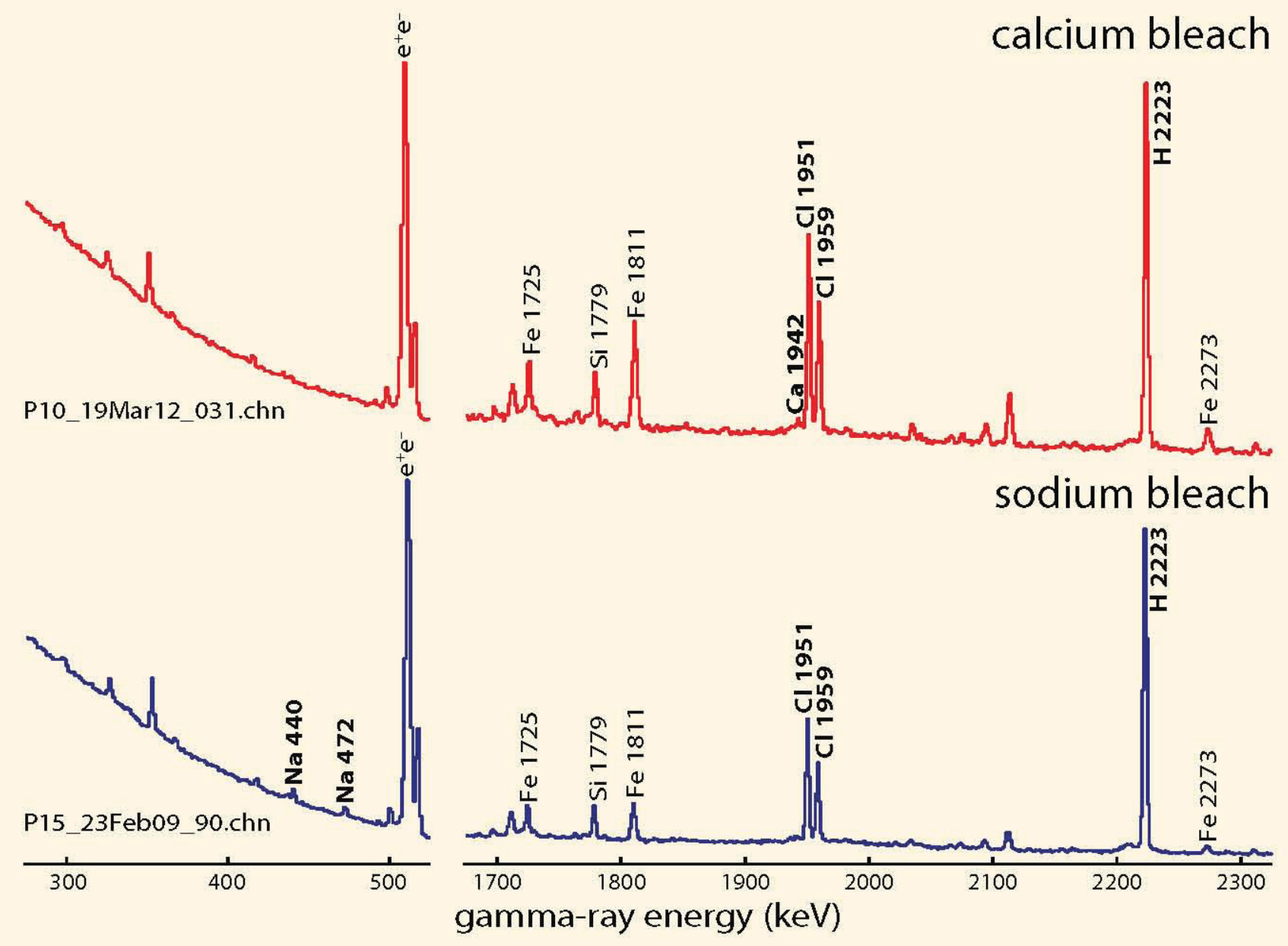

\section{Spectral comparison}

The figure above compares PINS spectra of calcium hypochlorite bleach (red curve) and sodium hypochlorite bleach (blue). Both spectra were measured from 4.2-inch mortar projectiles.
Distinguishing features

- Sodium bleach contains sodium, chlorine, and hydrogen.

- Calcium bleach contains calcium, chlorine, and hydrogen.

- A weak or moderate 440-keV sodium peak can often be observed in sodium bleach spectra.

- A weak 1942-keV calcium peak may be observed in calcium bleach spectra.
Chemical name: calcium hypochlorite dissolved in water Chemical mixture: $\mathrm{CaO}_{2} \mathrm{Cl}_{2} 5 \%, \mathrm{H}_{2} \mathrm{O} 95 \%$

Key elements: chlorine 2.5 wt- $\%$, hydrogen 10.6 wt- $\%$, calcium 1.4 wt- $\%$
Chemical type: practice fill

CWC schedule: $n / a$

AKA: HTH bleach

NATO Symbol: $n / a$ 


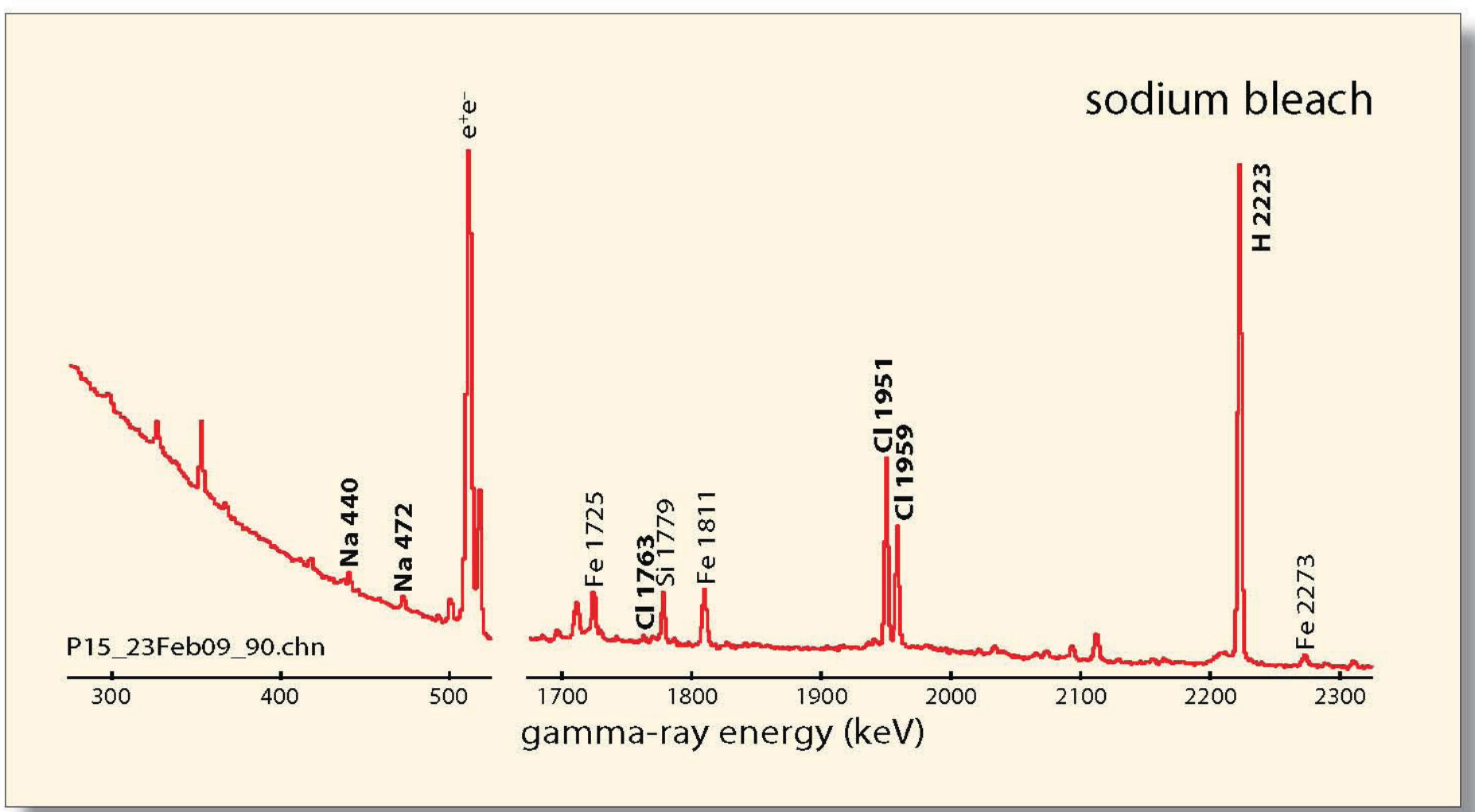

\section{Key spectral features}

- Weak or moderate sodium peak at $472 \mathrm{keV}$

- Moderate or strong chlorine peaks at 1165, 1951, and $1959 \mathrm{keV}$

- Strong or very strong hydrogen peak at $2223 \mathrm{keV}$

\section{Spectrum ID Tips}

- The chlorine-to-hydrogen ratio, (Cl 1959/H 2223), is less than 0.5 .

\section{Caveats}

- The chlorine-to-hydrogen ratio for sodium decon bleach is variable, depending on dilution, but always less than 0.5 .

- If the chlorine-to-hydrogen ratio $>1.0$, the fill is likely a chemical warfare agent.

- The spectrum was measured from a 4.2-inch mortar projectile replica.
Chemical name: sodium hypochlorite dissolved in water Chemical mixture: $\mathrm{NaOCl} 5 \%, \mathrm{H}_{2} \mathrm{O} 95 \%$

Key elements: chlorine 2.4 wt- $\%$, hydrogen 10.6 wt- $\%$, sodium 1.2 wt- $\%$

NATO Symbol: $n / a$
Chemical type: practice fill

CWC schedule: $n / a$

AKA: Chlorox, sodium decon bleach 


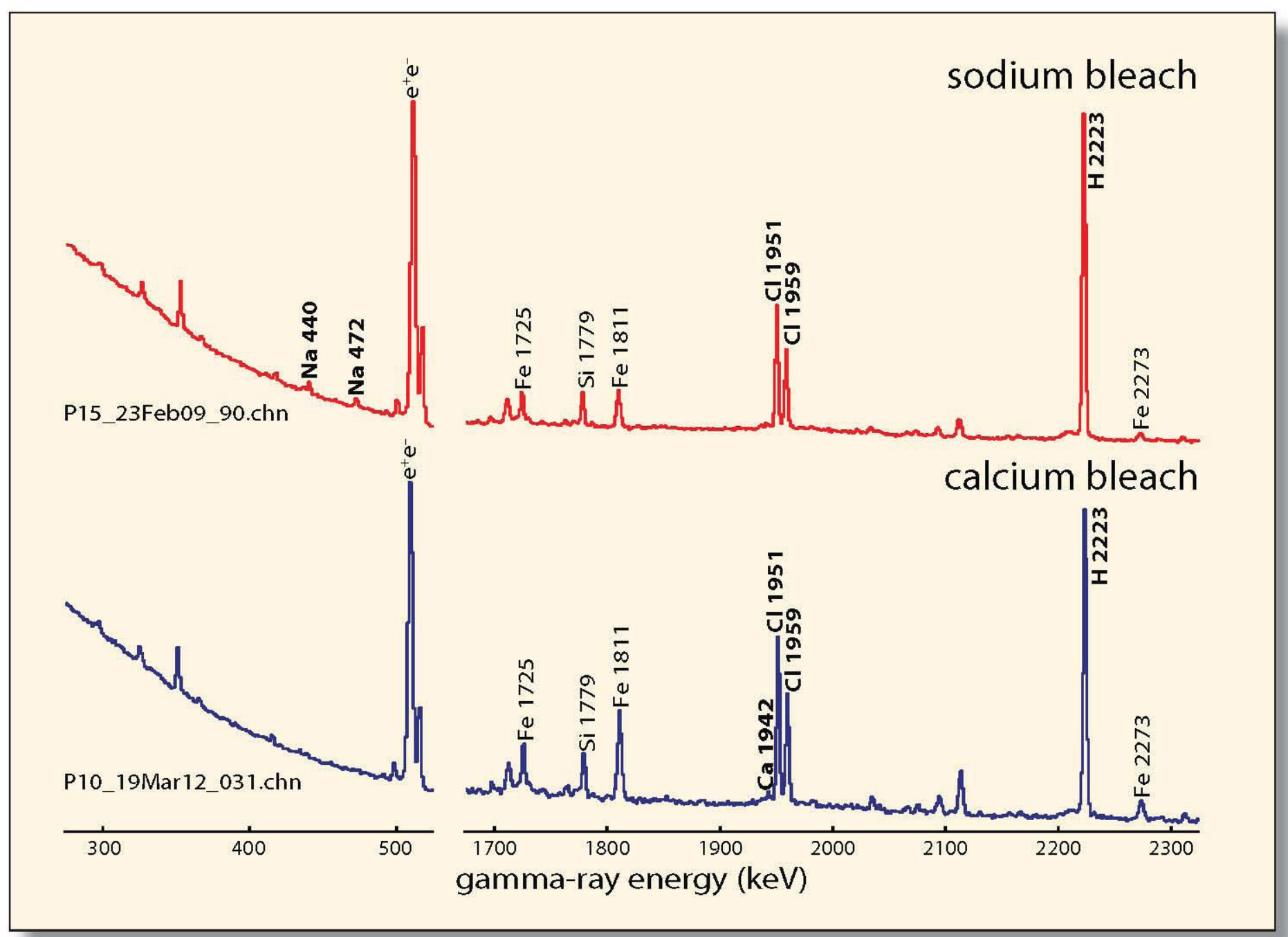

\section{Spectral comparison}

The figure above compares PINS spectra of calcium hypochlorite bleach (blue curve) and sodium hypochlorite bleach (red). Both spectra were measured from 4.2-inch mortar projectiles.
Distinguishing features

- Sodium bleach contains sodium, chlorine, and hydrogen.

- Calcium bleach contains calcium, chlorine, and hydrogen.

- A weak or moderate 440-keV sodium peak can often be observed in sodium bleach spectra.

- A weak 1942-keV calcium peak may be observed in calcium bleach spectra
Chemical name: sodium hypochlorite dissolved in water Chemical mixture: $\mathrm{NaOCl} 5 \%, \mathrm{H}_{2} \mathrm{O} 95 \%$

Key elements: chlorine 2.4 wt- $\%$, hydrogen 10.6 wt- $\%$, sodium 1.2 wt- $\%$

NATO Symbol: $n / a$
Chemical type: practice fill

CWC schedule: $n / a$

AKA: Chlorox, sodium decon bleach 


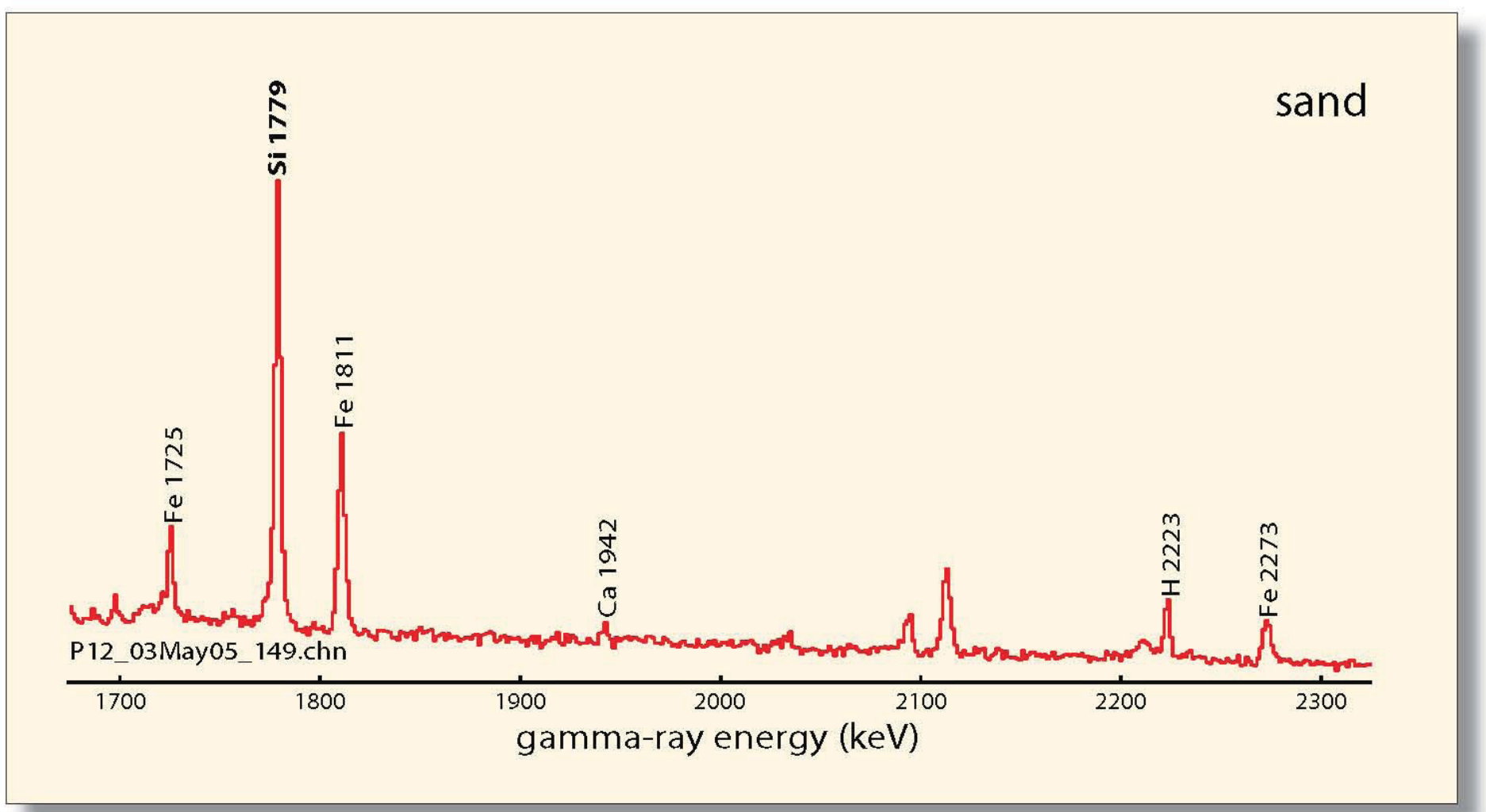

\section{Key spectral features}

- Strong or very strong silicon peak at $1779 \mathrm{keV}$

- Moderate silicon peak at $3539 \mathrm{keV}$

\section{Spectrum ID Tips}

- The sand silicon peak will be at least a factor of two larger than the 2223-keV hydrogen peak.

\section{Caveats}

- Silicon and hydrogen peaks commonly appear as background peaks in PINS spectra. Be sure to measure and subtract background before concluding a munition is filled with sand.

- The spectrum was measured from a 4.2-inch mortar projectile replica.
Chemical name: silicon dioxide

Chemical formula: $\mathrm{SiO}_{2}$

Key elements: silicon 46.7 wt- $\%$

NATO Symbol: $n / a$
Chemical type: practice fill

CWC schedule: $n / a$

AKA: silica 

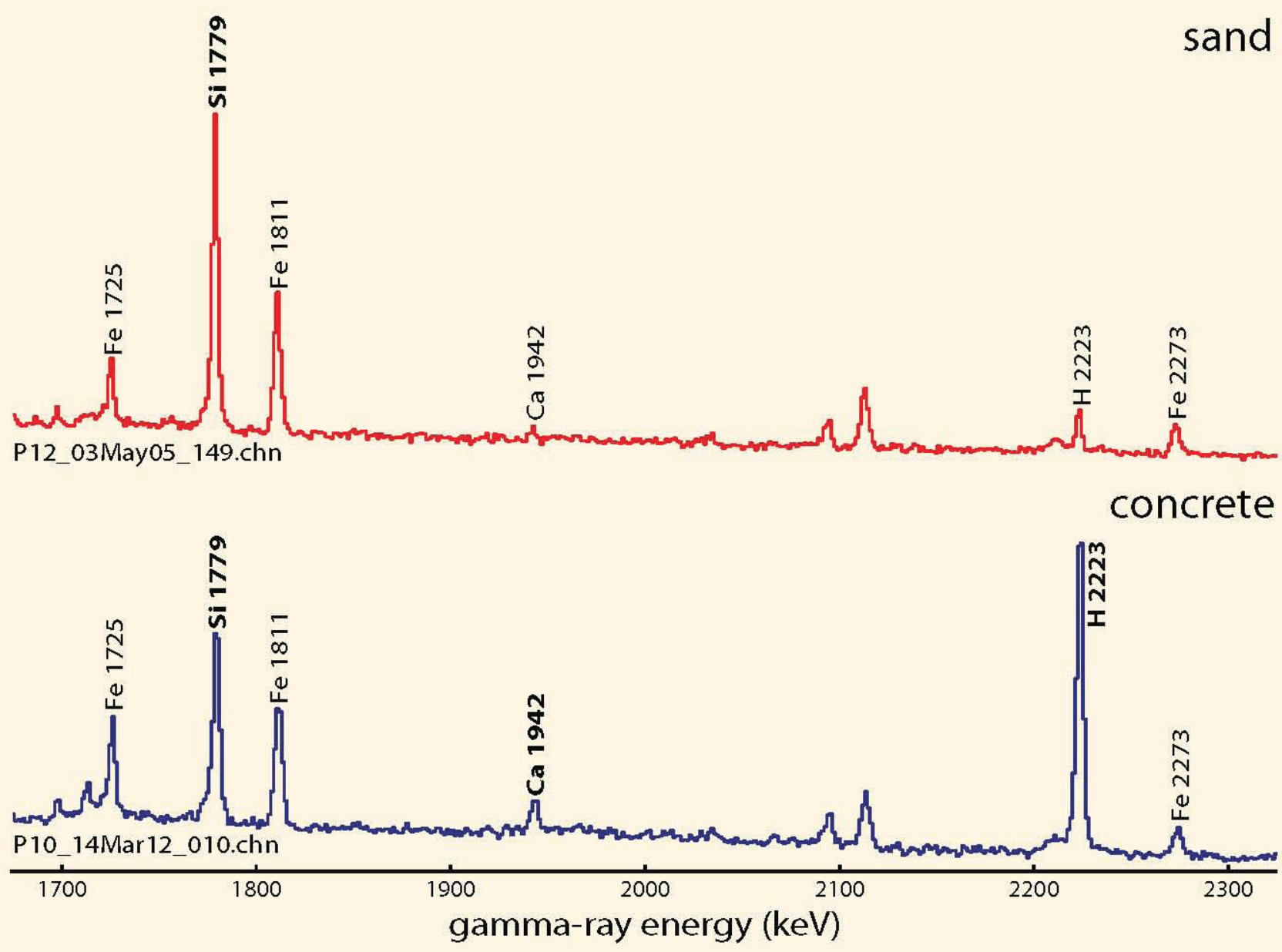

\section{Spectral comparison}

The figure above compares PINS spectra of sand (red curve) and concrete (blue). Both the concrete and the sand spectra were measured from 4.2-inch mortar projectiles.

\section{Distinguishing features}

- Sand contains only silicon and oxygen.

- Concrete contains calcium, silicon, hydrogen and oxygen.

- Concrete may contain other elements as well, for example aluminum, depending on the content of the crushed stone or aggregate added to the mix.
Chemical name: silicon dioxide

Chemical formula: $\mathrm{SiO}_{2}$

Key elements: silicon 46.7 wt-\%

NATO Symbol: $n / a$
Chemical type: practice fill

CWC schedule: $n / a$

AKA: silica 


\section{Appendix A}

\section{Hazardous Industrial Chemicals}

In addition to chemical warfare agents and explosives, industrial chemicals also can be identified nondestructively by PINS. For brevity, this chapter is limited to just four examples of hazardous industrial chemicals.

Three of the industrial chemicals discussed below are compressed gases, and one is a dense liquid. The gases would be found in compressed gas cylinders, of course, and the liquid in either a glass or metal container. PINS has been used to identify these and other industrial chemicals found in unlabeled containers. Figure A-1 presents the chemical identification logic limited to the four industrial chemicals, plus a related compressed gas.

\section{Acetylene Identification}

If silicon is detected from a compressed-gas cylinder along with calcium plus a strong or very strong hydrogen peak, the cylinder contains the welding gas acetylene, $\mathrm{C}_{2} \mathrm{H}_{2}$. [1]

Why the silicon and calcium? Acetylene cannot be safely stored by simply compressing it; it tends to explode at pressures above $15 \mathrm{psi}$. Acetylene can be safely stored in acetone-filled porous ceramic media. Each volume of acetone can hold 300 volumes of dissolved acetylene at 12 atmospheres.[2] The calcium and silicon gamma rays are produced in the ceramic material, and the hydrogen gamma rays in the acetone and acetylene.

Silicon is indicated in PINS spectra by gamma rays at 1779 and $3539 \mathrm{keV}$. The $1779-\mathrm{keV}$ inelastic silicon peak is seen in almost all PINS spectra as a background artifact, and hence silicon should always be evaluated using background subtraction. Hydrogen is also a common PINS background element, and the $2223-\mathrm{keV}$ hydrogen peak should always be background-subtracted.

\section{Chlorine Identification}

Chlorine gas, the first chemical warfare agent used in World War I,[3] is now widely used for water purification. [4] As discussed in previous chapters, chlorine produces a rich set of gamma-ray peaks under neutron bombardment, including peaks at 1165, 1763, 1951, and $1959 \mathrm{keV}$.

In PINS assays of pure chlorine gas $\left(\mathrm{Cl}_{2}\right)$, the chlorine inelastic-to-capture peak ratio will be approximately 1.0, and no other chemical elements will be detected, save iron from the steel compressed-gas cylinder.

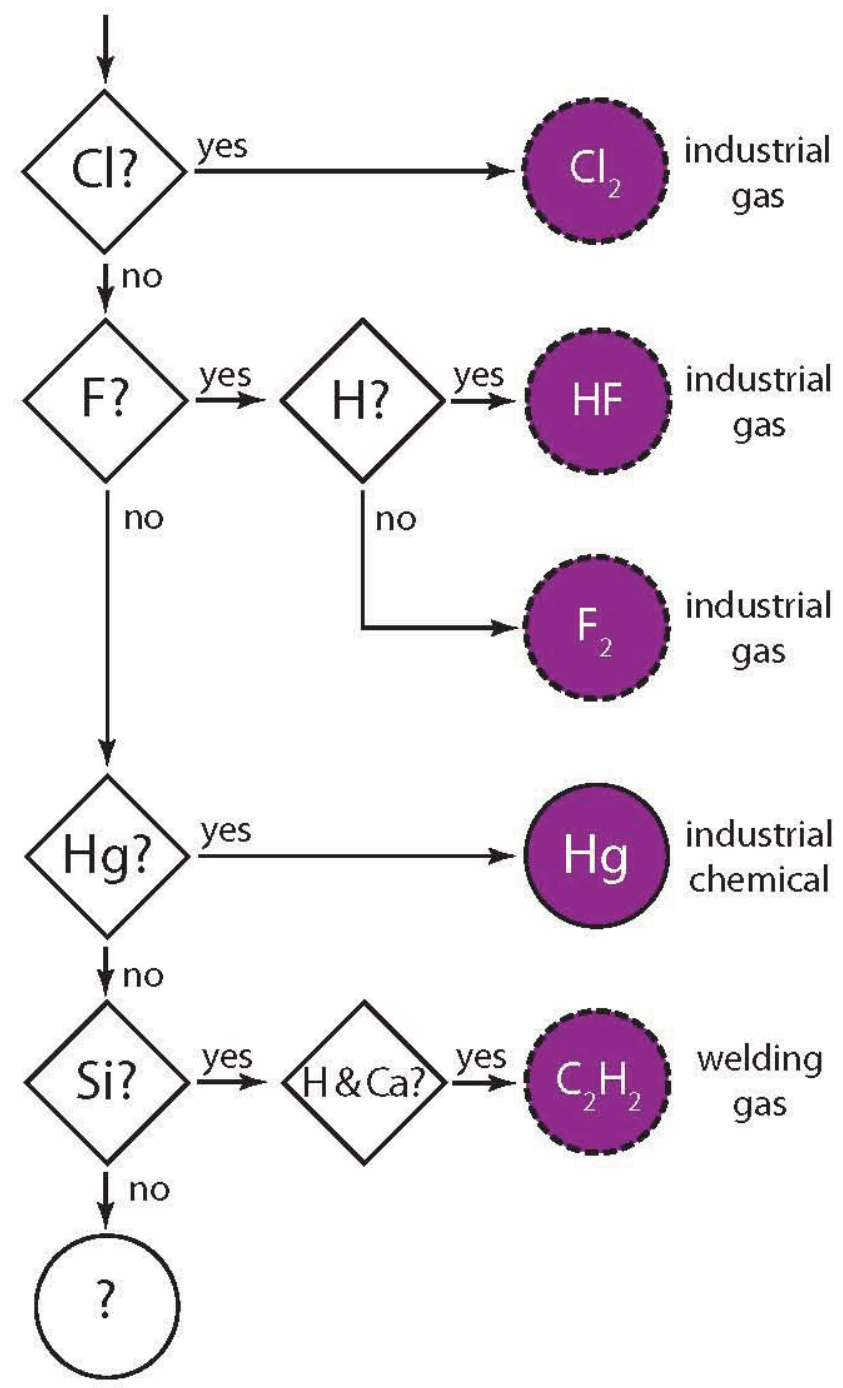

Figure A-1: PINS decision-tree branch for hazardous industrial chemicals.

\section{Hydrogen Fluoride Identification}

Fluorine produces inelastic gamma rays at 197, 1236, 1348, and $1357 \mathrm{keV}$. Alas, the first two gamma ray energies are nearly coincident with a strong germanium peak at 198 $\mathrm{keV}$ and a strong iron peak at $1238 \mathrm{keV}$. Hence fluorine is best identified by from its peak complex at $1348-1357-\mathrm{keV}$ doublet. Absent other PINS key elements, the fluorine compound in the cylinder is gaseous fluorine, $\mathrm{F}_{2}$. [5] If hydrogen is also detected, the cylinder contains hydrogen fluoride, HF.[6] 


\section{Mercury Identification}

Neutrons interact with mercury nuclei to produce gamma rays at $368,439,1694$, and $5966 \mathrm{keV}$. Absent other PINS key element gamma rays in the spectrum, the container holds elemental mercury.[7]

\section{References}

1. W. Braker and A.L. Mossman, Matheson Gas Data Book, Fifth Edition (East Rutherford, NJ: Matheson Gas Products, 1971) page 1.

2. M.J. O'Neil, ed. The Merck Index, 13th Edition (Whitehouse Station, NJ: Merck \& Co., Inc., 2001), article 92.

3. James A.F. Compton, Military Chemical and Biological Agents (Caldwell, NJ: The Telford Press, 1987) page 114. A.M. Prentiss, Chemicals in War (New York: McGraw-Hill, 1937) page 148.

4. C.R. Darnall, "The Purification of Water by Anhydrous Chlorine", American Journal of Public Health, 1 (1911) pages 783-97. A. Wolman and L. H. Enslow, "Chlorine absorption and chlorination of water," Journal of Industrial and Engineering Chemistry, 11 (1911) pages 206-13.

5. The Merck Index, 13th Edition, article 4189. PINS has not been used to assay $\mathrm{F}_{2}$ compressed gas, and hence there is no chemical ID sheet for this chemical. One may expect to observe similar fluorine peaks from $\mathrm{F}_{2}$ gas as have been measured from HF gas, and the hydrogen peak area will be zero after background subtraction.

6. The Merck Index, 13th Edition, article 4817.

7. The Merck Index, 13th Edition, article 5925. 


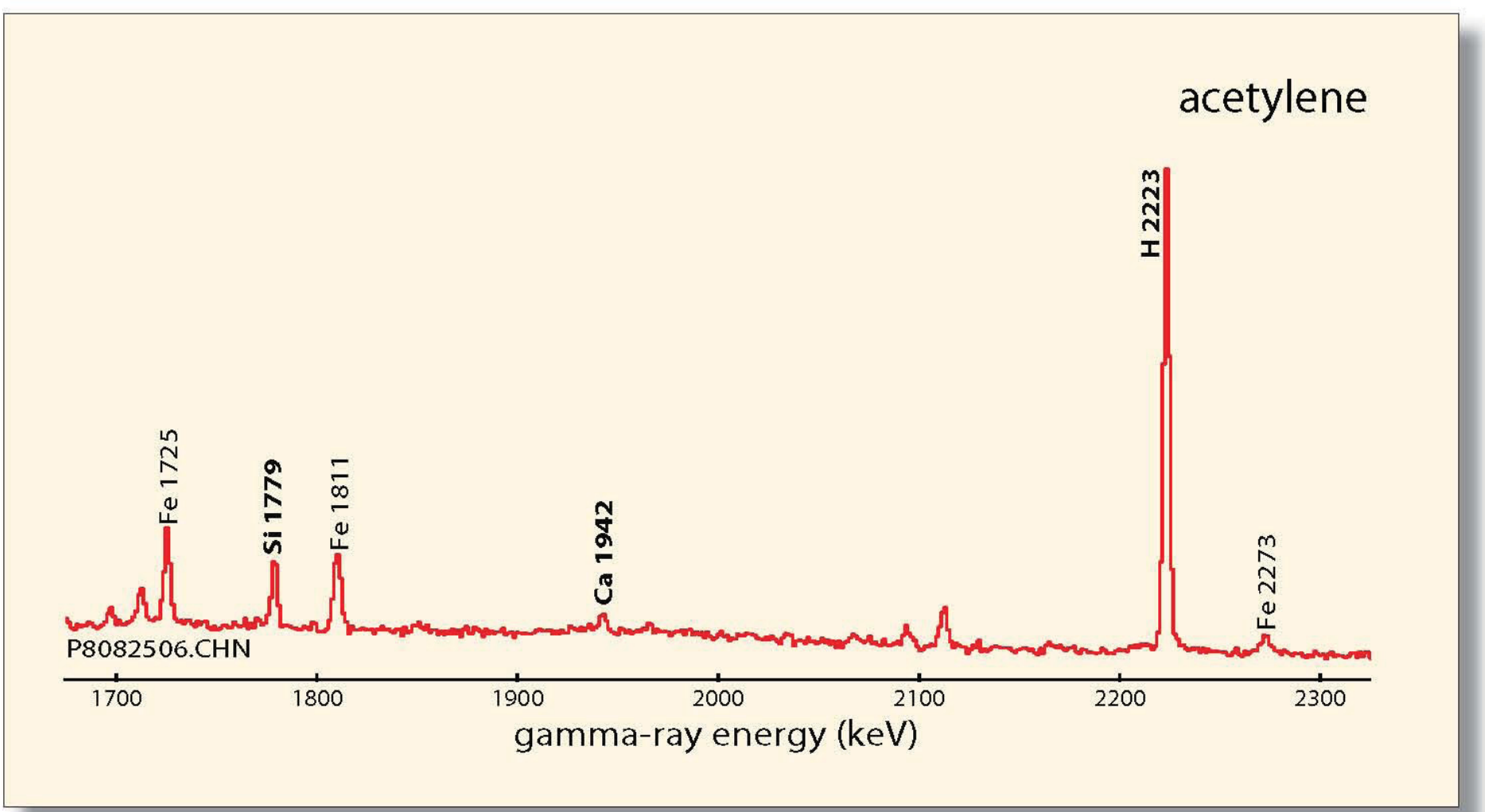

\section{Key spectral features}

- Strong or very strong hydrogen peak at $2223 \mathrm{keV}$

- Moderate or strong calcium peak at $1942 \mathrm{keV}$

- Strong silicon peak at $1779 \mathrm{keV}$

\section{Spectrum ID Tips}

- Acetylene is stored by dissolving it at pressure in acetone; in turn, the acetone is stored in porous ceramic material containing calcium and silicon.

- No other compressed gas is stored in a similar manner.

\section{Caveats}

- Make sure to count and subtract a background spectrum when analyzing a potential acetylene cylinder.

- This spectrum was measured from a compressed-gas cylinder.
Chemical name: acetylene

Chemical formula: $\mathrm{C}_{2} \mathrm{H}_{2}$

Key elements: calcium, silicon, hydrogen

NATO Symbol: $n / a$
Chemical type: welding gas

CWC schedule: $n / a$ 
100 PINS Spectrum Identification Guide 


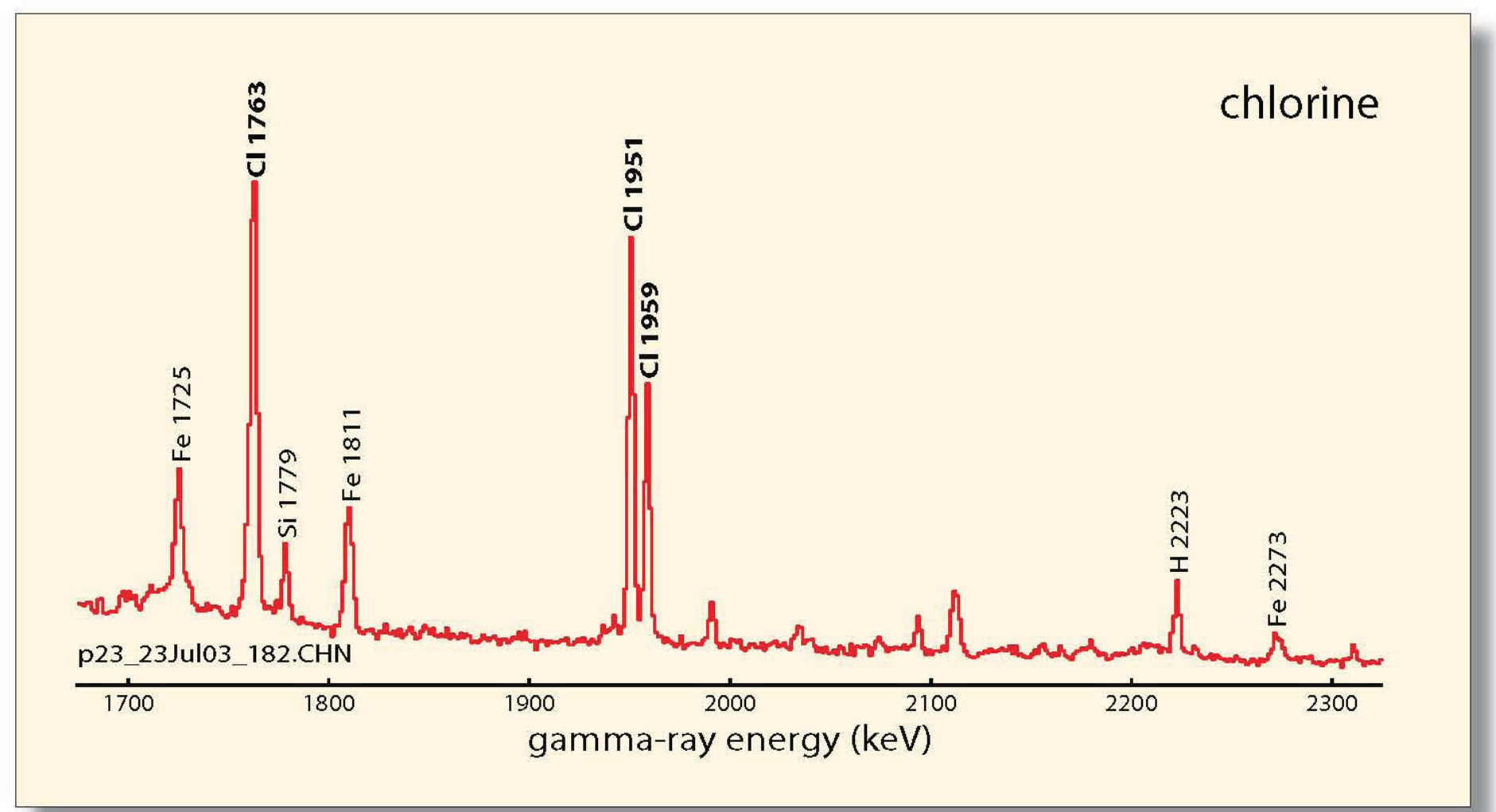

\section{Key spectral features}

- Strong or very strong chlorine peaks at $1165,1763,1951$, and $1959 \mathrm{keV}$

- The chlorine inelastic-to-capture peak ratio, $\mathrm{Cl} 1763 / \mathrm{Cl}$ 1959, will be about 1.0 .

\section{Spectrum ID Tips}

- The hydrogen peak is entirely due to background.

\section{Caveats}

- The PINS phosgene spectrum is nearly identical to the chlorine gas spectrum.

- This spectrum was measured from a compressed-gas cylinder
Chemical name: chlorine

Chemical formula: $\mathrm{Cl}_{2}$

Key elements: chlorine, 100 wt- $\%$

NATO Symbol: $n / a$
Chemical type: industrial gas

CWC schedule: $n / a$ 
102 PINS Spectrum Identification Guide 


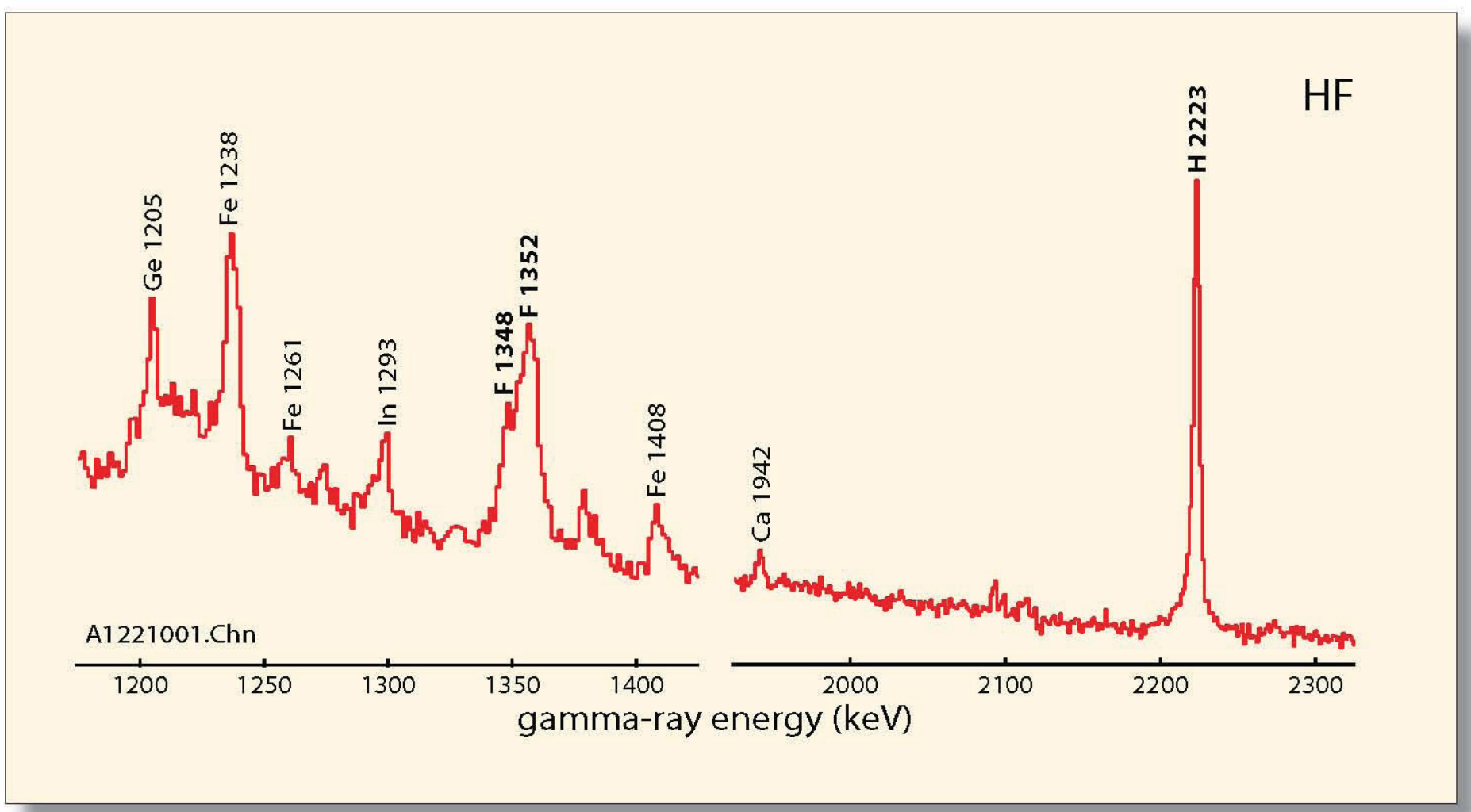

\section{Key spectral features}

- Moderate fluorine peaks at $1348,1352 \mathrm{keV}$

- Moderate to strong hydrogen peak at $2223 \mathrm{keV}$

\section{Spectrum ID Tips}

- The 1348-1352-keV fluorine doublet may not be resolved.

\section{Caveats}

- A hydrogen peak is commonly seen in PINS background spectra. Be sure to measure and subtract a background spectrum when analyzing a potential HF cylinder.

- If the hydrogen peak area after background subtraction is near zero, the likely fill is $\mathrm{F}_{2}$ gas.

- This spectrum was measured from a compressed-gas cylinder.
Chemical name: hydrogen fluoride

Chemical formula: HF

Key elements: fluorine 94.9 wt.- $\%$, hydrogen 5.1 wt.- $\%$
NATO Symbol: $n / a$

Chemical type: industrial chemical

CWC schedule: $n / a$ 
104 PINS Spectrum Identification Guide 


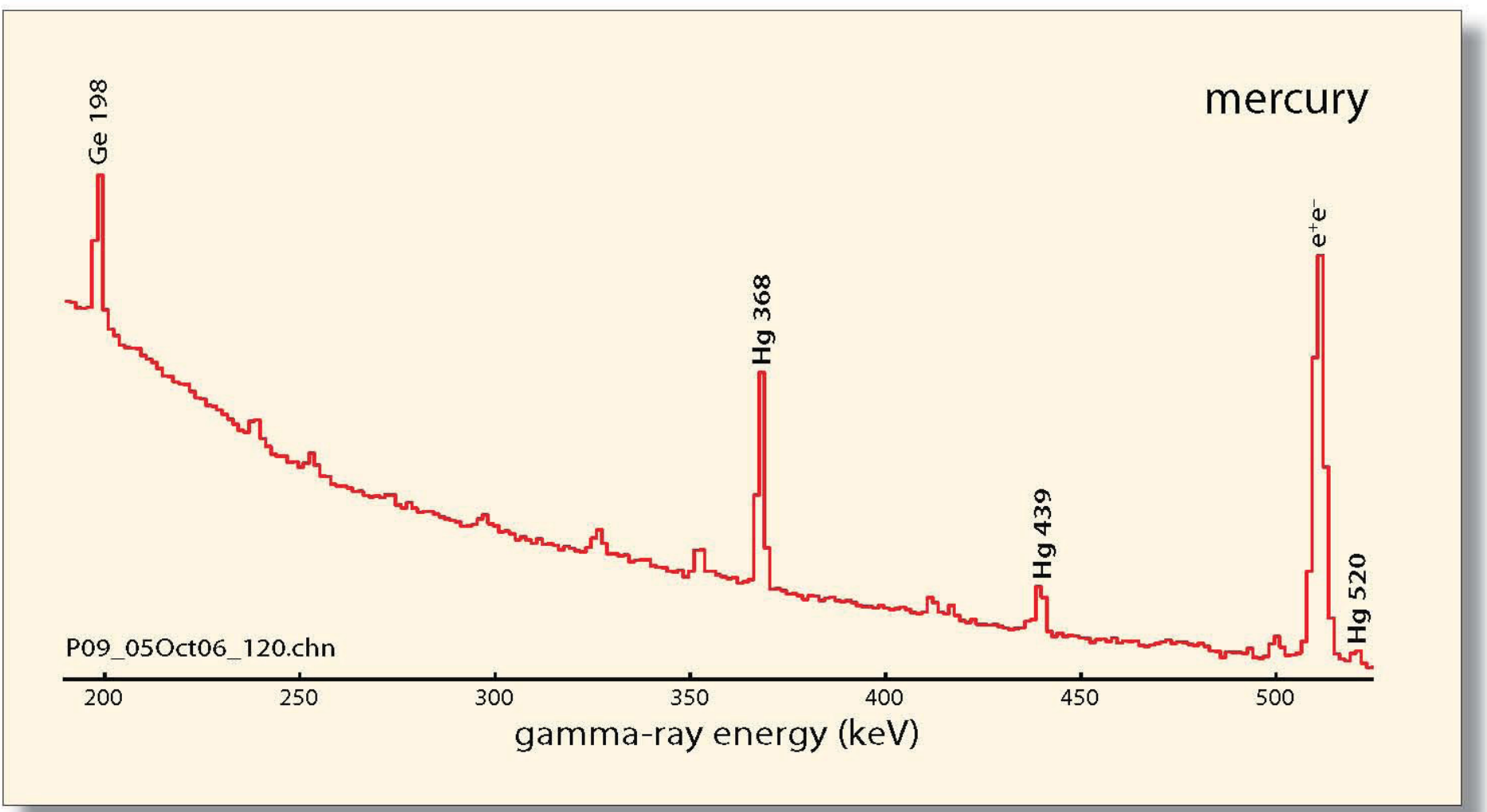

\section{Key spectral features}

- Moderate or strong mercury peaks at 368, 439, 520, 1694, $5966 \mathrm{keV}$

\section{Spectrum ID Tips}

- The hydrogen peak is entirely due to background.

\section{Caveats}

- The 368-keV peak is the most-intense mercury peak.

- This spectrum was measured from a polyethylene reagent bottle containing 240 grams of mercuric oxide.
Chemical name: mercury

Chemical formula: $\mathrm{Hg}$

Key elements: mercury 100 wt- $\%$

NATO Symbol: $n / a$

Chemical type: industrial chemical
CWC schedule: $n / a$

AKA: quicksilver 
106 PINS Spectrum Identification Guide 


\section{Appendix B}

\section{Gamma Ray Energy Table}

\begin{tabular}{|c|c|c|c|c|c|}
\hline Energy & Element & Reaction & $\begin{array}{c}\text { Branching } \\
\text { Ratio* }\end{array}$ & Ref. & Comments \\
\hline 198.4 & germanium** $^{* *}$ & $(\mathrm{n}, \gamma)$ & 100 & 3 & Ge-71 coincidence sum peak \\
\hline 217.3 & bromine & $\left(\mathrm{n}, \mathrm{n}^{\prime} \gamma\right)$ & 47.5 & 2 & \\
\hline 245.7 & bromine & $(\mathrm{n}, \gamma)$ & 11.4 & 4 & \\
\hline 260.9 & bromine & $\left(n, n^{\prime} \gamma\right)$ & 40 & 2 & \\
\hline 264.7 & arsenic & $\left(n, n^{\prime} \gamma\right)$ & 67 & 2 & \\
\hline 275.9 & bromine & $\left(\mathrm{n}, \mathrm{n}^{\prime} \gamma\right)$ & 100 & 2 & \\
\hline 279.5 & arsenic & $\left(\mathrm{n}, \mathrm{n}^{\prime} \gamma\right)$ & 100 & 2 & \\
\hline 326.3 & germanium & $(\mathrm{n}, \gamma)$ & 5.0 & 4 & \\
\hline 352.2 & iron & $(\mathrm{n}, \gamma)$ & 11.7 & 4 & \\
\hline 368.1 & mercury & $(\mathrm{n}, \gamma)$ & 81.3 & 4 & \\
\hline 439.2 & mercury & $\left(n, n^{\prime} \gamma\right)$ & 100 & 2 & \\
\hline 440.0 & sodium & $\left(\mathrm{n}, \mathrm{n}^{\prime} \gamma\right)$ & 100 & 2 & \\
\hline 472.2 & sodium & $(\mathrm{n}, \gamma)$ & 59.8 & 3,4 & \\
\hline 477.6 & boron & ${ }^{10} \mathrm{~B}(\mathrm{n}, \alpha){ }^{7} \mathrm{Li}$ & - & 4 & Doppler-broadened peak \\
\hline 500.2 & germanium & $(\mathrm{n}, \gamma)$ & 3.6 & 4 & \\
\hline 511.0 & $\mathrm{e}+\mathrm{e}-$ & annihilation & 100 & 1 & \\
\hline 516.7 & chlorine & $(\mathrm{n}, \gamma)$ & 18.5 & 4 & \\
\hline 520.3 & mercury & $\left(n, n^{\prime} \gamma\right)$ & 31 & 2 & \\
\hline 537.4 & lead & $\left(\mathrm{n}, \mathrm{n}^{\prime} \gamma\right)$ & 32 & 2 & \\
\hline 562.9 & germanium & $\left(\mathrm{n}, \mathrm{n}^{\prime} \gamma\right)$ & 20 & 2 & \\
\hline 595.9 & germanium & $\left(\mathrm{n}, \mathrm{n}^{\prime} \gamma\right)$ & 100 & 2 & \\
\hline 661.7 & cesium-137 & $\beta$ decay & 85.2 & 1 & \\
\hline 689.6 & germanium & $\left(\mathrm{n}, \mathrm{n}^{\prime} \gamma\right)$ & - & 3 & \\
\hline 788.4 & chlorine & $(\mathrm{n}, \gamma)$ & 15 & 4 & \\
\hline 803.0 & lead & $\left(n, n^{\prime} \gamma\right)$ & 100 & 2 & \\
\hline 846.8 & iron & $\left(\mathrm{n}, \mathrm{n}^{\prime} \gamma\right)$ & 100 & 2 & \\
\hline 896.2 & bismuth & $\left(n, n^{\prime} \gamma\right)$ & 100 & 2 & \\
\hline 935.6 & chromium & $\left(\mathrm{n}, \mathrm{n}^{\prime} \gamma\right)$ & 8.4 & 2 & \\
\hline 983.5 & titanium & $\left(\mathrm{n}, \mathrm{n}^{\prime} \gamma\right)$ & 100 & 2 & \\
\hline 991.6 & zinc & $\left(\mathrm{n}, \mathrm{n}^{\prime} \gamma\right)$ & 100 & 2,3 & \\
\hline 1014.4 & aluminum & $\left(n, n^{\prime} \gamma\right)$ & 100 & 2 & \\
\hline 1039.2 & zinc & $\left(n, n^{\prime} \gamma\right)$ & 57 & 2 & also Ge $1039.5\left(n, n^{\prime} \gamma\right)$ peak, 32\% \\
\hline 1077.4 & zinc & $\left(n, n^{\prime} \gamma\right)$ & 34 & 2 & also $\mathrm{Zn}(\mathrm{n}, \gamma)$ peak, $18.9 \%$ \\
\hline 1097.0 & indium & $(n, \gamma)$ & 11.9 & 4 & \\
\hline 1164.7 & chlorine & $(n, \gamma)$ & 100 & 4 & \\
\hline 1171.3 & tin & $\left(n, n^{\prime} \gamma\right)$ & 100 & 2 & \\
\hline 1173.2 & cobalt -60 & $\beta$ decay & 100 & 1 & also $\mathrm{Ni}\left(\mathrm{n}, \mathrm{n}^{\prime} \gamma\right)$ peak, $13 \%$ \\
\hline
\end{tabular}




\begin{tabular}{|c|c|c|c|c|c|}
\hline Energy & Element & Reaction & $\begin{array}{c}\text { Branching } \\
\text { Ratio* }\end{array}$ & Ref. & Comments \\
\hline 1219.5 & chlorine & $\left(n, n^{\prime} \gamma\right)$ & 77.5 & 2 & \\
\hline 1229.7 & tin & $\left(n, n^{\prime} \gamma\right)$ & 82 & 2 & \\
\hline 1238.3 & iron & $\left(n, n^{\prime} \gamma\right)$ & 10.5 & 2 & \\
\hline 1260.6 & iron & $(n, \gamma)$ & 2.6 & 4 & \\
\hline 1266.1 & phosphorus & $\left(n, n^{\prime} \gamma\right)$ & 100 & 2 & \\
\hline 1267.2 & potassium & $\left(n, n^{\prime} \gamma\right)$ & 52 & 2 & \\
\hline 1293.4 & indium & $(\mathrm{n}, \gamma)$ & 17.6 & 4 & \\
\hline 1332.5 & cobalt- 60 & $\beta$ decay & 100 & 1 & also $\mathrm{Ni}\left(\mathrm{n}, \mathrm{n}^{\prime} \gamma\right)$ peak, $60 \%$ \\
\hline 1348.0 & fluorine & $\left(\mathrm{n}, \mathrm{n}^{\prime} \gamma\right)$ & 45.3 & 2 & \\
\hline 1356.5 & fluorine & $\left(n, n^{\prime} \gamma\right)$ & 100 & 2 & \\
\hline 1381.5 & titanium & $(\mathrm{n}, \gamma)$ & 69.1 & 4 & \\
\hline 1408.2 & iron & $\left(n, n^{\prime} \gamma\right)$ & 3.5 & 2 & \\
\hline 1434.2 & chromium & $\left(n, n^{\prime} \gamma\right)$ & 100 & 2 & \\
\hline 1454.3 & nickel & $\left(n, n^{\prime} \gamma\right)$ & 100 & 2 & \\
\hline 1460.8 & potassium- 40 & $\mathrm{EC}$ & 10.7 & 1 & $\mathrm{EC} \equiv$ electron capture \\
\hline 1609.0 & bismuth & $\left(\mathrm{n}, \mathrm{n}^{\prime} \gamma\right)$ & 66 & 2 & \\
\hline 1612.7 & iron & $(\mathrm{n}, \gamma)$ & 6.0 & 4 & \\
\hline 1634.6 & nitrogen & $\left(\mathrm{n}, \mathrm{n}^{\prime} \gamma\right)$ & 67 & 2 & seen only in DT-excited spectra \\
\hline 1693.9 & mercury & $(\mathrm{n}, \gamma)$ & 14.1 & 2 & \\
\hline 1725.1 & iron & $(\mathrm{n}, \gamma)$ & 8.0 & 2 & \\
\hline 1763.3 & chlorine & $\left(\mathrm{n}, \mathrm{n}^{\prime} \gamma\right)$ & 100 & 2 & \\
\hline 1778.8 & silicon & $\left(\mathrm{n}, \mathrm{n}^{\prime} \gamma\right)$ & 100 & 2 & \\
\hline 1810.5 & iron & $\left(n, n^{\prime} \gamma\right)$ & 6.9 & 2 & \\
\hline 1942.0 & calcium & $(\mathrm{n}, \gamma)$ & 72.5 & 4 & \\
\hline 1950.9 & chlorine & $(\mathrm{n}, \gamma)$ & 21.7 & 4 & \\
\hline 1959.1 & chlorine & $(\mathrm{n}, \gamma)$ & 14.6 & 4 & \\
\hline 2094.6 & iron & $\left(n, n^{\prime} \gamma\right)$ & 1.1 & 2 & \\
\hline 2112.9 & iron & $\left(n, n^{\prime} \gamma\right)$ & 3.2 & 2 & \\
\hline 2211.8 & aluminum & $\left(\mathrm{n}, \mathrm{n}^{\prime} \gamma\right)$ & 52 & 2 & Doppler broadened \\
\hline 2223.3 & hydrogen & $(\mathrm{n}, \gamma)$ & 100 & 4 & \\
\hline 2230.2 & sulfur & $\left(n, n^{\prime} \gamma\right)$ & 100 & 2 & \\
\hline 2233.4 & phosphorus & $\left(n, n^{\prime} \gamma\right)$ & 29 & 2 & \\
\hline 2273.3 & iron & $\left(\mathrm{n}, \mathrm{n}^{\prime} \gamma\right)$ & 2.0 & 2 & \\
\hline 2379.7 & sulfur & $(\mathrm{n}, \gamma)$ & 44.5 & 4 & \\
\hline 2614.5 & thallium-208 & $\beta$ decay & 99.8 & 2,3 & also $\mathrm{Pb}\left(\mathrm{n}, \mathrm{n}^{\prime} \gamma\right)$ peak, $66 \%$ \\
\hline 2721.3 & iron & $(\mathrm{n}, \gamma)$ & 1.7 & 4 & \\
\hline 2813.8 & potassium & $\left(n, n^{\prime} \gamma\right)$ & 100 & 2 & \\
\hline 2863.9 & chlorine & $(\mathrm{n}, \gamma)$ & 6.9 & 4 & \\
\hline 3061.7 & chlorine & $(\mathrm{n}, \gamma)$ & 3.9 & 4 & \\
\hline 3267.4 & iron & $(\mathrm{n}, \gamma)$ & 1.5 & 4 & \\
\hline
\end{tabular}




\begin{tabular}{|c|c|c|c|c|c|}
\hline Energy & Element & Reaction & $\begin{array}{c}\text { Branching } \\
\text { Ratio* }\end{array}$ & Ref. & Comments \\
\hline 3414.2 & iron & $(\mathrm{n}, \gamma)$ & 1.2 & 4 & \\
\hline 3436.6 & iron & $(\mathrm{n}, \gamma)$ & 1.8 & 4 & \\
\hline 3539.1 & silicon & $(n, \gamma)$ & 68.0 & 4 & \\
\hline 3900.3 & phosphorus & $(\mathrm{n}, \gamma)$ & 16.0 & 4 & \\
\hline 4218.3 & iron & $(\mathrm{n}, \gamma)$ & 4.3 & 4 & \\
\hline 4934.4 & silicon & $(n, \gamma)$ & 62.7 & 4 & \\
\hline 5269.2 & nitrogen & $(n, \gamma)$ & 29.7 & 4 & \\
\hline 5409.3 & iron & $(\mathrm{n}, \gamma)$ & - & 4 & Fe 5920 single-escape peak \\
\hline 5420.5 & sulfur & $(\mathrm{n}, \gamma)$ & 59.1 & 4 & \\
\hline 5507.5 & iron & $(n, \gamma)$ & - & 4 & $\mathrm{Fe} 6018$ single-escape peak \\
\hline 5715.3 & chlorine & $(\mathrm{n}, \gamma)$ & 5.5 & 4 & \\
\hline 5920.3 & iron & $(\mathrm{n}, \gamma)$ & 9.0 & 4 & \\
\hline 5966.2 & mercury & $(\mathrm{n}, \gamma)$ & 13.9 & 4 & \\
\hline 6018.5 & iron & $(\mathrm{n}, \gamma)$ & 9.0 & 4 & \\
\hline 6110.9 & chlorine & $(\mathrm{n}, \gamma)$ & 20.0 & 4 & \\
\hline 6129.3 & oxygen & $\left(\mathrm{n}, \mathrm{n}^{\prime} \gamma\right)$ & 100 & 2 & \\
\hline 6419.9 & calcium & $(\mathrm{n}, \gamma)$ & 38 & & \\
\hline 6785.3 & phosphorus & $(\mathrm{n}, \gamma)$ & 13.0 & 4 & \\
\hline 7413.8 & chlorine & $(\mathrm{n}, \gamma)$ & 10.4 & 4 & \\
\hline 7631.1 & iron & $(\mathrm{n}, \gamma)$ & 28.5 & 4 & \\
\hline 7645.5 & iron & $(\mathrm{n}, \gamma)$ & 24.1 & 4 & \\
\hline 7723.9 & aluminum & $(\mathrm{n}, \gamma)$ & 27.4 & 4 & \\
\hline 7790.2 & chlorine & $(\mathrm{n}, \gamma)$ & 8.6 & 4 & \\
\hline 9298.4 & iron & $(\mathrm{n}, \gamma)$ & 4.1 & 4 & \\
\hline 9807.2 & nitrogen & $(\mathrm{n}, \gamma)$ & - & 4 & N 10829 double-escape peak \\
\hline 10196.2 & germanium & $(\mathrm{n}, \gamma)$ & - & 3,4 & Ge-74 coincidence sum peak \\
\hline 10318.2 & nitrogen & $(\mathrm{n}, \gamma)$ & - & & N 10829 single-escape peak \\
\hline 10829.2 & nitrogen & $(n, \gamma)$ & 14.1 & 4 & \\
\hline
\end{tabular}

*The branching ratio is the number of gamma rays of a given energy emitted per 100 decays of a nucleus. For capture and radionuclide gamma rays, the stated branching ratios are absolute. For inelastic scattering gamma rays, the branching ratios have been normalized to the strongest peak.

** The strongest capture and inelastic gamma rays for each element are denoted in bold type. 


\section{References}

1. K. Debertin and R.G. Helmer, Gamma- and X-Ray Spectrometry with Semiconductor Detectors (Amsterdam: Elsevier Science, 1988) pages 351-367.

2. A.M. Demidov et al., Atlas of Gamma-Ray Spectra from the Inelastic Scattering of Reactor Fast Neutrons (Moscow: Atomizdat, 1978).

3. R.B. Firestone, ed., Table of Isotopes, Eighth Edition (New York: John Wiley \& Sons, 1996)

4. M.A. Lone, R.A. Leavitt, and D.A. Harrison, "Prompt Gamma Rays from Thermal-Neutron Capture”, Atomic Data and Nuclear Data Tables, 26, (1981) pages 511-559. 


\section{Index}

Acetylene, 97, 99

Agent precursor, See Arsenic trichloride

Antifreeze, 83-85

Arsenicals, 39-50

arsenic trichloride, 39, 43-44

arsine, 39, 41-42

blue ring-2, agent, $39,45-46$

lewisite, 39, 47-48

winter mustard, 39, 49-50

Arsenic trichloride, 39, 43-44

Arsenic trihydride, See Arsine

Arsine, 39, 41-42

Atkins, P.W., 84

Balo, P.A., 9

$\mathrm{BBC}$, See CA riot agent

Beal, R.E., 84

Bis (2-chloroethyl) sulfide, See Mustard agent

Bleach, decontamination, 83, 91-94

calcium, 91-92

sodium, 93-94

Blister agents

blue ring-2, 39, 45-46

lewisite, 39, 47-48

mustard, 22, 39, 67-68

nitrogen mustard-3, 71-72

winter mustard, 39, 49-50

Blue Ring-2, agent, 39, 45-46

Boron, 5, 20, 85

BR-2, See Blue Ring-2

Braker, W., 98

Branching ratio, 109

Bromine, 73

Bromine-based chemical, 73-75

Bromobenzylcyanide, See CA riot agent

C-4 plastic explosive, 77

CA riot agent, 73, 75

Cadmium, 5

Calcium decontamination bleach, 91-92

Calcium hypochlorite, See Calcium decontamination bleach

Calcium sulfate dehydrate, See Plaster-of-Paris

Californium-252, 11

Camite, See CA riot agent

Carbonyl chloride, See Phosgene
CF-252, 12,

CG, See Phosgene

Chlorine (gas), 101

Chlorine-based chemicals, 51-72, 101

CG, 21, 53-54

chlorine gas, 101

CK, 21, 52, 69-70

$\mathrm{CNB}, 52,55-56$

CNS, $52,54,57-58$

FM, 51, 61-62

FS, 51, 65-66

$\mathrm{HC}, 51,63-64$

KJ, 51, 59-60

mustard agent, 67-68

nitrogen mustard-3, 71-72

Chlorosulfonic acid, 51, 65-66

Clorox, See sodium decontamination bleach

Chrien, R.E., 9

CK blood agent, 69-70

$\mathrm{Cl} \mathrm{i} / \mathrm{c}$ ratio, $39,40,52$

CNB riot agent, 51-52, 55-56

CNS riot agent, 57-58

Compton, J.A.F., 30, 40, 52, 73, 98

Composition B high explosive, 77

Concrete, 87-88

Cyanogen chloride, See CK blood agent

DA-PD blister agent, See Blue Ring-2

Darnall, C.R., 98

DD fusion reaction, 2

Debertin, K., 23, 110

Decision tree, arsenical chemicals branch, 39

bromine-based chemical branch, 73

chlorine-based chemicals branch, 51

industrial chemicals, 97

methods, 21-22, 25-26

nitrogen-based chemicals branch, 77

phosphorus-based chemicals branch, 29

practice-fill chemicals, 83

Delayed neutron activation analysis, 1

Demidov, A.M., 9, 23, 110

Dichloro (2-chlorovinyl) arsine, See Lewisite

Divadeenam, M., 84 
Double-escape event, 8

DT fusion reaction, 2

Eisenhauer, C.M., 9

Emsley, J., 40, 84

Energies, gamma-ray, 107-110

Enslow, L.H., 98

Erdtmann, G., 23

Ethyl N,N-dimethylphosphoroamidocyanidate, See GA

Explosives identification, 77-81

$\mathrm{F}_{2}$, See Fluorine gas

Firestone, R.B., 110

Fluorine gas, 97, 103

FM smoke, 51, 61-62

Forrest, L., 30

FS smoke, 22, 51, 65-66

Full energy event, 8

GA nerve agent, 37-38

Gadolinium, 5

Gamma-ray

detector, 12

energy table, 107-110

interactions

Compton scattering, 7

pair production, 7-8

photoelectric, 6

spectra, See individual spectrum ID sheets

spectrum analysis, 17-24

GB nerve agent, 33-34

Gilmore, G., 23

Greenwood, R.C., 9

Grundl, J., 9

H, See Mustard agent

H-PD, See Winter mustard agent

Harris, R., 23

Harrison, D.A., 9, 23, 110

Hazardous industrial chemicals, 97-105

acetylene, 99

chlorine, 101

hydrogen fluoride, 103

mercury, 105

HC smoke, 21, 51, 63-64

HD, See mustard agent

Helmer, R.G., 23, 110

HF, See Hydrogen fluoride

$\mathrm{Hg}$, See mercury

High explosives, 77-81

Hill, C., 23
HN-3, See Nitrogen mustard-3

Holden, N.E., 84

Holte, L., 40

HS, See Mustard agent

HT, See Mustard agent

HTH bleach, See Calcium decontamination bleach

Hydrogen, 73

Hydrogen fluoride gas, 103

Identification logic, spectrum, 25-28

See also Decision tree

Incendiary chemical, See WP

Industrial chemicals, See Hazardous industrial chemicals

Isopropyl methylphosphonofluoridate, See GB

Jeffrey, A. 23

Jordan, C.B., 84

KJ smoke, 51, 59-60

Knauer, J.B., 9

Knoll, Glenn F, 9, 80

Köhler, J., 80

L, See Lewisite

Leavitt, R.A., 9, 23, 110

Lederman, L., 23

Lewisite, 47-48

Lone, M.A., 9, 23, 110

Löst, See mustard agent

Martin, R.C., 9

Mercury, 105

Meyer, R., 80

Molnar, G.L., 9, 23

Mossman, A.L., 98

Mughabghab, S.F., 84

Mustard agent, 22, 51, 67-68

Mustard gas, See Mustard agent

Nerve gas, See GA, GB, VX nerve agents

Neutron activation analysis

delayed, 1

prompt gamma ray, 1-9

Neutron cross sections, 4

Neutron generators, 2

Neutron interactions

elastic scattering, 2-3

inelastic scattering 3

neutron capture, 3-5

Nitrogen, 23, 73

based chemicals, 77-81

gamma-ray peak analysis, 78-80

in explosives, 81 
Nitrogen mustard, 51

Nitrogen mustard-3, 71-72

Nuclear energy levels, 17

O-ethyl-S-(2-diisopropylaminoethyl) methyl phosphonothiolate, See VX

O’Neil, M.J., 40, 80, 84, 98

Operational issues

background gamma rays, 14

control screen, 13

energy resolution, 15-16

Peak

area, 19

detection, 19, 78-80

energy, 18-19

fitting, 18-19

summing, 18-19

PETN high explosive, 77

PGAA, See Neutron activation analysis

PGNAA, See Neutron activation analysis

Phosgene, 53-54

Phosphorus-based chemicals, 29-38

GA, 37-38

GB, 21, 33-34

VX, 21, 35-36

WP, 21, 31-32

PINS equipment

counting geometry, 12

HPGe detector, 12

moderator/shadow-shield assembly, 12

multichannel analyzer, 12

neutron source, Cf-252, 1-2, 11

notebook computer, 12-13

PINS software

data acquisition, 13

data analysis, $13,18-22,77-80$

Plaster-of-Paris, 89-90

Polyisobutylene, 77

POP, See Plaster-of-Paris

Portland concrete, See Concrete

Practice-fill chemicals, 83-96

calcium decontamination bleach, 91-92

concrete, 87-88

plaster-of-Paris, 89-90

sand, 95-96

sodium decontamination bleach, 93-94

water/antifreeze, $85-86$

Prentiss, A.M., 52, 73, 98
Prompt neutron activation analysis, 1

Pulse pile-up, 16

Quicksilver, See mercury

Radioisotopic neutron sources, 1-2

RDX high explosive, 77

Reaction cross section, 4

Riot control agents

$\mathrm{CA}, 73,75$

CNB, $51,55-56$

CNS, 51, 57-58

Ross, S.M., 80

SA, See Arsine

Sand, 95-96

Sarin, See GB nerve agent

Siedenstrang, A.L., 16

Silica, See sand

Silicon dioxide, See sand

Single-escape event, 7

Sodium decontamination bleach, 93-94

Sodium hypochlorite, See Sodium decontamination bleach

Soyka, W., 23

Spectrum analysis

decision tree, 21, 25

detection confidence, $20,78-80$

fundamentals, 17-20, 25-28

identification sheet description, 27-28

See also Spectrum identification sheets

Spectrum identification sheets

bleach, decontamination

calcium, 91-

sodium, 93-

blue ring-2, agent, 39, 45-46

CA, 73, 75

CG, 53-54, 57

chlorine gas, 101

CK, 51, 69-70

CNB, $51,55-56$

CNS, 51, 57-58

concrete, $87-88$

FM, 51, 61-62

FS, 51, 65-66

GA, 37-38

GB, 33-39

$\mathrm{HC}, 51,63-64$

high explosives, 81

hydrogen fluoride gas, 103

lewisite, 45-46 
mercury, 105

mustard, 67-68

nitrogen mustard-3, 71-72

plaster-of-Paris, 89-90

sand, 95-96

VX, 35-36

water/antifreeze, 83,85

winter mustard, 39, 49-50

WP, 31-32

Stannic chloride, See KJ

Sulfur mustard, See Mustard agent

Summing, 19

$\mathrm{t}^{*}, 78-80$

Tabun, See GA nerve agent

Taguchi, K., 16

Taylor, J.R., 23, 80

Thermal neutron capture cross sections of the elements, 5

Tin tetrachloride, See KJ

Titanium tetrachloride, See FM

TNT, 77

Tucker, J.B., 30

U.S. Army Field Manual 3-9 (1975), 52, 73

U.S. Army Field Manual 3-11.9, 30, 40, 52, 73

VX nerve agent, 35-36

Water-antifreeze mixture, 85

White phosphorus smoke, 31-32

Winter mustard agent, 39, 49-50

Winterlöst, See Winter mustard agent

WL, See Winter mustard agent

Wolman, A., 98

WP, See White phosphorus

Yperite, See Mustard agent

Zellerman, R.A., 40 

